University of Tennessee Health Science Center

UTHSC Digital Commons

$12-2010$

\title{
Effect of Fixation Using Locked Compression Plate versus Lag Screws on Biomechanics of Talonavicular Joint: A Human Cadaveric Foot Model
}

Rahul Sudheer Ghotge

University of Tennessee Health Science Center

Follow this and additional works at: https://dc.uthsc.edu/dissertations

Part of the Medical Sciences Commons, and the Therapeutics Commons

\section{Recommended Citation}

Ghotge, Rahul Sudheer , "Effect of Fixation Using Locked Compression Plate versus Lag Screws on Biomechanics of Talonavicular Joint: A Human Cadaveric Foot Model" (2010). Theses and Dissertations (ETD). Paper 88. http://dx.doi.org/10.21007/etd.cghs.2010.0109.

This Thesis is brought to you for free and open access by the College of Graduate Health Sciences at UTHSC Digital Commons. It has been accepted for inclusion in Theses and Dissertations (ETD) by an authorized administrator of UTHSC Digital Commons. For more information, please contact jwelch30@uthsc.edu. 


\title{
Effect of Fixation Using Locked Compression Plate versus Lag Screws on Biomechanics of Talonavicular Joint: A Human Cadaveric Foot Model
}

\begin{abstract}
Introduction: Talonavicular fusion is a surgery used for treating many hind foot pathologies. A problem associated with the procedure is non-union which may be due to inadequate stabilization. The objective of our study was to compare the effect of two surgical fixation techniques on the motion and biomechanics of the talonavicular joint in a human cadaveric foot model.

Materials and Methods: Thirteen human cadaveric foot specimens were prepared, mounted in a multiaxis programmable robot, and loaded using four loading scenarios. Each of the four loading scenarios consisted of a constant Achilles tendon load of $350 \mathrm{~N}$ followed by either internal or external rotation (moment limit 10Nm) and a compressive load (load limit 850N) applied to the specimens either sequentially after rotation or simultaneously with the rotation. Each specimen was thus subjected to four tests - internal rotation sequential test, internal rotation simultaneous test, external rotation sequential test and external rotation simultaneous test. Each foot specimen was tested in intact state and retested after fixation using either a locked compression plate plus one screw or two screws. Six specimens (plate screw group) received the plate plus a lag screw, where the screw was inserted in a retrograde manner. Seven specimens (two screw group) received two lag screws, both inserted in retrograde manner. Three dimensional targets were fixed to the talus and navicular bones and the motion of the targets was tracked using an optoelectrical camera. The motion was analyzed at a point located in the talonavicular joint space. The relative translational and rotational motions between the two bones were analyzed in two different coordinate frames: one aligned with the anatomical planes (sagittal, coronal and transverse planes) of the body (camera frame) and another aligned with the talonavicular joint (joint frame). The relative translations were compared statistically using three factor ANOVA with a mixed model.
\end{abstract}

Results: In the joint frame, talonavicular motion for the plate screw group relative to intact state showed significantly reduced translation along the long axis of the talus during internal rotation sequential test $(0.9 \mathrm{~mm}$ versus $1.8 \mathrm{~mm})$, internal rotation simultaneous test $(1.4 \mathrm{~mm}$ versus $2.0 \mathrm{~mm})$, and external rotation simultaneous test $(0.9 \mathrm{~mm}$ versus $1.9 \mathrm{~mm})$. When the translations were analyzed in the camera frame, no statistically significant differences were observed. When the relative rotations were analyzed in the joint frame, the plate screw group showed significantly reduced flexion-extension motion as compared to the two screws group during the external rotation sequential test (0.6degrees versus 2.5 degrees) and the external rotation simultaneous test (1.0degrees versus 3.0degrees). When the same rotations were analyzed in the camera frame, the plate screw group showed highly significant restriction of flexion extension the external rotation sequential test (0.4degrees versus 2.5 degrees) and the external rotation simultaneous test (0.7degrees versus 3.0degrees).

Conclusion: A human cadaveric foot model was developed to study talonavicular joint biomechanics. The study provided insight into the motion and mechanics of the talonavicular joint and investigated the capability of different surgical fixation techniques to immobilize the talonavicular joint. Compared to two screws, a plate and screw was more effective at preventing separation between the bones along the long axis of the talus, as well as preventing flexion-extension at the talonavicular joint, which should lead to a higher rate of talonavicular joint fusion and reduce the incidence of non-union. The method of transforming the talonavicular motion into its joint coordinate frame (as opposed to an anatomical coordinate frame) highlighted the ability of the plate and screw to prevent joint motion. The method developed in the study provided a better understanding of the local joint biomechanics and can be used to study other joints as well as joint fixation or instrumentation techniques. 


\section{Document Type}

Thesis

\section{Degree Name}

Master of Science (MS)

\section{Program}

Biomedical Engineering and Imaging

\section{Research Advisor}

Denis J. Diangelo, Ph.D.

\section{Keywords}

Arthrodesis, Biomechanics, Bone plate, Bone screws, Coordinate frame Talonavicular

\section{Subject Categories}

Analytical, Diagnostic and Therapeutic Techniques and Equipment | Medical Sciences | Medicine and Health Sciences | Therapeutics 


\title{
EFFECT OF FIXATION USING LOCKED COMPRESSION PLATE VERSUS LAG SCREWS ON BIOMECHANICS OF TALONAVICULAR JOINT: A HUMAN CADAVERIC FOOT MODEL
}

\author{
A Thesis \\ Presented for \\ The Graduate Studies Council \\ The University of Tennessee \\ Health Science Center \\ In Partial Fulfillment \\ Of the Requirements for the Degree \\ Master of Science \\ In the Joint Graduate Program in Biomedical Engineering and Imaging \\ From The University of Tennessee \\ and \\ The University of Memphis
}

By

Rahul Sudheer Ghotge

December 2010 
Copyright (C) 2010 by Rahul Sudheer Ghotge

All rights reserved 


\section{ACKNOWLEDGEMENTS}

I would like to thank all people who were involved in this project and my graduate education at University of Tennessee Health Science Center. Firstly, I thank my advisor Denis DiAngelo, Ph.D. for all his guidance and input. I would like to thank Brian Kelly, Ph.D., who helped me to learn to solve the problems during this project. I would like to thank all the other members of my committee - Gladius Lewis, Ph.D., John Williams, Ph.D., and Richard Kasser, Ph.D. for their recommendations and feedback regarding my research.

I would also like to thank Jaymes Granata, M.D. and Gregory Charles Berlet, M.D. for their help and contribution towards the clinical aspects of this research. I would like to specially thank Elizabeth Tolley, Ph.D. for her help with the statistical analysis of this research project. I appreciate the help and support of Department of Comparative Medicine, UTHSC for use of their radiographic imaging equipment. I also thank the University of Tennessee Health Sciences Library, Randall J. Nelson, Ph.D., and Department of Anatomy and Neurobiology, UTHSC for their support and resources including the set of bones which were photographed for creating the illustrations in this thesis.

I would also like to thank all the students of the Joint and Implant Biomechanics Lab - Yuan Li, Ph.D., Daniel Wido, B.S., Tom Stewart, B.S., Braham Dhillon, M.S., Jie Gao, M.S., Karen Sedacki, M.S. (candidate), Jessica Stubbs, B.S., and Kyle Fraysur, M.S. I also thank Wright Medical Technologies Inc. for their financial and technical support during this research project. Finally, I thank my parents Mr. Sudheer Ghotge, B.E. and Mrs. Veena Ghotge, M.A. for their continued support and guidance throughout my life. 


\begin{abstract}
Introduction: Talonavicular fusion is a surgery used for treating many hind foot pathologies. A problem associated with the procedure is non-union which may be due to inadequate stabilization. The objective of our study was to compare the effect of two surgical fixation techniques on the motion and biomechanics of the talonavicular joint in a human cadaveric foot model.
\end{abstract}

Materials and Methods: Thirteen human cadaveric foot specimens were prepared, mounted in a multi-axis programmable robot, and loaded using four loading scenarios. Each of the four loading scenarios consisted of a constant Achilles tendon load of 350N followed by either internal or external rotation (moment limit $10 \mathrm{Nm}$ ) and a compressive load (load limit $850 \mathrm{~N}$ ) applied to the specimens either sequentially after rotation or simultaneously with the rotation. Each specimen was thus subjected to four tests internal rotation sequential test, internal rotation simultaneous test, external rotation sequential test and external rotation simultaneous test. Each foot specimen was tested in intact state and retested after fixation using either a locked compression plate plus one screw, or two screws. Six specimens (plate screw group) received the plate plus a lag screw, where the screw was inserted in a retrograde manner. Seven specimens (two screws group) received two lag screws, both inserted in retrograde manner. Three dimensional targets were fixed to the talus and navicular bones and the motion of the targets was tracked using an optoelectrical camera. The motion was analyzed at a point located in the talonavicular joint space. The relative translational and rotational motions between the two bones were analyzed in two different coordinate frames: one aligned with the anatomical planes (sagittal, coronal and transverse planes) of the body (camera frame) and another aligned with the talonavicular joint (joint frame). The relative translations were compared statistically using three factor ANOVA with a mixed model.

Results: In the joint frame, talonavicular motion for the plate screw group relative to intact state showed significantly reduced translation along the long axis of the talus during internal rotation sequential test $(0.9 \mathrm{~mm}$ versus $1.8 \mathrm{~mm})$, internal rotation simultaneous test $(1.4 \mathrm{~mm}$ versus $2.0 \mathrm{~mm})$, and external rotation simultaneous test $(0.9 \mathrm{~mm}$ versus $1.9 \mathrm{~mm}$ ). When the translations were analyzed in the camera frame, no statistically significant differences were observed. When the relative rotations were analyzed in the joint frame, the plate screw group showed significantly reduced flexion-extension motion as compared to the two screws group during the external rotation sequential test (0.6degrees versus 2.5 degrees) and the external rotation simultaneous test (1.0degrees versus 3.0degrees). When the same rotations were analyzed in the camera frame, the plate screw group showed highly significant restriction of flexion extension the external rotation sequential test ( 0.4 degrees versus 2.5 degrees) and the external rotation simultaneous test (0.7degrees versus 3.0degrees).

Conclusion: A human cadaveric foot model was developed to study talonavicular joint biomechanics. The study provided insight into the motion and mechanics of the talonavicular joint and investigated the capability of different surgical fixation techniques 
to immobilize the talonavicular joint. Compared to two screws, a plate and screw was more effective at preventing separation between the bones along the long axis of the talus, as well as preventing flexion-extension at the talonavicular joint, which should lead to a higher rate of talonavicular joint fusion and reduce the incidence of non-union. The method of transforming the talonavicular motion into its joint coordinate frame (as opposed to an anatomical coordinate frame) highlighted the ability of the plate and screw to prevent joint motion. The method developed in the study provided a better understanding of the local joint biomechanics and can be used to study other joints as well as joint fixation or instrumentation techniques. 


\section{TABLE OF CONTENTS}

CHAPTER 1: INTRODUCTION ................................................................................ 1

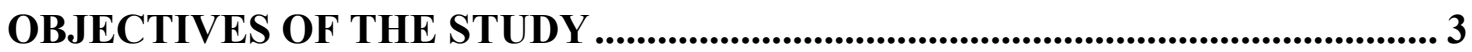

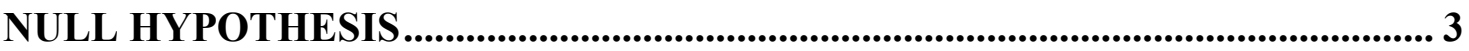

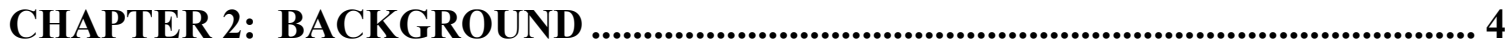

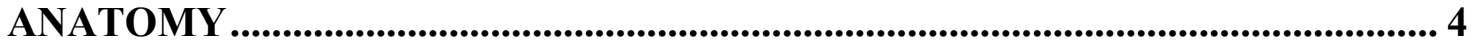

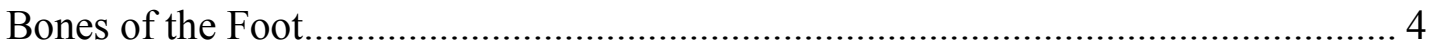

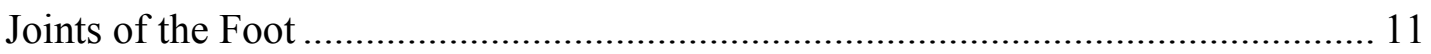

Muscles, Tendons, Ligaments, Nerves, and Blood Vessels of the Foot .................. 16

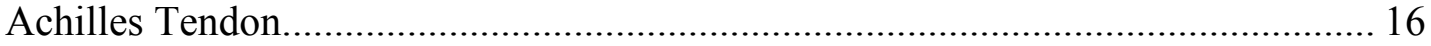

ARCHES OF THE FOOT AND WEIGHT DISTRIBUTION ............................. 18

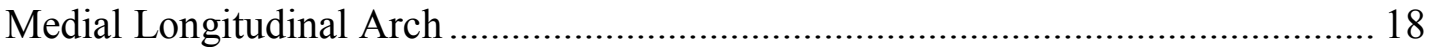

Lateral Longitudinal Arch ............................................................................ 18

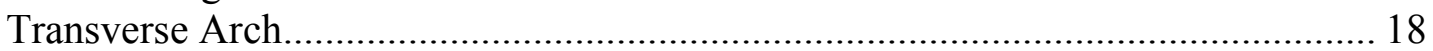

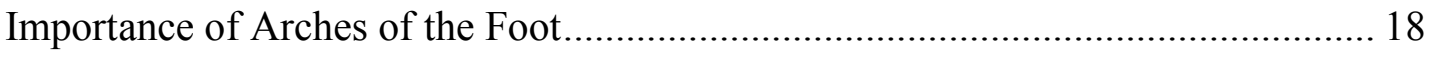

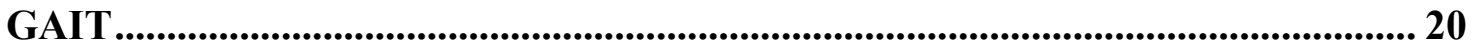

CHAPTER 3: PATHOLOGY AND TREATMENT ........................................... 23

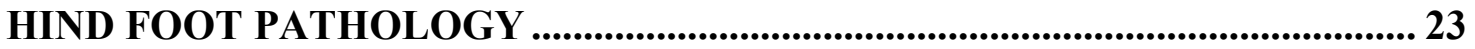

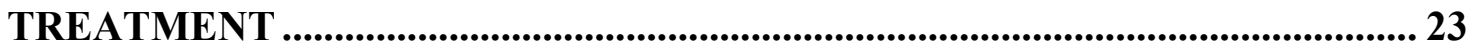

CHAPTER 4: MATERIALS AND METHODS ................................................ 26

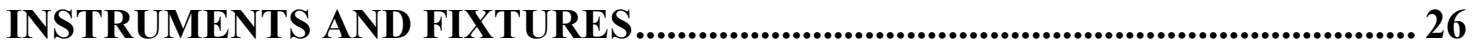

Multiaxis Programmable Test Frame (Spine Robot) ............................................. 26

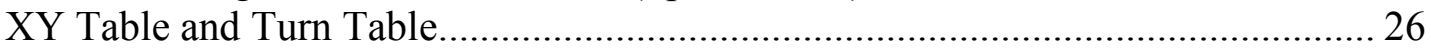

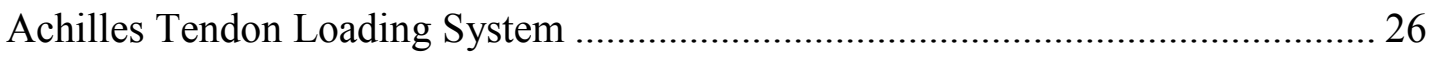

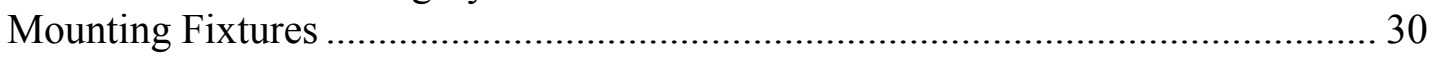

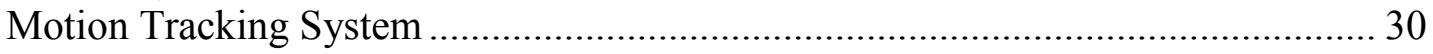

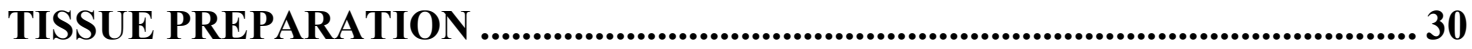

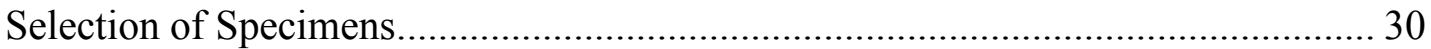

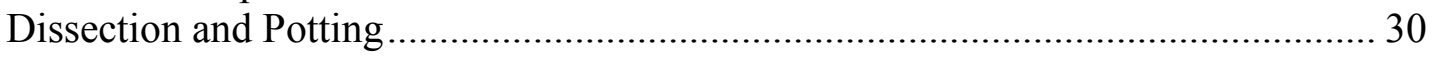

Achilles Tendon Dissection and K-Wire Insertion for Targets ............................. 32

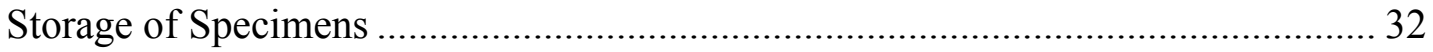

SURGICAL PROCEDURE ..................................................................................... 35

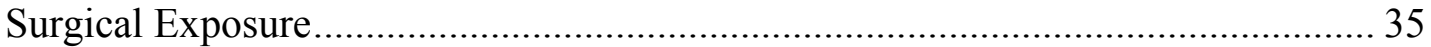

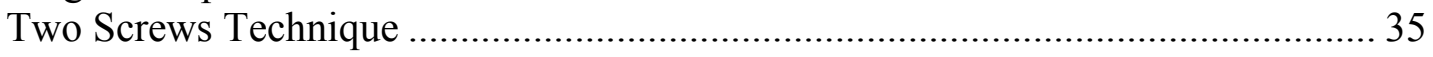

Locked Plate Plus One Screw Technique ......................................................... 35

EXPERIMENTAL METHODOLOGY .................................................................. 40 


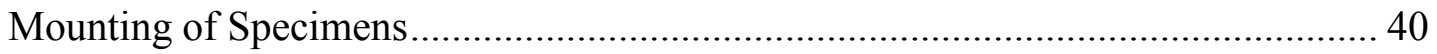

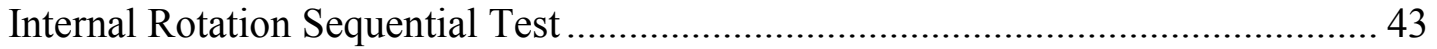

Internal Rotation Simultaneous Test .................................................................. 43

External Rotation Sequential Test ......................................................................... 43

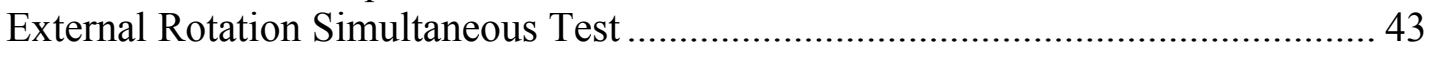

DATA RECORDING AND DATA PROCESSING ..................................................... 46

DATA MANAGEMENT AND STATISTICS ................................................50

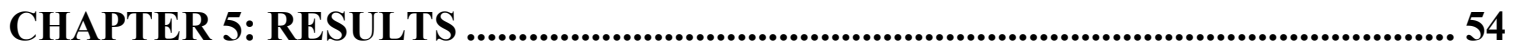

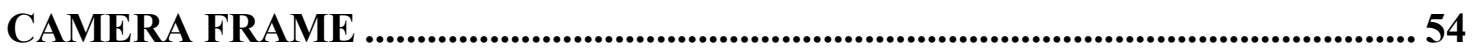

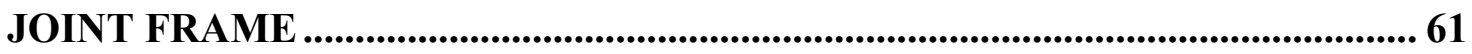

CHAPTER 6: DISCUSSION AND CONCLUSIONS .................................................. 69

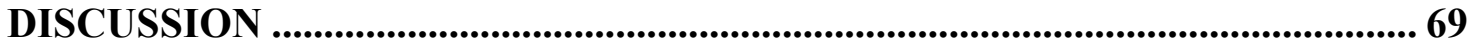

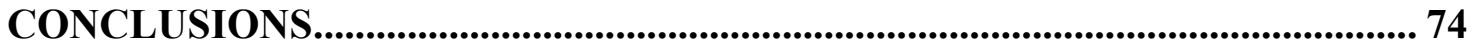

CHAPTER 7: RECOMMENDATIONS FOR FUTURE WORK................................ 75

LIST OF REFERENCES...................................................................................... 77

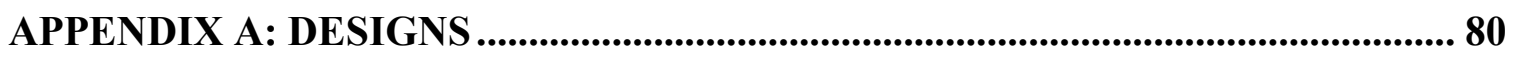

ACHILLES TENDON LOADING SYSTEM...................................................... 80

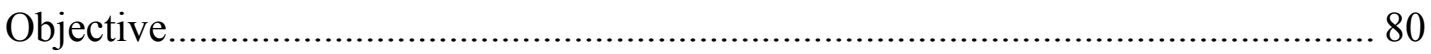

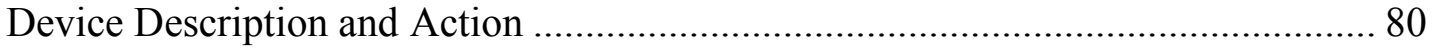

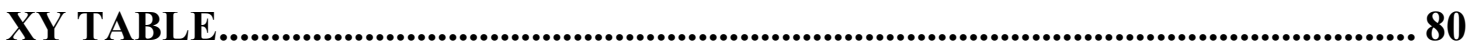

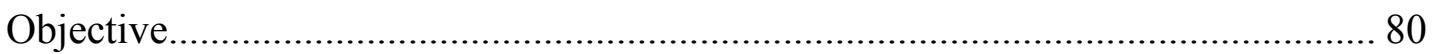

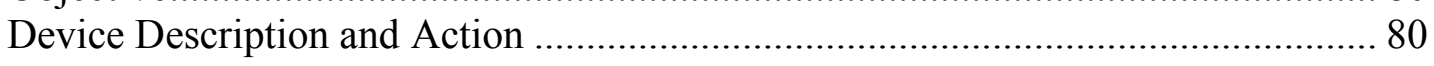

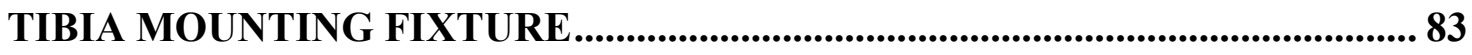

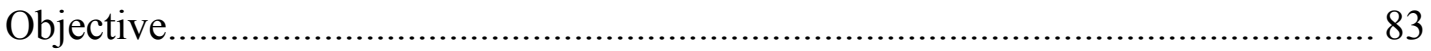

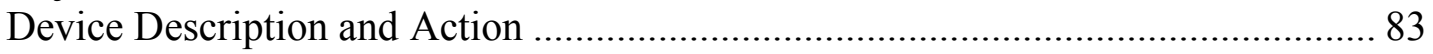

APPENDIX B: STEP-BY-STEP DESCRIPTION OF EXPERIMENT ...................... 86

PREPARATION AND MOUNTING ...................................................................... 86

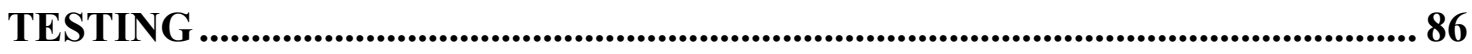

Internal Rotation Sequential Test ..................................................................... 86

Internal Rotation Simultaneous Test …………………......................................... 91

External Rotation Sequential Test..................................................................... 91

External Rotation Simultaneous Test ................................................................... 91

APPENDIX C: MEASUREMENTS OF OFFSETS ...................................................... 93

LOCALIZATION OF POINT J .................................................................................. 93

EFFECT OF SURGERY ON LOCATION OF POINT J ....................................... 93

MEASUREMENT OF OFFSET DISTANCES ........................................................ 93 
Measurement of Offset Distances Using Calipers................................................ 93

Radiographic Measurement of Offset Distances ................................................ 97

APPENDIX D: DERIVATION OF TRANSFORMATION MATRICES ............. 103

APPENDIX E: DATA PROCESSING ............................................................... 107

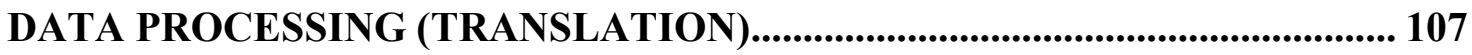

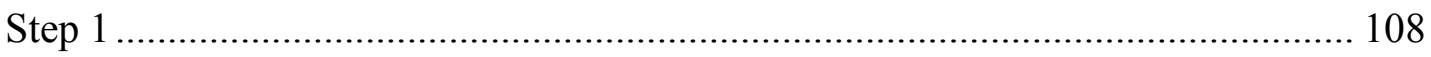

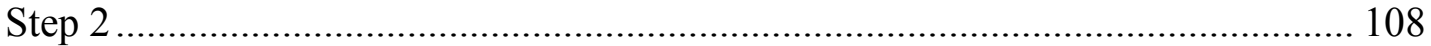

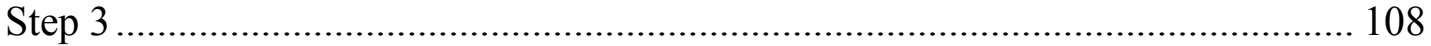

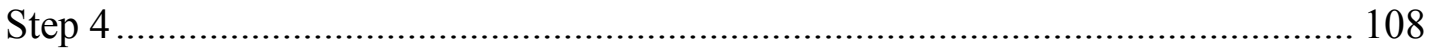

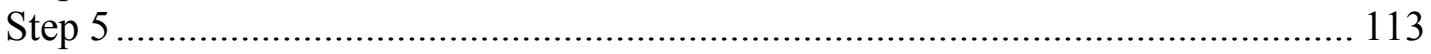

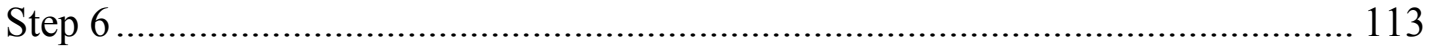

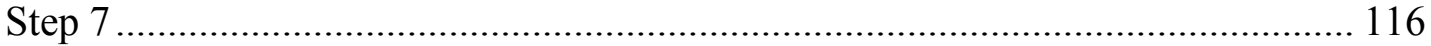

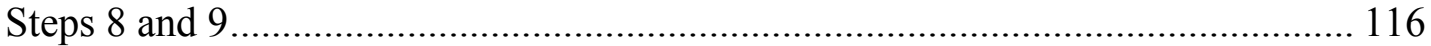

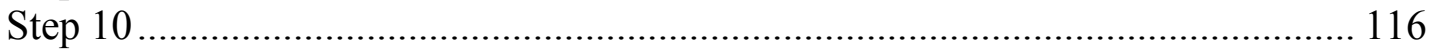

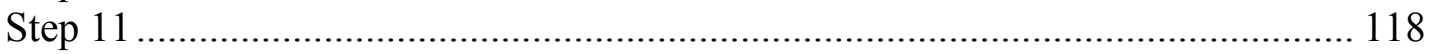

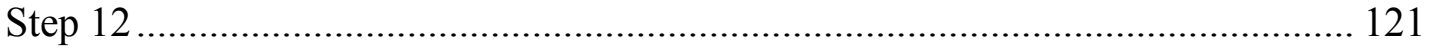

DATA PROCESSING (ROTATION) ............................................................... 121

APPENDIX F: VALIDATION OF DATA PROCESSING METHODOLOGY .... 123

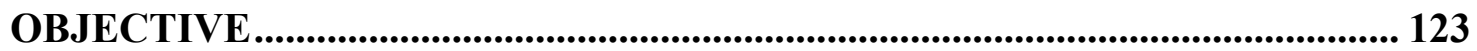

DESCRIPTION OF MODEL ........................................................................... 123

APPENDIX G: TABLES OF RESULTS ........................................................... 128

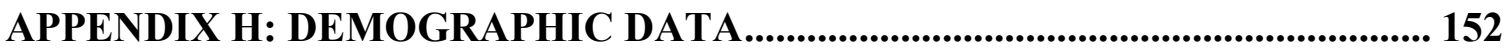

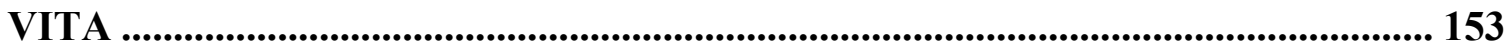




\section{LIST OF FIGURES}

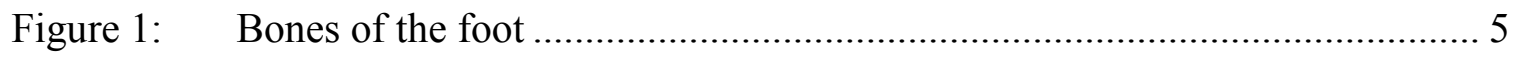

Figure 2: Medial and lateral views of talus of right foot ........................................... 6

Figure 3: Top view of talus of right foot ............................................................. 7

Figure 4: Talus, navicular and calcaneus bones ……………………………........... 9

Figure 5: Tibia and fibula of right leg (anterior view).............................................. 10

Figure 6: Ankle joint (anterior view) .................................................................... 12

Figure 7: Ankle joint (medial view) ………………......................................... 12

Figure 8: Subtalar joint (medial view) .................................................................... 14

Figure 9: Talonavicular joint (medial view) ……………………………………..... 15

Figure 10: Talonavicular joint (top view) ............................................................... 15

Figure 11: Calcaneocuboid joint (lateral view) ........................................................ 17

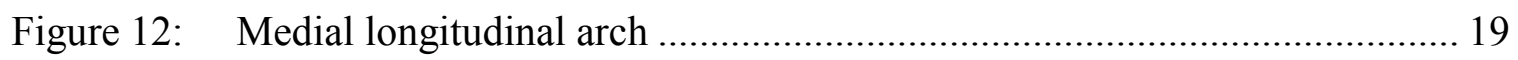

Figure 13: Lateral longitudinal arch .................................................................... 19

Figure 14: Transverse arch ............................................................................... 19

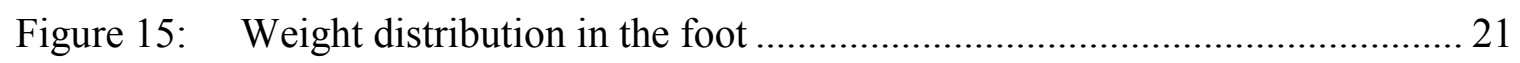

Figure 16: Orientation of the foot and plantar pressure pattern during parts of stance phase of gait cycle .............................................................................. 21

Figure 17: Three joints fused during triple fusion ................................................... 24

Figure 18: Test set-up (side view) ………………………………………….... 27

Figure 19: Test set-up (front view) ..................................................................... 28

Figure 20: Multiaxis programmable test frame - spine robot (left) four degrees of freedom (right) .................................................................................... 29

Figure 21: Motion tracking system: Dynasight ${ }^{\mathrm{TM}}$ camera (left) and three dimensional target mounted on a K-wire using clamps (right) .................... 31

Figure 22: Cable-puller enclosing the Achilles tendon ................................................ 33 
Figure 23: Target arrays mounted on K-wires inserted in bones.............................. 34

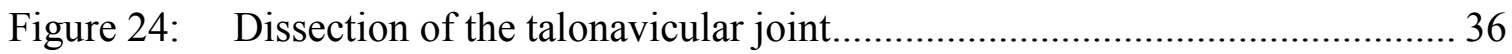

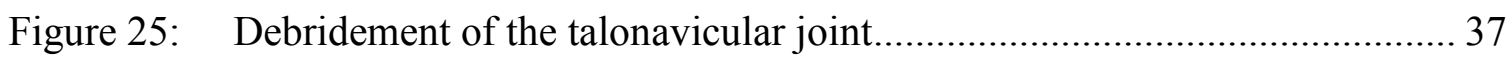

Figure 26: Point of insertion for medial screw .................................................... 38

Figure 27: Post-operative radiograph of two screws fixation technique (lateral

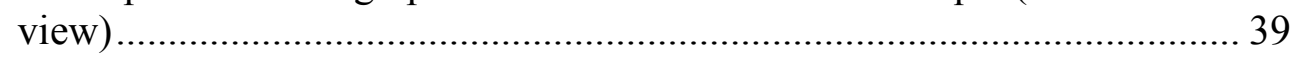

Figure 28: Post-operative photograph of specimen fixed using a locked compression plate plus one screw .................................................... 41

Figure 29: Post-operative radiograph of locked compression plate plus one screw (lateral view) ................................................................... 42

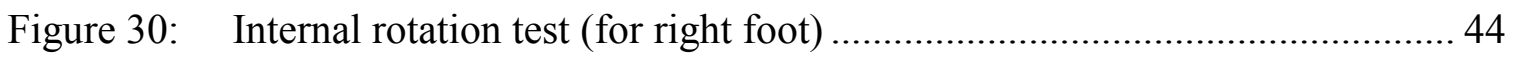

Figure 31: External rotation test (for right foot) …............................................ 45

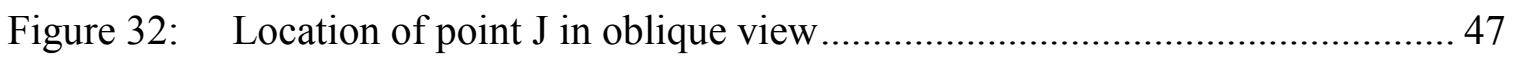

Figure 33: Location of point $\mathrm{J}$ in side view ....................................................... 48

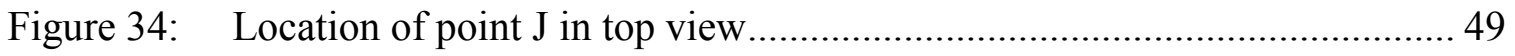

Figure 35: Reference frames (medial view) ......................................................... 51

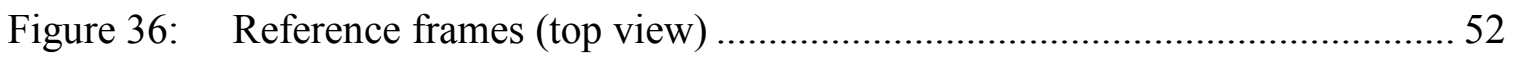

Figure 37: Relative translation along $\mathrm{X}$ axis (camera frame) .................................55

Figure 38: Relative translation along $\mathrm{Y}$ axis (camera frame) ..................................56

Figure 39: Relative translation along $\mathrm{Z}$ axis (camera frame) ….............................. 57

Figure 40: Relative rotation about $\mathrm{X}$ axis (camera frame) ….................................. 58

Figure 41: Relative rotation about $\mathrm{Y}$ axis (camera frame) …..................................59

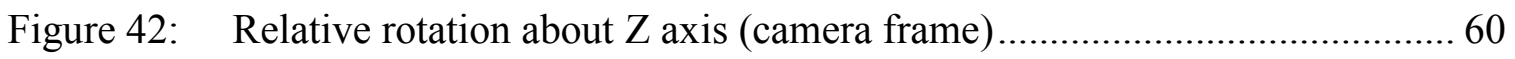

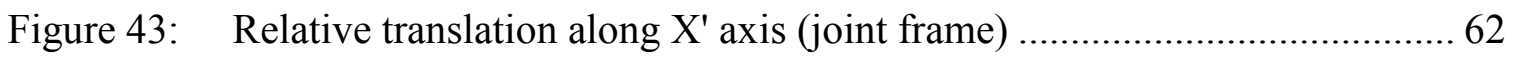

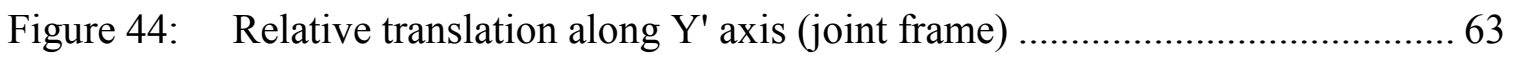

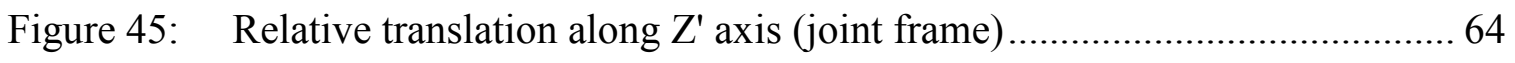




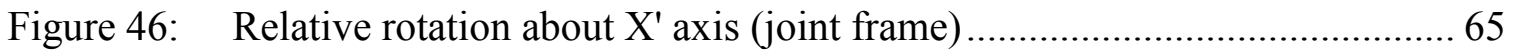

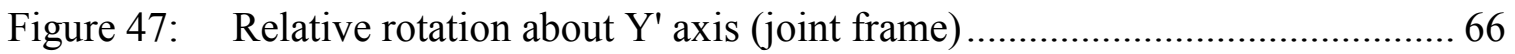

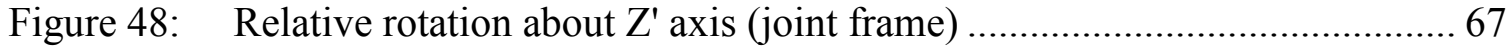

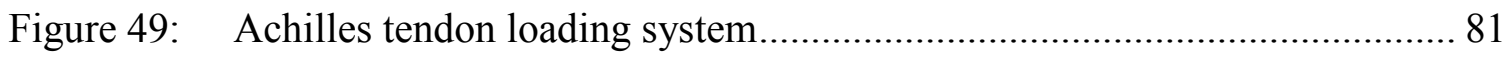

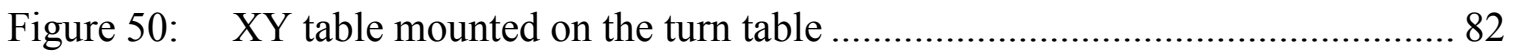

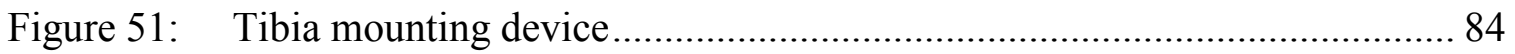

Figure 52: Tibia mounting device attached to load cell using mating plates ............... 85

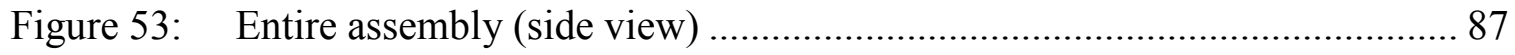

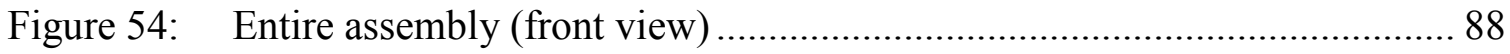

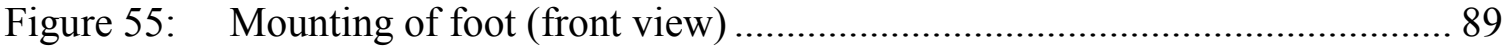

Figure 56: Mounting of foot (side view) ....................................................... 90

Figure 57: Lateral radiograph of foot showing XY location of point J ...................... 94

Figure 58: Top view of foot showing the depth of point $\mathrm{J}$ and distance from lower end of K-wire ........................................................................ 95

Figure 59: Schematic representation of pre-operative and post-operative location of point $\mathrm{J}$ (lateral view) ........................................................... 96

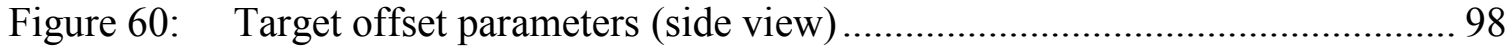

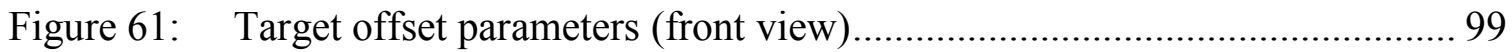

Figure 62: Diagrammatic representations of offset measurements (lateral view) ...... 100

Figure 63: Diagrammatic representations of radiographic offset measurements

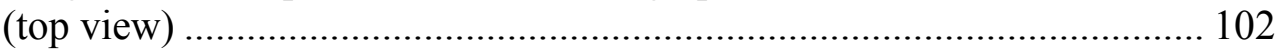

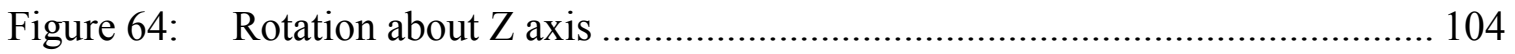

Figure 65: Point $\mathrm{K}$ is offset from point $\mathrm{T}$ by fixed distance .................................... 109

Figure 66: Coordinates of point $\mathrm{K}$ with respect to point $\mathrm{T}$ in camera frame .............. 110

Figure 67: Coordinates of J using coordinates of $\mathrm{K}$ and offset measurements........... 111

Figure 68: Global coordinates of $\mathrm{J}$ in the global camera system references from local target coordinates of talus target $\mathrm{T}$ 
Figure 69: Vectors from each target to point $J$..................................................... 114

Figure 70: Conversion of vectors into individual target reference frames .................. 115

Figure 71: Transformation of target coordinates into coordinates of J using

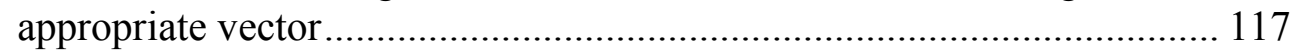

Figure 72: Joint frame orientation (medial view) ……………................................ 119

Figure 73: Joint frame orientation (top view) .......................................................... 120

Figure 74: Visual Nastran model showing two targets and point $\mathrm{J}$ at time zero ........ 124

Figure 75: Visual Nastran model showing the two targets and point $J$ at various

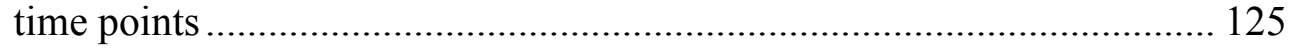

Figure 76: Visual Nastran model showing the validation of reference frame

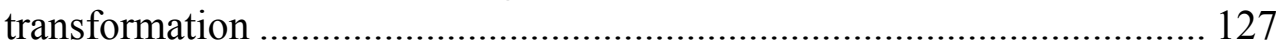




\section{CHAPTER 1: INTRODUCTION}

Human gait is a complex task involving interaction between the neural and musculoskeletal systems. The human foot is specially designed to carry out this task and withstand the enormous loads and moments occurring during gait. This is partly accomplished through the development of arches in the foot. The stability of the arches depends on the interaction between the bones, ligaments, muscles and tendons. The talus and navicular bones and the talonavicular joint play an important role in the stability of the medial longitudinal arch, in the weight distribution and in the motion of the foot.

The hind foot consists of three important joints namely the talonavicular joint, calcaneocuboid joint and subtalar joint. Of these, the talonavicular joint has the maximum mobility. The talonavicular joint is the main joint responsible for maintaining motion at other hind foot joints. Fusion of the talonavicular joint results in significant reduction of the other hind foot joint.[1]

The hind foot joints are affected by various diseases such as osteoarthritis, inflammatory arthritis, and post-traumatic arthritis. Failure of conservative modalities of treatment demands a more aggressive approach. Joint replacement techniques for hind foot joints are not popular since the subtalar, talonavicular and calcaneocuboid joints have small range of motion and are not suitable for standard joint replacement techniques. Thus arthrodesis is the mainstay of surgical treatment.[2] The fusion of talonavicular joint significantly reduces the motion of subtalar and calcaneocuboid joints.[1, 3] Hence there is a lot of interest in developing techniques for successful talonavicular fusion. The problem with isolated talonavicular fusion is the high rate of non union which has been reported variably from 3 to 37\%.[4-11] Consequently, a surgical technique of double or triple fusion i.e., fusion of more than one joint of the hind foot is used.

Ankle joint and midfoot joints may be secondarily affected after triple arthrodesis and show changes suggestive of arthritis apparent on radiograph.[12-15] Isolated joint arthrodesis may allow preservation of some hind foot motion thereby resulting in greater function. This may also help in better weight distribution and prevent ankle joint degeneration.[16] Hence, with the availability of modern internal fixation techniques, there is a renewed interest in techniques involving isolated talonavicular fusion. However there is a need to investigate the cause of the non-union, which is thought to be in part due to inadequate fixation. The exact biomechanics of hind-foot motion is not well understood, which is a problem in developing robust fixation techniques. Thus the goal of our study was to estimate the adequacy of talonavicular fixation and to elucidate biomechanics of the talonavicular joint.

There have been a few reported studies regarding the various fixation techniques used in clinical practice for treatment of talonavicular joint diseases. Jarrell et al[17] compared the effect of talonavicular fixation using a reconstruction plate, plate plus cancellous screw, and three screws. The specimens were loaded using axial compression. 
The pressure distribution in the foot was recorded. It was determined that all types of fixation resulted in lateralization of plantar pressure and plate/screw or screws would be similarly effective in producing talonavicular fusion. The positions of the individual bones were not recorded and thus the local biomechanics of the talonavicular joint were not studied directly. Instead the biomechanics of the joint were predicted based on global recording of plantar pressure patterns and only axial loads were applied to the foot. Thus the role of rotational movement coupled with the axial loading was not studied. Though their study established that fixation of the talonavicular joint leads to lateralization of plantar pressure, it did not investigate the stability achieved at the joint due to the fixation technique or instrumentation.

Rosenfeld et al[18] studied different fixation techniques for the talonavicular joint. They measured the talonavicular displacement for axial loads of $360 \mathrm{~N}$ and 12 cycles, with fixation of the joint using one or two talonavicular screws with or without subtalar fixation. Mean displacement of the navicular bone on the talus in the dorsal direction was reported to be less than $0.45 \mathrm{~mm}$. In this study, the joints were not debrided or destabilized in any way. Only displacement along one direction was reported. The study concluded that use of subtalar fixation screw increased the stability of talonavicular joint fixation. The study utilized the application of pure axial load. In reality, such isolated axial loading would rarely occur physiologically. Loading of the foot is accompanied by some degree of rotation such as adduction-abduction and inversion-eversion. The model developed in this study failed to directly assess the effect of such rotations. The model reported load versus displacement along a single axis and thus provided limited positional information. The study was also limited to various screw fixation techniques and did not investigate effect of any plate instrumentation.

Wulker, et.al have published papers regarding motion in the hind foot before and after arthrodesis as well as the peak pressures in adjacent joints after talonavicular fusion and triple fusion. $[1,2,19]$ They used ultrasonic trackers in a specimen suspended in space without weight bearing and inversion-eversion and other motion of foot was produced artificially. Results were rotations about global axes, out of which, the vertical axis was aligned with the long axis of tibia and one of the horizontal axes was aligned with the long axis of the foot and the third axis was perpendicular to the plane of these two axes. Mean rotations of about 15 degrees dorsiflexion-plantar flexion and 40 degrees of pronation-supination were reported. This finding was compared with other reports in literature which stated those values to be between 5 to 14 degrees for dorsiflexion-plantar flexion and 23 to 33 degrees for pronation-supination. A limitation of all these reported results was the use of a global reference frame which did not truly assess the actual rotation along the talonavicular joint plane. Also there was no weight bearing and thus any motion recorded can't be a perfect representation of the actual foot motion during gait. Lastly, these studies did not report any translational motion data.

To our knowledge there are no published studies describing rotational and translational motion in the talonavicular joint coordinate frame during weight bearing. Also, there are no published studies evaluating locked compression plates. Our study investigates the effect of fixation using a locked compression plate plus one lag screw 
versus with two lag screws on the translational and rotational motion at the talonavicular joint. Locked plates are relatively new technology for osteosynthesis. There are various advantages of locked compression plates including increased resistance to torsional forces, improved fixation in patients with poor bone quality, and the ability to provide two-column support. The screws in locked plates act in parallel so there is no toggle effect or loosening with repetitive loads. [20-23]

\section{OBJECTIVES OF THE STUDY}

1. To create a cadaveric foot model to be used in conjunction with a multi-axis programmable test frame.

2. To use the model to study the biomechanics of the talonavicular joint.

3. To compare the effect of two different fixation techniques on the joint biomechanics:

i. a locked compression plate plus one lag screw.

ii. two lag screws.

4. To investigate the relative translational and rotational motions between the talus and navicular bone in the talonavicular joint coordinate frame.

\section{NULL HYPOTHESIS}

There is no difference in the biomechanical stability of two different fixation techniques used for talonavicular fixation: a locked compression plate plus one screw versus two screws. 


\section{CHAPTER 2: BACKGROUND}

In order to design a foot model for biomechanical evaluation of the talonavicular joint, an understanding of the basic of the structure and functioning of the foot is essential. This chapter covers the basic anatomy and physiology of the foot.

\section{ANATOMY}

\section{Bones of the Foot}

There are 28 constant bones encountered in the foot (Figure 1). These include 7 tarsal bones, 5 metatarsals, and 14 phalanges. In addition, two sesamoid bones are often encountered in the foot. In addition to the above, tibia and fibula articulate with the talus to form the ankle joint and hence are described with the bones of the foot for the purpose of this study.

The seven tarsal bones occupy the proximal half of the foot and thus form what is often described as the hind foot and part of the mid foot. The main functions of the tarsals include transmission and distribution of weight in order to avoid direct transmission of weight from the tibia to the ground. For this purpose, the tarsals and the metatarsals are arranged in the form of longitudinal and transverse arches. The arches of the foot are described later in this document. The main bones of interest are described below.

\section{Talus}

The talus interfaces with the tibia and fibula to form the ankle joint and thus is the link between the shank and the foot. The talus, as seen in Figures 2 and 3, can be described as having a head, neck and body. The body is cuboidal, and the superior surface (trochlear surface) is shaped like a dome to articulate with the lower end of tibia. The medial and lateral surfaces articulate with the medial and lateral malleoli. The neck of the talus is narrow and has rough surfaces for ligaments. The head of the talus is directed distally and somewhat infero-medially. It has a distal convex surface which articulates with the proximal surface of the navicular to form the talonavicular joint. The talus articulates superiorly with the tibia to form the ankle joint, inferiorly with the calcaneus to form the subtalar joint and distally with the navicular and calcaneus to form the talocalcaneonavicular joint. There are no muscles attached to the talus but many ligaments are attached to it. 


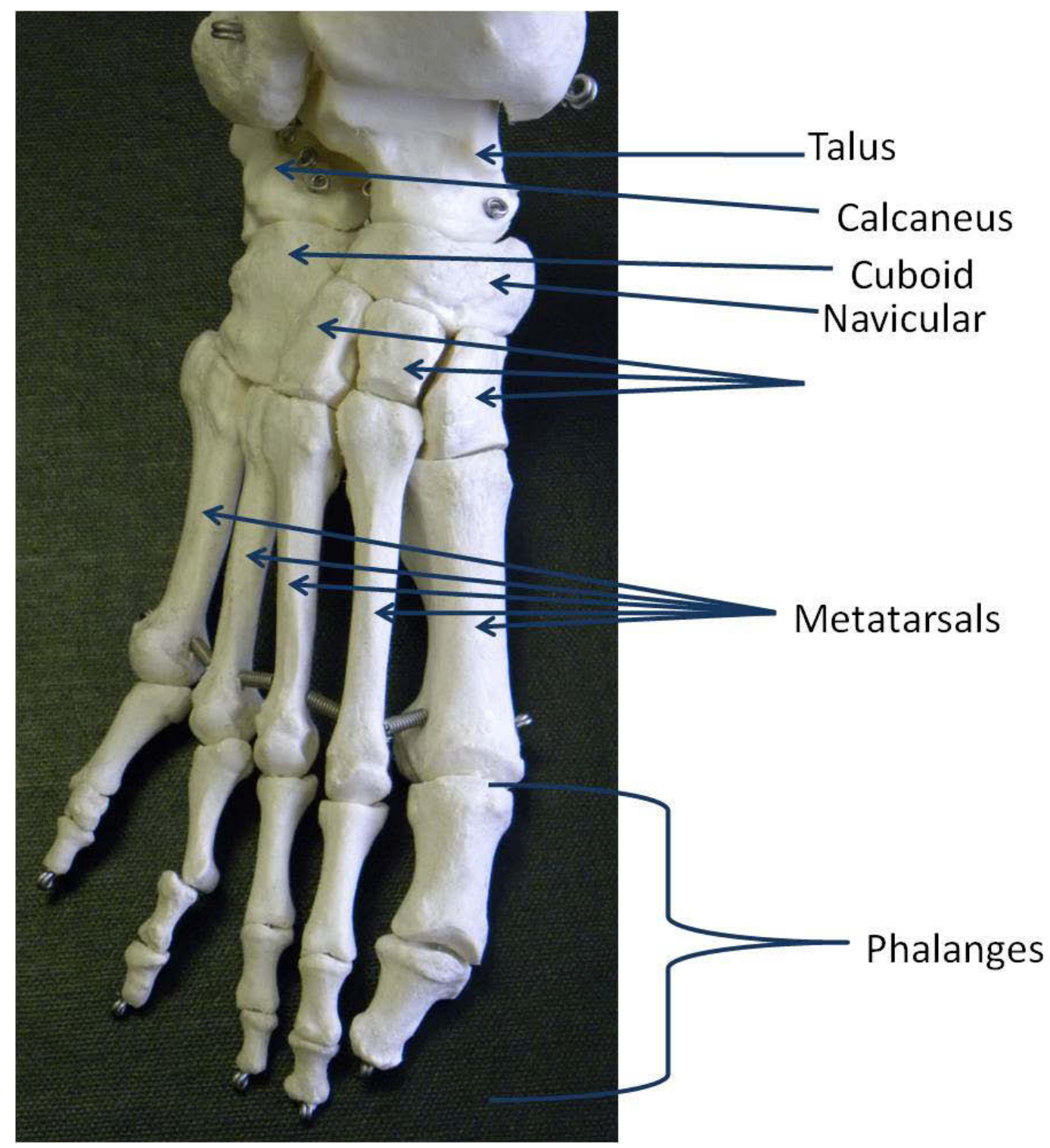

Figure 1: Bones of the foot 


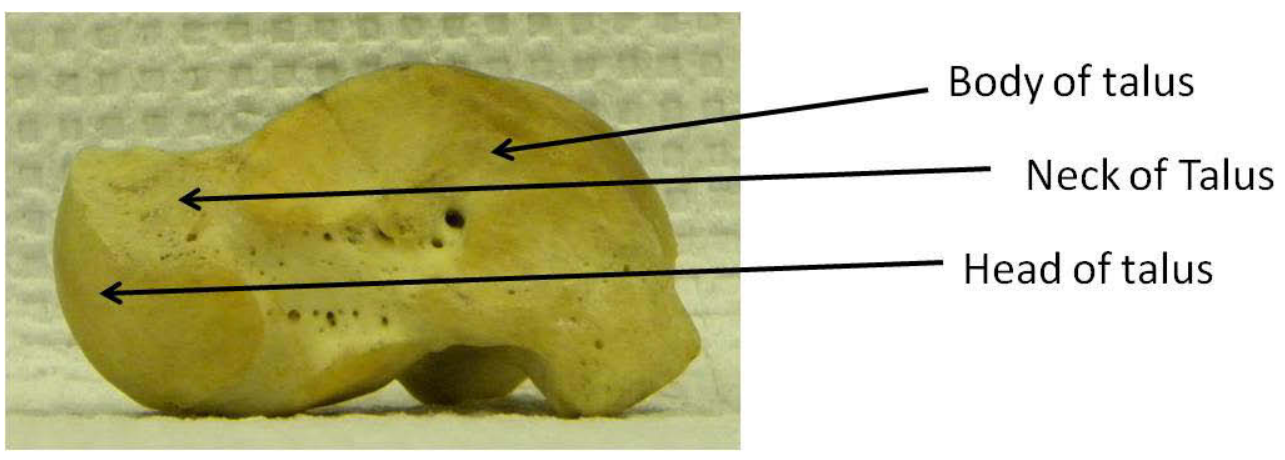

Medial View of Right Talus

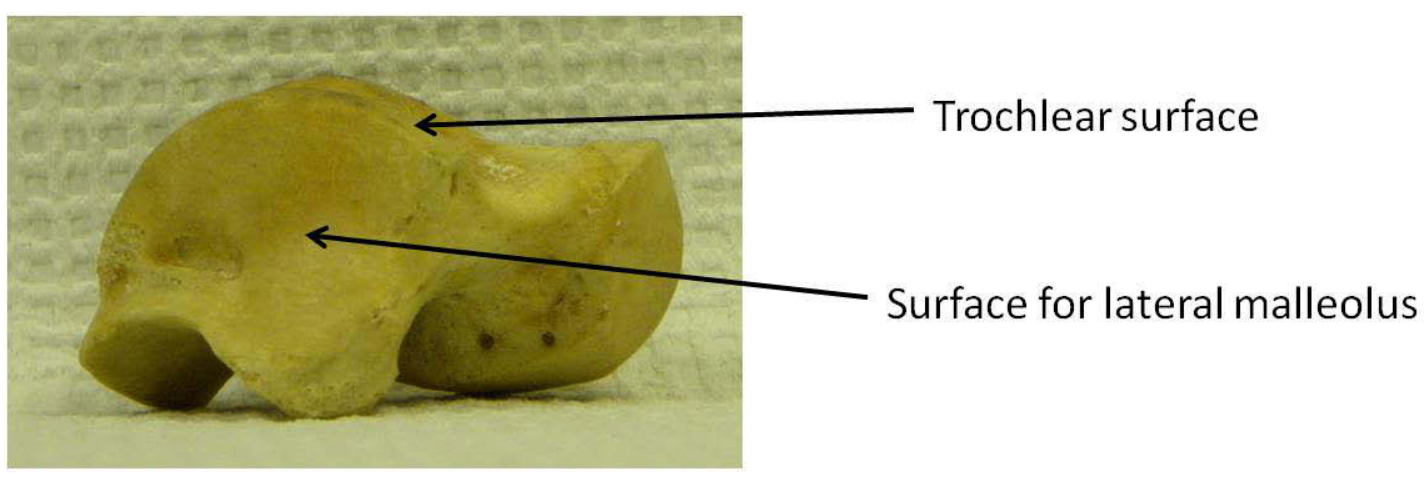

Lateral View of Right Talus

Figure 2: Medial and lateral views of talus of right foot 


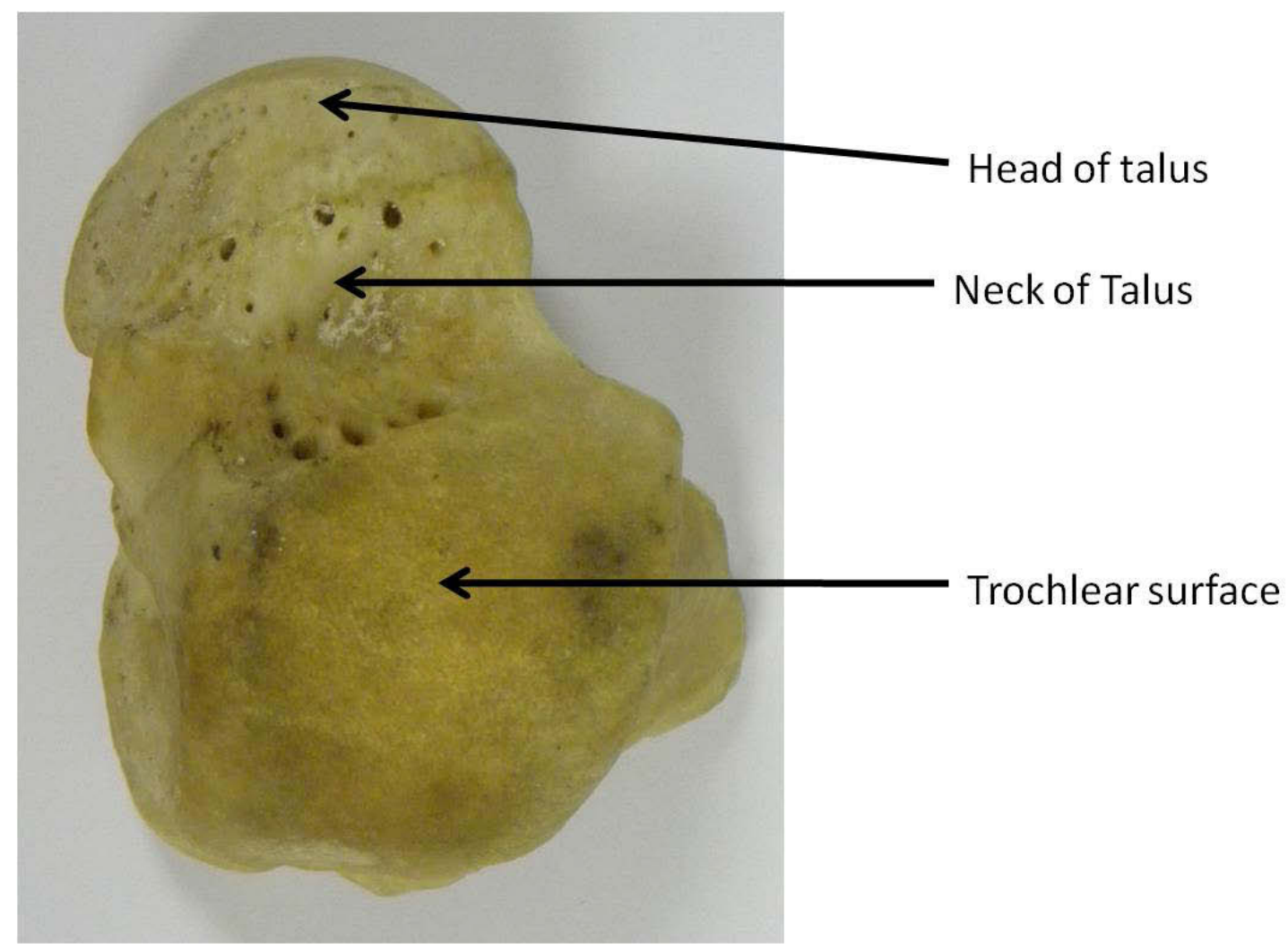

Figure 3: $\quad$ Top view of talus of right foot 


\section{Navicular}

The navicular bone (Figure 4) has a proximal concave surface for articulation with the talus, a distal convex surface which articulates with the medial cuneiform bones, and a lateral surface which often has a facet for articulation with the cuboid bone. The other surfaces are rough and are provide surface for attachment of various ligaments and tendons.

\section{Calcaneus}

The calcaneus (Figure 4) also known as the heel bone is the largest bone of the foot. It provides a posteroinferior projection to provide leverage for the muscles of the calf that are attached to it. The articulation of the calcaneus with talus and other tarsals varies. However for the purpose of the this study, it should suffice to say that, the calcaneus articulates with the talus superiorly to form the subtalar joint, distally with the talus and navicular to form the talocalcaneonavicular joint on the medial side, and with the cuboid bone to form the calcaneocuboid joint laterally. The calcaneus provides attachment for the Achilles tendon on the posterior surface and thus is involved in plantar flexion.

\section{Cuboid and cuneiform bones}

The cuboid and three cuneiform bones articulate with the calcaneus and the navicular bones proximally and the metatarsals distally to form the joints of the mid foot. They play important role in completing the arches of the foot which are vital for the stabilizing function of the foot.

\section{Metatarsals and phalanges}

The metatarsals are long bones located distal to the tarsals. Of particular note is the first or medial metatarsal which articulates with the proximal phalanx of the big toe. It is the shortest and thickest of the metatarsals and plays an important role in weight bearing. There are 14 phalanges in the five toes-two phalanges in the medial toe (big toe) and three phalanges in each of the four lateral toes.

\section{Tibia}

The tibia (Figure 5) is the medial bone of the leg. It is subcutaneous anteromedially. It is roughly triangular in cross-section and broader at the ends than the shaft. At the proximal end, it has two condyles and it articulates with the femur and patella to form the knee joint. At lower end it articulates with the fibula and the talus to form the ankle joint. The distal end of the tibia has a projection medially which can be 


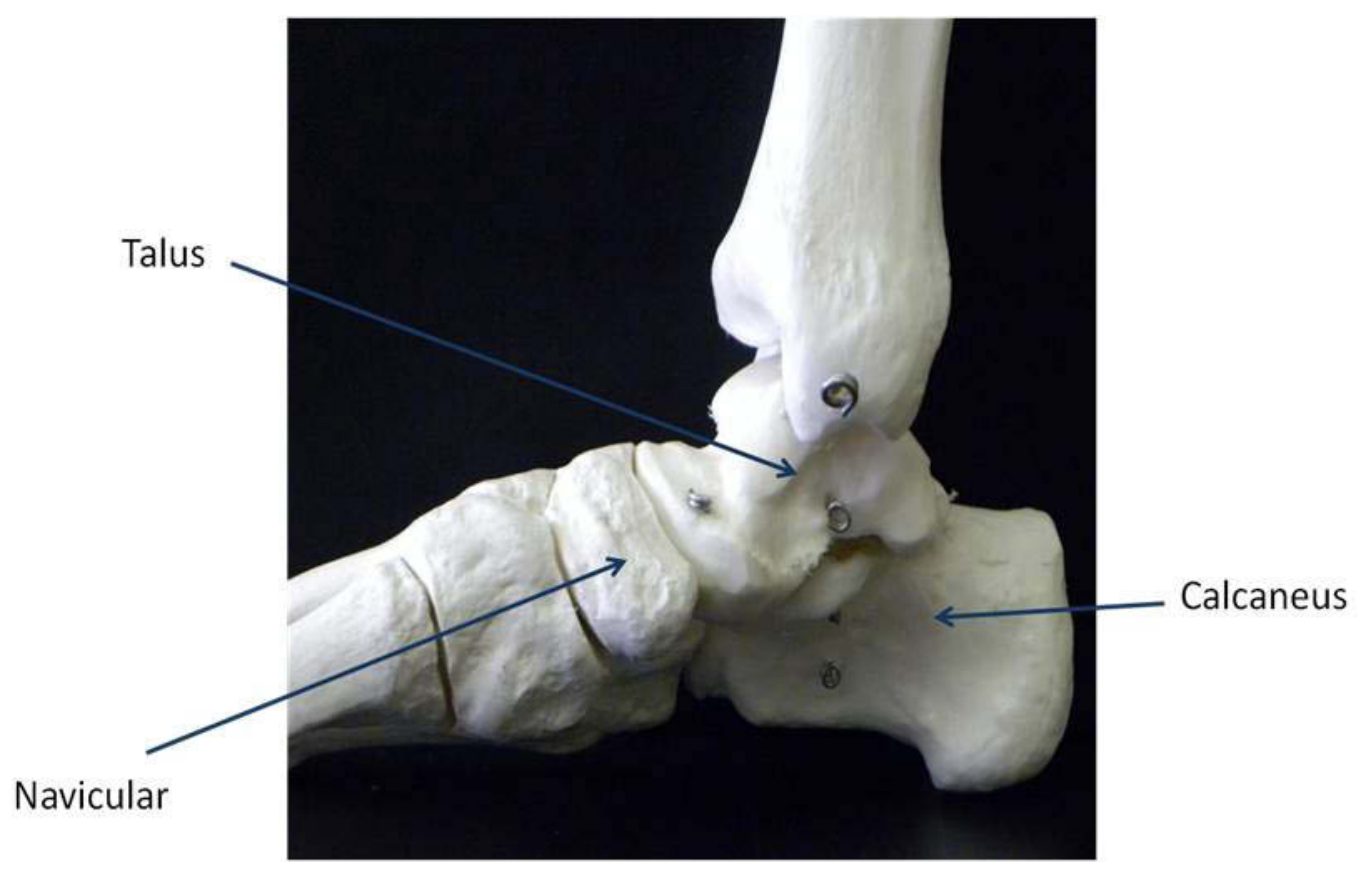

Figure 4: Talus, navicular and calcaneus bones 


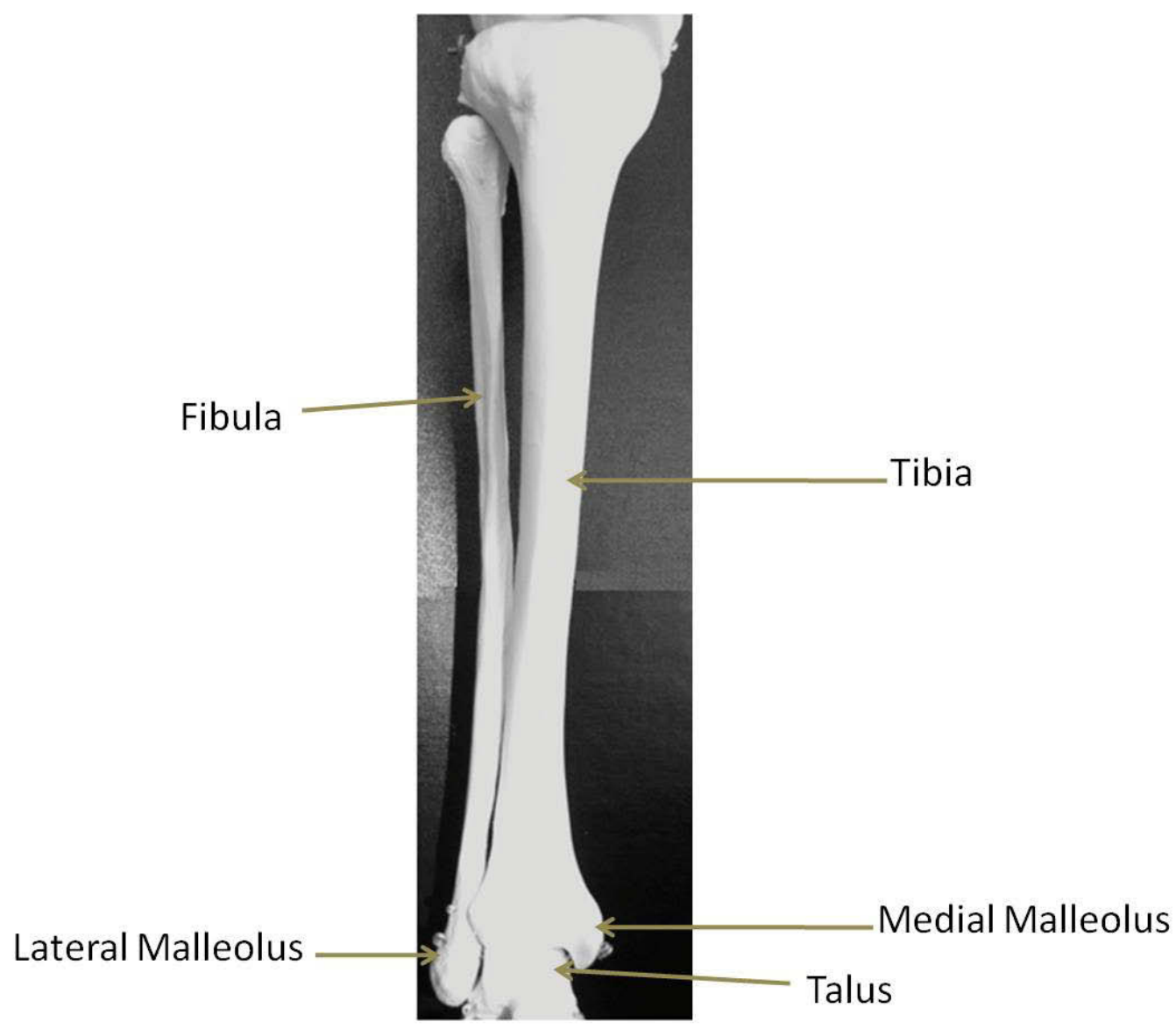

Figure 5: $\quad$ Tibia and fibula of right leg (anterior view) 
felt subcutaneously and called as medial malleolus. The distal end of tibia is slightly saddle shaped. It is wider in the front, concave sagittally and slightly convex transversely. It articulates with the dome of the talus bone.

The tibia provides surface for origin and attachment of multiple muscles. It is the weight-bearing bone and carries most of the body weight. This weight is transmitted to the talus and gets distributed to various areas of the foot.

\section{Fibula}

Fibula (Figure 5) is the lateral bone of the leg. It is slender. It has a head, neck and shaft and ends distally as the lateral malleolus. The head articulates with the lateral condyle of the tibia and the neck is closely related to the common peroneal nerve. The shaft provides surface for attachment of multiple muscles. The distal end projects as the lateral malleolus. It has a facet medially for articulation with the talus and provides attachment for multiple ligaments. The fibula is not directly involved in weight bearing and can be used for harvesting free vascularized diaphysis grafts.

\section{Joints of the Foot}

\section{Ankle (talocrural) joint}

The talocrural or the ankle joint (Figures 6 and 7) can be described as a hinge joint. The superior, medial and lateral surfaces of the body of the talus articulates with the inferior surface of the tibia, lateral surface of medial malleolus, medial surface of the lateral malleolus and inferior surface of the tibiofibular syndesmosis to form the ankle joint. The ankle joint is remarkable in its high degree of congruence and allows minimal mediolateral motion. The motion at the ankle joint is thus mainly dorsiflexion and plantar flexion. However there is gliding over the superior talar surface and hence the axis of dorsiflexion and plantarflexion doesn't coincide.

Passive stability is provided by the medial and lateral ligament complexes, the distal tibiofibular ligaments, the tendons, bony contours, and capsular attachments. Dynamic stability is provided by gravity, muscle action, and ground reaction forces.

Dorsiflexion is produced by tibialis anterior assisted by extensor digitorum longus, extensor hallucis longus and peroneus tertius. Plantar flexion is produced by gastrocnemius and soleus via the Achilles tendon and assisted by the plantaris, tibialis posterior, flexor hallucis longus and flexor digitorum longus. 


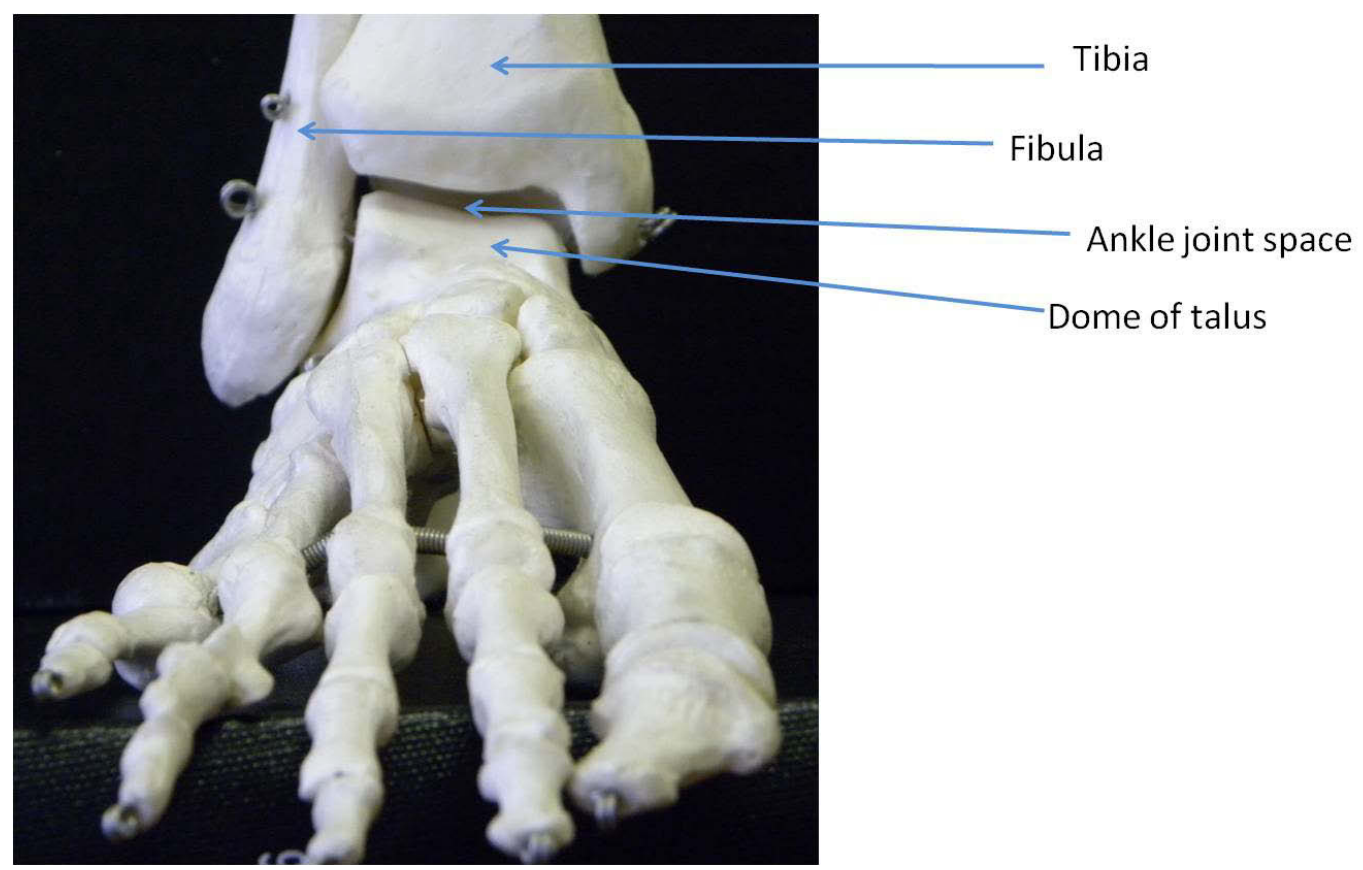

Figure 6: Ankle joint (anterior view)

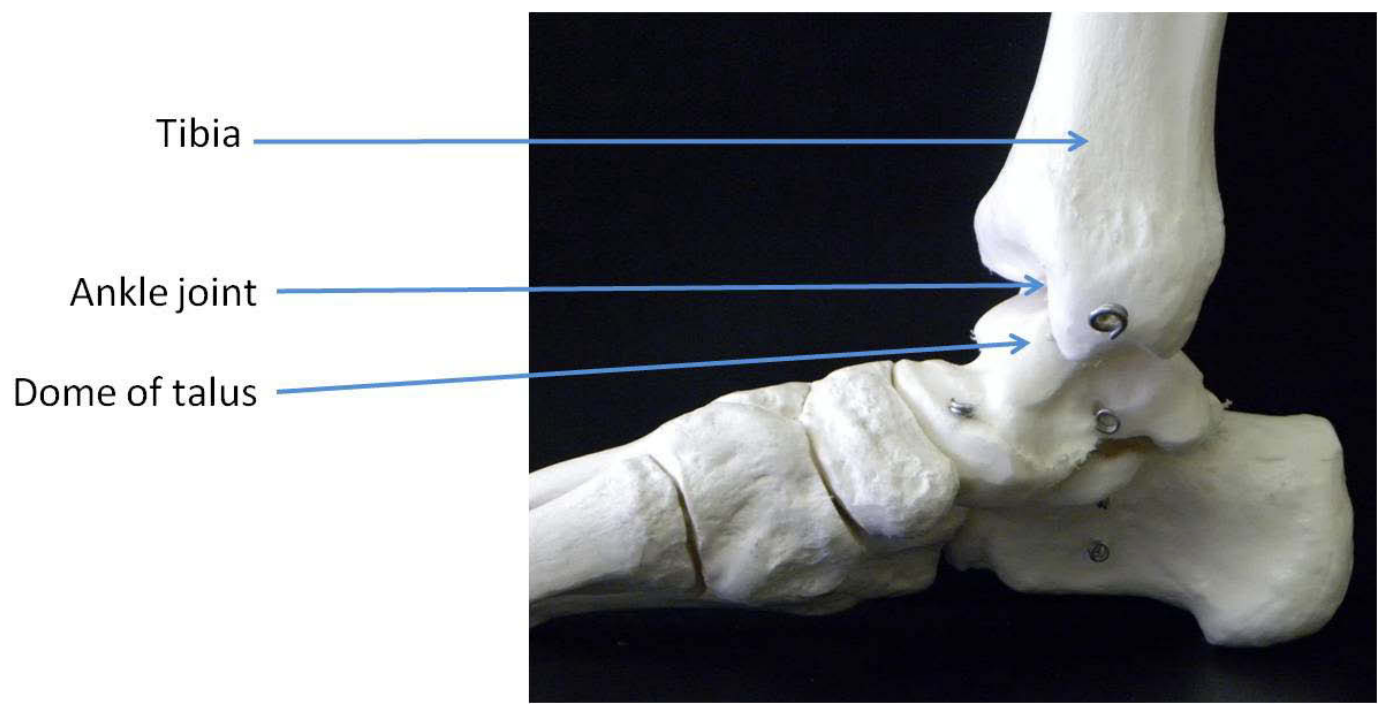

Figure 7: $\quad$ Ankle joint (medial view) 


\section{Subtalar joint}

The talus and calcaneus articulate anteriorly and posteriorly. The anterior joint is part of the talocalcaneonavicular joint and the posterior joint is called the talocalcaneal joint. However these two articulations form a single motion unit typically referred to as subtalar joint and is shown in Figure 8.

Stability for this joint depends on the ligaments including lateral and medial talocalcaneal ligament, interosseous talocalcaneal ligament, cervical ligament, and calcaneofibular component of the lateral ligament complex. However the relative contribution of each of these ligaments to the stability is not clearly known.

Heel inversion, which occurs mainly at this joint, is controlled by the tibialis anterior, posterior and gastrocnemius-soleus complex via the calcaneal tendon and flexors of the big toe. Heel eversion is produced by peroneus longus, brevis and tertius and extensors of the big toe.

\section{Talonavicular joint}

The talonavicular joint is at-times described as the talocalcaneonavicular joint, which includes the anterior part of the subtalar joint. The anterior part of the subtalar joint has already been described above and thus the following description relates to the talonavicular joint only. The medial view of the joint can be seen in Figure 9 and the top view in Figure 10.

The talonavicular joint is a synovial joint and is formed by the articulation between the distal convex surface of the head of talus and the proximal concave surface of the navicular bone. The articulating surfaces have a high degree of congruence and the bones are connected by the fibrous capsule, and three ligaments: talonavicular, plantar calcaneonavicular and calcaneonavicular part of the bifurgated ligaments.

The talonavicular ligament is a broad, thin band and connects the dorsal surfaces of the talar neck and navicular. It is covered by extensor tendons. The plantar calcaneonavicular ligament also known as spring ligament and the calcaneonavicular part of the bifurgated ligament are plantar and lateral ligaments of the joint respectively and play a role in maintaining the arches of the foot. The calcaneus doesn't directly articulate with the navicular but the calcaneonavicular ligaments act as the interface between these two bones. Thus the main bony interface is between the talus and navicular bones.

Factors responsible for stability are the bony contours and the ligaments. The fibrous capsule is poorly developed except posteriorly. All this indicates that the medial aspect of the joint is the least constrained.

Motion of the joint occurs in combination with the subtalar joint and is governed by the action of the same muscles as described above for the subtalar joint. The mean 


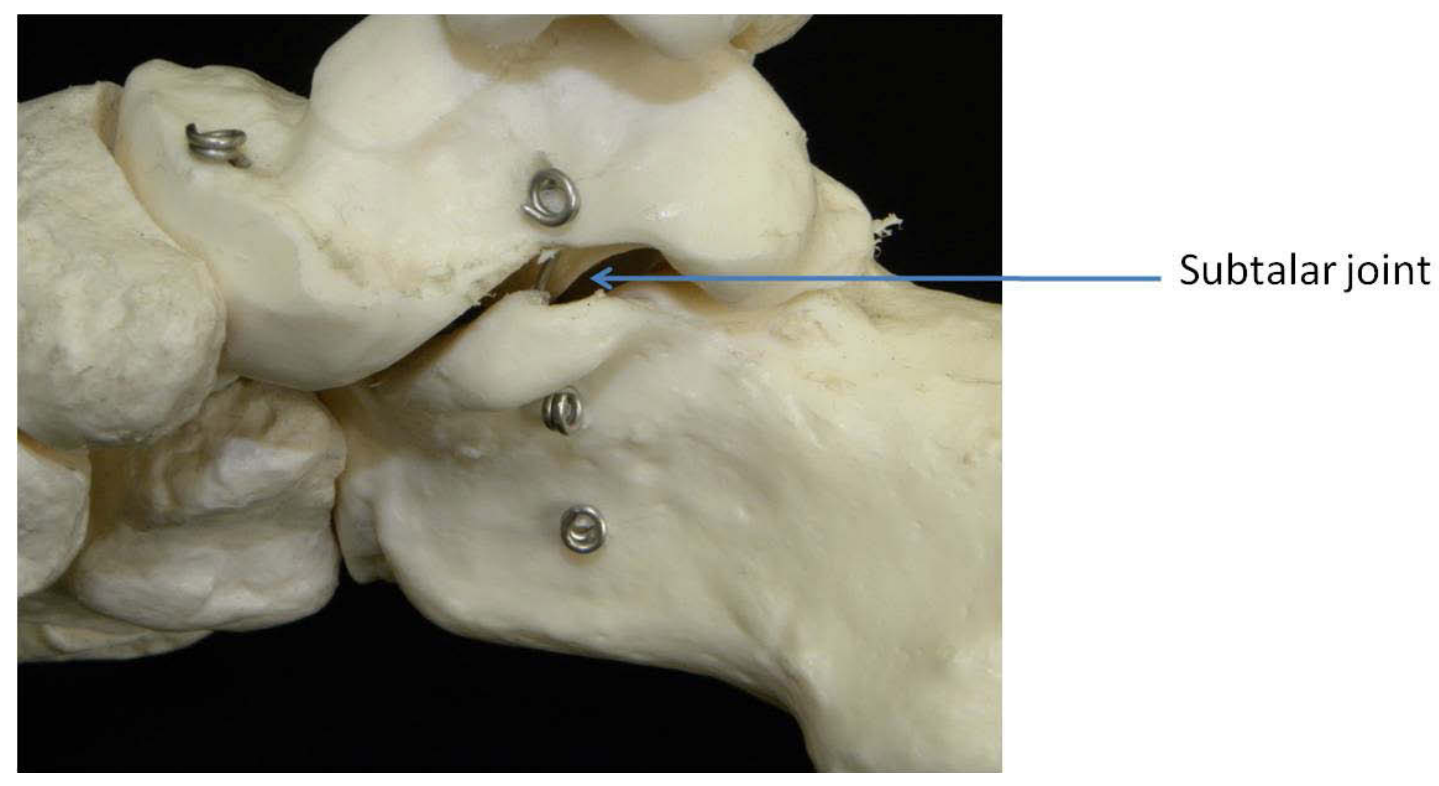

Figure 8: $\quad$ Subtalar joint (medial view) 


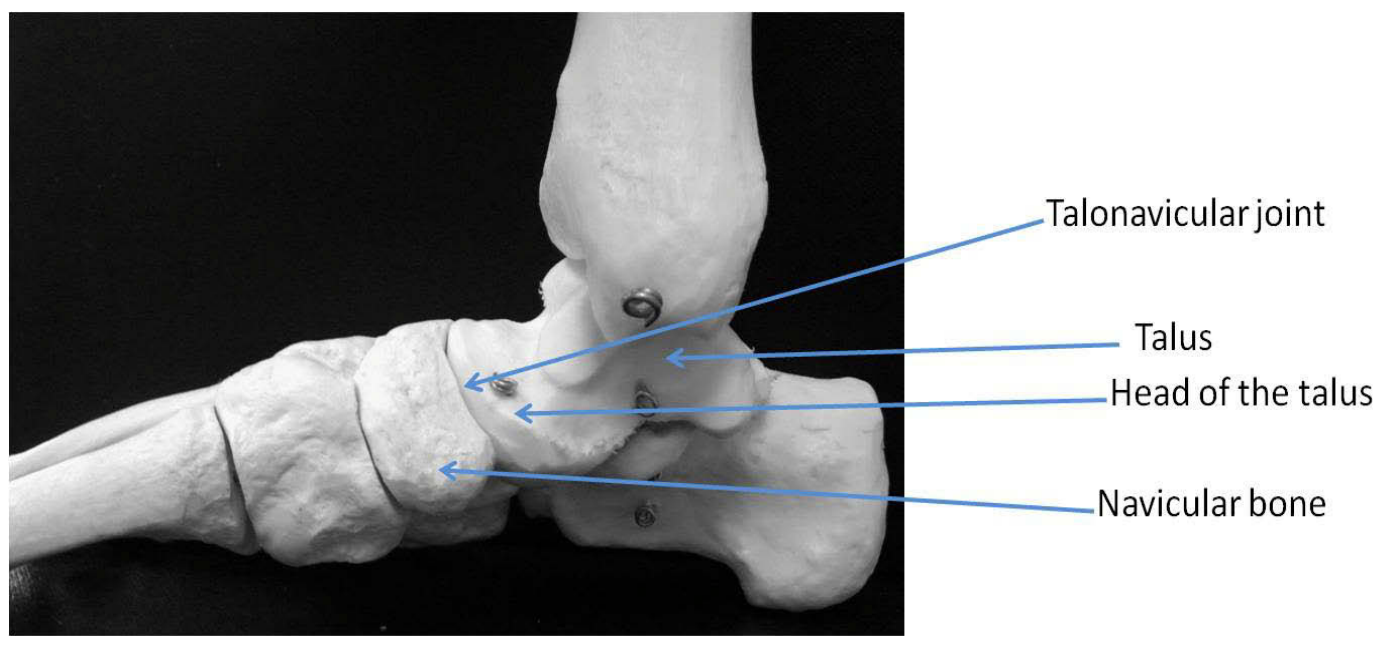

Figure 9: $\quad$ Talonavicular joint (medial view)

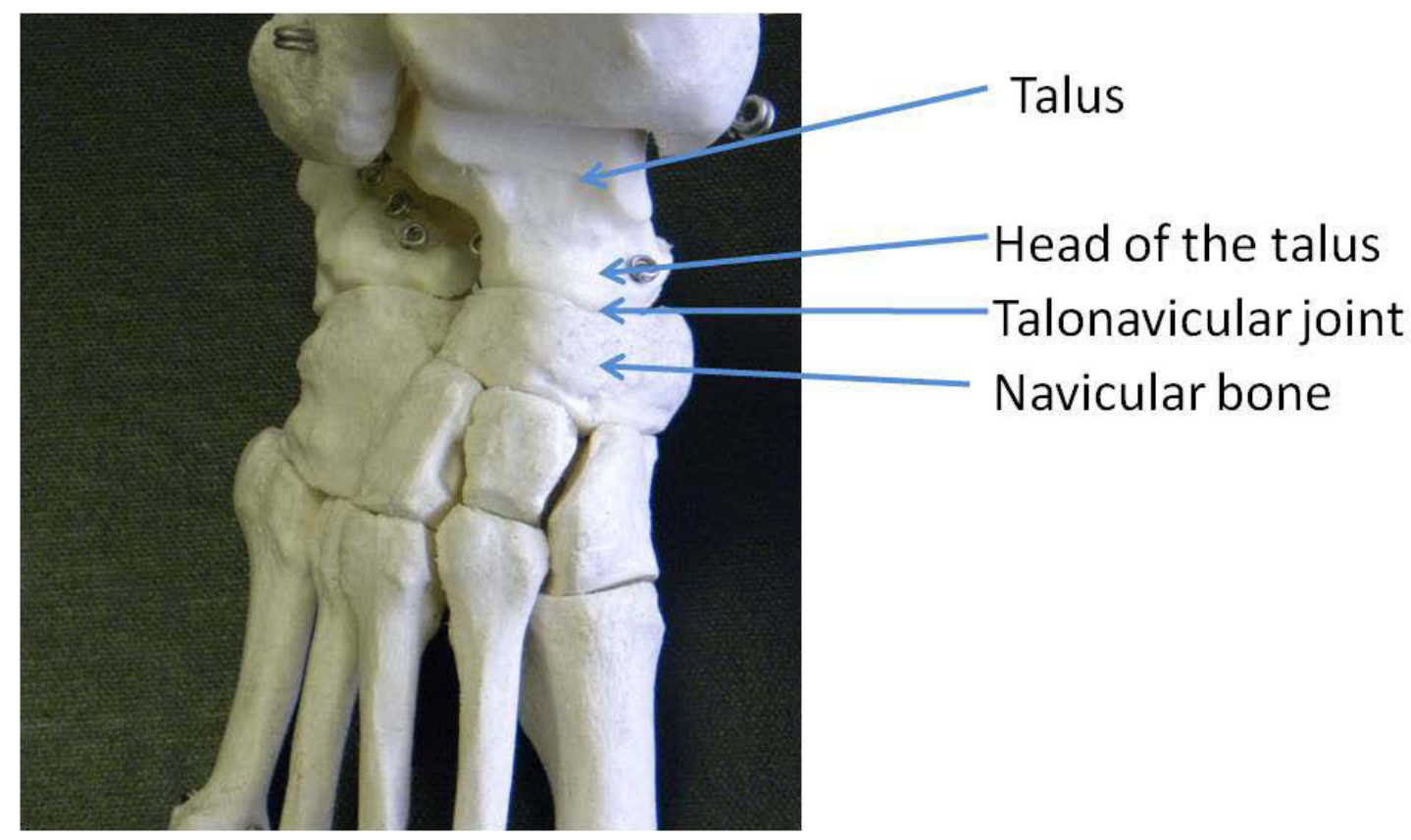

Figure 10: Talonavicular joint (top view) 
range of motion of the talonavicular joint has been variably reported to be between 5.4 to 18.6 degrees in plantarflexion-dorsiflexion and 23.8 to 40.7 degrees in pronationsupination.[1]

\section{Calcaneocuboid joint}

The calcaneocuboid joint (Figure 11), formed by articulation of anterior surface of the calcaneus and posterior surface of cuboid, is at the same level as the talonavicular joint and together these two are sometimes referred as the transverse tarsal joint. The calcaneocuboid joint allows small amounts of movements and is very stable due to the bony contours and ligaments including the bifurgated ligament, long plantar ligament, plantar calcaneocuboid ligament. The motion at this joint is of gliding type and related to inversion and eversion motion of the subtalar and talonavicular joints.

\section{Other joints of the foot}

Other joints of the foot include naviculocuneiform, intercuneiform, cuneocuboid, tarsometatarsal, intermetatarsal, metatarsophalangeal and interphalangeal joints. These joints play a role in the weight distribution and many of these are involved in forming the arches of the foot.

\section{Muscles, Tendons, Ligaments, Nerves, and Blood Vessels of the Foot}

Since this study was focused on the bones and joints in passive and in vitro state, the description of contribution of the muscles and ligaments does not fall in the purview of this study. Further, since it was an in vitro cadaveric study, the nerves and blood vessels were not significant factors in the study. The following is a brief description of the Achilles tendon which was the only soft tissue controlled during the study.

\section{Achilles Tendon}

The Achilles tendon, also known as tendo-achilles or calcaneal tendon, is formed from the triceps surae i.e., the two heads of the gastrocnemius and the soleus muscle. The plantaris also merges with the tendon.

The Achilles tendon is the thickest and strongest tendon in the human body and is about $15 \mathrm{~cm}$ long. It begins near the middle of the calf, though the initial section is covered by muscle fibers. In the lowermost $4 \mathrm{~cm}$, it is rounded and then expands to attach to the posterior surface of the calcaneus. The fibers of the tendon are not vertical, but they rotate spirally to a variable degree. 


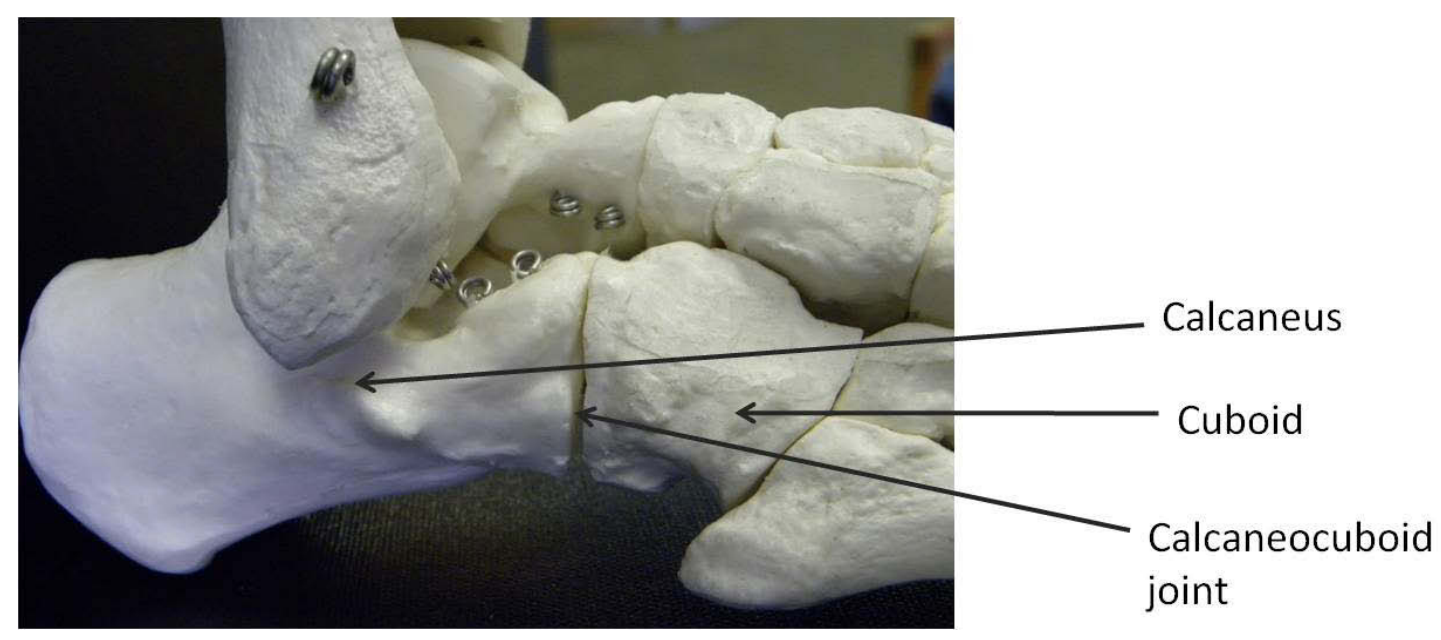

Figure 11: Calcaneocuboid joint (lateral view) 
The main action of the Achilles tendon is plantar flexion of the ankle joint. Due to spiral arrangement of the fibers, the fibers from gastrocnemius are attached more laterally than the soleus. This provides a moment at the point of insertion causing plantar flexion to be coupled with internal rotation/inversion of the foot on the tibia. The Achilles tendon can carry immense amount of load. It has been estimated that the load in the Achilles tendon may be 2.1 times the body weight.[24]

\section{ARCHES OF THE FOOT AND WEIGHT DISTRIBUTION}

The foot has been described to contain three arches, namely the medial longitudinal arch, lateral longitudinal arch, and the transverse arch.

\section{Medial Longitudinal Arch}

The medial longitudinal arch (Figure 12) is made up of calcaneus, talus, navicular, three cuneiforms and their three metatarsals. The pillars are posterior aspect of the calcaneus and the three metatarsal heads. The talus bone appears to be the key stone of this arch, though the contribution of the bones to the stability of the arch is disputed. The stability of the arch is explained using the theory of windlass effect[25] and the ligaments and muscles are postulated to be the major contributors to the stability of the arch.

\section{Lateral Longitudinal Arch}

The calcaneus, cuboid and the fourth and fifth metatarsal make up this arch which again depends on the ligaments for the stability. The lateral longitudinal arch is shown in Figure 13.

\section{Transverse Arch}

The bases of the five metatarsals, the cuboid and cuneiform bones make up this arch. The intermediate and lateral cuneiform are wedge shaped and thus help to maintain this arch. The ligaments and muscle tendons are also major contributors to the stability for this arch. The transverse arch is shown in Figure 14.

\section{Importance of Arches of the Foot}

The foot may experience forces much higher than the body weight. For example, during running, the peak vertical forces approach $275 \%$ body weight.[25] In the absence of the arches this force would compress and compromise the blood supply and nerves. The arches distribute the body weight and prevent compression of neurovascular bundle. 


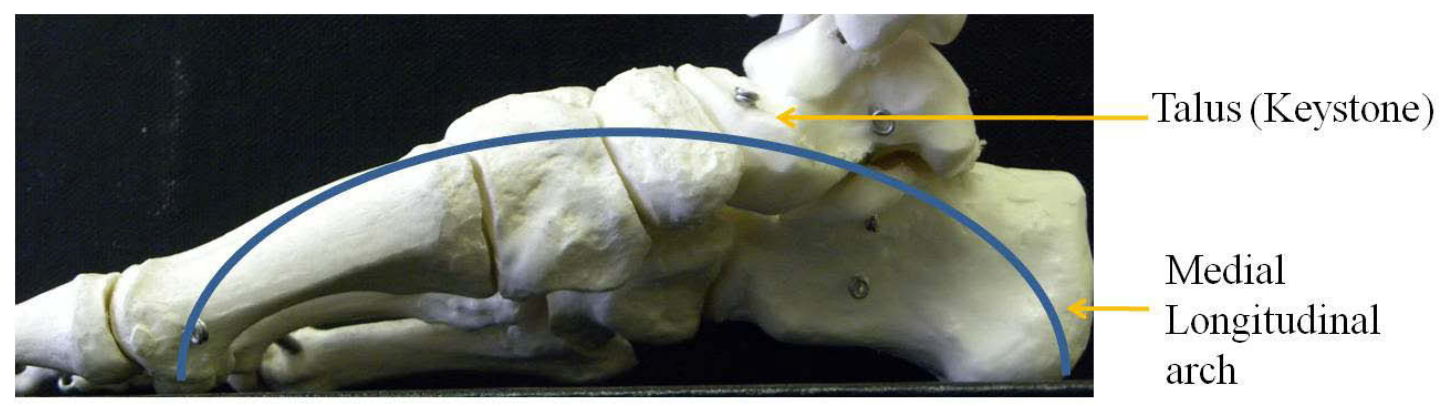

Figure 12: Medial longitudinal arch

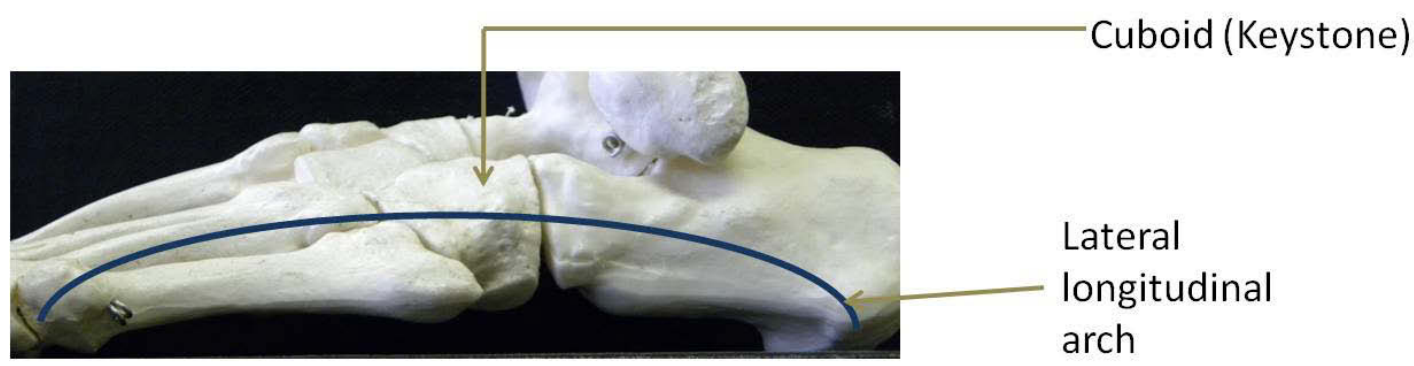

Figure 13: Lateral longitudinal arch

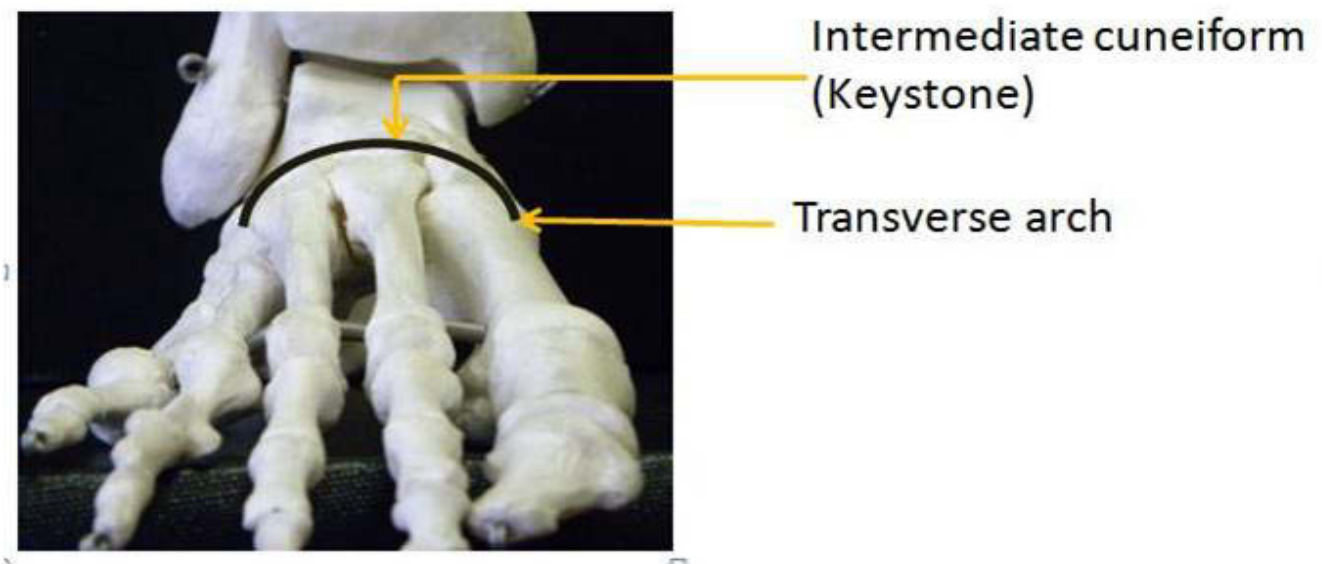

Figure 14: Transverse arch 
Arches allow shock absorption and proper weight distribution. The body-weight borne by the foot is not distributed evenly across the entire sole (Figure 15). Instead, weight is mainly borne by the heel bone and the head of the $1^{\text {st }}$ metatarsal bone along with some diffuse weight bearing by the lateral edge of the foot. In standing position approximately $60 \%$ of the body weight is borne by the heel while about $28 \%$ is borne by the forefoot. The rest is borne by the lateral edge of the foot and lateral metatarsals.[25]

\section{GAIT}

Human gait has been the subject of various studies. Gait is the result of complex interaction between bones, muscles and ligaments which allows a wide range of movement patterns. Though a detailed description of human gait is beyond the scope of this document, the main aspects which may have a bearing on this study are described below. Unless stated otherwise, the events described are those occurring during normal walking gait.

Gait of each foot can be described to consist of two phases which repeat cyclically and hence the term gait cycle. The two phases are stance phase during which, at least some part of the foot is in contact with the ground and swing phase during which, no part of that foot is in contact with the ground. In normal walking, at any given instant, at least some part of at least one foot is in contact with the ground.

For the purpose of this study, the stance phase of gait was of interest. The stance phase can be subdivided into heel strike, foot flat, heel rise, push-off, and toe-off, which describe the sequence of events from the point of initial contact of the foot with the ground at the end of previous swing phase till the beginning of the next swing phase. The parts of stance phase of gait and plantar pressure patterns are depicted in Figure 16.

During heel strike, the contact point of the foot is at the heel and the body weight starts getting transferred from the other foot to the foot under observation. The point of contact rapidly moves forward as the anterior part of the sole comes in contact with the ground and the body weight is shared between the heel and the forefoot. This is called foot flat phase. During the next phase, the body weight is transferred to the anterior part of the sole thus preparing the foot for heel rise phase during which the heel is not in contact with the ground and the entire body weight is on the forefoot and lateral surface of the sole. This is followed by push-off, during which the body is propelled forward by pushing on the ground with the contact area reduced to the forefoot. The last part of the stance phase is toe-off, during which the major part of the body weight is borne by the contra-lateral foot while a small portion of the body weight is entirely borne by the head of the first metatarsal and the ball of the big toe. The foot then continues into swing phase during which no part of the foot is in contact with the ground and this is followed by heel strike and the entire cycle repeats.

During the initial $15 \%$ of stance phase the entire lower extremity including the pelvis, femur and tibia rotate internally with respect to the foot. From heel strike to foot 


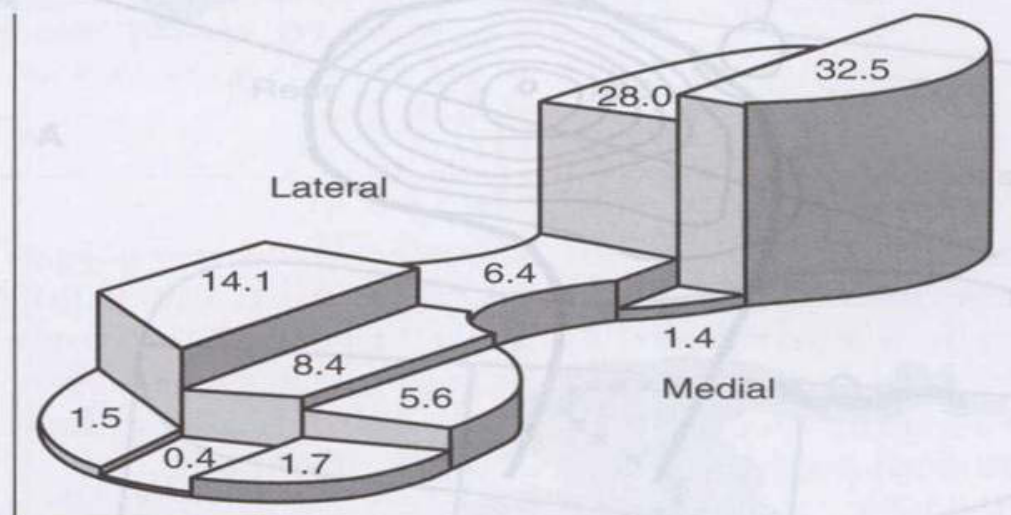

\section{FIG. 9-38}

Mean regional weight distribution expressed as a percentage of total load carried by the foot in barefoot standing. Over $60 \%$ of the weight is distributed in the rearfoot, $8 \%$ in the midfoot, and $28 \%$ in the forefoot. The toes have little involvement in the weight-bearing process.

\section{Figure 15: Weight distribution in the foot}

Note: Reprinted with permission. Nordin M, Frankel VH. Basic biomechanics of the musculoskeletal system. Philadelphia: Lippincott Williams \& Wilkins; 2001, page 239.[25]

Heel Strike

Figure 16: Orientation of the foot and plantar pressure pattern during parts of stance phase of gait cycle 
flat phase, the subtalar joint everts, the foot pronates and becomes more flexible to absorb shock and adapt to irregularities of the ground. During the mid-stance phase to push-off phase the entire situation reverses. The entire lower extremity rotates externally with respect to the foot while the subtalar joint inverts transforming the foot into a rigid structure capable of propulsion. 


\section{CHAPTER 3: PATHOLOGY AND TREATMENT}

\section{HIND FOOT PATHOLOGY}

The hind foot pathology as related to this project is described in this section. The talonavicular, subtalar and calcaneocuboid joints are considered to be the hind foot joints. Degenerative changes in these joint may be primary or secondary. Primary degeneration due to osteoarthritis is rare in the subtalar joint but may occur in the transverse tarsal joints, i.e., the talonavicular and the calcaneocuboid joints. The talonavicular joint is the most common site of idiopathic hind foot arthrosis, inflammatory and rheumatoid arthritis.[2, 7] All three joints may also develop secondary arthritis following trauma or may be involved in rheumatoid or other generalized arthritic conditions.

The patient usually complains of pain, tenderness or soreness in the ankle joint during walking. However on physical examination, the ankle joint is often found to be normal and painless. There may be limitation of inversion-eversion and/or tenderness over the talonavicular joint. There may be peroneal spasm on inversion.[7] In advanced arthritis, significant deformity of the talonavicular joint may be observed. In this the head of the talus tends to drop in a plantar and medial direction. The calcaneus develops a valgus deformity secondary to this and the forefoot is abducted together constituting an acquired flatfoot deformity. Radiographic examination may reveal a normal ankle joint in addition to irregularity and narrowing of the talonavicular joint space.[7] The symptoms and findings in cases of osteoarthritis of the talonavicular joint are similar to rheumatoid arthritis. However there is frequently a history of injury since osteoarthritis is usually secondary to trauma. There may be tenderness on the talonavicular joint and radiographs may show loss of joint space, presence of subchondral sclerosis, hypertrophic bone formations and bone cysts. Since osteoarthritis is mostly asymptomatic, diagnosis must be based on correlation between findings of physical examination and radiographs. Isolated radiographic evidence of osteoarthritis must not be considered as the primary pathology. Differential diagnoses include sprains, enthesitis or tendonitis of the tibialis posterior, fractures, gout, and referred pain.[26]

\section{TREATMENT}

Treatment includes conservative and surgical treatment. Conservative treatment includes pain medications, physiotherapy, shoe modifications and heat therapy to control the symptoms. A polypropylene ankle foot orthosis with the trim line of the brace modified to permit $50 \%$ ankle motion may also be used.[27]

Failure of conservative treatment warrants the use of surgical treatment. Surgical treatment includes fusion of the talonavicular, subtalar and calcaneocuboid joints, often referred to as triple fusion. The three joints fused during this procedure are seen in Figure 17. The procedure is technically demanding. During triple fusion the subtalar joint is fused in 5 degrees valgus and the talonavicular and calcaneocuboid joints are 


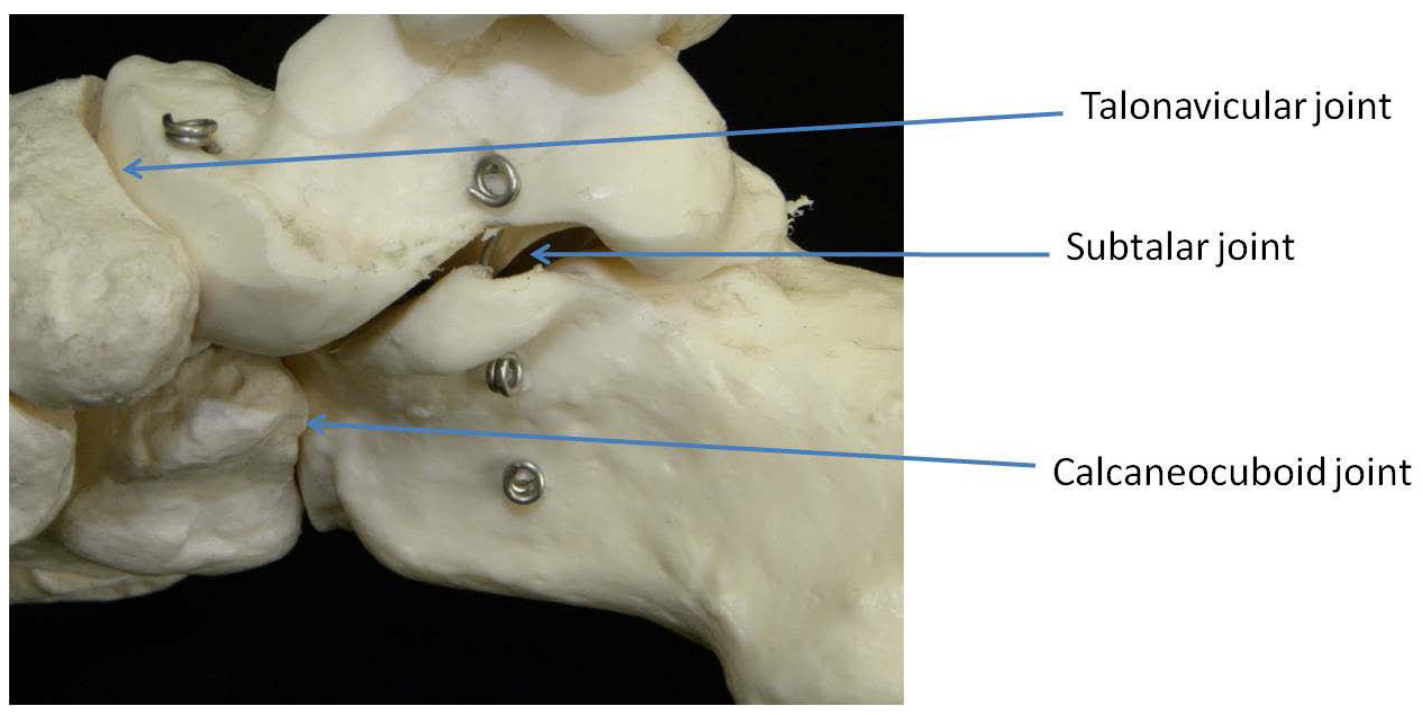

Figure 17: Three joints fused during triple fusion 
fused in neutral orientation avoiding excessive pronation or supination. Optimal positioning of the subtalar and transverse tarsal joints is necessary to create plantigrade foot to avoid abnormal weight bearing. After fusion the patient can carry on daily activities. However the extra stress on the ankle joint may create a problem. The pronation-supination (inversion-eversion) motion is lost and only ankle joint motion in the form of flexion-extension remains after successful triple fusion.[28]

In order to avoid the various problems associated with triple fusion such as arthritis of the adjacent joints (such as ankle and mid-foot joints), osteoporosis, limitation of ankle dorsiflexion, $[12-15,28]$ double fusion (fusion of two out of three joints) or isolated talonavicular fusion is used. The union rates of isolated talonavicular joint fusion procedures have been traditionally described to be lower than triple fusion and ranging from 63 to $97 \%$.[4-11] Talonavicular joint fusion is simpler since the subtalar joint need not be fused.[28]

Indications of talonavicular joint fusion include osteoarthritis, inflammatory arthritis, post-traumatic arthritis, posterior tibial tendon insufficiency (PTTI), acquired flatfoot deformity, rheumatoid arthritis, collapse secondary to rheumatoid involvement of spring ligament and neuromuscular instability.[28, 29]

The standard method of fixation used clinically involves insertion of two screws in a retrograde manner.[18,28] A newer approach is to use a locked compression plate in conjunction with a single lag screw. The locked plate creates a rigid fixed angle construct that does not require compression of the plate to bone for stability, which is imperative for traditional non-locking plate and screw constructs. Locked plates are also referred to as internal external fixators because they are more biomechanically similar to external fixation devices. Advantages of fixed angle constructs include increased resistance to torsional forces, improved fixation in patients with poor bone quality, and the ability to provide two-column support. The screws in locked plates act in parallel so there is no toggle effect or loosening with repetitive loads.[20-23] The details of the surgical procedure used in this study have been described later in this thesis. 


\section{CHAPTER 4: MATERIALS AND METHODS}

This study was done by adapting the multi-axis programmable test frame to test biomechanics of the foot. A turn table, XY table, a device for loading the Achilles tendon and various other fixtures were used. The entire assembly is seen in Figures 18 and 19.

\section{INSTRUMENTS AND FIXTURES}

\section{Multiaxis Programmable Test Frame (Spine Robot)}

The multi-axis programmable testing apparatus popularly known as spine robot, (Figure 20) was developed by Brian Kelly, Ph.D. and Denis DiAngelo, Ph.D. at the University of Tennessee Health Science Center, Memphis, TN. The robot provides multi-axis displacement control, force control, or hybrid control. It has four degrees of freedom ( 2 translational axes and 2 rotational axes). It has been successfully used in multiple protocols to test various spine instrumentations used for spinal fusion and spinal disc replacement. As a part of this study, the robot was adapted by creating and using various fixtures and specific program to test foot biomechanics.

A feature of this robot is the ability to program tool-tip transformations about a user defined virtual point in space resulting in multi-axial movement around this point. This allowed precise control of motion and loading while monitoring the loads at a user defined point near the ankle joint.

\section{XY Table and Turn Table}

An XY table was designed and created using two low friction slides with appropriate mating plates. This table allowed motion of the foot specimen in the XY plane thus reducing the constraints on the model. On application of any load to the foot, the foot was free to move in the XY plane and assume any orientation. This allowed the rotation of the foot to occur about its natural center of rotation instead of a user defined point. The XY table was mounted on a turn table which was capable of providing rotation with a torque of up to $20 \mathrm{Nm}$. The design of the $\mathrm{XY}$ table is explained in Appendix A.

\section{Achilles Tendon Loading System}

The Achilles tendon was loaded during this study using a predefined dead weight. A device was designed and created which allowed loading of the Achilles tendon in near physiological orientation. The design of this device is explained in Appendix A. 


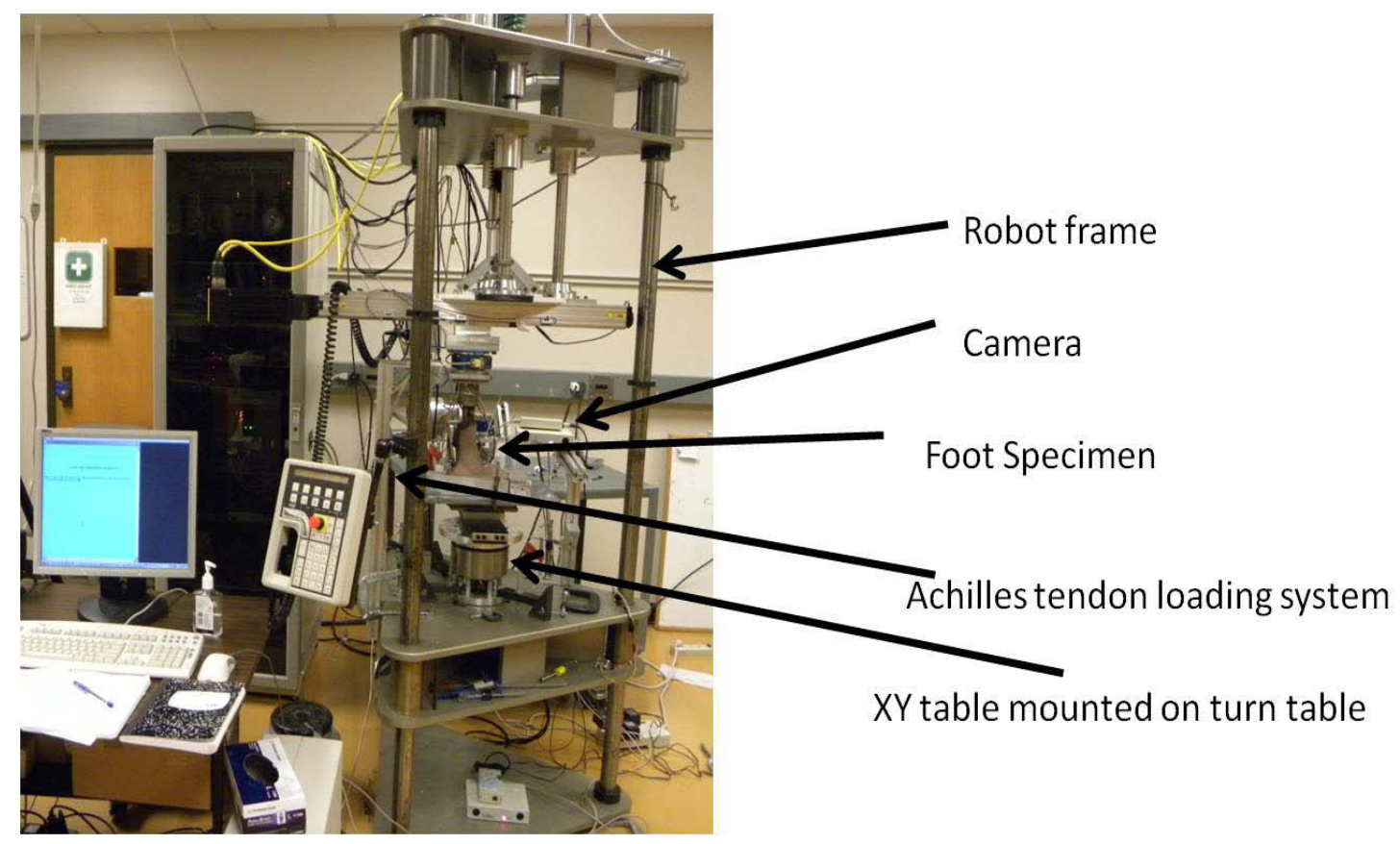

Figure 18: Test set-up (side view) 


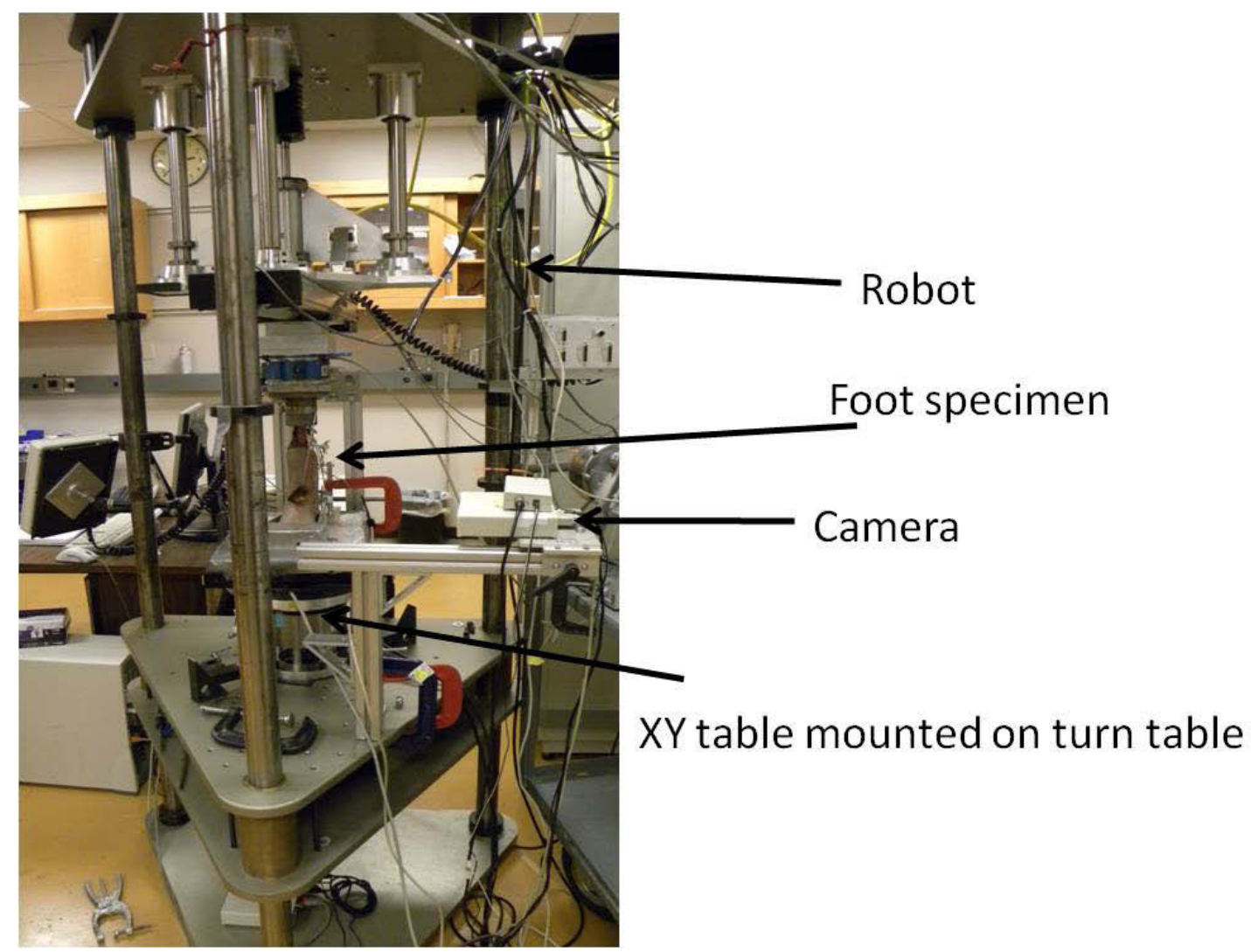

Figure 19: Test set-up (front view) 


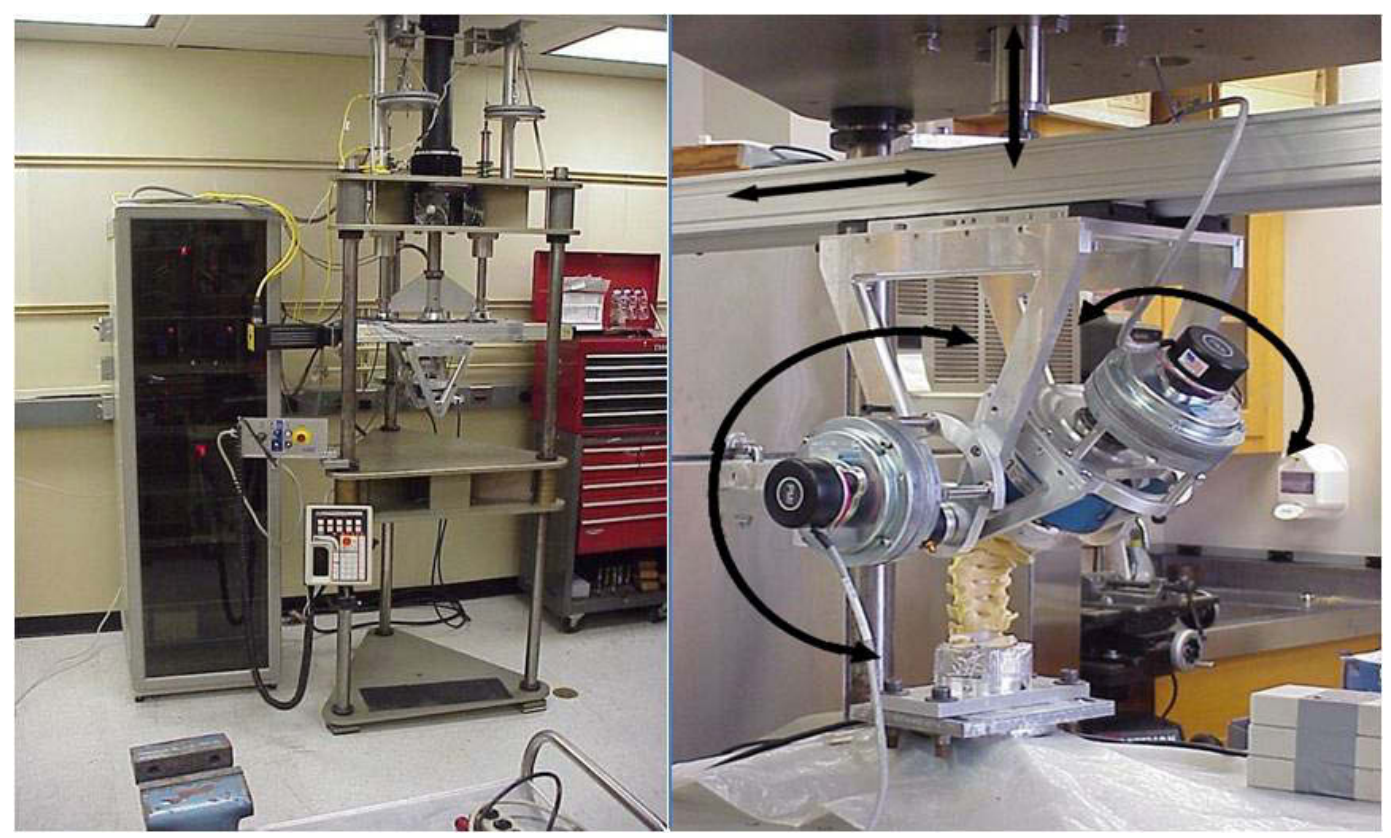

Figure 20: Multiaxis programmable test frame - spine robot (left) four degrees of freedom (right) 


\section{Mounting Fixtures}

Fixtures were designed and machined as required to enable mounting of the foot specimens in the robot. An example of such a fixture is the tibia mounting device, the design of which is explained in detail in Appendix A.

\section{Motion Tracking System}

Standard Kirschner wires (K-wires) were inserted in the specimens as described in the tissue preparation section, on to which, 3D target arrays were mounted. The motion of these 3D targets was tracked by using an optoelectrical camera (Dynasight ${ }^{\mathrm{TM}}$, Origin instruments, Texas). The motion tracking system is seen in Figure 21.

\section{TISSUE PREPARATION}

\section{Selection of Specimens}

Ten matched pairs of feet were procured giving a total of twenty specimens. All specimens were received in a frozen condition. They were thawed in a fridge over a period of 4 days. One specimen out of each pair was assigned to the plate screw group, which would ultimately be fixed using a locked compression plate plus one lag screw, while the other was assigned to the two screws group, which was fixed using two lag screws. A near equal number of right and left feet were assigned to each group with an intention to keep left to right feet ratio close to 1. Each specimen was screened visually as well as by radiographs to determine presence of any obvious deformity. Based on this, three specimens were found defective or having some abnormality. These three specimens and their pairs were eliminated from the study. These specimens, which had deformities, would have required additional surgery to correct the deformities. It was the objective of the study to keep the surgical procedure constant and hence only healthy specimens in which the standard surgical procedure could be performed were selected for this study. Due to this elimination, there was a mismatch in the ratio of right and left feet in each group. There were six right feet and one left foot in two screws group and 6 left feet and 1 right foot in the plate screw group. Thus testing was done on seven matched pairs of specimens (total 14 specimens). During the surgical procedure, one specimen in the plate screw group didn't provide sufficient purchase for the screws and thus had to be removed for the study. Thus the final study consisted of 13 specimens - seven specimens in the two screws group and six specimens in the plate screw group.

\section{Dissection and Potting}

Each specimen was received cut below the knee. The average height of each specimen was between 35 and $45 \mathrm{~cm}$. To achieve a standard height for all specimens, 

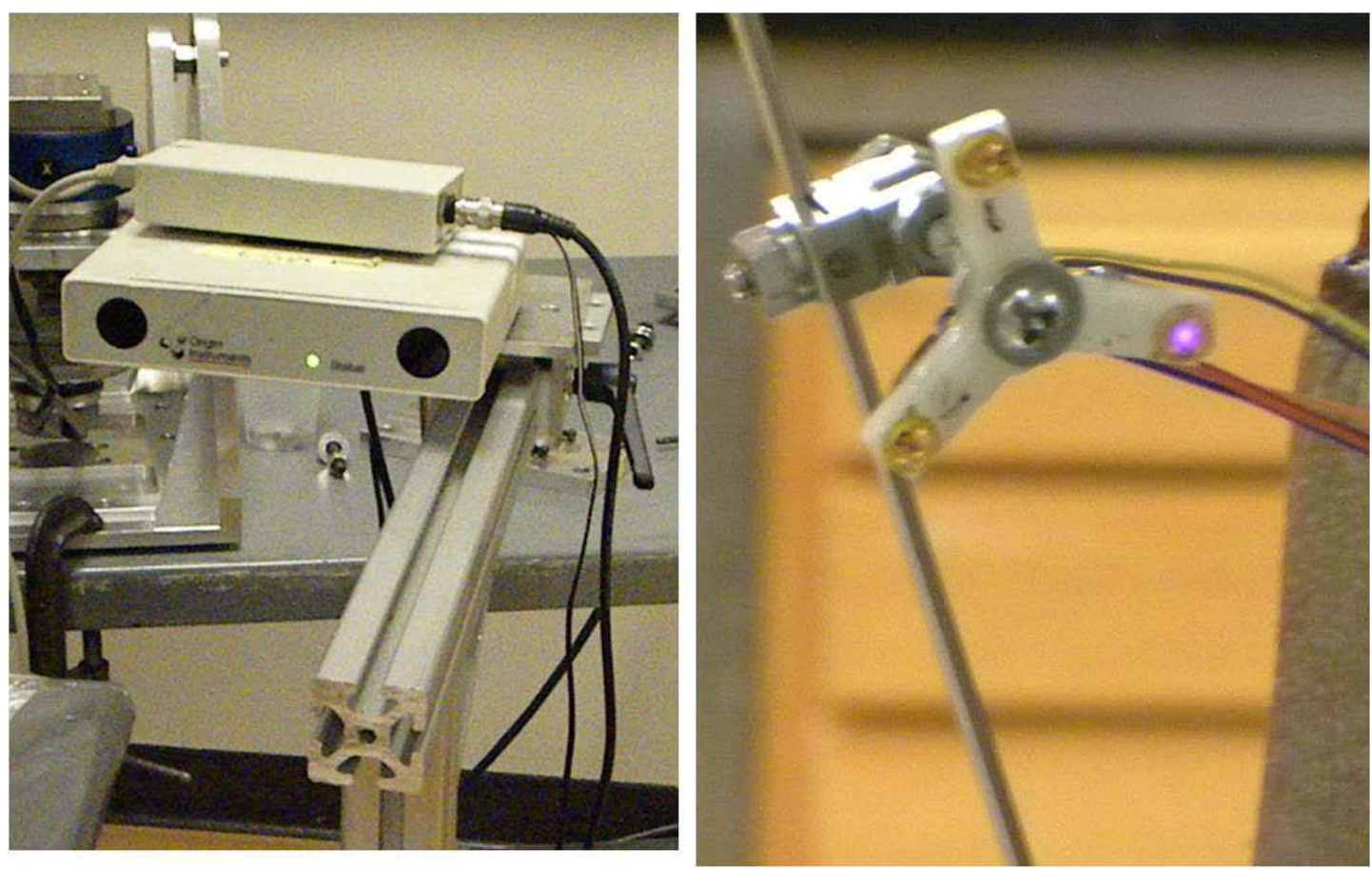

Figure 21: Motion tracking system: Dynasight ${ }^{\mathrm{TM}}$ camera (left) and three dimensional target mounted on a K-wire using clamps (right) 
each specimen was cut at a distance of about 22 to $24 \mathrm{~cm}$ above the lower margin of the medial malleolus. The skin was incised and approximately $10 \mathrm{~cm}$ of skin from the upper cut end was removed. All soft tissues including fat and muscles were dissected and removed till this level. All muscle tissue on the posterior aspect was removed to the bone. Any excess tissue or degenerating skin was removed.

The upper end of the tibia and fibula were made level and upper 3 inches of both bones were stripped clean of all tissue. The surface of the bones was made rough. Holes were drilled into the two bones and wood screws were inserted into the bones. The specimen was subsequently inverted into a custom made cylindrical mold with the upper end of the tibia inside the mold and the tibia hanging vertically downwards while the sole of the foot was horizontal. A low melting point bismuth alloy was melted and poured into the mold and allowed to set. Thus the upper end of tibia was encased in a standardized metal cap which could interface with the fixtures designed for the study.

\section{Achilles Tendon Dissection and K-Wire Insertion for Targets}

The Achilles tendon was conserved throughout this dissection process. The Achilles tendon was stripped off all the muscle tissue. The tendon length was kept to be about $10 \mathrm{~cm}$ above its insertion into the heel bone. The Achilles tendon was fed into a Cable-puller (Figure 22), which could then be attached to the tendon loading mechanism during testing.

Two K-wires were inserted into the foot under fluoroscopic guidance. Of these, one K-wire was inserted into the navicular bone as laterally as possible and other was inserted into talar head or neck as laterally as possible. These K-wires were for mounting of the three dimensional target arrays for tracking using cameras. The $\mathrm{K}$ wires inserted into foot and the targets mounted on the K-wires can be seen in Figure 23.

\section{Storage of Specimens}

Each specimen was wrapped in cotton lined pads after spraying adequately with saline. This prevented drying of the tissue. The specimens were then inserted in plastic bags and labeled appropriately. The wrapped specimens were placed in plastic containers. Each plastic container had a matched pair of feet. The containers were numbered and labeled.

The containers were frozen for storage. At the time of the study, each container was thawed in the refrigerator for 4 days followed by testing or further surgery. At the end of each testing phase, the specimens were replaced inside the containers and frozen. 


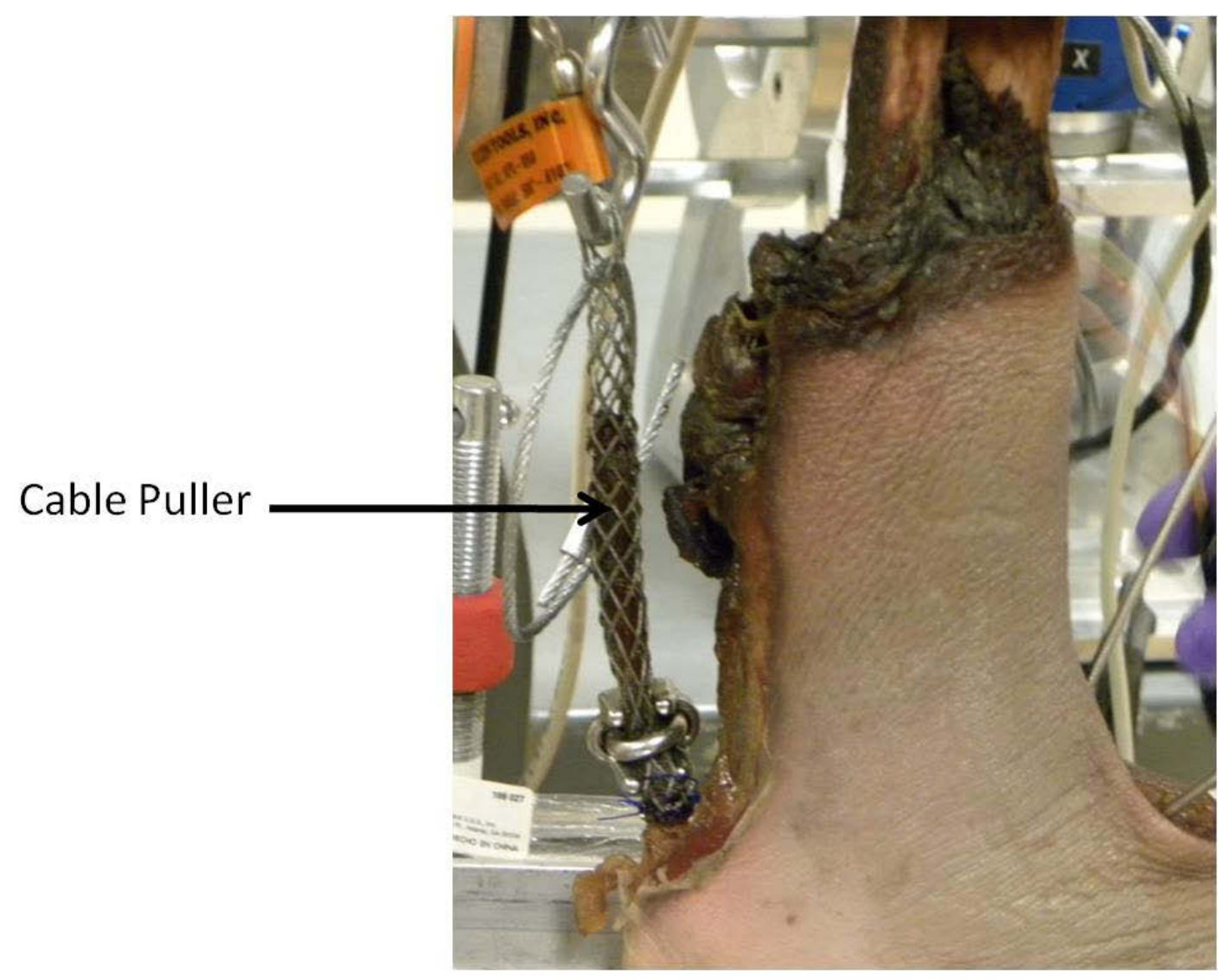

Figure 22: Cable-puller enclosing the Achilles tendon 


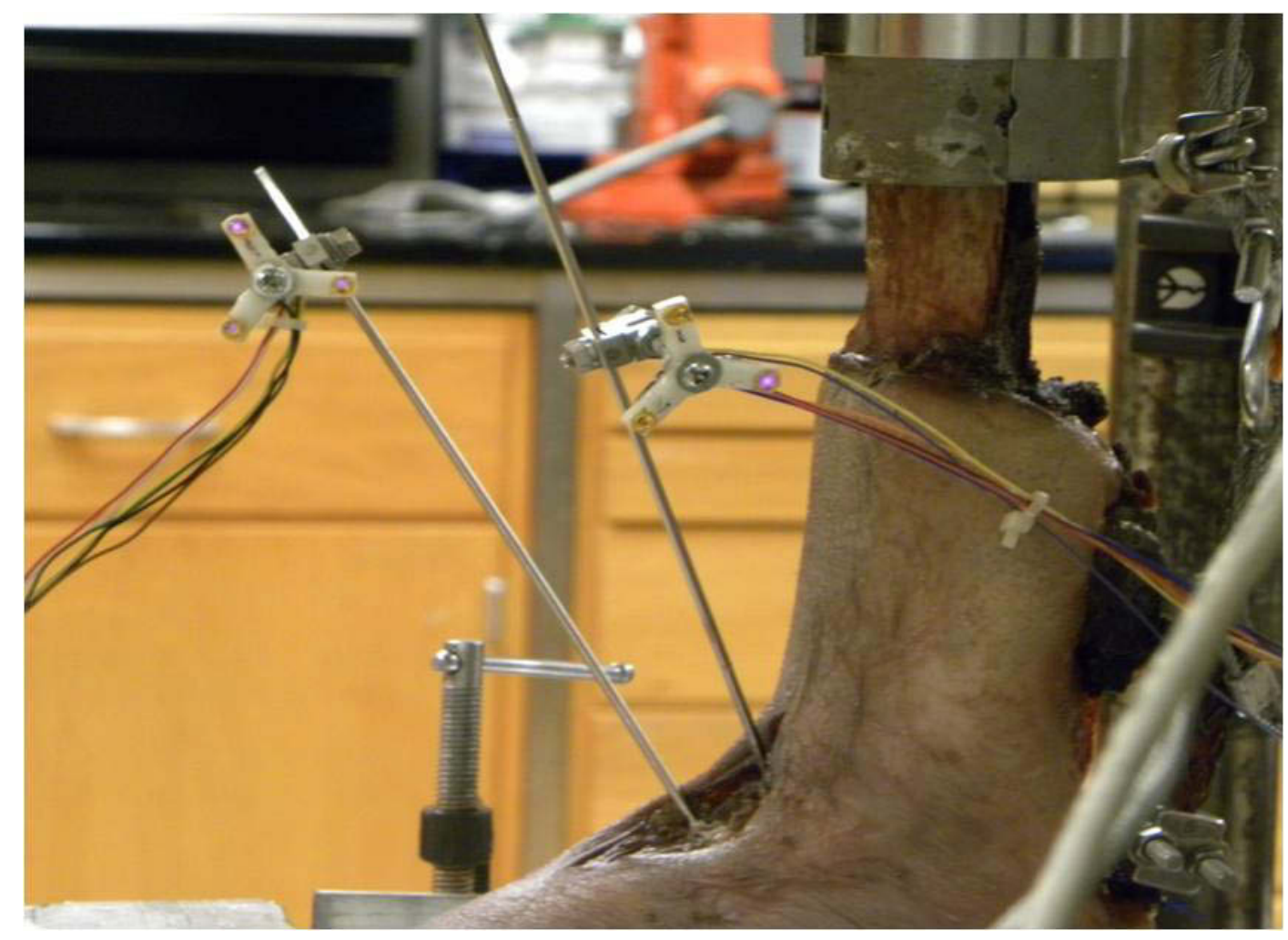

Figure 23: Target arrays mounted on K-wires inserted in bones 


\section{SURGICAL PROCEDURE}

After testing of the specimens in native/harvested state was completed, the specimens were subjected to the following surgical procedure which was similar to the procedure used in clinical cases.

\section{Surgical Exposure}

The initial surgical exposure and joint preparation was standardized for all specimens irrespective of the instrumentation to be used. A standard dorsal-medial approach to the talonavicular joint was performed. The foot was held in the natural position of slight external rotation. A longitudinal skin incision was made on the dorsomedial aspect of the foot superficial to the talonavicular joint. The subcutaneous tissue was dissected. Since this was a cadaveric foot, there was no concern for conservation of nerves or blood vessels. After separation of the subcutaneous tissue, the flexor retinaculum was exposed and cut along the incision. The talonavicular joint could be felt and seen as flaps were developed deeper. The talonavicular joint capsule was incised along the curvature of the navicular and the dorsal and medial surfaces of the talus and navicular bones were debrided to the bone. A $2.5 \mathrm{~mm} \mathrm{~K}$-wire was drilled into each of the bones and the joint cavity was opened by applying a retractor to the K-wires. The joint cartilage was removed using a bone curette and articular surfaces of the bones debrided until the bone was exposed. The natural shape of the bones was conserved. The foot was manipulated into satisfactory alignment, the calcaneus was placed in 5 degrees of valgus and abduction of forefoot was corrected. After this, depending on the type of instrumentation, the specific procedure was performed. The dissection and debridement of the talonavicular joint can be seen in Figures 24 and 25.

\section{Two Screws Technique}

All compression screws were inserted in a retrograde fashion beginning at the naviculocuneiform joint. When two screws were used, the medial screw was inserted at the navicular tuberosity and parallel to the talus. The lateral screw originated in a more dorsal central location aimed in an oblique orientation heading towards the lateral aspect of the talus, but not perforating the lateral gutter. The point of percutaneous insertion for the medial screws is seen in Figure 26. A post-operative radiograph of one of the specimen fixed using two screws is shown as Figure 27.

\section{Locked Plate Plus One Screw Technique}

For the compression screw and plate technique, the medial compression screw was inserted first. The position and orientation of this screw was similar to that described above. A four-hole locking compression plate was used with standardized screw lengths. The plate was placed dorsally on the talonavicular joint with two locked screws on each 


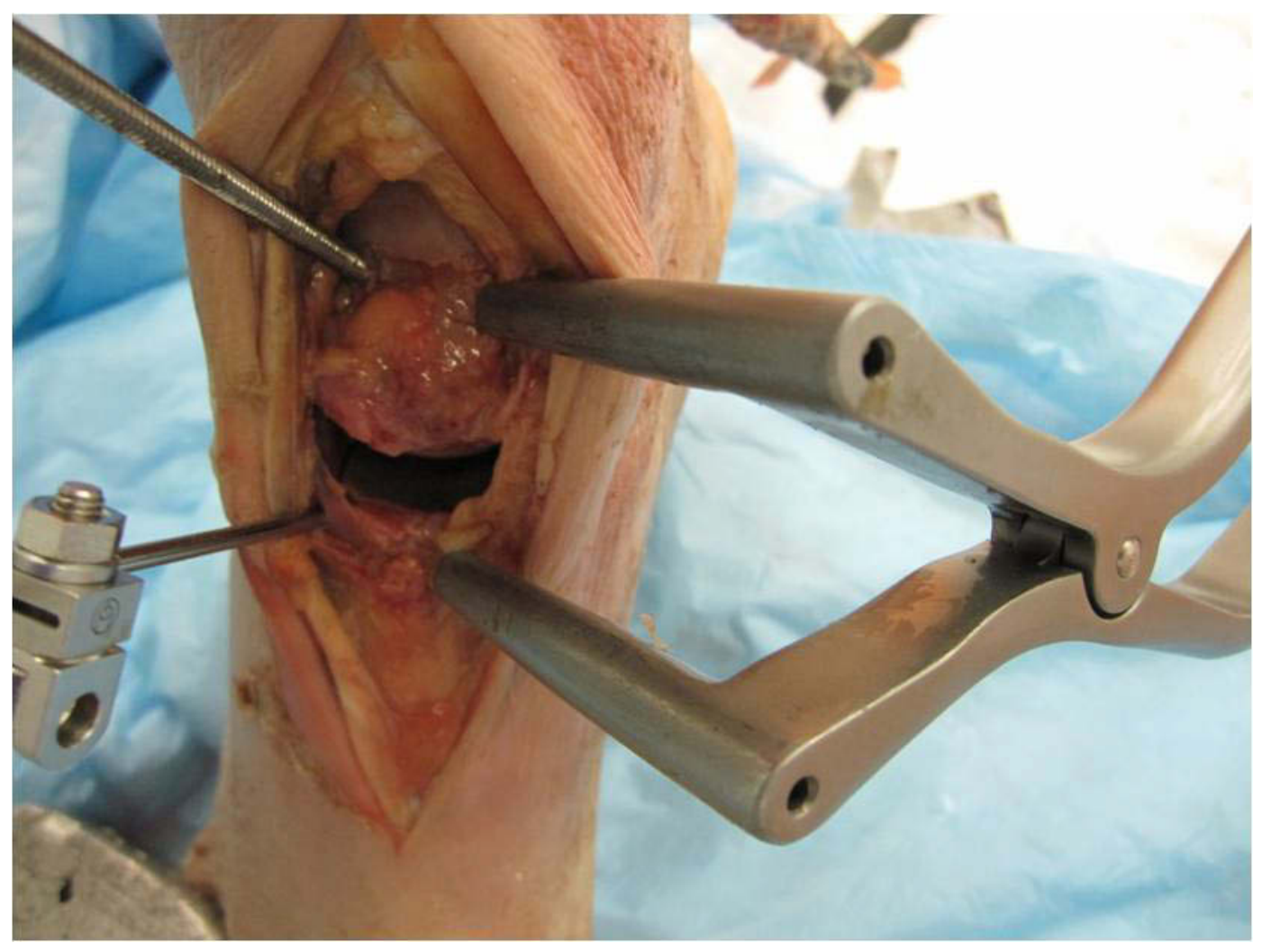

Figure 24: Dissection of the talonavicular joint 


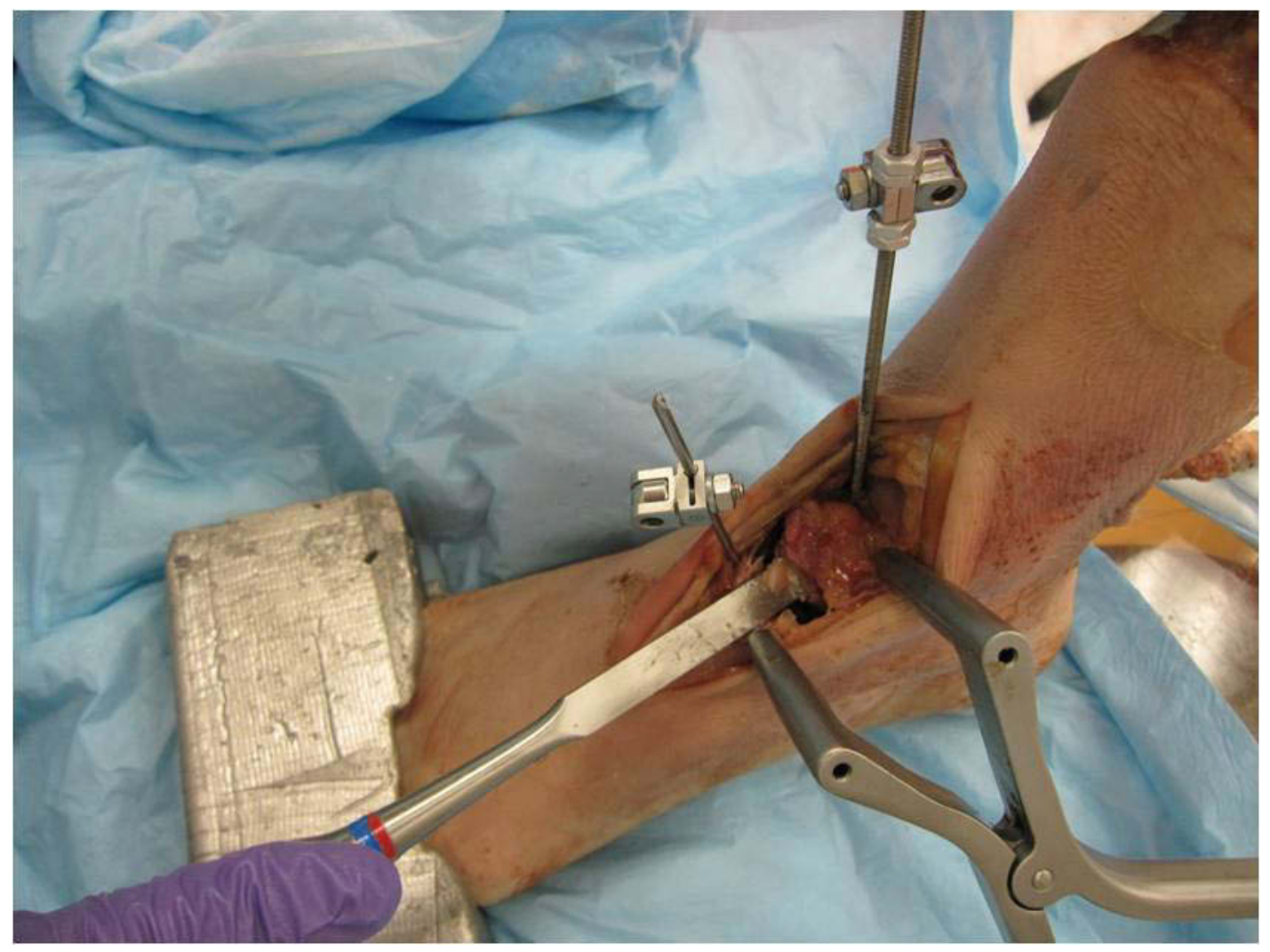

Figure 25: Debridement of the talonavicular joint 


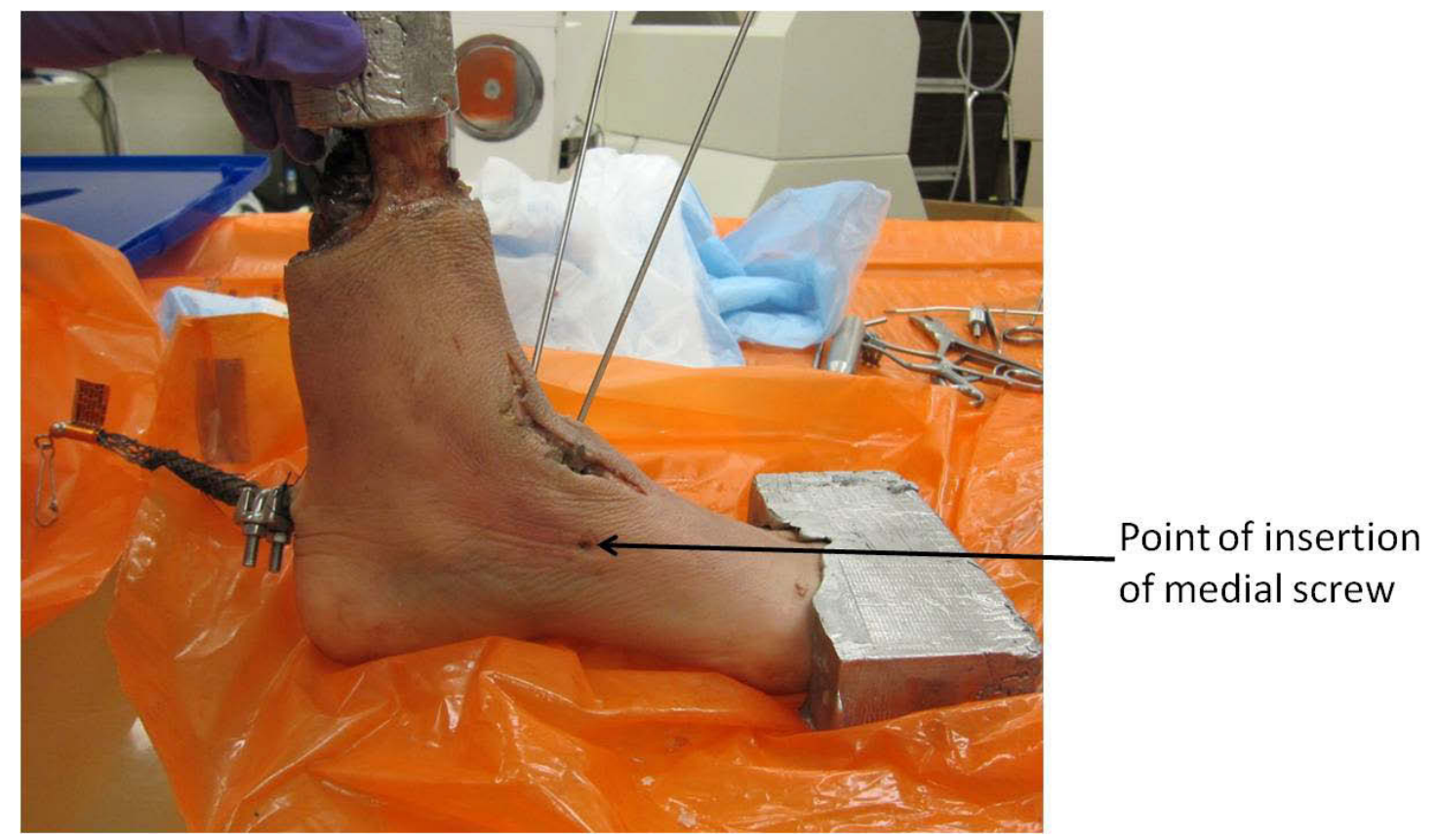

Figure 26: Point of insertion for medial screw 


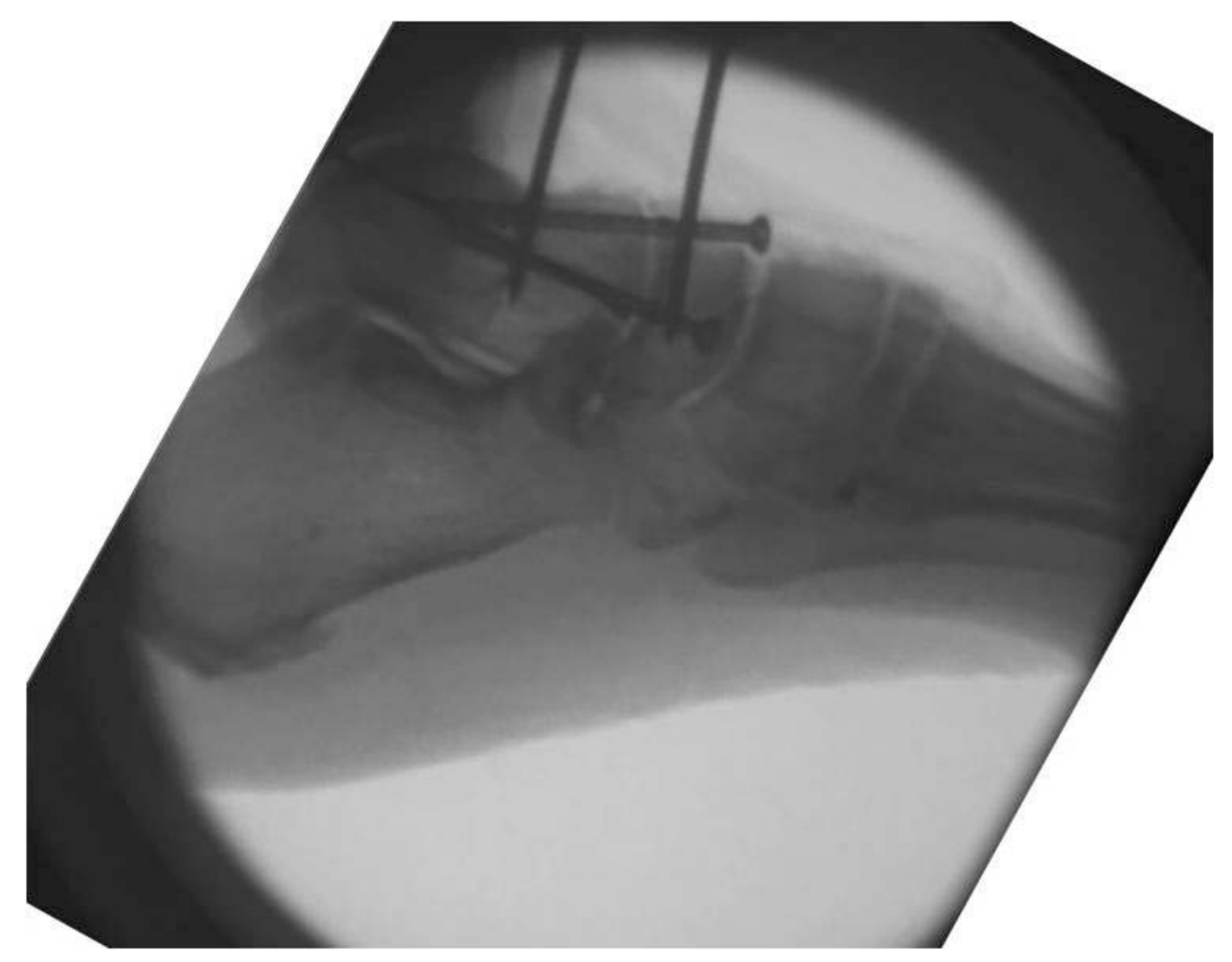

Figure 27: Post-operative radiograph of two screws fixation technique (lateral view)

Note: The radiograph has been rotated. 
side of the joint. After the plate and screw were placed, the lag screw was tightened to maximize compression. At the end of the procedure, the arms of the compression plate were splayed open using the appropriate device. This was to done with an intention of producing further compression. A post-operative photograph (Figure 28) and radiograph (Figure 29) of one of the specimen is shown below.

\section{EXPERIMENTAL METHODOLOGY}

\section{Mounting of Specimens}

The specimens were tested under various loading conditions using a multiaxis programmable test frame. The specimen was prepared as described in the tissue preparation section. The details of the step-by-step procedure used for the experiments are described in Appendix B and a brief summary of the procedure is provided below.

The specimen was mounted on a rectangular base plate which was mounted on a custom designed $\mathrm{XY}$ table. The design and functioning of the $\mathrm{XY}$ table is described in Appendix A. The XY table allowed free motion of the foot specimen in the XY plane. The $\mathrm{XY}$ table was mounted on a programmable turntable. The turn table was used to produce internal and external rotation of the foot.

The upper end of the foot specimen (tibial end) was rigidly attached to the robot using a cylindrical fixture designed for that purpose. The Achilles tendon was loaded using a custom designed loading fixture, description of which is provided in Appendix A. Using this device, the Achilles tendon was loaded using a dead weight of known magnitude. The direction of the loading was near similar to the physiological direction of loading. After mounting the specimen, the foot was tested using four loading scenarios using a program written for that purpose.

The 3D targets were mounted on the K-wires inserted into the specimen and the orientation of the targets was adjusted as required. The motion of these targets was tracked during the tests.

The four loading scenarios were as follows:

1. Internal rotation sequential test

2. Internal rotation simultaneous test

3. External rotation sequential test

4. External rotation simultaneous test 


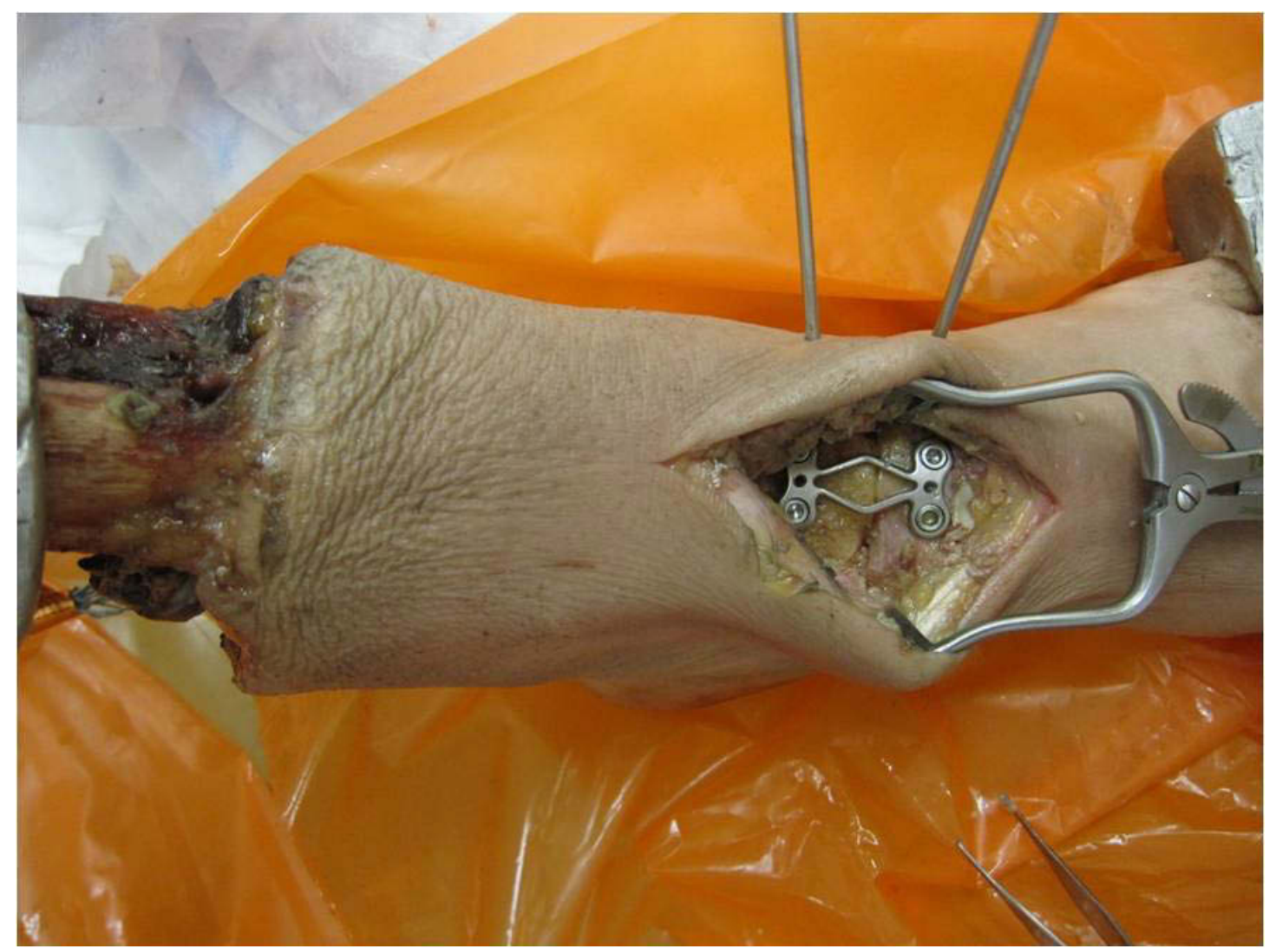

Figure 28: Post-operative photograph of specimen fixed using a locked compression plate plus one screw 


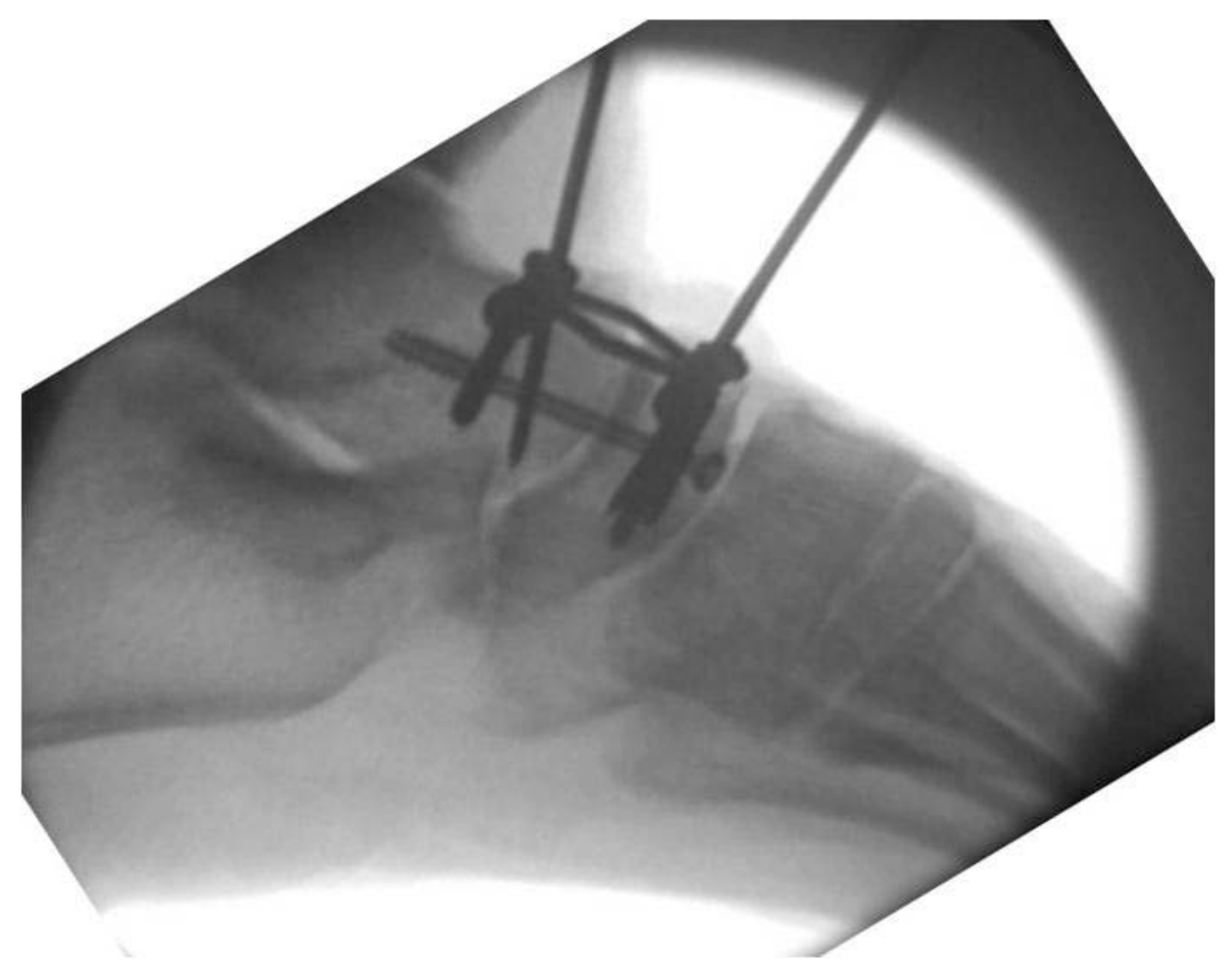

Figure 29: Post-operative radiograph of locked compression plate plus one screw (lateral view)

Note: The radiograph has been rotated. 
All specimens were tested in the native/harvested state and retested using the same loading scenarios after the surgical procedure was performed.

\section{Internal Rotation Sequential Test}

This test (Figure 30) involved application of internal rotation followed by compressive load. The Achilles tendon load of 350N was applied. When this was done, the robot produced a 15 degrees turn using the turn table as long as the moment limit of $10 \mathrm{Nm}$ was not reached. For the internal rotation test, the turn was in clockwise direction for the left feet and counterclockwise direction for the right feet. At the end of rotation, the robot delivered $850 \mathrm{~N}$ of compression to the specimen. The compressive load and the rotation were removed followed by removal of the Achilles tendon load. This test also established the load and moment limits which were used to replay the loading in the internal rotation simultaneous test as described next.

\section{Internal Rotation Simultaneous Test}

This test involved simultaneous application of internal rotation and compressive load. The Achilles tendon load of $350 \mathrm{~N}$ was applied. When this was done, the robot produced a turn using the turn table and simultaneously applied compressive load until the limits as established during the sequential test were achieved. The test terminated automatically if either the load limit or the moment limit were exceeded. For this test, the turn was in clockwise direction for the left feet and counterclockwise direction for the right feet. When the desired load and rotation limits were reached, the compressive load and the rotation were removed followed by removal of the Achilles tendon load.

\section{External Rotation Sequential Test}

This test (Figure 31) involved application of external rotation followed by compressive load. The Achilles tendon load of $350 \mathrm{~N}$ was applied. When this was done, the robot produced a 15 degrees turn using the turn table as long as the moment limit of $10 \mathrm{NM}$ was not reached. For the external rotation test, the turn was in counterclockwise direction for the left feet and clockwise direction for the right feet. At the end of rotation, the robot delivered $850 \mathrm{~N}$ of compression to the specimen. The compressive load and the rotation were removed followed by removal of the Achilles tendon load. This test also established the load and moment limits which were used to replay the loading in the external rotation simultaneous test as described next.

\section{External Rotation Simultaneous Test}

This test involved simultaneous application of external rotation and compressive load. The Achilles tendon load of $350 \mathrm{~N}$ was applied. When this was done, the robot 


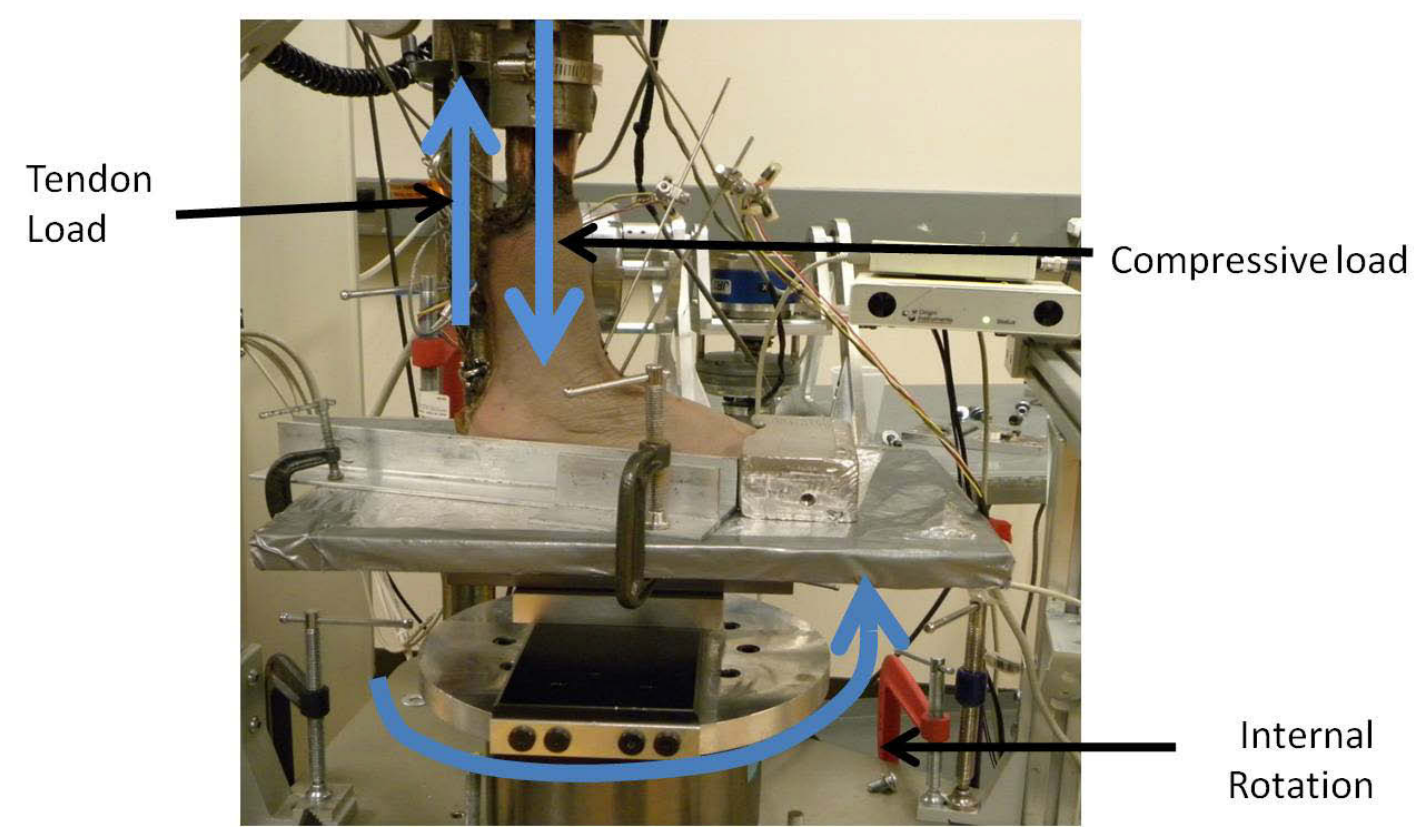

Figure 30: Internal rotation test (for right foot) 


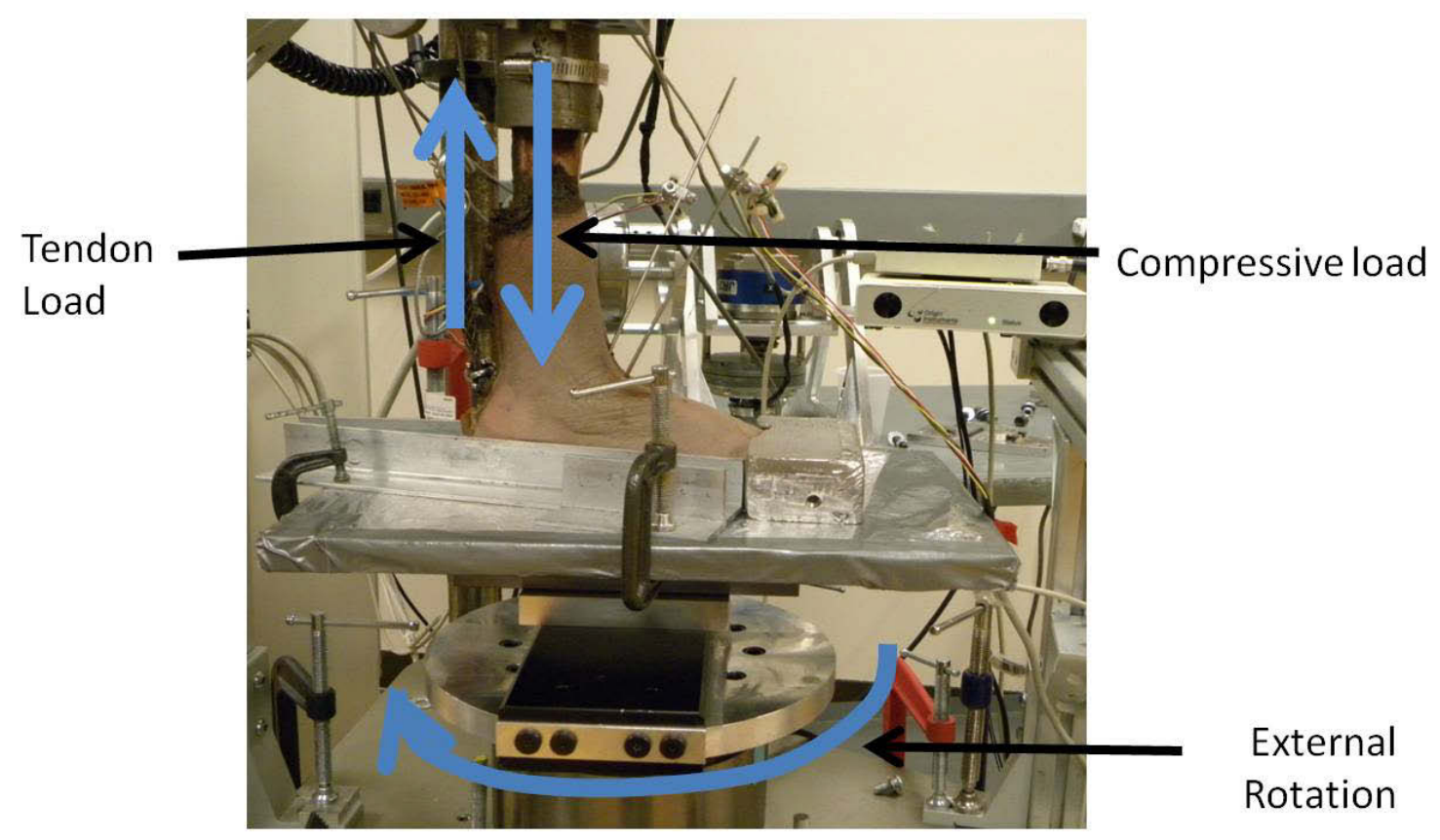

Figure 31: External rotation test (for right foot) 
produced a turn using the turn table and simultaneously applied compressive load until the limits as established during the sequential test were achieved. The test terminated automatically if either the load limit or the moment limit were exceeded. For this test, the turn was in counterclockwise direction for the left feet and clockwise direction for the right feet. When the desired load and rotation limits were reached, the compressive load and the rotation were removed followed by removal of the Achilles tendon load.

\section{DATA RECORDING AND DATA PROCESSING}

During all the tests, the load and the moment values were recorded and saved. These were inspected to ensure that the desired loading limits had been reached. During these tests, the motion of the targets attached to the talus and navicular bones was recorded by using camera and Labview. This provided the XYZ coordinates and the rotations of the targets. The data was saved and processed using the data processing method as described in detail in Appendices C, D, and E. During this process, the position coordinate data of the talus and navicular targets were transformed to a point $\mathrm{J}$ which was located in the joint space.

Point $\mathrm{J}$ was defined as a point located close to the vertex of the convex surface of the Talus which articulated with the Navicular bone. The exact position of point J was defined by using two radiographs - one side view and one top view. The details of the process of determining the location of point $\mathrm{J}$ is described in Appendix C. Figures 32, 33 and 34 illustrate the position of point $\mathrm{J}$.

Once the location of point $\mathrm{J}$ was defined, the offset distances from point $\mathrm{J}$ to the Talus and Navicular Targets were measured. Using this offset data and the instantaneous coordinates of Talus and Navicular Targets, the coordinates of J were obtained. The two sets of coordinates were compared to determine the relative translations and rotations between the two bones which occurred as a result of each of the loading scenarios. The direction of the translation and rotation motion was ignored for the purpose of this study since the purpose of the fixation instrumentation is to prevent motion in any direction.

Once the relative translations and rotations between the two bones in the XYZ camera frame were determined, the relative translations and rotations between the two bones in the joint frame were calculated. The camera frame axes were aligned with the anatomical axes of the body. Thus the XY plane of the camera frame was parallel to the sagittal plane of the human body, the YZ plane was parallel to the coronal plane and the $\mathrm{XZ}$ plane was parallel to the transverse plane of the body. Details of the procedure to define the camera frame and the procedure to determine relative motion in the camera frame are described in the Appendices C, D and E. The validation of this procedure is provided in Appendix F.

The joint frame was determined using measurements from radiographs of specimens and was different for each specimen. In general, the $X^{\prime}$ axis of the joint frame was parallel to the long axis of the talus, the $\mathrm{Y}^{\prime}$ axis of the joint frame was parallel to the 


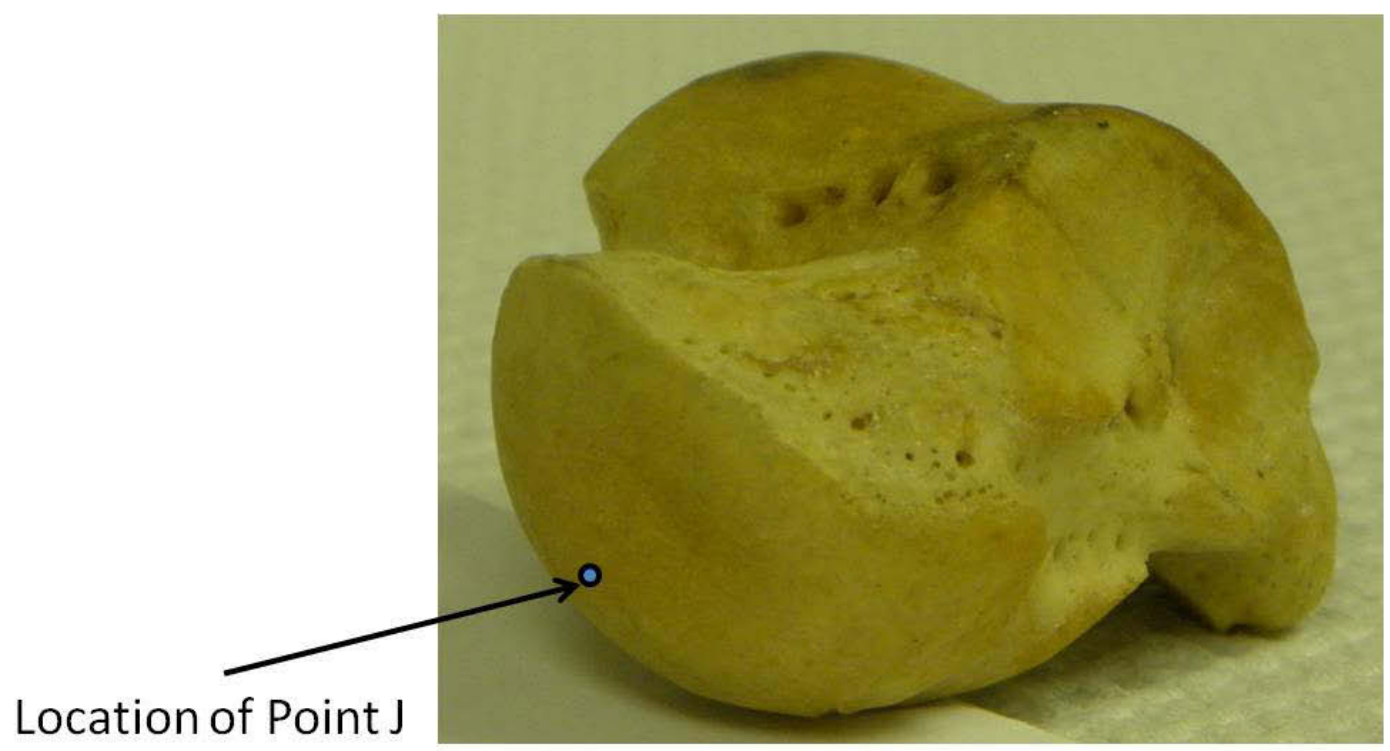

Figure 32: Location of point $\mathbf{J}$ in oblique view 


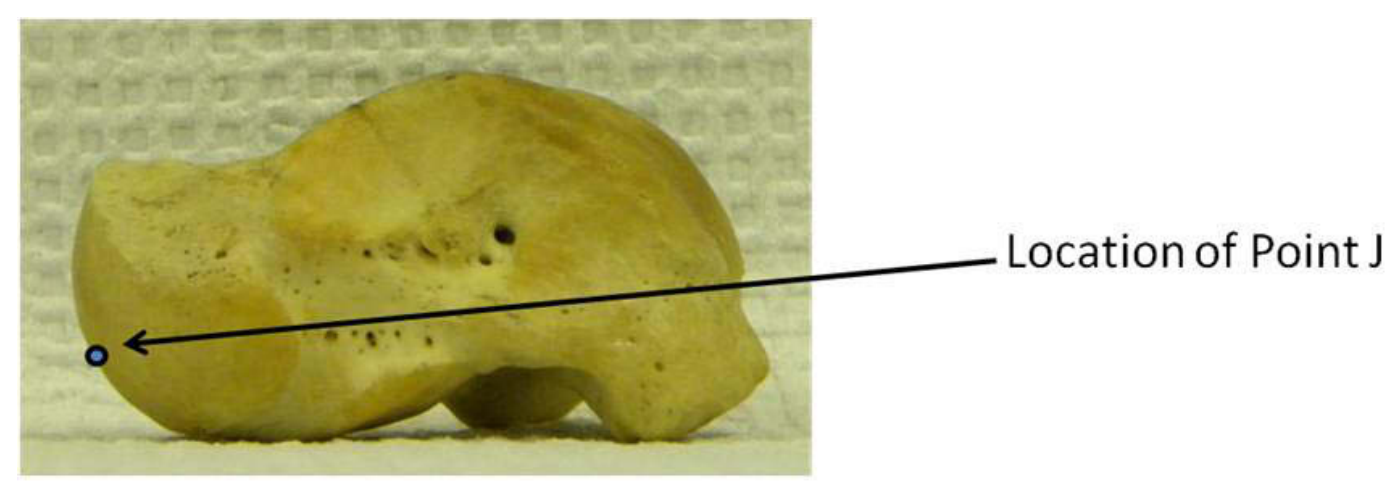

Medial View of Right Talus

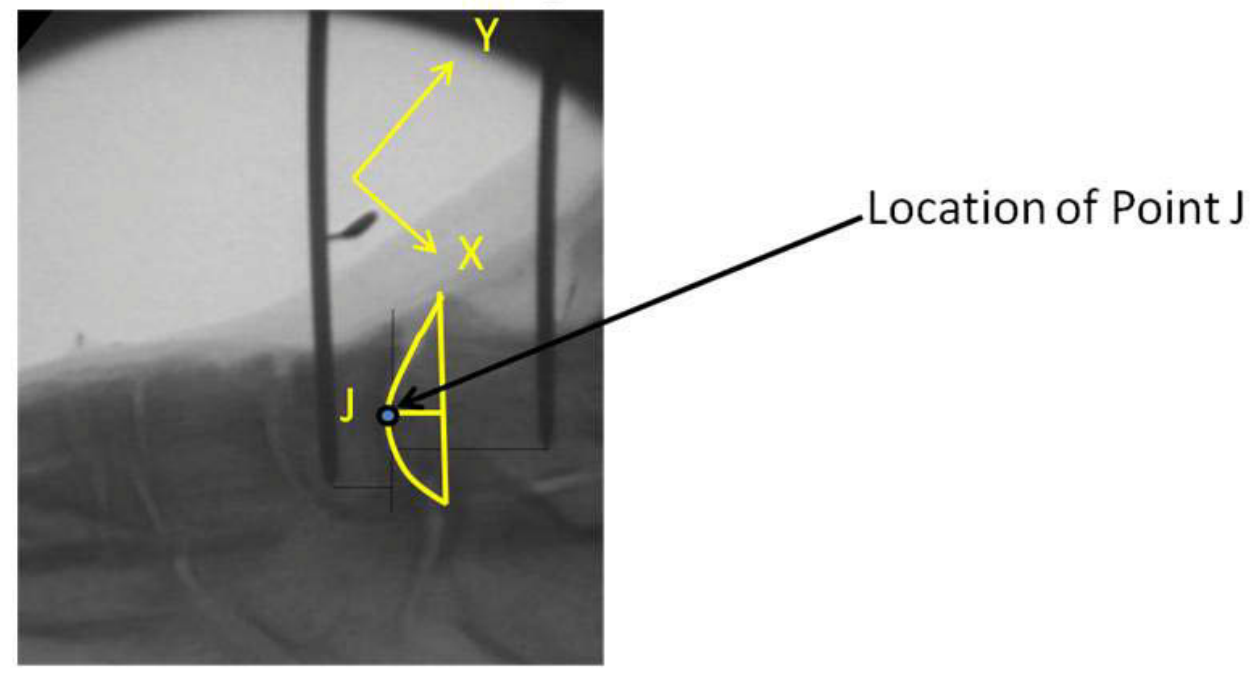

Radiographic View of Right Talus

Figure 33: Location of point $J$ in side view

Note: The radiograph has been rotated. 


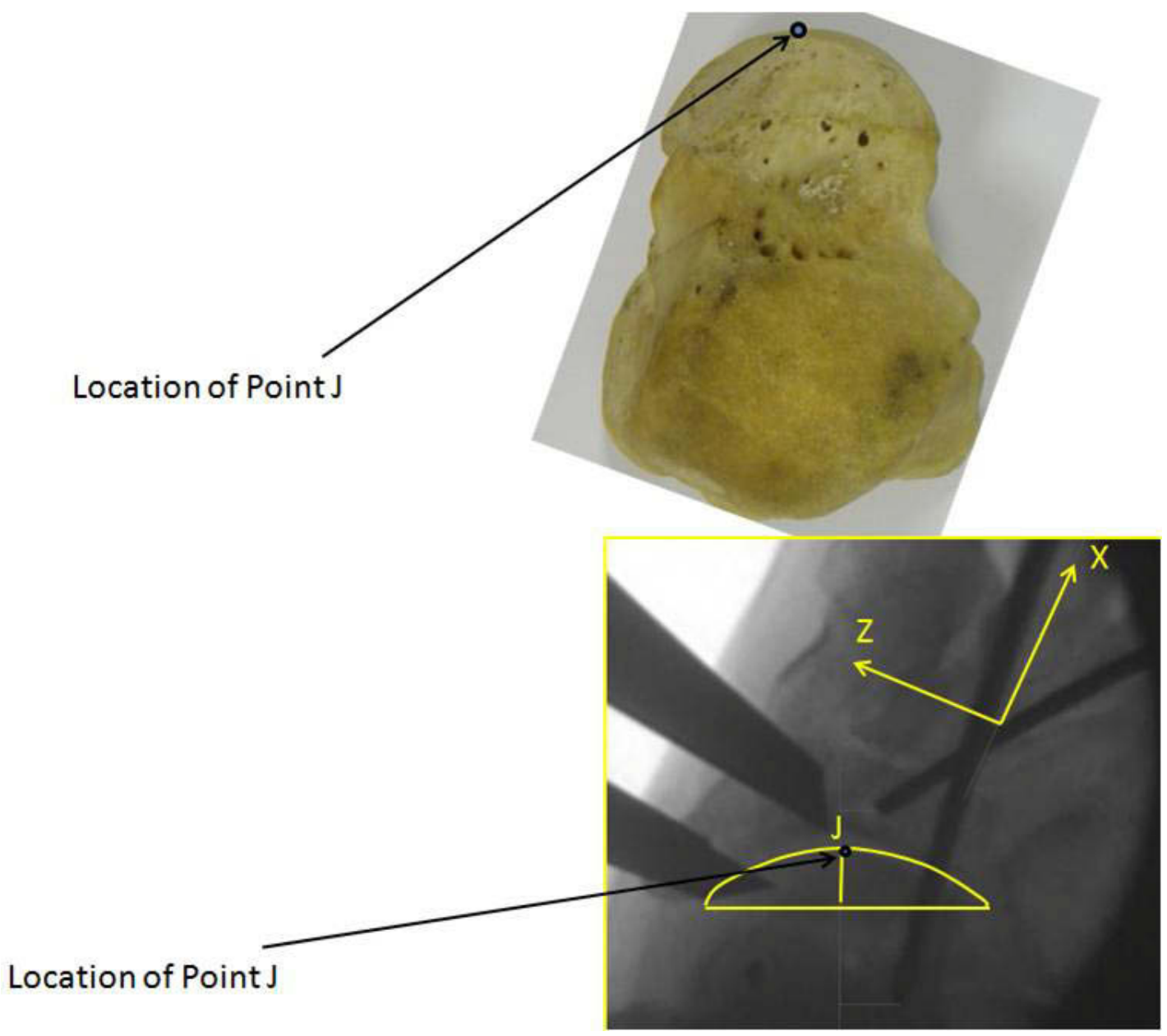

Figure 34: Location of point $J$ in top view

Note: The radiograph has been rotated. 
joint space in the lateral view radiograph and the $Z$ ' axis of the joint frame was parallel to the joint space in the top view radiograph of the specimen.

The details of the measurement process using radiographs and calipers to determine the offset of the talus and navicular targets from the point $\mathrm{J}$ and the process of determining the position of point $\mathrm{J}$ and the orientation of the joint frame are described in Appendix C. The detailed procedure of finding the separation between the bones in the camera frame and the joint frame are described in Appendix E. The camera frame and the joint frame are depicted in the figures in the next section.

\section{DATA MANAGEMENT AND STATISTICS}

At the end of the above process, for each of the loading scenarios, the separations of the bones along the $\mathrm{X}, \mathrm{Y}$ and $\mathrm{Z}$ axes of the camera frame and along the $\mathrm{X}^{\prime}, \mathrm{Y}^{\prime}$ and $\mathrm{Z}^{\prime}$ axes of the joint frame were obtained. Also the relative rotations between the bones about $\mathrm{X}, \mathrm{Y}$ and $\mathrm{Z}$ axes of the camera and about the $\mathrm{X}^{\prime}, \mathrm{Y}^{\prime}$ and $\mathrm{Z}^{\prime}$ axes of the joint frame were obtained. The orientation of the axes of the joint frame in relation to the camera frame is depicted in Figures 35 and 36.

Since the objective of the surgery and instrumentation was to achieve fixation, any instrumentation which shows less motion as compared to the other can be considered to be better or more effective.

The results were compared for statistically significant difference in motion. Due to the complex nature of the data, a Three factor ANOVA with a mixed model had to be used. The statistical analysis was done by Elizabeth Tolley, Ph.D. using mixed procedure of SAS software version 9.1.3.

The three factors were:

1. State of the specimen: Harvested or Instrumented

2. Treatment received: Screws or Plate

3. Loading conditions: 4 types of loading conditions

For translation data, the design for statistical analysis was a split plot with specimens nested within treatments and conditions and states cross-classified with treatments and specimens. Only the X, Y, Z dimensions were analyzed separately. The specimens were nested within treatments since each specimen received either - a plate plus one screw or two screws but not both. The model took into account inter-specimen variation and inter-group variations. Thus it was possible to compare the instrumented specimen groups with the harvested groups to find the efficacy of the two fixation techniques in reducing translation. 


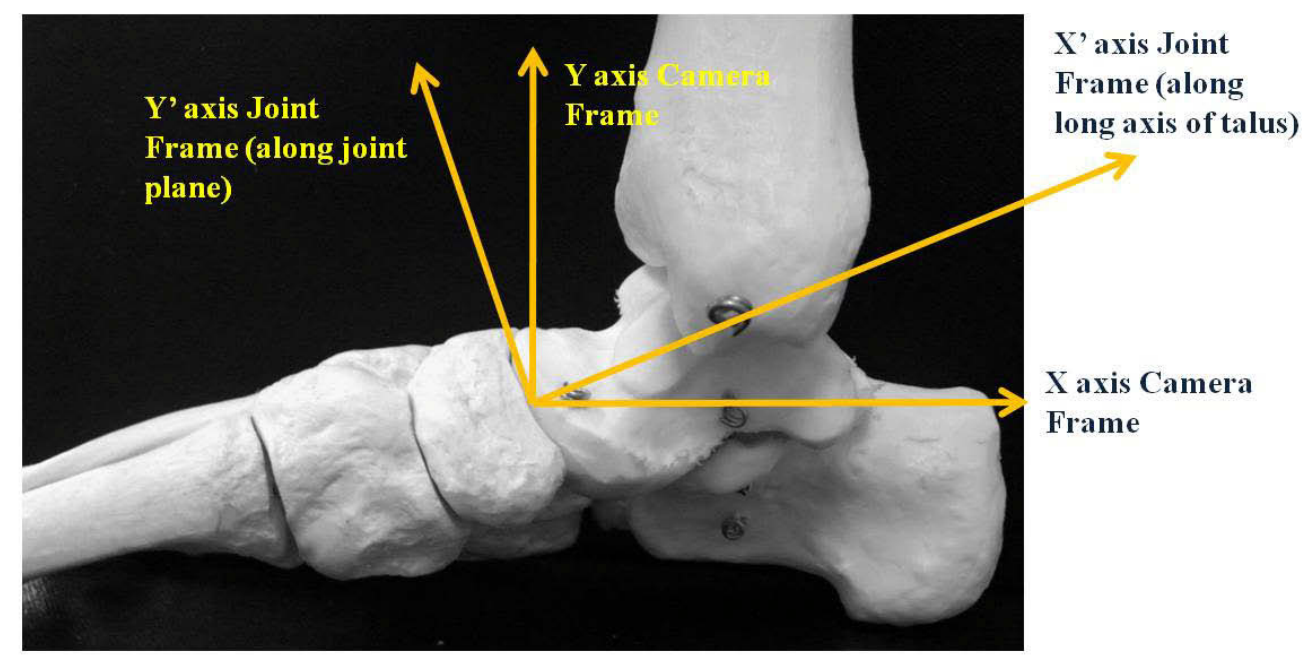

\section{Camera}

Figure 35: $\quad$ Reference frames (medial view) 


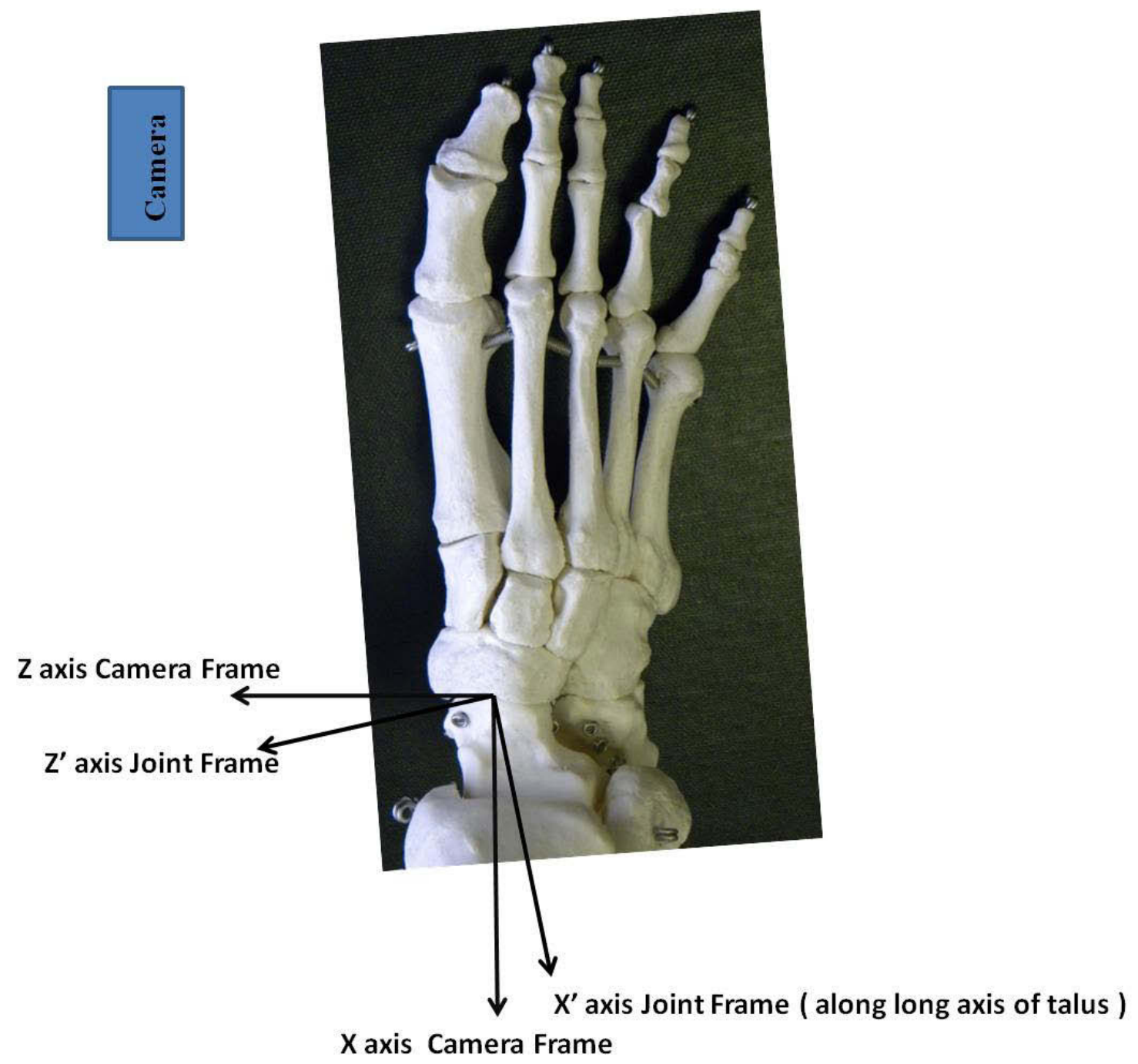

Figure 36: Reference frames (top view) 
For rotation data the design for statistical analysis was again split plot with specimens nested within treatments and conditions and states cross-classified with treatments and specimens. But the $\mathrm{X}, \mathrm{Y}, \mathrm{Z}$ dimensions and the states were analyzed separately. It was observed that there was a large difference in variances associated with the two states for rotational motion. These differences violate the assumptions necessary for ANOVA. Also some of the data were for particular dimensions extremely skewed to the right for the rotation data. Hence the statistical model had to be changed and the states had to be analyzed separately.

The results were analyzed in both the camera frame and the joint frame. It was possible to observe the difference between the results obtained in the two frames and thus the advantage of analyzing the results in the local joint frame was demonstrated. 


\section{CHAPTER 5: RESULTS}

The relative translation and rotations between the bones in the harvested/native state and instrumented states under each of the loading conditions were obtained. The motion data was processed in both camera frame (CF) and joint frame (JF) and the results of the groups compared for statistically significant difference in both the camera frame and the joint frame.

The translation and rotation data for each specimen along with the means and the standard deviations are presented in the tables in Appendix G. Tables 1 to 6 provide the relative translation in the camera frame, Tables 7 to 12 provide the relative rotations in the camera frame, Tables 13 to 18 provide the relative translation in the joint frame, and Tables 19 to 24 provide the relative rotations in the joint frame. These results are depicted in figures in this chapter.

Each graph in this chapter, depicts the translation or rotation with respect to one of the three mutually perpendicular axes i.e., $\mathrm{X}, \mathrm{Y}, \mathrm{Z}$ axes for the camera frame and $\mathrm{X}^{\prime}$, $Y^{\prime}, Z^{\prime}$ axes for the joint frame. Each graph shows four sets of data corresponding to the four loading conditions, i.e., internal rotation sequential test, internal rotation simultaneous test, external rotation sequential test, and external rotation simultaneous test. In the graphs these sets are named as IRCSe, IRCSi, ERCSe, and ERCSi respectively. In each sets are four bars corresponding to (from left to right) harvested data for two screws group, harvested data for plate screw group, post-surgical i.e. instrumented data for two screws group and instrumented data for plate screw group respectively. For the translation graphs, the vertical axis represents relative translation in millimeters. For the rotation graphs, the vertical axis represents relative rotation in degrees. In all the graphs, the error bars represent one standard deviation from mean.

\section{CAMERA FRAME}

As seen in Figures 35 and 36, and described in previous chapter, the camera frame is aligned with the axes of the camera and thus aligned with the anatomical axes of the body. The relative translation and rotations between the talus and navicular bones was determined. The relative translations and rotations between the bones in the camera frame are depicted in Figures 37 to 42 . The results were compared for statistical difference using SAS as described earlier. The results of the statistical tests are described below along with the graphs.

Figures 37 to 39 show the relative translations between the talus and navicular bone along each axis of the camera frame. In each of the three graphs, the vertical axis represents relative translation in millimeters. Statistical tests on translation results for $\mathrm{X}$, $\mathrm{Y}$ and $\mathrm{Z}$ axes of camera frame reported no significant differences between any of the groups. 


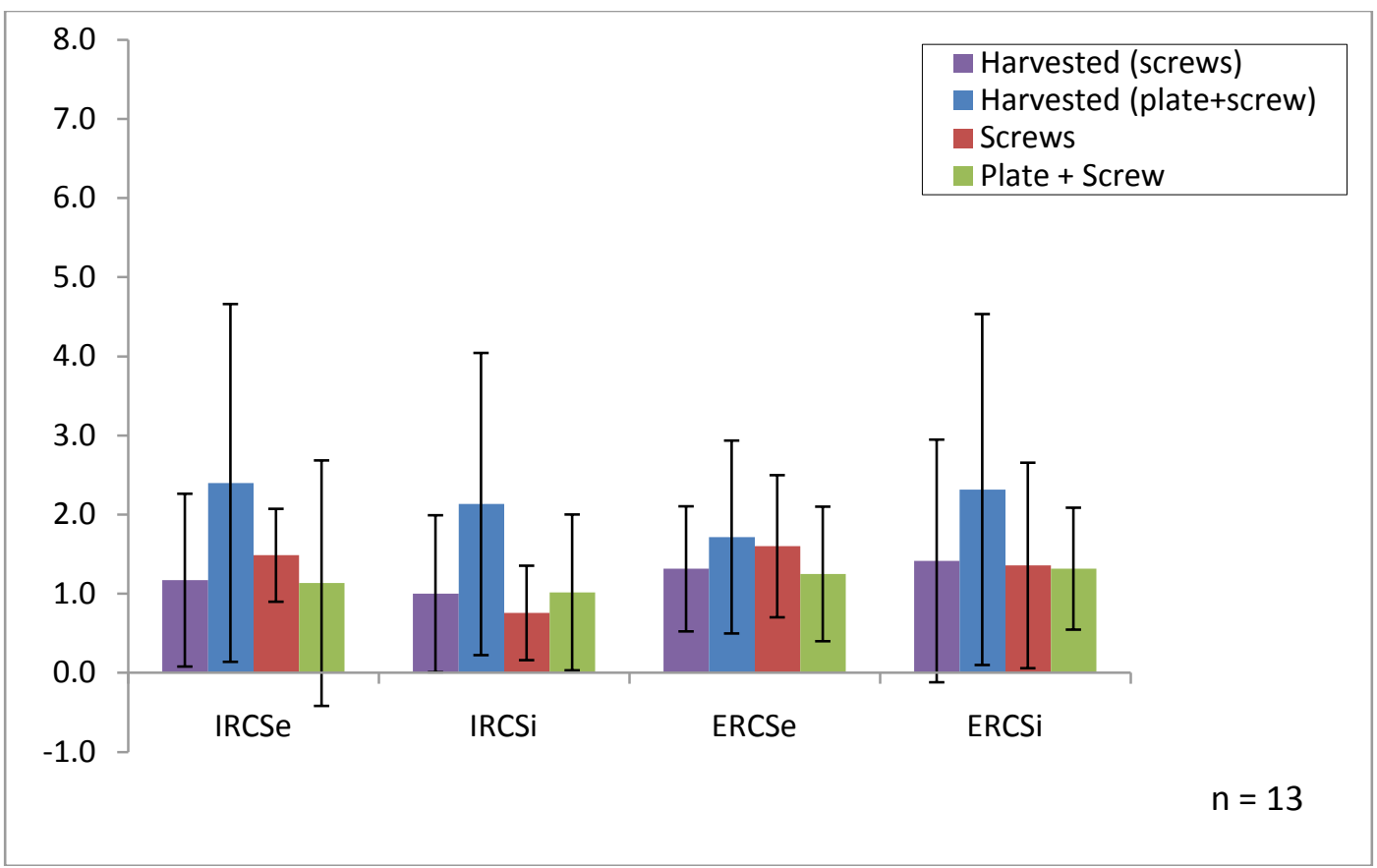

Figure 37: Relative translation along $X$ axis (camera frame) 


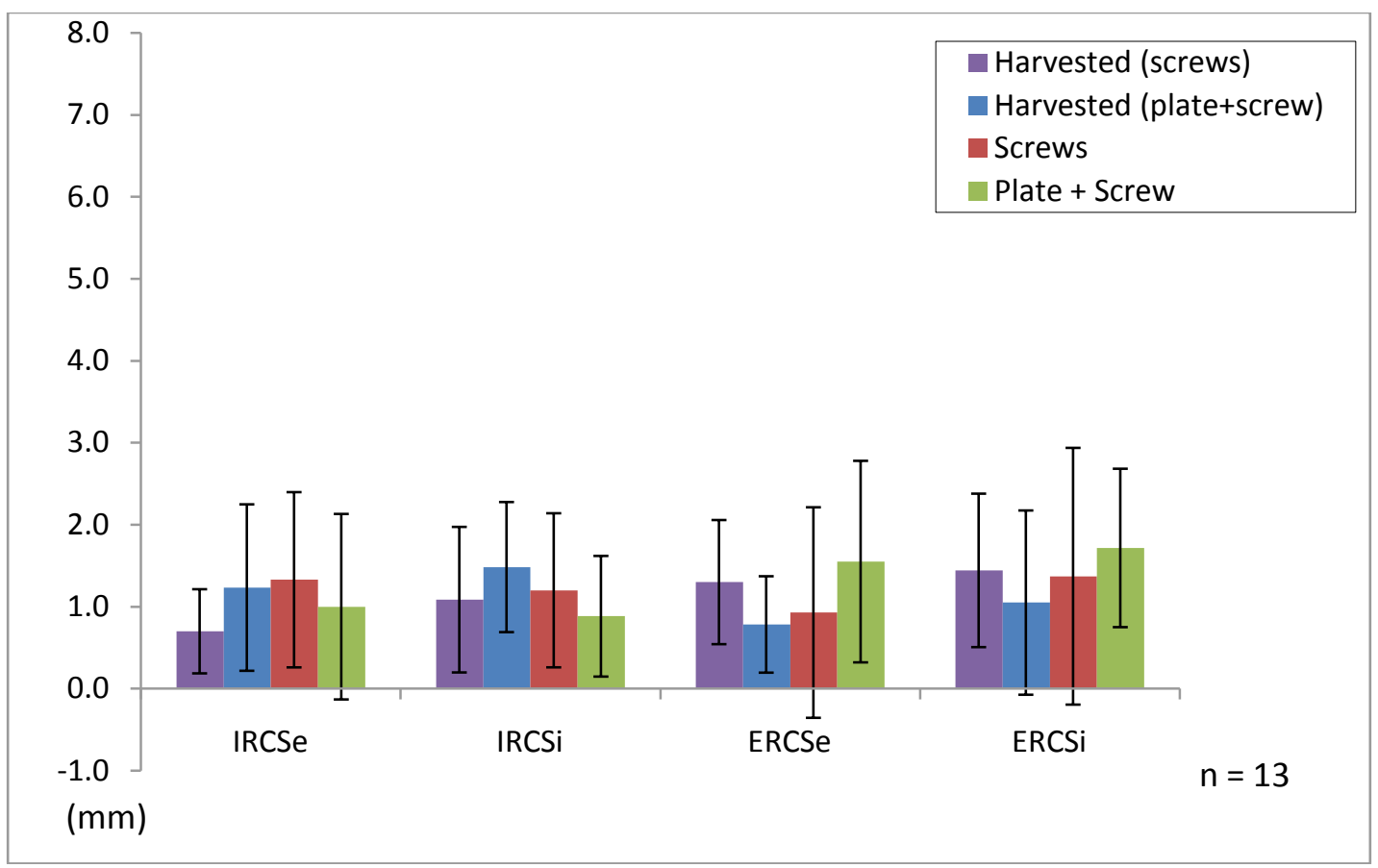

Figure 38: $\quad$ Relative translation along $Y$ axis (camera frame) 


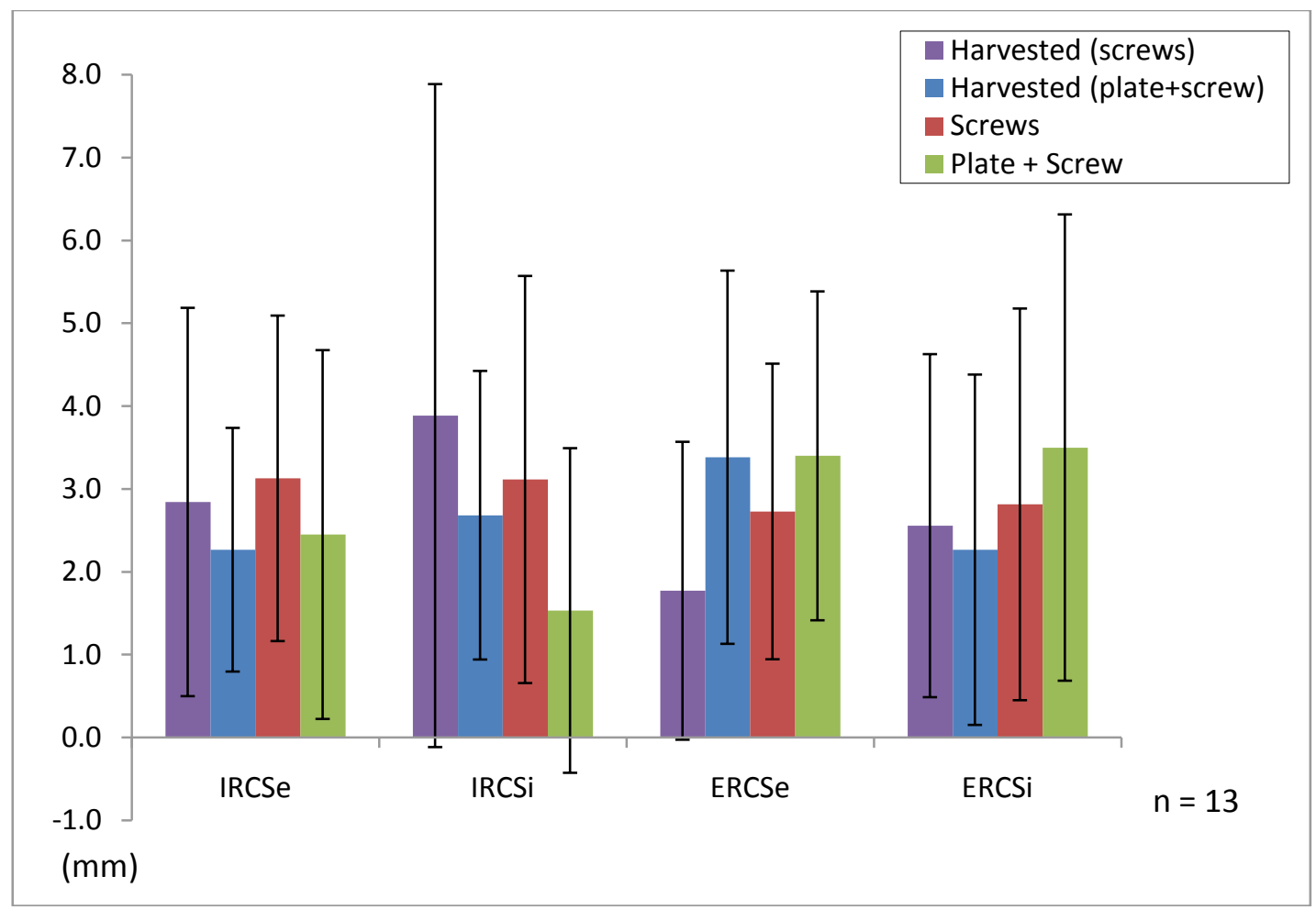

Figure 39: Relative translation along $\mathrm{Z}$ axis (camera frame) 


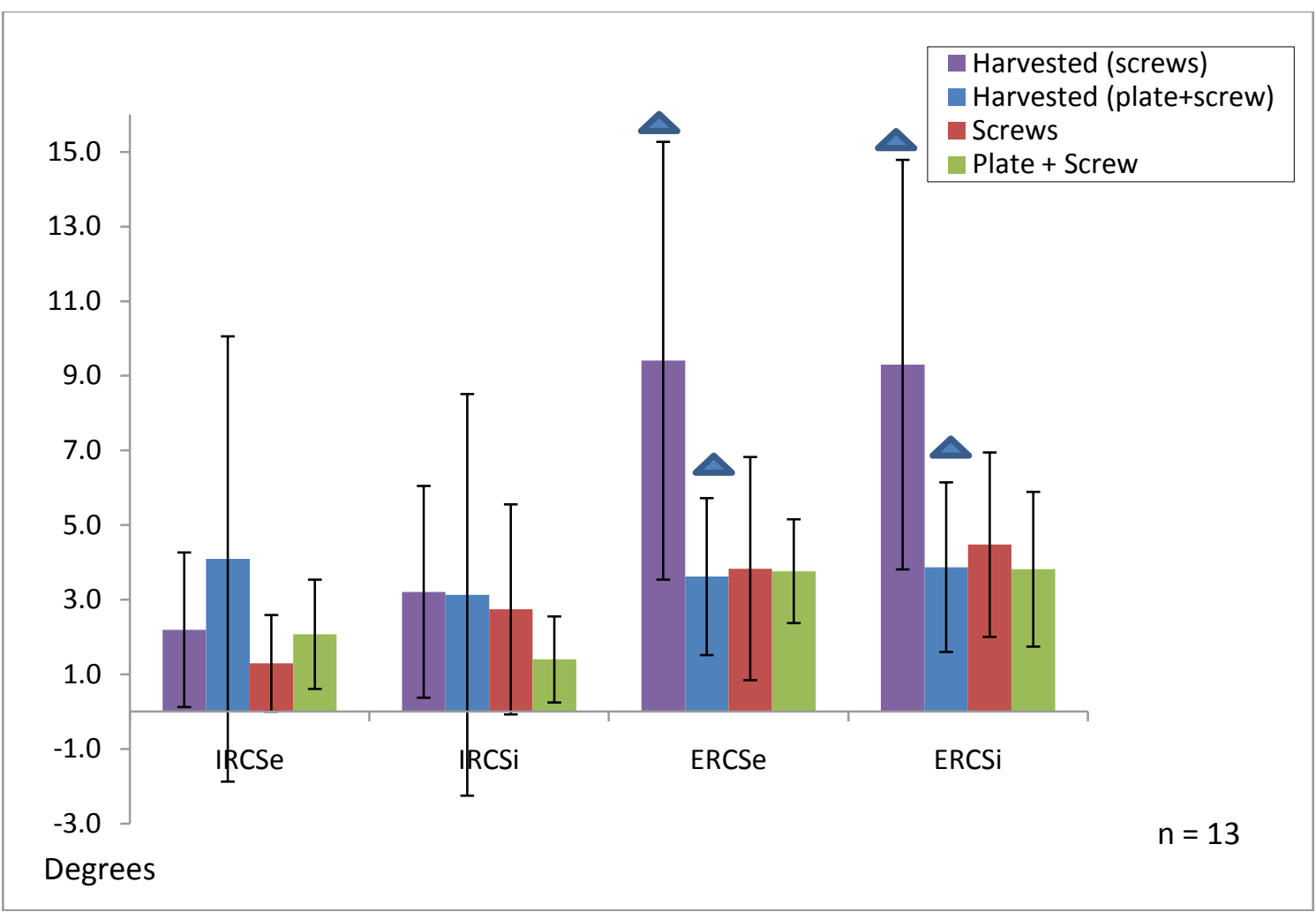

Figure 40: Relative rotation about $\mathrm{X}$ axis (camera frame)

Note: $\Delta \quad=\quad$ Significant difference between pre-operative groups. 


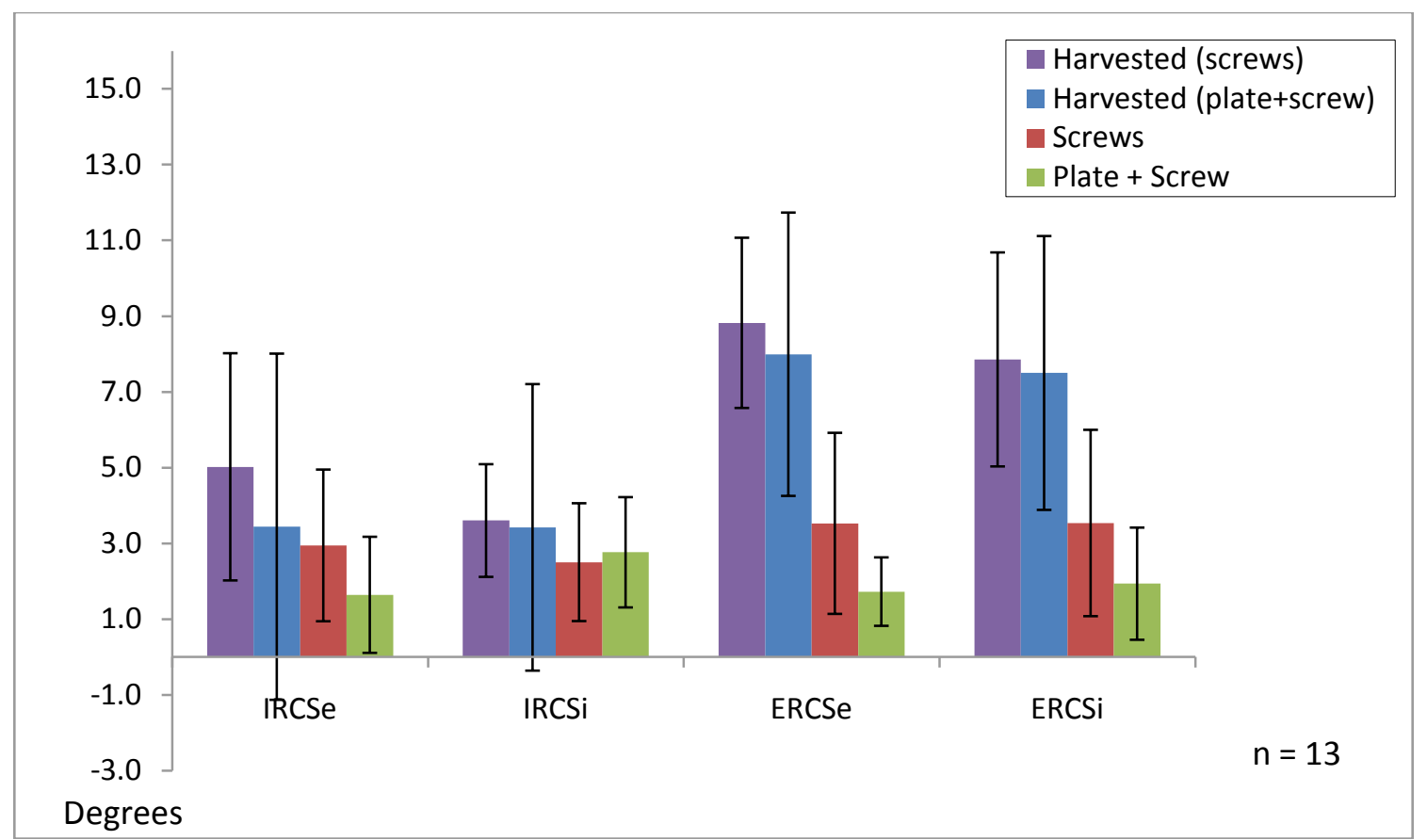

Figure 41: Relative rotation about $Y$ axis (camera frame) 


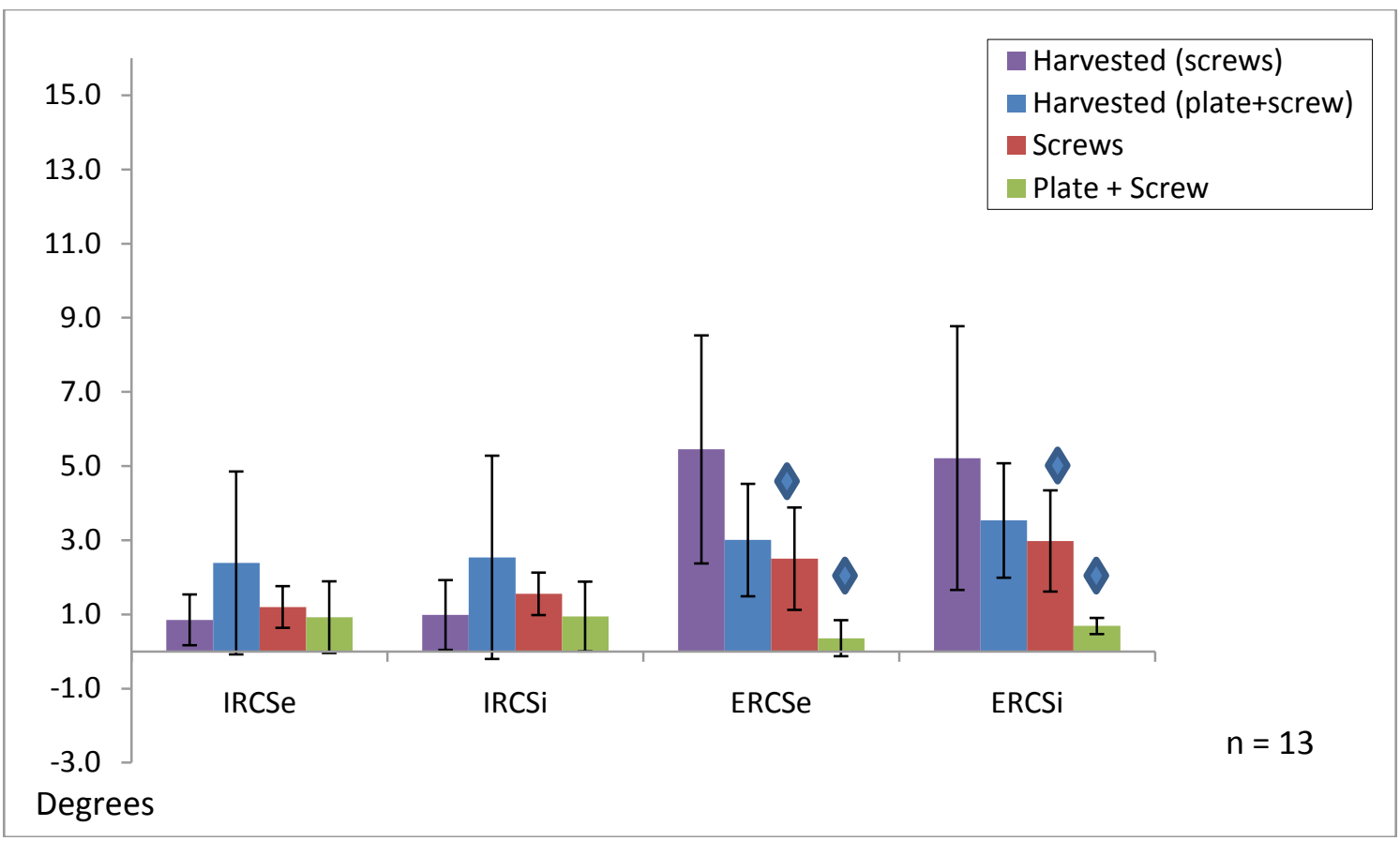

Figure 42: $\quad$ Relative rotation about $Z$ axis (camera frame)

Note: $\quad=\quad$ Significant difference between post-operative groups. 
The mean translation along $\mathrm{X}$ axis was between $1 \mathrm{~mm}$ and $2.4 \mathrm{~mm}$ preoperatively and between $0.8 \mathrm{~mm}$ and $1.6 \mathrm{~mm}$ post-operatively. The mean translation along $\mathrm{Y}$ axis was between $0.7 \mathrm{~mm}$ to $1.5 \mathrm{~mm}$ pre-operatively and between $0.9 \mathrm{~mm}$ and $1.7 \mathrm{~mm}$ post-operatively. The mean translation along $\mathrm{Z}$ axis was between $1.8 \mathrm{~mm}$ and $3.9 \mathrm{~mm}$ pre-operatively and between $1.5 \mathrm{~mm}$ and $3.5 \mathrm{~mm}$ post-operatively.

Figures 40 to 42 show the relative rotations between the talus and navicular bone about each axis of the camera frame. In each of the three graphs, the vertical axis represents relative rotation in degrees. The rotations about $\mathrm{X}, \mathrm{Y}$ and $\mathrm{Z}$ axes of camera frame were compared for statically significant difference. There was a statistically significant difference in rotations about $\mathrm{X}$ axis between the plate screw group and the two screws group pre-operatively, during external rotation sequential and external rotation simultaneous tests. The $\mathrm{P}$ values were 0.02 and 0.03 respectively. The two screws group was showing significantly more rotation as compared to plate screw group.

Post-surgically there was a highly significant difference between the plate screw group and two screws group in rotation about $\mathrm{Z}$ axis during external rotation sequential and external rotation simultaneous tests with $P$ values of 0.0002 and less than 0.0001 . The plate screw group was showing significantly less rotation in these cases. No other rotation result showed statistically significant difference.

\section{JOINT FRAME}

As seen in Figures 35 and 36, and described in previously, the joint frame is aligned with the axes of the articulating bones and thus aligned with the joint plane. Thus the $\mathrm{X}^{\prime}$ axis is parallel to the long axis of the Talus, and the $\mathrm{Y}^{\prime} \mathrm{Z}^{\prime}$ plane is parallel to the true joint plane. The relative translations and rotations between the talus and navicular bones in the joint plane were determined. The relative translations and rotations between the bones in the joint frame are depicted in Figures 43 to 48 . The results were compared for statistical difference using SAS as described earlier. The results of the statistical tests are described below along with the graphs.

Figures 43 to 45 show the relative translations between the talus and navicular bone along each axis of the joint frame. In each of the three graphs, the vertical axis represents relative translation in millimeters. Statistical tests were performed on translation results in the joint frame and significant differences were found. All significant differences were in translations along $\mathrm{X}^{\prime}$ axis of joint frame which represents pure separation between the articulating surfaces. There was a significant difference reported between the two groups during internal rotation tests pre-operatively i.e., in harvested condition. The $\mathrm{P}$ value was 0.03 for internal rotation sequential test and 0.04 for internal rotation simultaneous test. In the harvested condition the plate screw group showed more motion as compared to the screws group. On comparing the two screws group and plate screw group post-surgically, the plate screw group showed less motion along $X^{\prime}$ axis as compared to two screws group during internal rotation sequential test with a $P$ value of 0.07 . No comparison was statistically significant at level of 0.05 . 


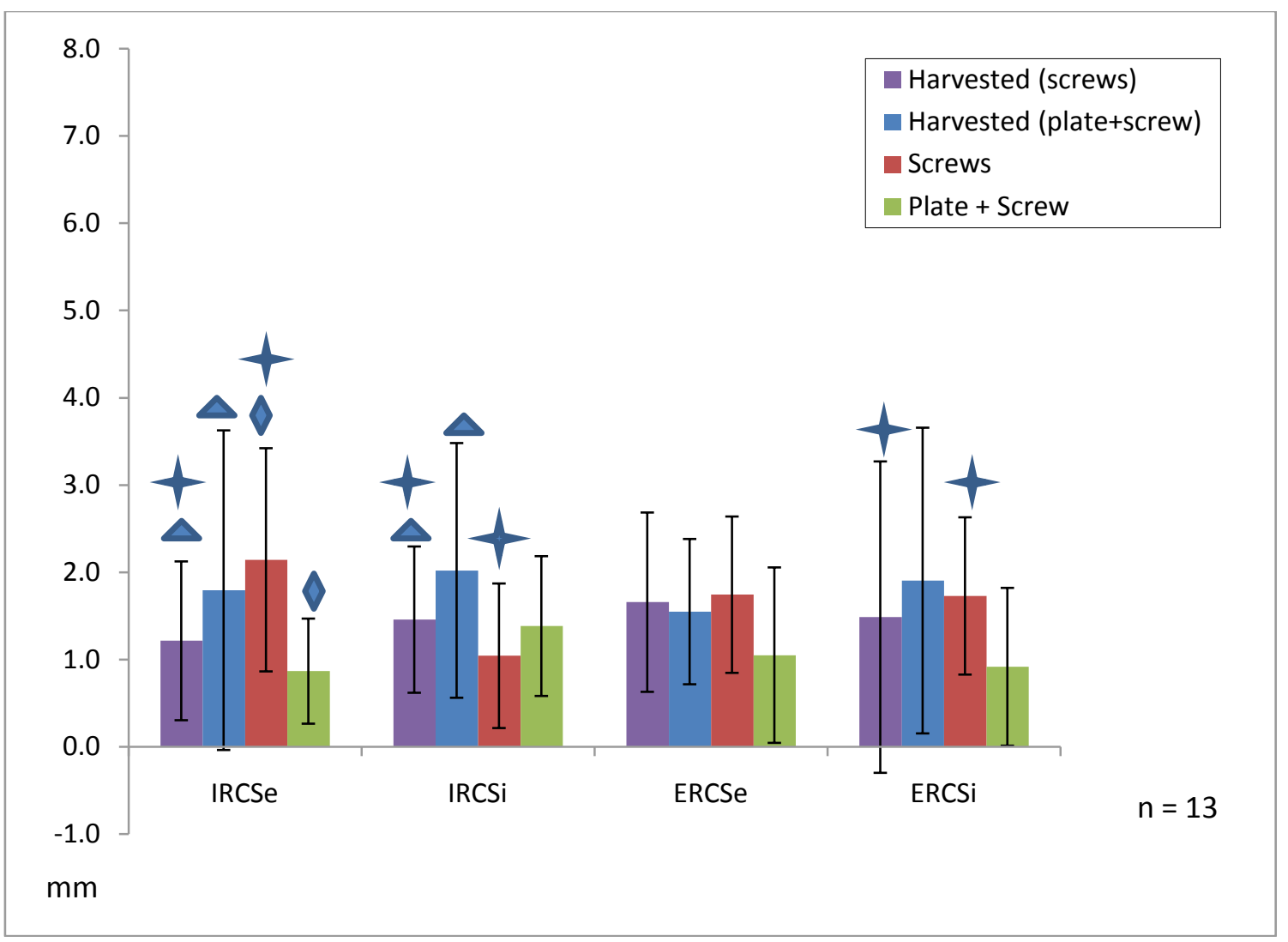

Figure 43: $\quad$ Relative translation along $X^{\prime}$ axis (joint frame)

Note: $\Delta=$ Significant difference between pre-operative groups.

- Significant difference between post-operative groups.

$+=$ Significant difference between pre-operative and postoperative state of plate screw group. 


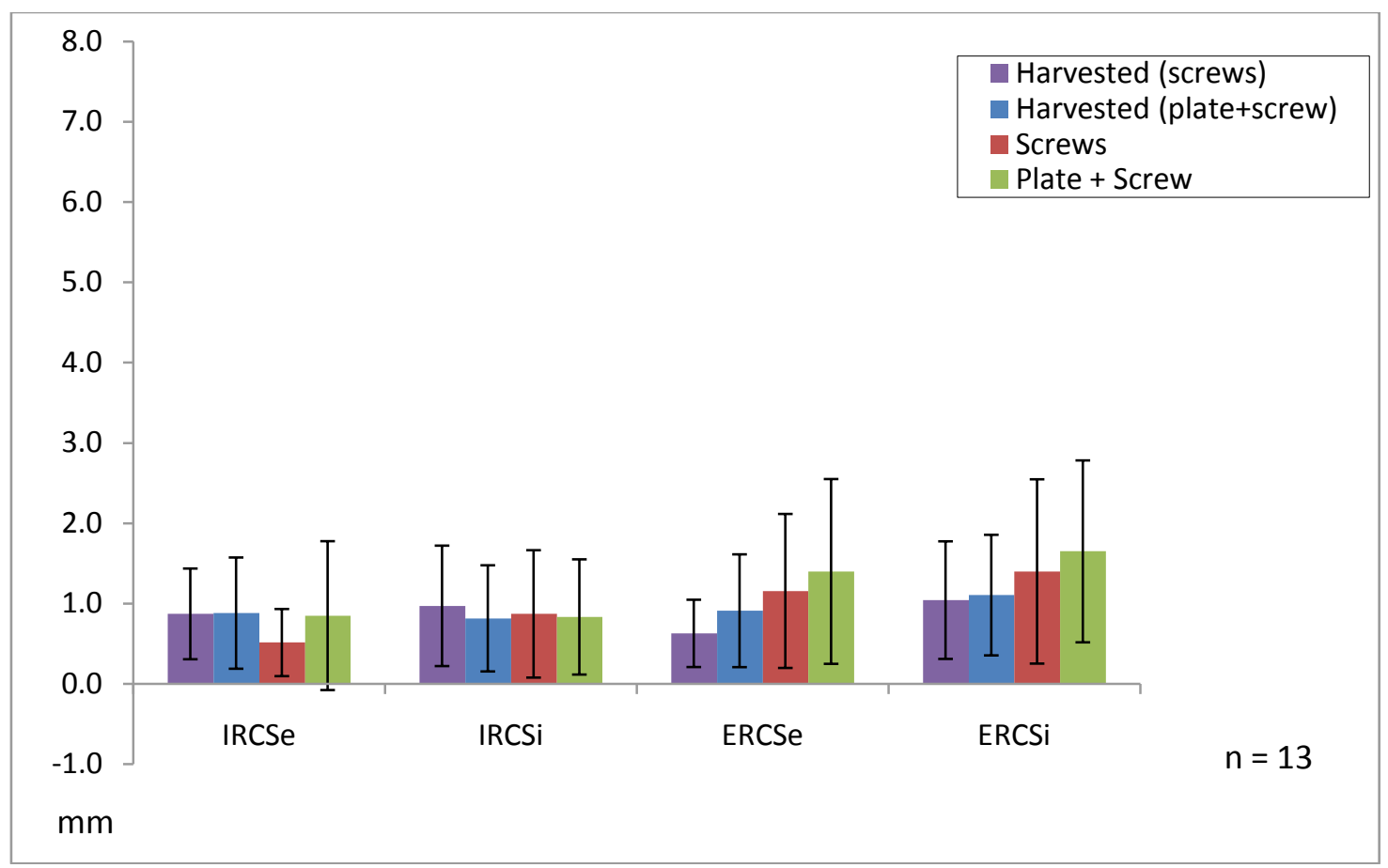

Figure 44: $\quad$ Relative translation along $Y^{\prime}$ axis (joint frame) 


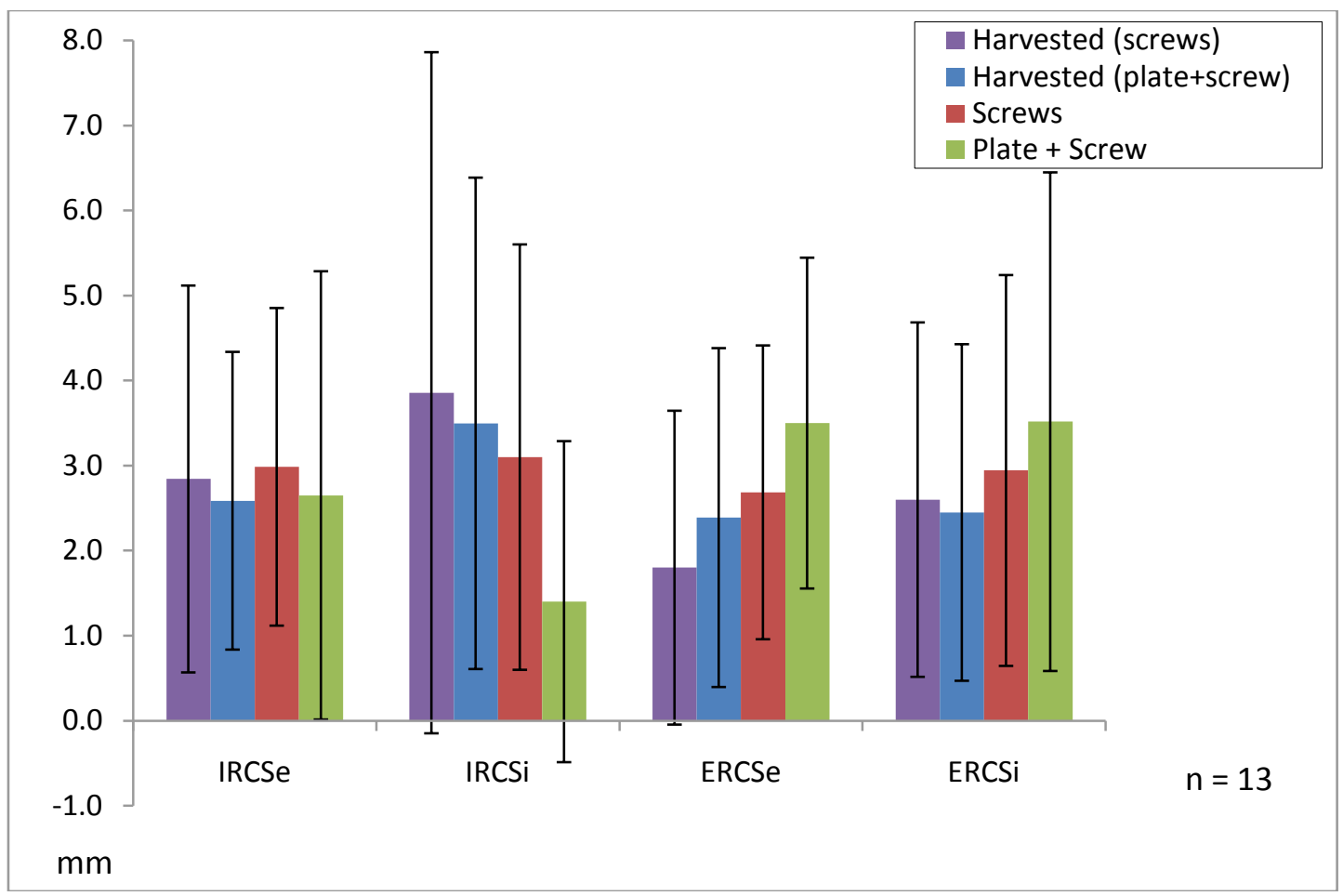

Figure 45: $\quad$ Relative translation along $Z^{\prime}$ axis (joint frame) 


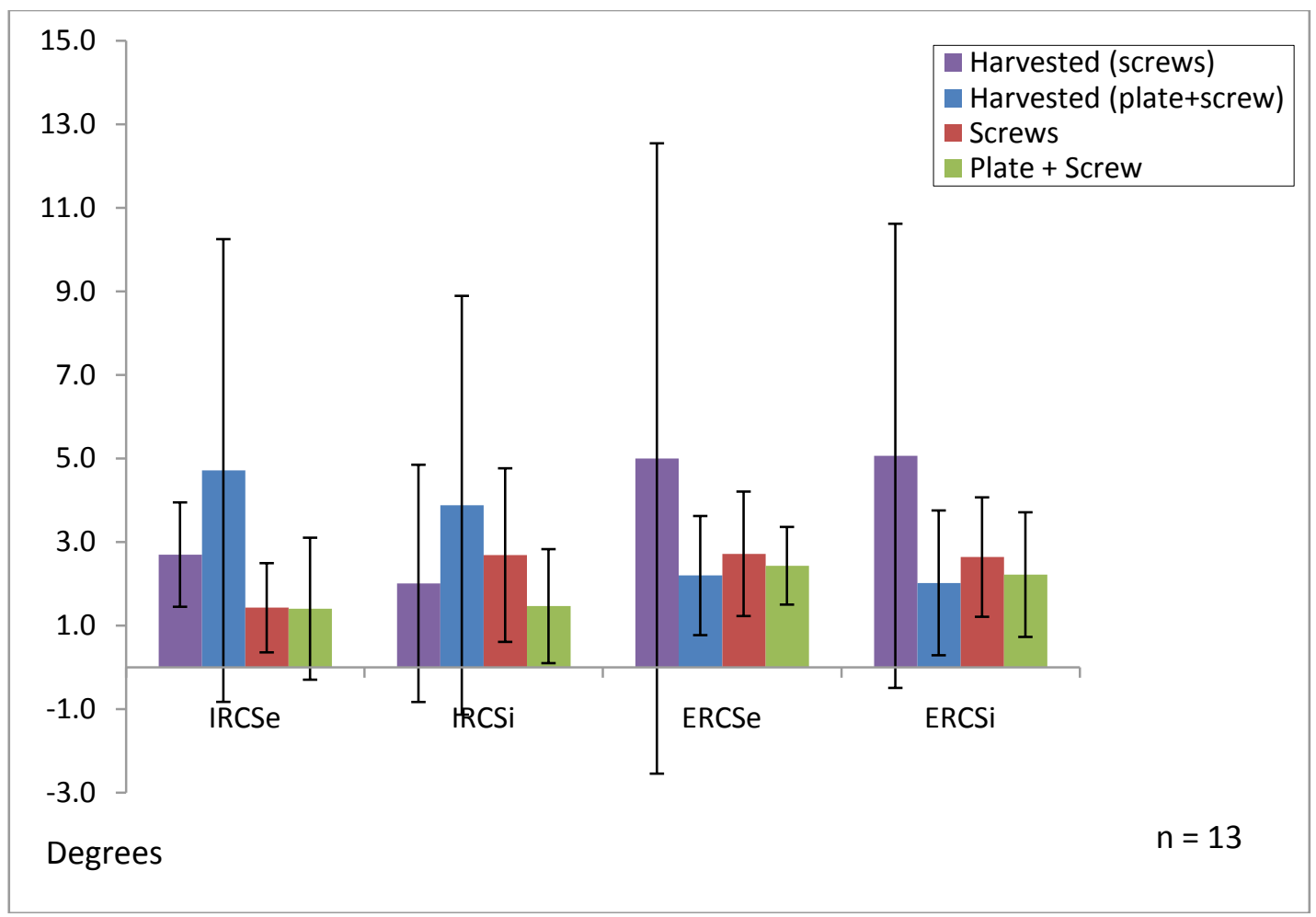

Figure 46: Relative rotation about $X^{\prime}$ axis (joint frame) 


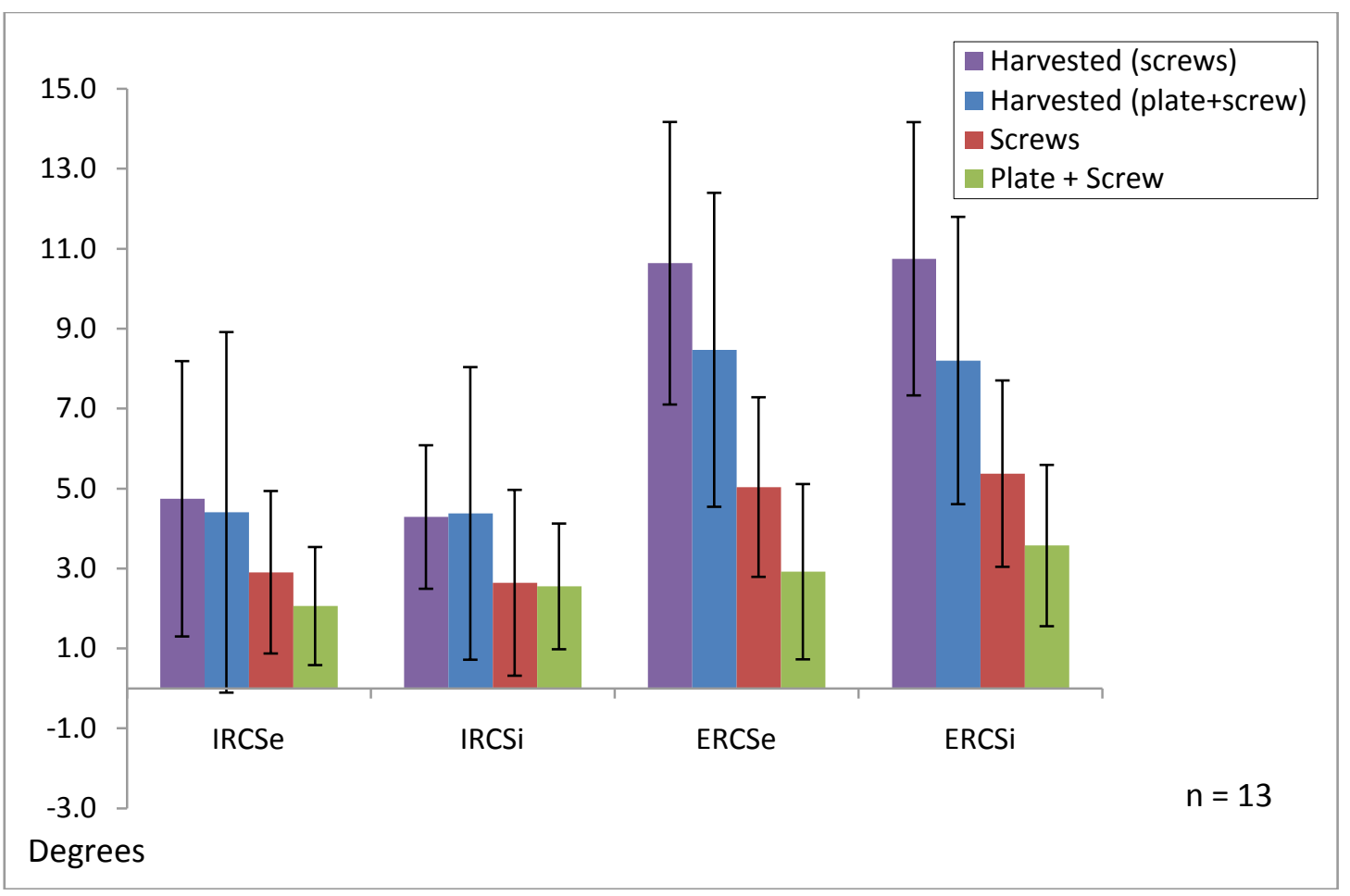

Figure 47: $\quad$ Relative rotation about $Y^{\prime}$ axis (joint frame) 


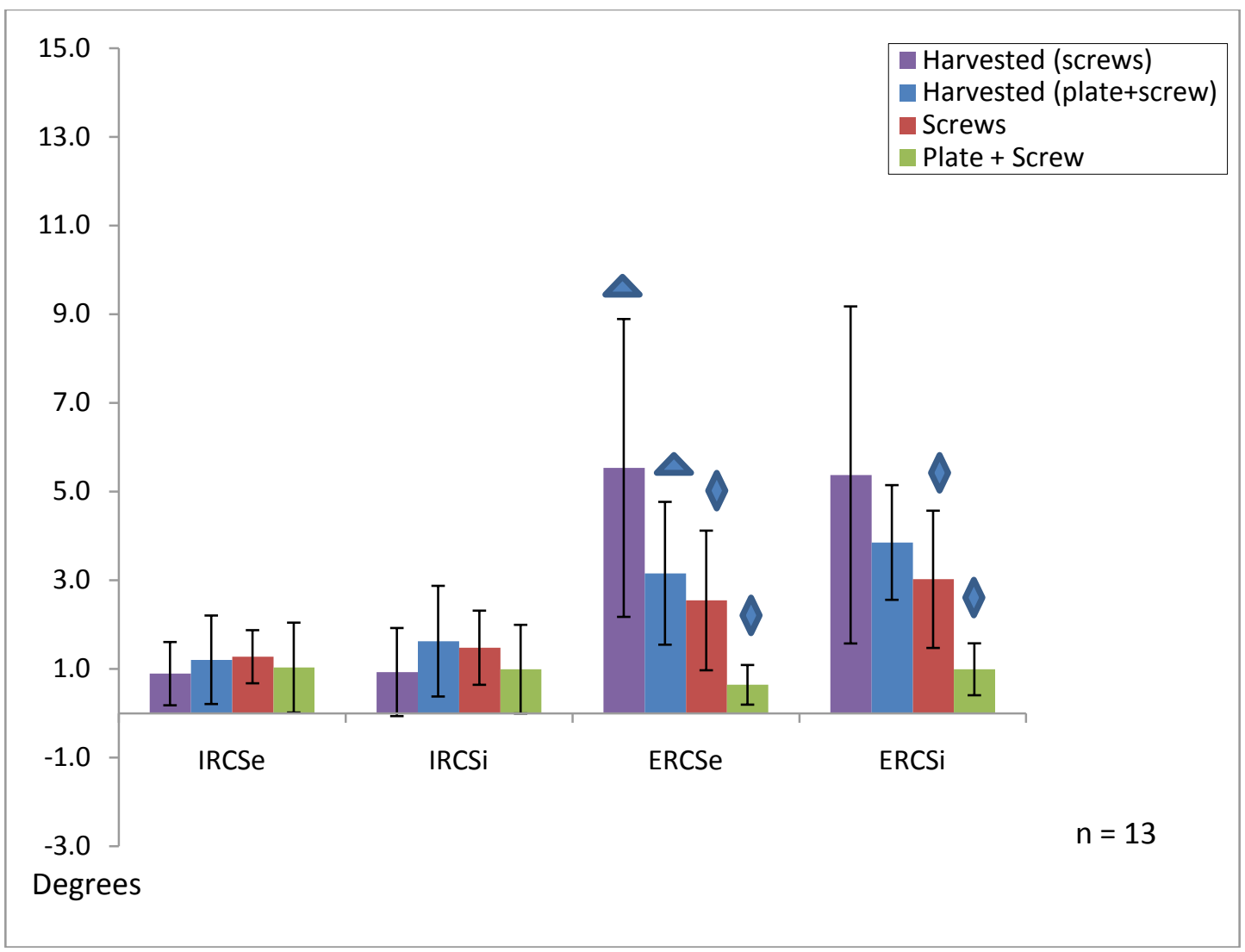

Figure 48: $\quad$ Relative rotation about $Z^{\prime}$ axis (joint frame)

Note: $\Delta=$ Significant difference between pre-operative groups.

- $=$ Significant difference between post-operative groups. 
However, on comparing the harvested and instrumented conditions, the plate screw group showed significantly less motion as compared to harvested condition. The $\mathrm{P}$ values were 0.007 for internal rotation sequential test, 0.02 for internal rotation simultaneous test and 0.05 for external rotation simultaneous test. The two screws group didn't show any statistically significant difference between harvested and instrumented condition. The plate plus one screw reduced the translation along $\mathrm{X}^{\prime}$ axis from a preoperative translation of $1.8 \mathrm{~mm}$ (internal rotation sequential test), $2.0 \mathrm{~mm}$ (internal rotation simultaneous test), and $1.9 \mathrm{~mm}$ (external rotation simultaneous test) to $0.9 \mathrm{~mm}$, $1.4 \mathrm{~mm}$, and $0.9 \mathrm{~mm}$ respectively. There was no statistically significant difference in the translations along $\mathrm{Y}^{\prime}$ and $\mathrm{Z}^{\prime}$ axes of joint frame.

Figures 46 to 48 show the relative rotations between the talus and navicular bone about each axis of the joint frame. In each of the three graphs, the vertical axis represents relative rotation in degrees. The rotations about $\mathrm{X}^{\prime}, \mathrm{Y}^{\prime}$ and $\mathrm{Z}^{\prime}$ axes of joint frame were compared for statically significant difference. Pre-operatively the plate screw group and two screws group showed statistically significant difference in rotations about $Z$ ' axis during external rotation sequential test. The $\mathrm{P}$ value was 0.04 . The plate screw group showed less rotation as compared to the two screws group.

Post-operatively there was a highly significant difference in rotations about $Z^{\prime}$ axis between two screws group and plate screw group during external rotation sequential test and during external rotation simultaneous test. The $\mathrm{P}$ values were 0.002 and 0.001 . There was also a difference between the rotations about $\mathrm{Y}^{\prime}$ axis during external rotation sequential test. The $\mathrm{P}$ value was 0.07 . In all these cases, the plate screw group showed less rotation as compared to two screws group. The rotation of the plate screw group was 0.6 degrees during external rotation sequential test as compared to 2.5 degrees in the two screws group and it was 1 degree during external rotation simultaneous test in the plate screw group as compared to 3 degrees in the two screws group. This rotation represents the flexion-extension motion at the joint. Thus the plate plus one screw type of instrumentation may be able to restrict the flexion extension motion more effectively than two screws type instrumentation. There was no statistically significant difference between any of the other groups. 


\section{CHAPTER 6: DISCUSSION AND CONCLUSIONS}

\section{DISCUSSION}

Selective arthrodesis is growing in popularity for treatment of hind foot diseases. The main problem encountered in talonavicular fusion, is the high rate of non union. The general objective of this study was to find a potential cause of failure of talonavicular joint fusion. The specific objective was to compare the two types of fixation techniques and instrumentations, namely, two screws versus a locked compression plate plus one screw. Specifically, the rotational and translational motion occurring at the joint before and after fixation using these instrumentations was studied.

We observed that in the native state, the maximum relative translation was in $Z^{\prime}$ axis direction, i.e., the medial-lateral motion and the maximum rotation was in adductionabduction direction. This implies that the joint is most unstable on the medial aspect, which is in keeping with the anatomy since this joint is subcutaneous medially and has least support on the medial side. Thus any possible fixation technique should take these factors into consideration.

The motion of the bones was recorded using targets attached to the talus and navicular bones. The motion was then transformed to a point $J$ in the talonavicular joint space and all motion was described as occurring at this point. In most published papers, the gross motion of the bone is described in a global reference frame aligned with the sagittal, coronal and transverse planes of the body. $[1,2,18]$ The advantage of transforming the motion data to the joint space is that it provides a better understanding of the local motion at the site of healing which would have a direct bearing on the success of the procedure. It should be noted that when the motion occurring at the joint plane is analyzed, it is seen that any motion is total of its components along three orthogonal axes. The choice of these axes is important. If the axes are chosen to align with the global planes such as sagittal plane, coronal plane or transverse plane, then if any motion occurs along the joint plane, only a component of it can be seen in line with the global axes and the exact motion occurring at the joint cannot be correctly estimated.

In our study, the motion was transformed into a joint frame which was aligned with the long axis of the talus and the joint line. Thus the data reported in this study provides a more accurate estimate of the motion occurring at the joint. This finding was substantiated by the results of the study. In the camera frame there was no significant difference in the translation data along any axis. However when the same data was transformed into the joint frame, there was significant difference along $\mathrm{X}^{\prime}$ axis in the plate screw group as compared to harvested in three out of the four tests. The two screws group did not show any statistically significant difference in translation. Thus by transforming the translations into the joint frame, the benefit of the fixation using plate plus one screw type of instrumentation instead of two screws instrumentation could be determined. Further, this relative translation along $\mathrm{X}^{\prime}$ axis represented the true separation along the long axis of the talus and thus the actual physical gap between the two bones. 
Since fusion of joint after removal of cartilage and fixation using an internal fixation device could occur by a similar mechanism to fracture healing, the factors affecting fracture healing could be extrapolated to joint fusion. It is known that some amount of motion between two sides of a fracture is required for formation of callus and ultimate healing of fracture.[30] Thus, it can be said that some amount of motion between the two bones should be present. However it is also known that rate of healing is inversely proportional to the physical gap between two bones, i.e., smaller the gap, faster the fusion. [31, 32] Thus in case of joint fusion, the increase in the physical gap between the bones may delay the healing process while the micro-motion in other directions which occurs without increasing the physical gap between the two bones may be beneficial to fusion. The methodology developed in this study has the ability to estimate motion along the axes aligned with the joint plane and along the long axis of the bone and can predict motion along the long axis of the bone as well as the other axes. The results of this study could therefore indicate the possibility of successful fusion and thus have direct clinical relevance.

It was observed that, the rotations about the global, i.e., camera frame showed statistically significant difference pre-operatively about the $\mathrm{X}$ axis during external rotation tests. Post-surgically the rotations were significantly different about $\mathrm{Z}$ axis during the external rotation tests with $P$ values of 0.0002 and less than 0.0001 . However when the data was converted into the joint frame, the rotations were significantly different about $Z^{\prime}$ axis during the external rotation tests with $P$ values of 0.002 and 0.001 . The plate screw group showed less motion as compared to two screws group. Thus by transforming into the joint frame, overestimation of the benefit of using plate instead of screws was avoided. Thus the transformation of data into joint frame brings out the actual translational and rotational motion occurring at the joint and provides an insight into the local joint mechanics without overestimating the benefit of any fixation method. This procedure would lead to better understanding of local joint mechanics and be helpful in designing and developing implants.

The data obtained in this study in the joint frame suggested that the mean relative separation along $\mathrm{X}^{\prime}$ axis is significant less in the plate screw group as compared to harvested in case of internal rotation sequential, internal rotation simultaneous and external rotation simultaneous tests. Thus, the plate plus one screw type of instrumentation is effective in reducing the relative separation between the bones and may prove to be useful for accelerating the fusion process. The plate screw group also showed highly significant reduction in rotation about $Z$ axis i.e., flexion and extension motion as compared to two screws group. Thus the plate plus one screw technique may prove beneficial in partially non-complaint patients who subject the post-operative foot to varying amount of load bearing.

The data obtained regarding the remaining axes ( $\mathrm{Y}^{\prime}$ and $\mathrm{Z}^{\prime}$ for translation and $\mathrm{X}^{\prime}$ and $Y^{\prime}$ for rotation) showed no statistically significant difference between the plate screw group and the two screws group. Thus the relative translation and rotation between the two bones would be similar along these axes irrespective of the type of implant used. Pre-operatively, the translation motion along the $\mathrm{Y}^{\prime}$ axis (vertical separation) was in the 
range of 0.8 to $1.9 \mathrm{~mm}$ and along $\mathrm{Z}^{\prime}$ axis medial-lateral motion) was between 1.8 to 3.9 $\mathrm{mm}$. Post-operatively, the translation along $\mathrm{Y}^{\prime}$ axis was between 0.5 to $1.7 \mathrm{~mm}$ and along $\mathrm{Z}^{\prime}$ axis was between 1.4 to $3.5 \mathrm{~mm}$. Thus there was a marginal reduction in the translation motion post-operatively. However, the translation was not entirely eliminated. Similarly, the pre-operative rotations about $\mathrm{X}^{\prime}$ axis (inversion-eversion) was between 2 to 5.1 degrees pre-operatively and 1.4 and 2.7 degrees post-operatively. The mean rotations about $\mathrm{Y}^{\prime}$ axis (adduction-abduction) were between 4.3 to 10.7 degrees pre-operatively and 2.1 and 5.0 degrees post-operatively. Once again there was a reduction in the rotational motion but rotation was not entirely eliminated. However, as previously stated, if the fixation is too tight, then it may hinder formation of callus and thus hinder healing process. Thus the motion seen in thus study may not be counterproductive to healing.

Also, it must be noted that the motion seen in this study is as a result of the loads applied to the specimens, which were a lot more than physiological loads in such cases. Typically, a patient who has undergone talonavicular joint fixation is put in a cast and instructed against weight-bearing on that foot. Thus the patient would typically not put his/her entire body weight on the foot. Additionally, the cast would prevent sudden and excessive rotation of the foot. The conditions applied in this study which included an Achilles tendon load of $350 \mathrm{~N}$, a compressive load of $850 \mathrm{~N}$ and a rotation of 15 degrees or $10 \mathrm{Nm}$ of torque would be far more than those actually experienced by patient postoperatively. Thus, this study has tested the ability of the implants to withstand unprotected weight-bearing and what could be called as the worst case scenario. Considering this, the actual motion between the bones in clinical setting would be less as compared to that in this study and the motion would therefore not hinder fusion process. Though rigid fixation has been known to benefit fusion, the amount of motion that prevents fusion has not been established in any study.[18] It was therefore assumed that the motion seen in this study doesn't preclude fusion and the instrumentation which allows lesser motion would be more likely to produce fusion of the talonavicular joint.

The study simulated loading scenarios which involved constant contact of the sole of the foot to the base plate. Thus, these scenarios were representative of static weight bearing or the mid-stance phase of the gait cycle. The effect of loading during other phases of the gait cycle was not studied. However as stated above, the patient is typically in a cast after the procedure and hence is unable to perform dorsiflexion or plantar flexion. Thus in this respect, the loading conditions in this study are representative of the actual clinical conditions.

The loading scenarios involved sequential loading scenarios (internal or external rotation followed by compression). It was observed that the internal or external rotation put all the bones of the foot in a specific orientation and locked them in that position before the compressive load was applied. In physiological setting, the ligaments and tendons stabilize the arches of the foot and allow the foot to withstand large loads. Thus the sequential loading scenarios were representative of the natural loading occurring in the foot. The simultaneous loading scenarios involved rotation and compressive forces 
simultaneously and can be said to be representative of sudden unexpected or traumatic motion as occurs during twisting of ankle, or other traumatic event.

During this study, the muscles and tendons except the Achilles tendon were not actively controlled. In physiological setting, the muscles and tendons are responsible for the motion at the joint. In this study, the motion of the joint was produced artificially using the multi-axis robot. However the end point of motion and loading was replicated and thus the results at the end point/ loading limit would be comparable irrespective of the method used to reach the end limit. Thus the result of loading achieved by using the robot would be comparable to the natural loading by muscles and tendons.

During this study, there was no active dorsiflexion or plantarflexion motion of the foot. The supination motion of the foot is more pronounced during dorsiflexion while pronation more pronounced during plantar flexion. The absence of dorsiflexion and plantar flexion in this study may be partially responsible for the smaller amounts of rotation seen at the talonavicular joint as compared to published literature.[1] However, most of these published studies study the joint range of motion under non-weight bearing conditions. In our study, the motion was replicated under load bearing conditions which mimics the real life loading scenarios and thus smaller range of motion may not be unexpected.

A limitation of this study was the small sample size. This could be improved by increasing the sample size. However, the cost of procuring, processing and testing of these human cadaveric specimens is considerable and increasing the specimens would require additional expenditure. At the end of such a study with increased sample size, similar results may be obtained as the current results and additional expenditure may not be justified. Hence, it was decided that a sample size of 13 as used in this study would be considered adequate.

In this study, a procedure was developed to identify a point $\mathrm{J}$ inside the talonavicular joint space. The procedure was used consistently for all specimens. However due to errors in radiographic views and processing, it must be mentioned that there could have been some error in defining the exact position of point $J$. Point $J$ was to be placed at the summit of the convex surface of the head of the talus but the position may have an absolute error of up to $5 \mathrm{~mm}$. However, once the position of point $\mathrm{J}$ is defined, all motion is described relative between the two bones and the description of the motion is itself independent of the location of point $J$. Thus any error in identifying point $\mathrm{J}$ does not affect the final results.

Thus, the results reported would be affected by the resolution of the camera, the accuracy of various measurements and data processing errors due to use of trigonometric functions i.e., sines and cosines. The resultant accuracy of the results would be in the order of $10^{-1} \mathrm{~mm}$. In clinical practice, the motion or separation which can be discerned on a radiograph is usually not less than $0.1 \mathrm{~mm}$. The resolution of the results of this study is at least at par with other published studies[3] and the errors would not limit the application of these results in clinical practice. 
The study was done on specimens, which were exposed to air. Also the specimen were frozen between the tests However, the results obtained would not be affected by this, since it is known that the mechanical properties of the bone are unaffected by exposure to air or freezing as long as the specimen is kept moist using a physiological solution such as saline used in this study.[33]

The age range for the specimens used in this study was from 52 to 75 years. The typical age group affected by arthritic conditions requiring this type of surgery is $50+$ age group. Since out of each pair of feet, one foot was allocated to each of the two groups, age bias between the two groups should not be an issue. There was a female preponderance in the pool of specimens. Five out of the seven pairs of feet were from female cadavers. Rheumatoid arthritis, which is the common indicator for talonavicular arthrodesis, is common in females.[7, 16, 34] Since the pairs were split up equally, there was no sex bias between the two groups. Thus the specimens were representative of the affected population with respect to age and sex. The demographic data of the specimens is summarized in Appendix $\mathrm{H}$.

There was an inadvertent mismatch in the left feet to right feet ratio in each group and there was a preponderance of left feet in plate screw group and right feet in the two screws group. However since the results of the harvested testing were analyzed and the post-surgical motion was compared with the pre-surgical motion, any bias introduced into the study due to this mismatch was minimized. Comparison of post-surgical with presurgical data also accounted for any bias due to differences between the two groups presurgically.

During the process of specimen selection, only healthy specimens were chosen. The specimens having deformity would have required additional surgery to correct the deformity. It was the objective of the study to keep the surgical procedure constant and hence only healthy specimens in which the standard surgery could be performed were selected for this study. The surgical procedure was performed by a single surgeon, which eliminated interpersonal variability in the procedure or fixation technique.

During the surgical procedure it was observed that some specimens showed poor bone quality and it was difficult to get sufficient purchase for the screws requiring multiple attempts and/or changing orientation of the screws. Also, the feet specimens were of varying sizes with some being large as 13 inches in length, while others were as short as 8 inches. However the size of the implants used was standard. These could have been some of the contributory factors to the relative laxity of the joints post-operatively. Thus, there may be a case for customizing the size of implants.

Due to the difference in the size of the feet, it was possible that the internalexternal rotations produced during testing would occur about different regions of the foot. This was avoided by the use of the XY table which allowed the foot to slide in the X-Y plane and thus internal-external rotation occurred about the natural center of rotation for each foot irrespective of the size and shape. 
The processing of the specimens during this study was similar to the procedure used in actual clinical cases. For example, a patient requiring talonavicular joint surgery would be examined and investigated prior to surgery to determine the pre-operative condition. This step is similar to the harvested condition testing used in this study. The immediate post-operative results in the patient would be similar to the results of the postinstrumentation testing in this study. Thus the results of this study could be correlated with actual clinical cases. This study was thus able to compare two fixation techniques used for talonavicular joint fusion and provide clinically relevant results.

\section{CONCLUSIONS}

1. A cadaveric foot model was developed to study the biomechanics of the talonavicular joint.

2. Using this model, it was possible to describe the motion occurring at the talonavicular joint as a result of various loading conditions.

3. Two types of fixation techniques used to produce talonavicular joint fusion were compared using this model to determine the efficacy of these two techniques in reducing the motion at the joint. It was determined that the locked compression plate plus one retrograde screw instrumentation was more effective in reducing separation along the long axis of talus compared to harvested state and this instrumentation resists dorsiflexion-plantar flexion type rotation compared to the two screws type instrumentaion. Based on the results, it may be predicted that, the plate plus one screw may afford a little better fixation in partially noncompliant patients who subject the post-operative foot to partial weight bearing.

4. The foot model provides a relatively fast and convenient method to investigate the effect of various foot conditions or surgical procedures or instrumentation.

5. The methodology of transforming motion information into joint planes provides a better understanding of the motion at joint and provides true estimates of the joint motion and thus provides clinically relevant data. 


\section{CHAPTER 7: RECOMMENDATIONS FOR FUTURE WORK}

This study was done using a multi-axis programmable robot. In this study, only vertical compression and internal and external rotation was produced. The robot has four degrees of freedom and is capable of producing flexion-extension motion about any point as well as axial twist. The robot has a feature called tool tip transformation, which allows precise control of loading and motion using any point in space as a reference. Thus using the complete potential of the robot, the effect of loading as occurs during various stages of gait cycle could be studied by programming the multi-axis programmable robot to replicate the gait cycle. The use of a pressure sensor plate below the foot can be used to validate the gait model by comparing the pressure patterns of cadaveric model to in vivo gait studies. Also, the effect of active dorsiflexion and plantar-flexion motion can be studied by producing the complete range of motion similar to normal human gait.

The model could be expanded to analyze local strain patterns in the bones. This would involve measurement local forces in the joint.

Studies could also be designed to investigate the effect of triple joint fusion versus isolated talonavicular fusion or double fusion. In addition, the effect of changing orientation of the plate and screw could be studied to determine the optimal surgical placement of the instrumentation.

The contribution of the surgical debridement to the joint instability could also be the focus of a separate study. This would involve testing of specimen in the intact state followed by insertion of instrumentation without debridement or removal of cartilage. The results obtained by testing specimen instrumented in this manner could be compared with the results obtained in our present study to determine the contribution of surgical debridement towards joint instability.

The effect of cyclical loading on the motion of bones before and after fixation could also be studied. This type of study would require baseline cyclical testing data of the intact condition as well as cyclical data of instrumented condition. There is a possibility of degradation of tissue quality during cyclical testing of intact state rendering the specimen unusable for instrumentation. Thus it may become necessary to perform instrumentation of specimens which have not been subjected to cyclical testing and the instrumented and harvested groups may thus contain different specimens.

The foot model developed in this study could be used for studying other joints of the foot, such as the subtalar or the calcaneocuboid joint or any other joint of the body using a similar procedure. By identifying an appropriate point inside the joint similar to point $\mathrm{J}$ in this study, the relative motion between any two bones can be determined.

The methodology developed in this study, which allows for reduction motion data of two targets attached to two bones to a point inside the joint space between the bones, 
can be extended to track multiple joints. It is also possible to describe the motion with respect to a single global reference frame or along individual joint frames which allows comparison between the published global motion data and actual motion in the joint coordinate frame. This arrangement would also provide data regarding real time tracking of bones, which could be used to create computer based models of the foot. 


\section{LIST OF REFERENCES}

1. Savory K, Wülker N, Stukenborg C, Alfke D: Biomechanics of the hindfoot joints in response to degenerative hindfoot arthrodeses. Clin Biomech (Bristol, Avon) 1998; $13: 62$

2. Wülker N, Stukenborg C, Savory K, Alfke D: Hindfoot motion after isolated and combined arthrodeses: measurements in anatomic specimens. Foot Ankle Int 2000;21:921

3. Astion D, Deland J, Otis J, Kenneally S: Motion of the hindfoot after simulated arthrodesis. J Bone Joint Surg Am 1997;79:241

4. Harper M, Tisdel C: Talonavicular arthrodesis for the painful adult acquired flatfoot. Foot Ankle Int 1996;17:658

5. Bibbo C, Anderson R, Davis W: Complications of midfoot and hindfoot arthrodesis. Clin Orthop Relat Res 2001;391:45

6. Chiodo C, Martin T, Wilson M: A technique for isolated arthrodesis for inflammatory arthritis of the talonavicular joint. Foot Ankle Int 2000;21:307

7. Elbar J, Thomas W, Weinfeld M, Potter T: Talonavicular arthrodesis for rheumatoid arthritis of the hindfoot. Orthop Clin North Am 1976;7:821

8. Lombardi C, Dennis L, Connolly F, Silhanek A: Talonavicular joint arthrodesis and Evans calcaneal osteotomy for treatment of posterior tibial tendon dysfunction. J Foot Ankle Surg 1999;38:116

9. Ljung P, Kaij J, Knutson K et al.: Talonavicular arthrodesis in the rheumatoid foot. Foot Ankle 1992;13:313

10. Chen $\mathrm{C}$, Huang $\mathrm{P}$, Chen $\mathrm{T}$ et al.: Isolated talonavicular arthrodesis for talonavicular arthritis. Foot Ankle Int 2001;22:633

11. Fogel G, Katoh Y, Rand J, Chao E: Talonavicular arthrodesis for isolated arthrosis: 9.5-year results and gait analysis. Foot Ankle 1982;3:105

12. Angus $\mathrm{P}$, Cowell H: Triple arthrodesis. A critical long-term review. J Bone Joint Surg Br 1986;68:260

13. Bennett G, Graham C, Mauldin D: Triple arthrodesis in adults. Foot Ankle $1991 ; 12: 138$

14. Figgie M, O'Malley M, Ranawat $\mathrm{C}$ et al.: Triple arthrodesis in rheumatoid arthritis. Clin Orthop Relat Res 1993;292:250 
15. Graves S, Mann R, Graves K: Triple arthrodesis in older adults. Results after long-term follow-up. J Bone Joint Surg Am 1993;75:355

16. Donatto K: Arthritis and arthrodesis of the hindfoot. Clin Orthop Relat Res 1998;349:81

17. Jarrell Sr, Owen J, Wayne J, Adelaar R: Biomechanical comparison of screw versus plate/screw construct for talonavicular fusion. Foot Ankle Int 2009;30:150

18. Rosenfeld J, Parks B, Schon L: Biomechanical investigation of optimal fixation of isolated talonavicular joint fusion. Am J Orthop (Belle Mead NJ) 2005;34:445

19. Suckel A, Muller O, Herberts T et al.: Talonavicular arthrodesis or triple arthrodesis: peak pressure in the adjacent joints measured in 8 cadaver specimens. Acta Orthop 2007;78:592

20. Sommer C, Gautier E, Müller $M$ et al.: First clinical results of the Locking Compression Plate (LCP). Injury 2003;34 Suppl 2:B43

21. Schütz M, Südkamp N: Revolution in plate osteosynthesis: new internal fixator systems. J Orthop Sci 2003;8:252

22. Smith W, Ziran B, Anglen J, Stahel P: Locking plates: tips and tricks. J Bone Joint Surg Am 2007;89:2298

23. Frigg R: Locking Compression Plate (LCP). An osteosynthesis plate based on the Dynamic Compression Plate and the Point Contact Fixator (PC-Fix). Injury 2001;32 Suppl 2:S-B63

24. Fröberg A, Komi $\mathrm{P}$, Ishikawa $\mathrm{M}$ et al.: Force in the achilles tendon during walking with ankle foot orthosis. Am J Sports Med 2009;37:1200

25. Nordin M, Frankel VH: Basic biomechanics of the musculoskeletal system. (ed. 3rd). Philadelphia: Lippincott Williams \& Wilkins, 2001

26. Roth R: Talonavicular joint osteoarthritis (osteoarthrosis). J Am Podiatry Assoc $1982 ; 72: 237$

27. Weinstein SL, Buckwalter JA, Turek SL: Turek's Orthopaedics : principles and their application. (ed. 5th). Philadelphia: Lippincott, 1994

28. Mann RA, Coughlin MJ: Surgery of the foot and ankle. (ed. 6th). St. Louis: Mosby, 1993

29. Harper M: Talonavicular arthrodesis for the acquired flatfoot in the adult. Clin Orthop Relat Res 1999;365:65 
30. Jagodzinski M, Krettek C: Effect of mechanical stability on fracture healing--an update. Injury 2007;38 Suppl 1:S3

31. Claes L, Heigele C, Neidlinger-Wilke $\mathrm{C}$ et al.: Effects of mechanical factors on the fracture healing process. Clin Orthop Relat Res 1998;355S:S132

32. Lacroix D, Prendergast P: A mechano-regulation model for tissue differentiation during fracture healing: analysis of gap size and loading. J Biomech 2002;35:1163

33. An YH, Draughn RA: Mechanical testing of bone and the bone-implant interface. Boca Raton: CRC Press, 2000

34. Kitaoka H: Rheumatoid hindfoot. Orthop Clin North Am 1989;20:593 


\section{APPENDIX A: DESIGNS}

\section{ACHILLES TENDON LOADING SYSTEM}

\section{Objective}

To deliver a constant load to the Achilles tendon.

\section{Device Description and Action}

The Achilles tendon is the largest tendon in the body and capable of withstanding about 2.1 times body weight.[24] It is located on the posterior side of leg. It originates from the gastrocnemius and soleus in the lower half of the leg and curves around itself before insertion into the heel bone. The line of action of force is parallel to the tibia. It was important to conserve this line of action to replicate physiological loading scenarios.

As seen in Figure 49, a cable was passed over two pulleys, which were supported by aluminum framework. This frame device was mounted on the robot platform such that one pulley was directly above the Achilles tendon while other extended away from the platform. A cable passed over these two pulleys was attached to the Achilles tendon using a cable puller and a weight plate was suspended from the other end of the cable to hang free from the platform. Thus any weight added to the weight plate produced a vertical tension force on the Achilles tendon and the physiological line of action of Achilles tendon was preserved.

\section{XY TABLE}

\section{Objective}

To allow free motion of foot specimen in XY plane.

\section{Device Description and Action}

Two low friction slides were procured (Parker product numbers: SE100A-200 and SE100A-350). One of the slides had $150 \mathrm{~mm}$ travel and was $100 \mathrm{~mm}$ in breadth and capable of withstanding a torque of up to $44 \mathrm{Nm}$. The other slide had $300 \mathrm{~mm}$ travel and was $100 \mathrm{~mm}$ in breadth and capable of withstanding a torque of up to $82 \mathrm{Nm}$. These slides were assembled using mating plates and placed between the foot platform (which was a rectangular plate of aluminum) and the turn table. The completely assembled XY table on the turn table is shown in Figure 50. 


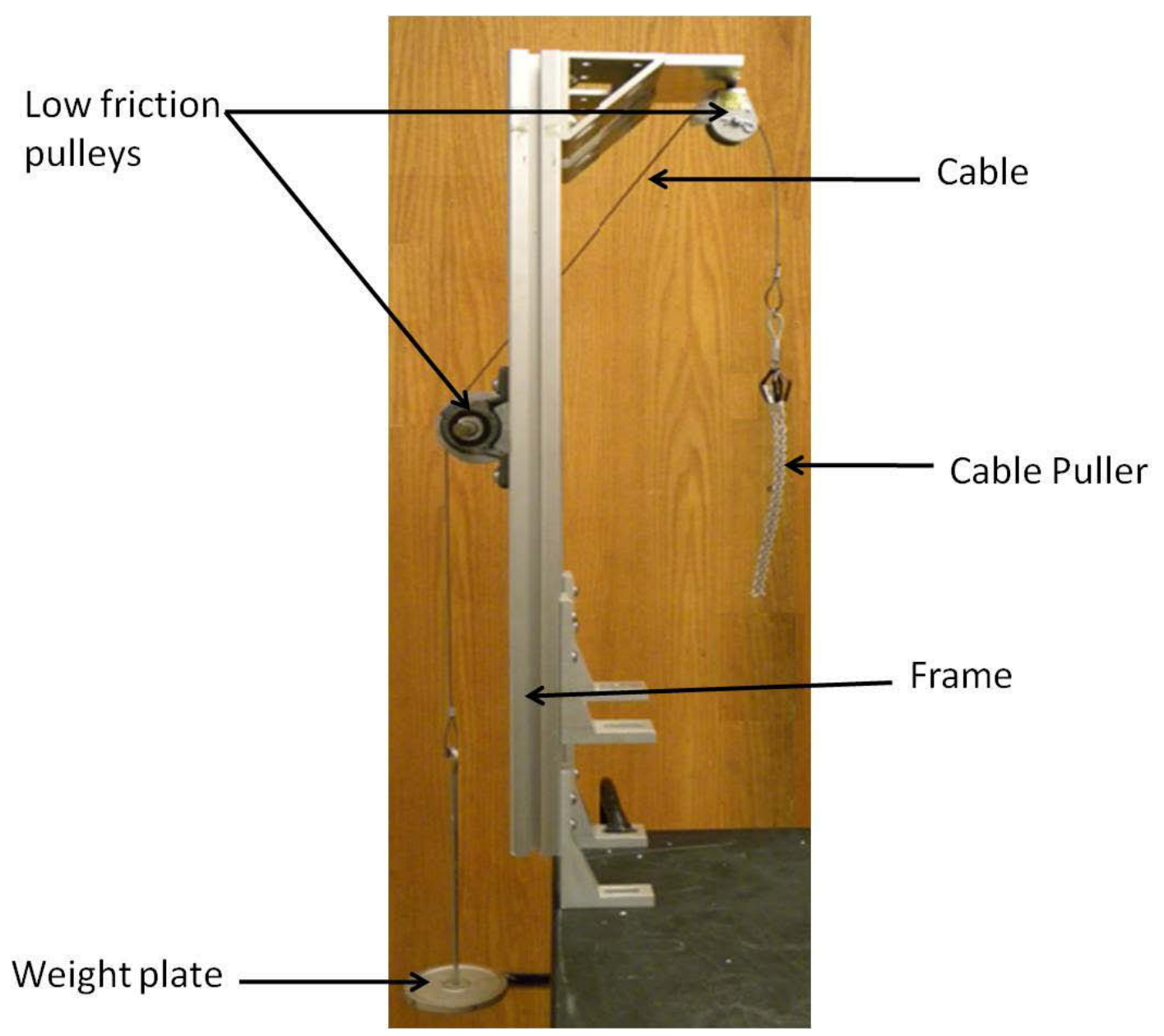

Figure 49: Achilles tendon loading system 


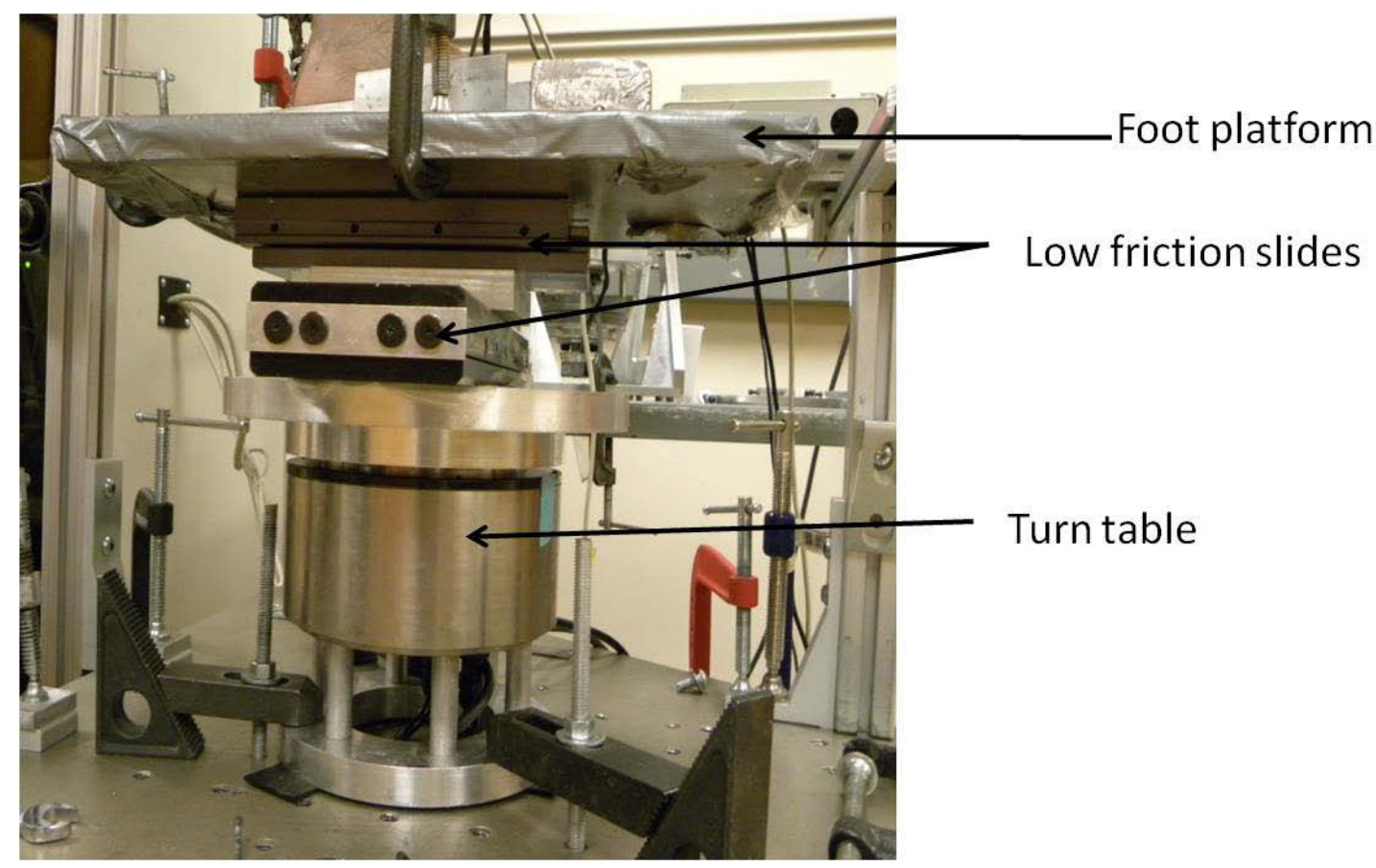

Figure 50: XY table mounted on the turn table 
When the specimen was mounted on the foot platform, any torque produced by the turn table was transmitted to the specimen through the XY table. The specimen was free to move in XY plane to reach a point of equilibrium as desired by the specimen and thus the XY table allowed creation of a less constrained foot model. This produced rotation about the natural center of rotation of each foot irrespective of other factors such as size of the foot. If desired, either the $\mathrm{X}$ and /or the $\mathrm{Y}$ slides could be locked using custom-designed plates to disable the $\mathrm{X}$ and/or $\mathrm{Y}$ degree of freedom.

\section{TIBIA MOUNTING FIXTURE}

\section{Objective}

To attach the tibia rigidly to the robot.

\section{Device Description and Action}

The tibia is the major weight bearing bone of the leg. It is roughly triangular in cross-section with one of the sides subcutaneous. The tibia is about 2 inches wide. The fibula is located on the lateral side of the tibia and is a slender bone up to 1 inch wide.

The CAD design of the device is shown in Figure 51. A semi-cylindrical pipe of 3 inches diameter and 1/8 inch thickness was welded to a $4 \times 4$ inch plate which was 1/4th inch thick. All these parts were made of steel. The plate had hole pattern to attach to the load cell.

As seen in Figure 52, this device was mounted on the load cell which was attached to the robot. The tibial end of the specimen which was potted (as described in the tissue preparation section) was placed in the semi-cylindrical pipe. Another semicylindrical pipe of the same diameter and thickness was fastened across the open half of the device using a hose clamp. The device was thus used to fix the tibial end of the specimen to the robot. 

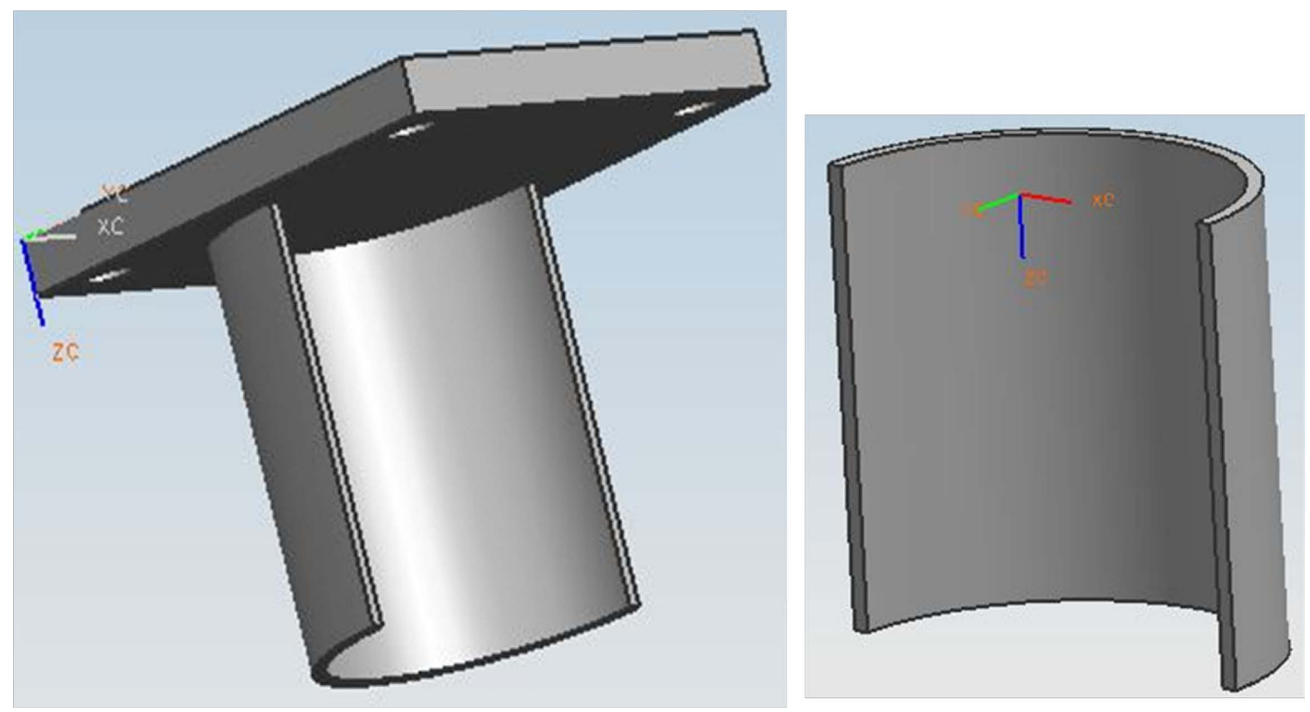

Figure 51: Tibia mounting device 


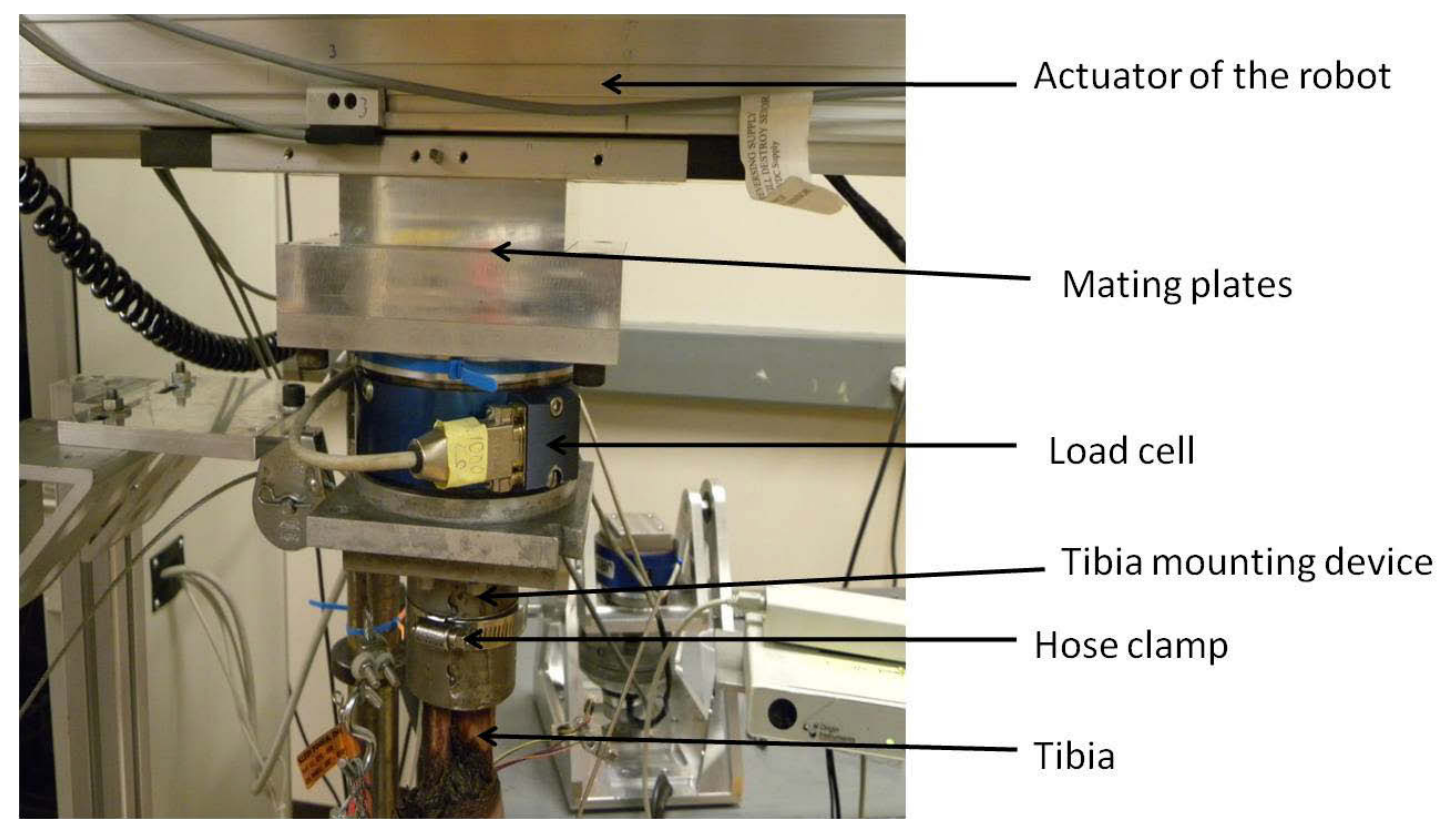

Figure 52: Tibia mounting device attached to load cell using mating plates

Note: The load cell is attached to the robot by appropriate fixtures. 


\section{APPENDIX B: STEP-BY-STEP DESCRIPTION OF EXPERIMENT}

\section{PREPARATION AND MOUNTING}

The specimen was prepared as described in the tissue preparation section and was mounted on the robot for testing. The entire assembly is shown in Figures 53 and 54.

A cylindrical tibia mounting device, whose design is described in Appendix A, was attached to load cell of the robot using mating plates. An XY table was constructed using two low friction slides and mounting plates as described in Appendix A. This XY table was mounted on a turntable which has been previously used.

After mounting the fixtures, the robot was turned on. The program designed for recreating the loading scenarios was started and the load cell zeroed. The specimen mounting command of the program was executed.

The foot specimen was placed on top of the XY table and the sides and front end of the specimen was constrained between aluminum bars as can be seen in the Figures 55 and 56. The tibial end of the specimen was now secured in the cylindrical mounting device using a hose clamp.

The specimen was thus completely constrained at the top/tibial end while it was relatively unconstrained at the sole/foot end since the foot was free to slide using the XY table. Finally the cable-puller, which enveloped the Achilles tendon, was attached to the tendon loading device, the design of which has been explained in Appendix A.

The Labview Vi was turned on and zeroed keeping targets in near zero positions. The Labview Vi was then turned off. The targets were mounted on the K-wires already inserted into the specimen using clamps as can be seen in the photograph. The Labview Vi recording was turned on and it started recording the target positions. Having mounted the specimen, the program was returned to the main menu and the four loading scenarios were executed.

\section{TESTING}

\section{Internal Rotation Sequential Test}

This test (Figure 30) involved application of internal rotation followed by compressive load. The Achilles tendon load of $350 \mathrm{~N}$ was applied. When this was done, the robot produced a 15 degrees turn using the turn table as long as the moment limit of $10 \mathrm{Nm}$ was not reached. For the internal rotation test, the turn was in clockwise direction for the left feet and counterclockwise direction for the right feet. At the end of rotation, the robot delivered $850 \mathrm{~N}$ of compression to the specimen. The compressive load and the 


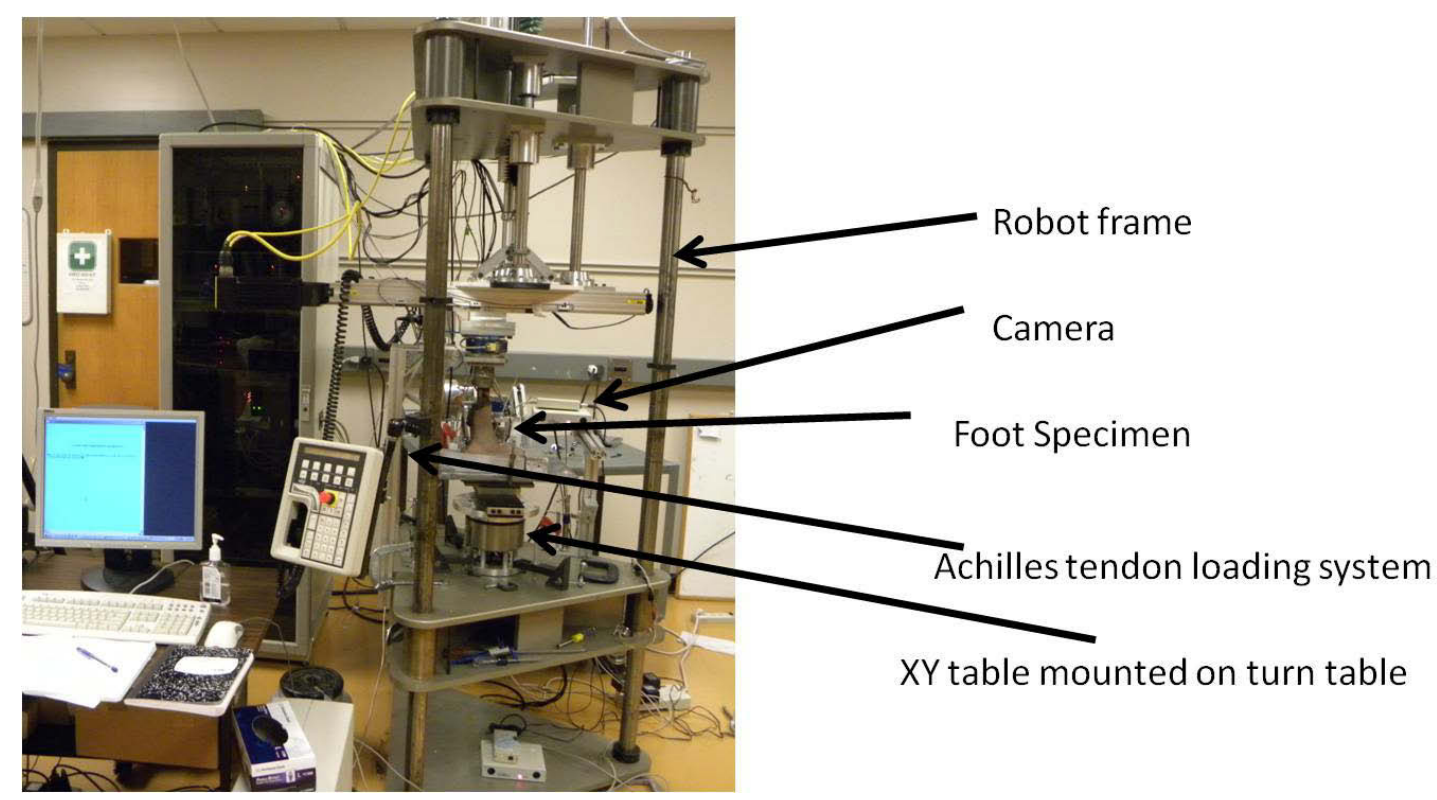

Figure 53: $\quad$ Entire assembly (side view) 


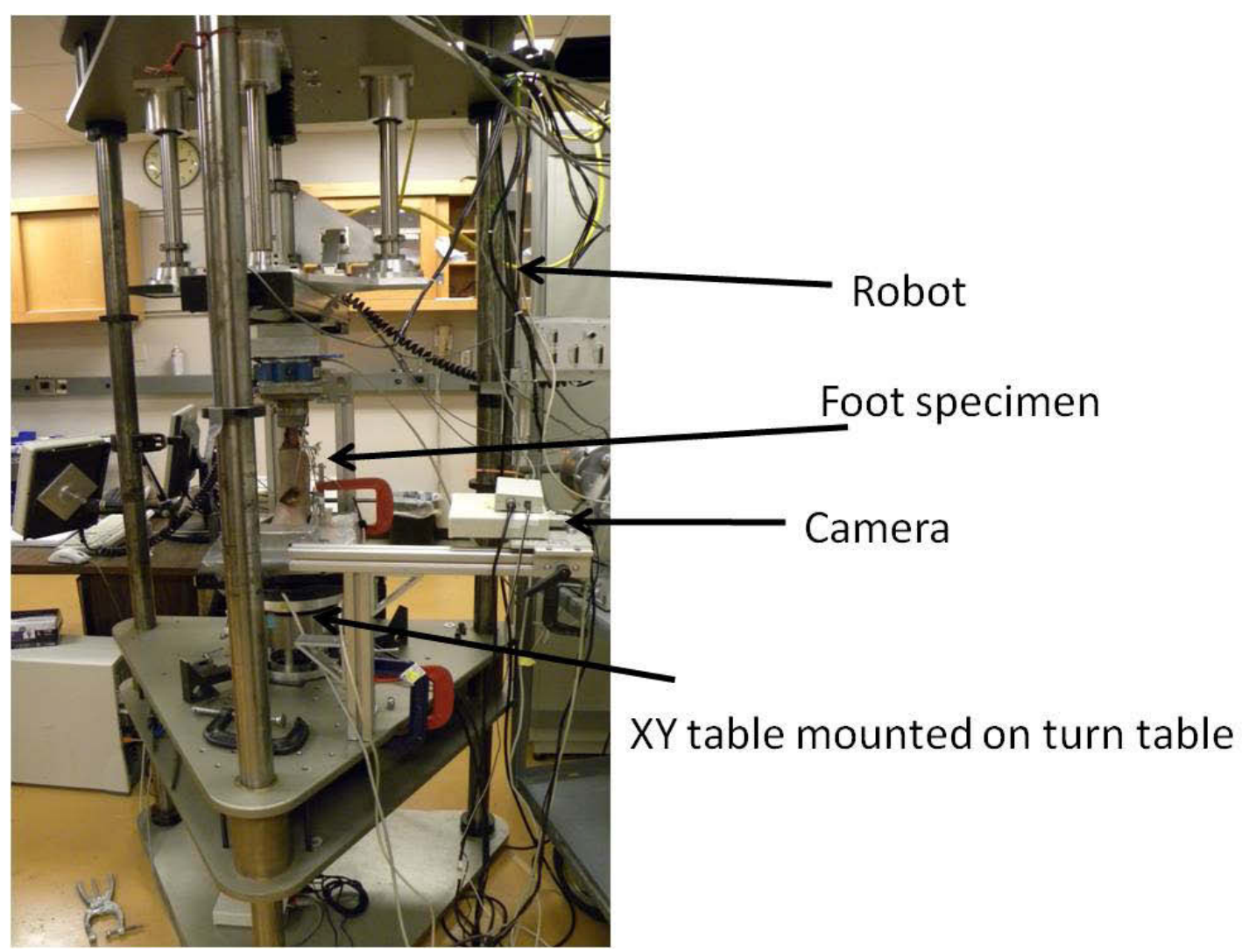

Figure 54: Entire assembly (front view) 


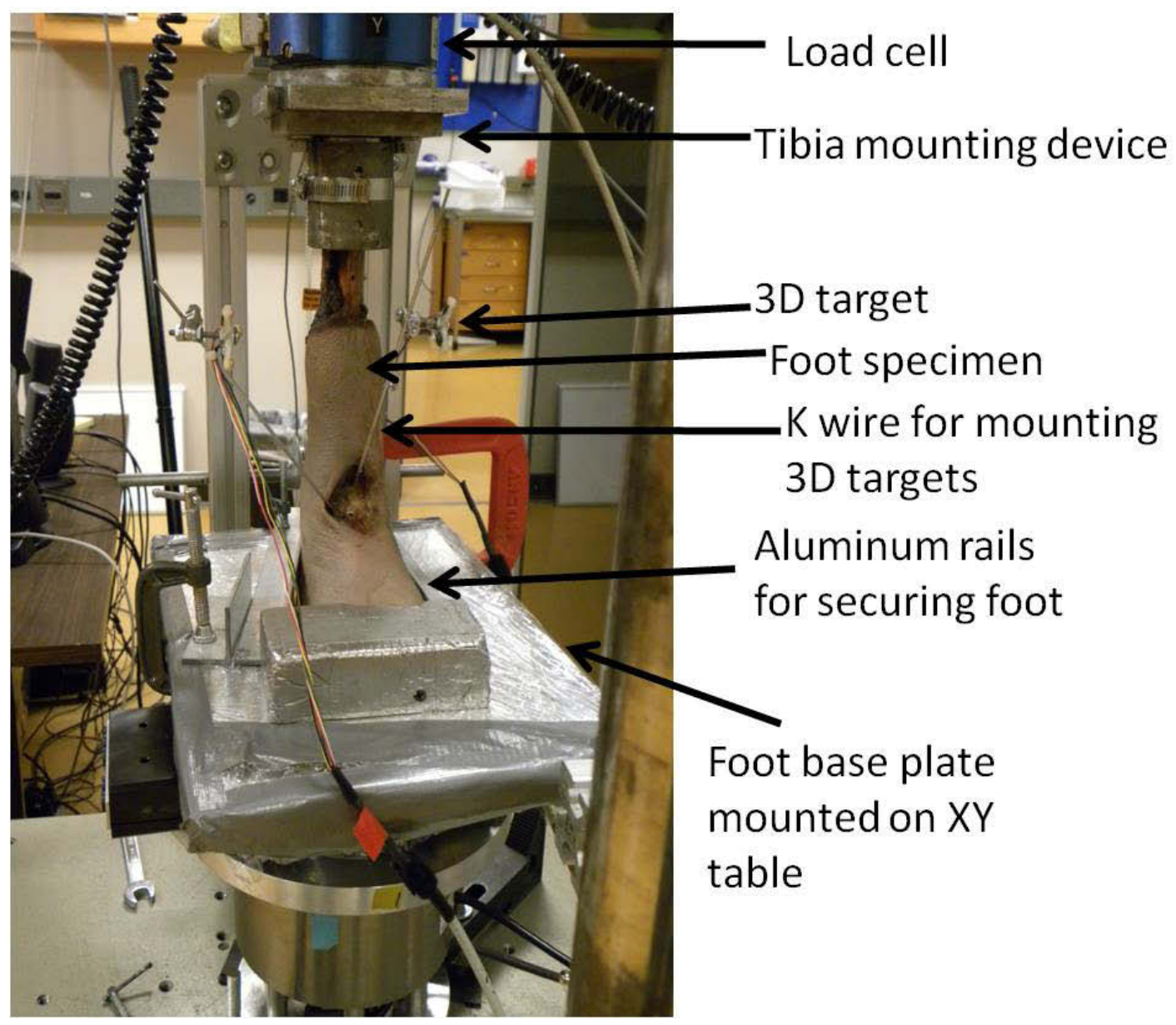

Figure 55: Mounting of foot (front view) 


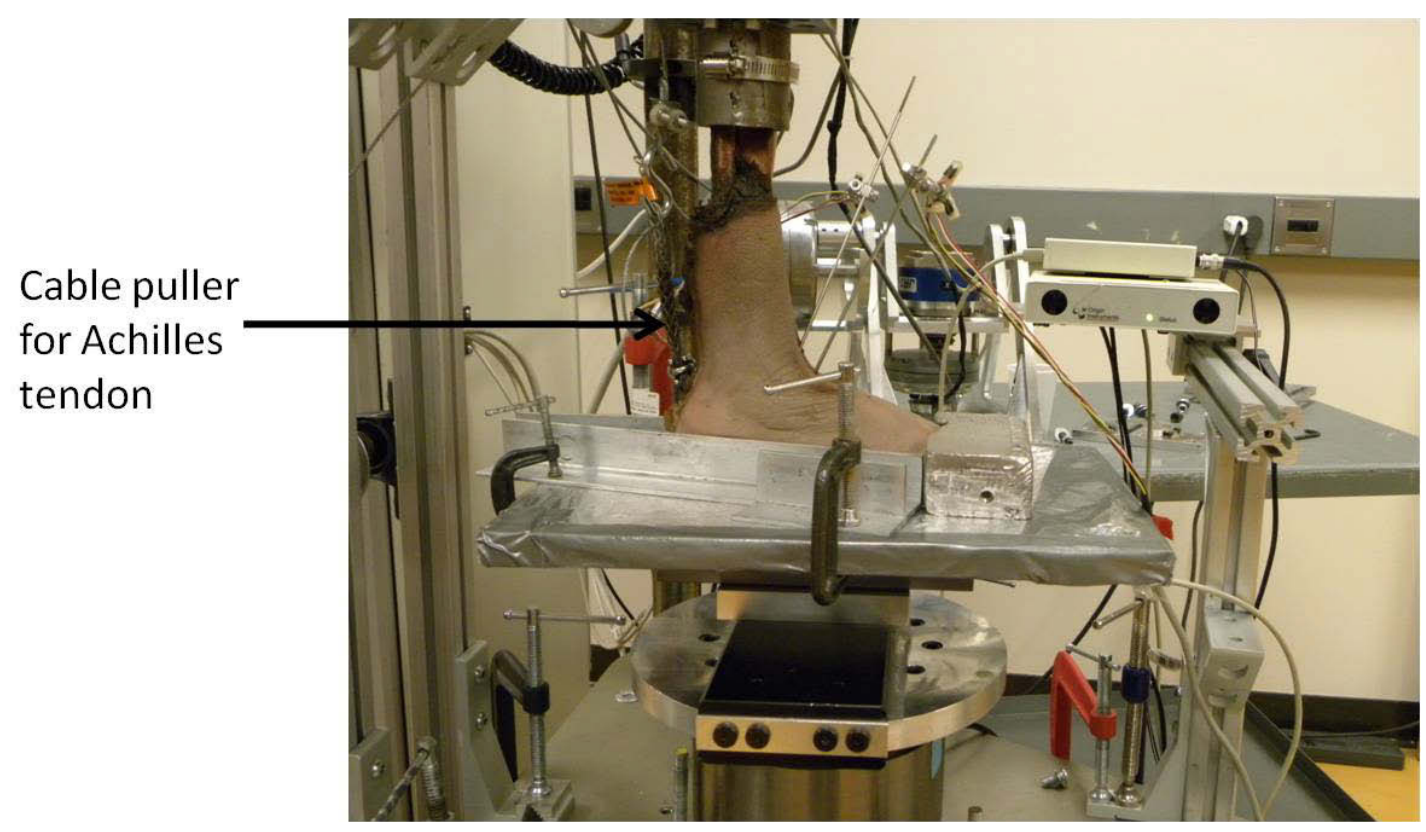

Figure 56: Mounting of foot (side view) 
rotation were removed followed by removal of the Achilles tendon load. This test also established the load and moment limits which were used to replay the loading in the internal rotation simultaneous test as described next.

\section{Internal Rotation Simultaneous Test}

This test involved simultaneous application of internal rotation and compressive load. The Achilles tendon load of $350 \mathrm{~N}$ was applied. When this was done, the robot produced a turn using the turn table and simultaneously applied compressive load until the limits as established during the sequential test were achieved. The test terminated automatically if either the load limit or the moment limit were exceeded. For this test, the turn was in clockwise direction for the left feet and counterclockwise direction for the right feet. When the desired load and rotation limits were reached, the compressive load and the rotation were removed followed by removal of the Achilles tendon load.

\section{External Rotation Sequential Test}

This test (Figure 31) involved application of external rotation followed by compressive load. The Achilles tendon load of $350 \mathrm{~N}$ was applied. When this was done, the robot produced a 15 degrees turn using the turn table as long as the moment limit of $10 \mathrm{NM}$ was not reached. For the external rotation test, the turn was in counterclockwise direction for the left feet and clockwise direction for the right feet. At the end of rotation, the robot delivered $850 \mathrm{~N}$ of compression to the specimen. The compressive load and the rotation were removed followed by removal of the Achilles tendon load. This test also established the load and moment limits which were used to replay the loading in the external rotation simultaneous test as described next.

\section{External Rotation Simultaneous Test}

This test involved simultaneous application of external rotation and compressive load. The Achilles tendon load of $350 \mathrm{~N}$ was applied. When this was done, the robot produced a turn using the turn table and simultaneously applied compressive load until the limits as established during the sequential test were achieved. The test terminated automatically if either the load limit or the moment limit were exceeded. For this test, the turn was in counterclockwise direction for the left feet and clockwise direction for the right feet. When the desired load and rotation limits were reached, the compressive load and the rotation were removed followed by removal of the Achilles tendon load.

At the end of each test, the Labview Vi was stopped and data was saved to a file. The robot data was also saved to a file. The files were named using a predefined naming convention which took into consideration the serial number of the specimen, side of the specimen (left or right), state of the specimen (harvested, plate plus one screw instrumentation or two screws instrumentation), and the test name (one of the four 
loading tests). All the data files were stored and later imported into Microsoft Excel ${ }^{\mathrm{TM}}$ and processed. 


\section{APPENDIX C: MEASUREMENTS OF OFFSETS}

\section{LOCALIZATION OF POINT J}

Motion of the targets attached to the talus and navicular bones was transformed into the talonavicular joint space. In order to do this, an arbitrary point $\mathrm{J}$ was defined in the joint space. The location of point $\mathrm{J}$ in the actual talus bone as compared with the radiographic views is shown in Figures 32, 33 and 34. The procedure used to define this point $\mathbf{J}$ in each specimen is described below.

A lateral radiograph of the foot was obtained as shown in Figure 57. In this radiograph, the convex margin of the talus was traced. The end points of this margin were joined to form a straight line. The midpoint of this line was identified and a perpendicular line was drawn from the midpoint to intersect the convex margin. The point of intersection was called point $J$. This process located point $J$ in the $X-Y$ plane.

In order to identify the depth of point $J$, i.e., the location of point $J$ along the $Z$ axis, a top view radiograph of the foot was obtained as shown in Figure 58. Again, the convex margin of the talus was traced, the end points of this margin were joined to form a straight line and a perpendicular line was drawn from the midpoint of this straight line to intersect the convex margin. The point of intersection was called point J. Thus, using two planar radiographs, the location of point $\mathrm{J}$ was defined in three dimensional space.

\section{EFFECT OF SURGERY ON LOCATION OF POINT J}

Point J was located on the head of the talus bone. During the surgical process, the head of the talus bone was not cut. The surgery involved removal of the articular cartilage only. Thus the position of point $\mathrm{J}$ was unaffected by the surgical process as seen in Figure 59 and was constant for the specimen throughout the study.

\section{MEASUREMENT OF OFFSET DISTANCES}

Point $\mathrm{J}$ was located at an offset from the targets inserted into the talus bone. To identify the exact offsets distance between point $\mathrm{J}$ and the targets, measurements were taken using calipers and radiographs.

\section{Measurement of Offset Distances Using Calipers}

As described in the tissue preparation section, K-wires were inserted into the talus and navicular bones at appropriate location in each bone. A target array was mounted on each K-wire using a clamp, which allowed the target to be adjusted as required. The target array mounted on the talus was thus offset at a distance from the K-wire in X, and 


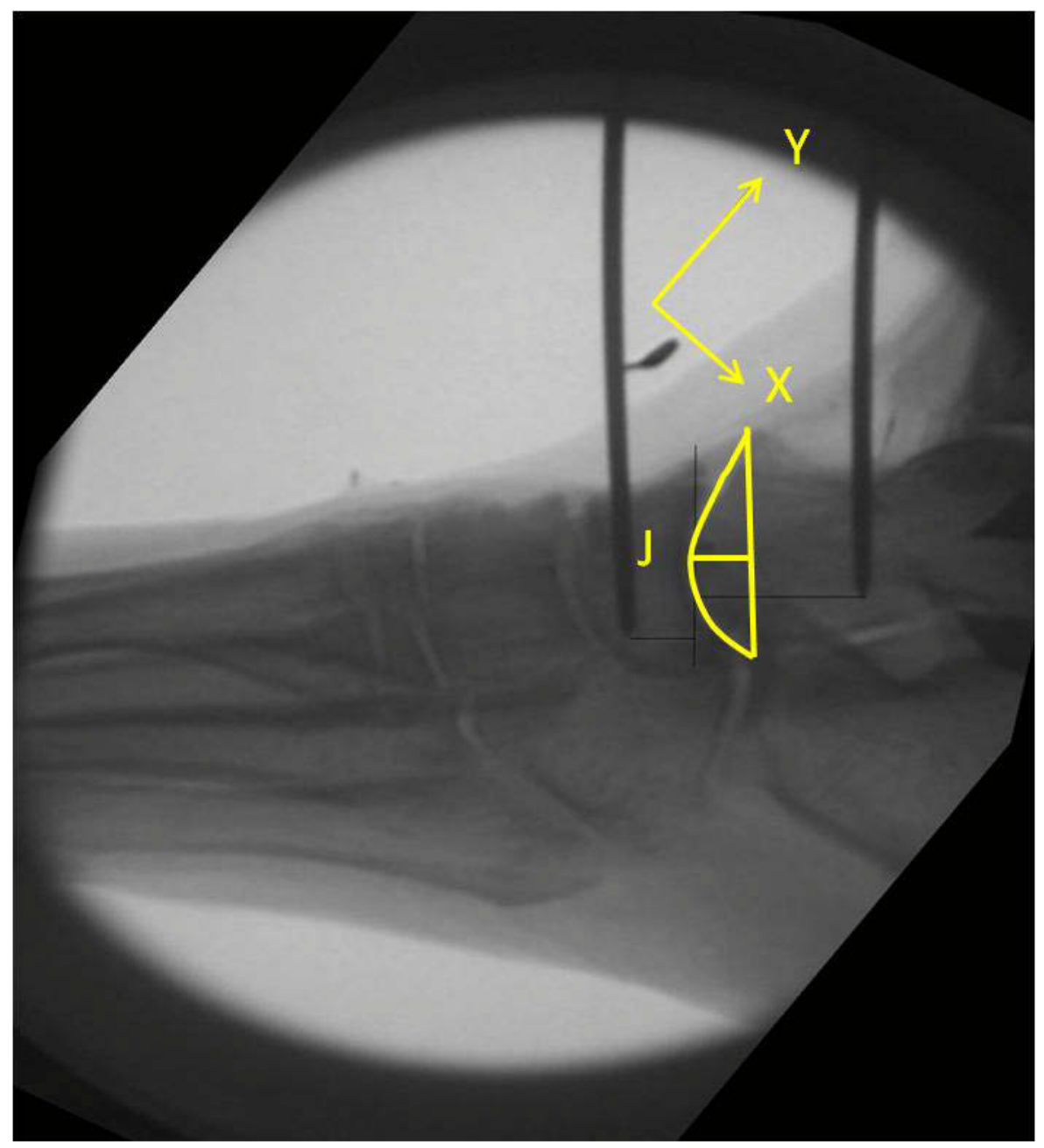

Figure 57: Lateral radiograph of foot showing XY location of point $J$

Note: The radiograph has been rotated. 


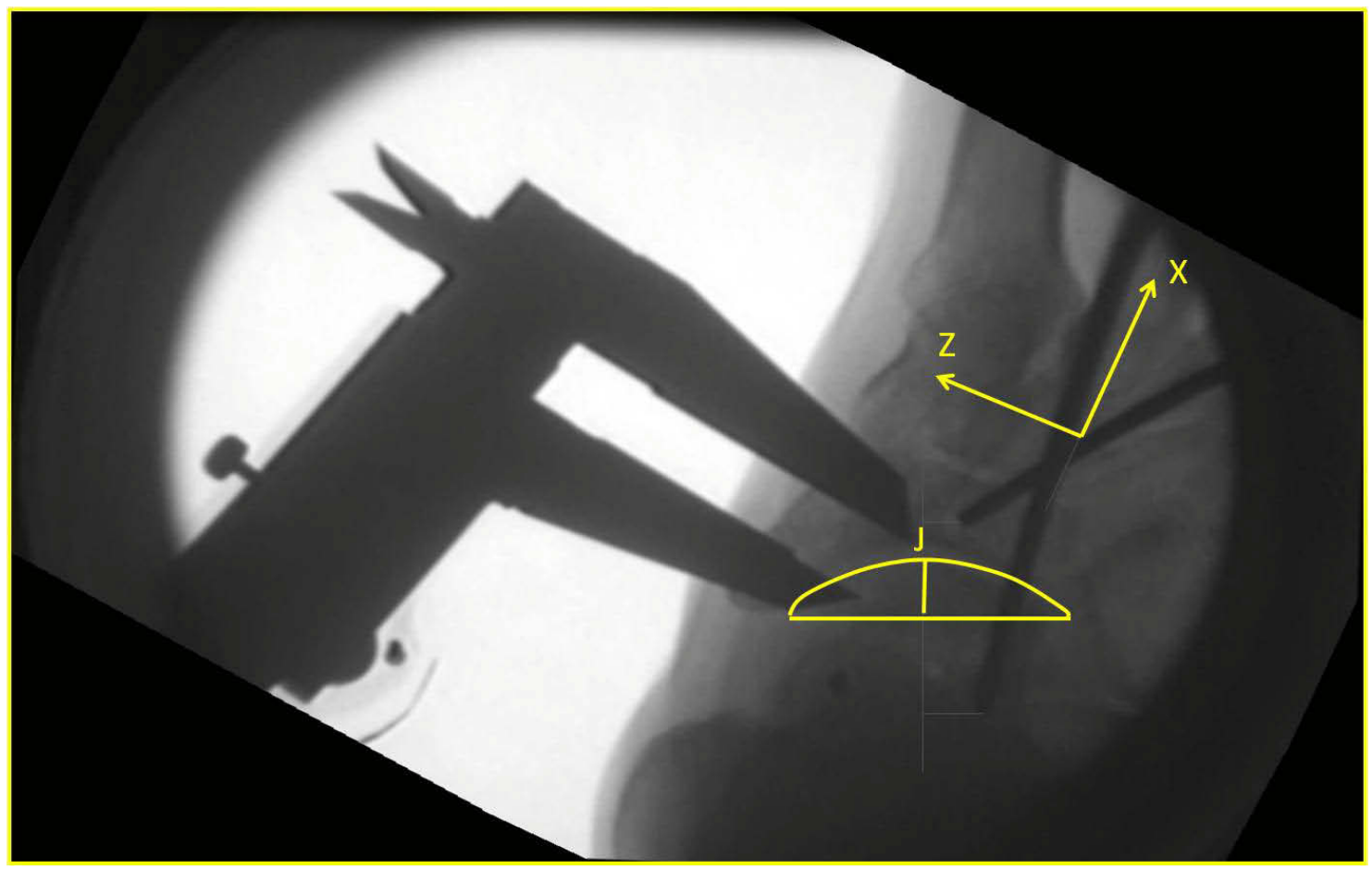

Figure 58: Top view of foot showing the depth of point $J$ and distance from lower end of K-wire

Note: The radiograph has been rotated. 


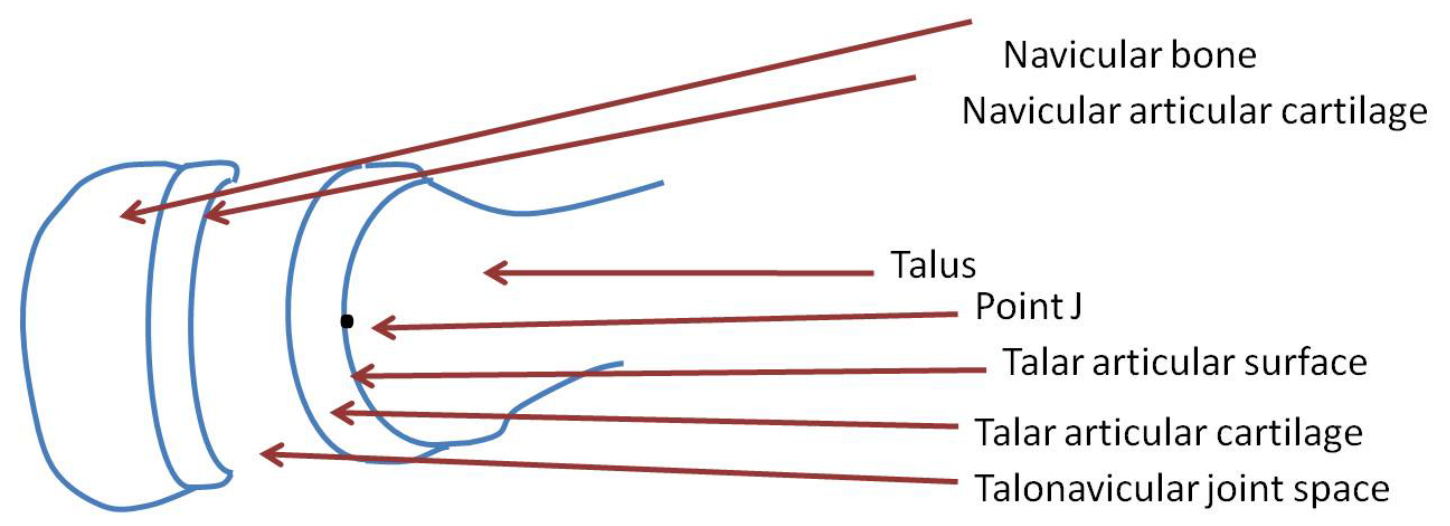

Preoperative condition of talonavicular joint

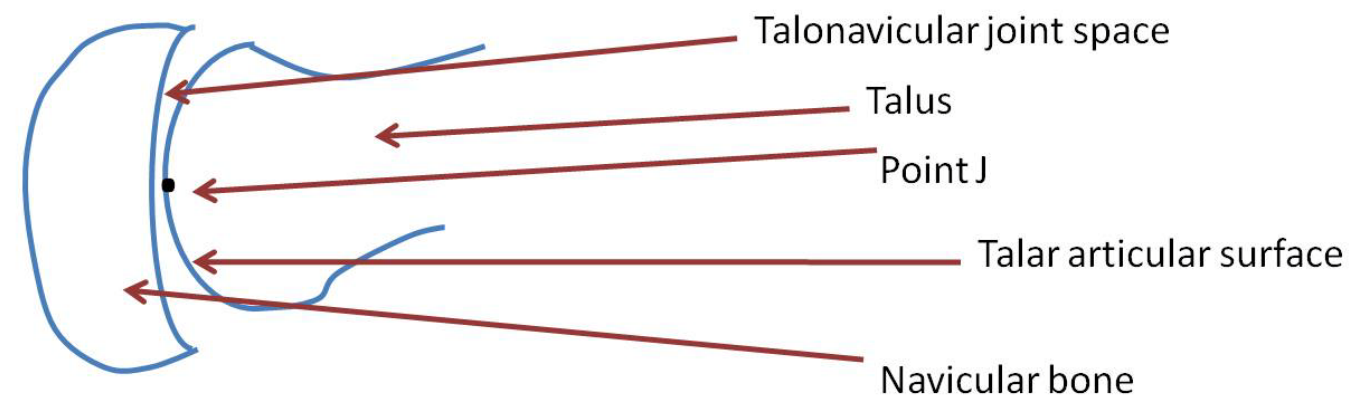

Postoperative condition of talonavicular joint- location of point $\mathrm{J}$ is unaffected by dissection of Talonavicular joint

Figure 59: Schematic representation of pre-operative and post-operative location of point $\mathbf{J}$ (lateral view) 
$\mathrm{Z}$ axis directions and was located above the lower end of the $\mathrm{K}$-wire in the $\mathrm{Y}$ axis direction as can be seen in Figures 60 and 61. The offset distances were measured using calipers as shown in Figures 60 and 61.

The distance from the midpoint of the target till the lower end of the K-wire was calculated by subtracting the distance between the target and upper end of the K-wire ( $h ")$ from the length of the K-wire ( $\left.\mathrm{g}^{\prime \prime}\right)$ which was constant for all K-wires. This was part of the y-offset called l", where,

$$
l^{\prime \prime}=g^{\prime \prime}-h^{\prime \prime}
$$

The offset distances between the midpoint of the target and the K-wire in the X and $\mathrm{Z}$ directions were directly measured using a caliper and were constant for all specimens. The part of the x-offset measured using calipers was called a" (Figure 60) and was fixed. It was $7.6 \mathrm{~mm}$ for all specimens.

The part of the z-offset measured using calipers was called p" (Figure 61) and was always $35 \mathrm{~mm}$ for the talus target for all specimens.

These measurements using calipers allowed determination of distance of the lower end of the $\mathrm{K}$ - wire called point $\mathrm{K}$ from the target array. In order to determine offset distance of point $\mathrm{J}$ from point $\mathrm{K}$, measurements were made using radiographs.

\section{Radiographic Measurement of Offset Distances}

A lateral radiographic view of the foot specimen was taken (Figure 57). A caliper set to $10 \mathrm{~mm}$ was placed in the view. This provided a reference distance. The image was imported into ImageJ software. Using the caliper as a standard, the scale of the image was adjusted.

The image was rotated until the talus K-wire was tilted at the exactly the same angle as that reported by the camera at time zero. With the image in this position, a line was drawn to join the widest points of the talar head. The angle of this line was the joint frame rotation angle about $Z$ axis called $\alpha_{1}$. A line was drawn perpendicular to this line and passing through its midpoint. The point where this line intersected the convex margin of the talar head was called point $J$. The lower end of the K-wire is called point $\mathrm{K}$.

The perpendicular distance between points $\mathrm{K}$ and $\mathrm{J}$ in the $\mathrm{X}$ axis (horizontal) direction was measured and called $b$. This was part of the $\mathrm{x}$-offset. The perpendicular distance between point $\mathrm{K}$ and $\mathrm{J}$ in the $\mathrm{Y}$ axis (vertical) direction was measured and called $\mathrm{m}$. This was part of the y-offset. A combined diagram showing all measurements (both radiographic and those made using calipers) in lateral view is provided as Figure 62. 


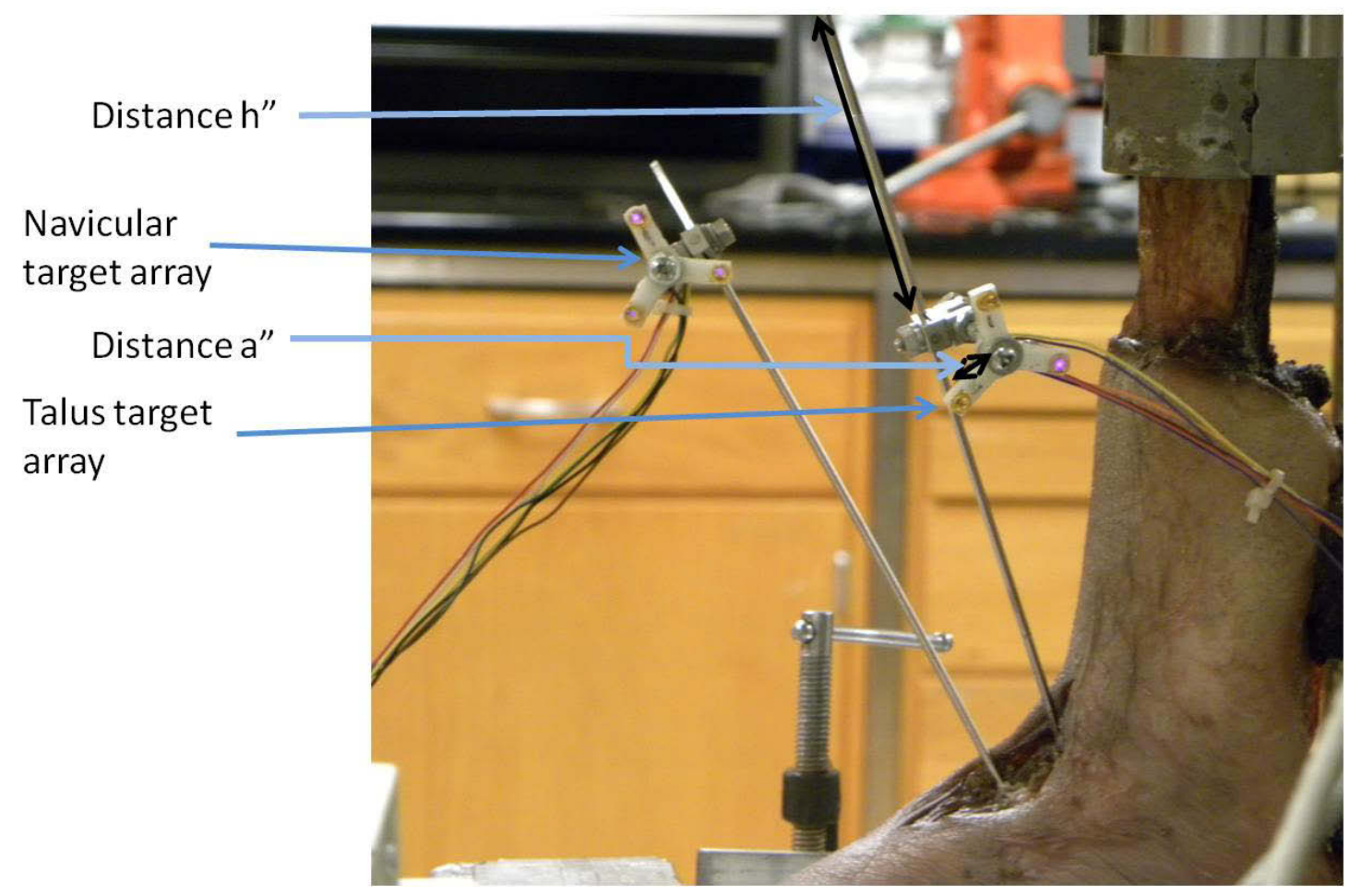

Figure 60: Target offset parameters (side view) 


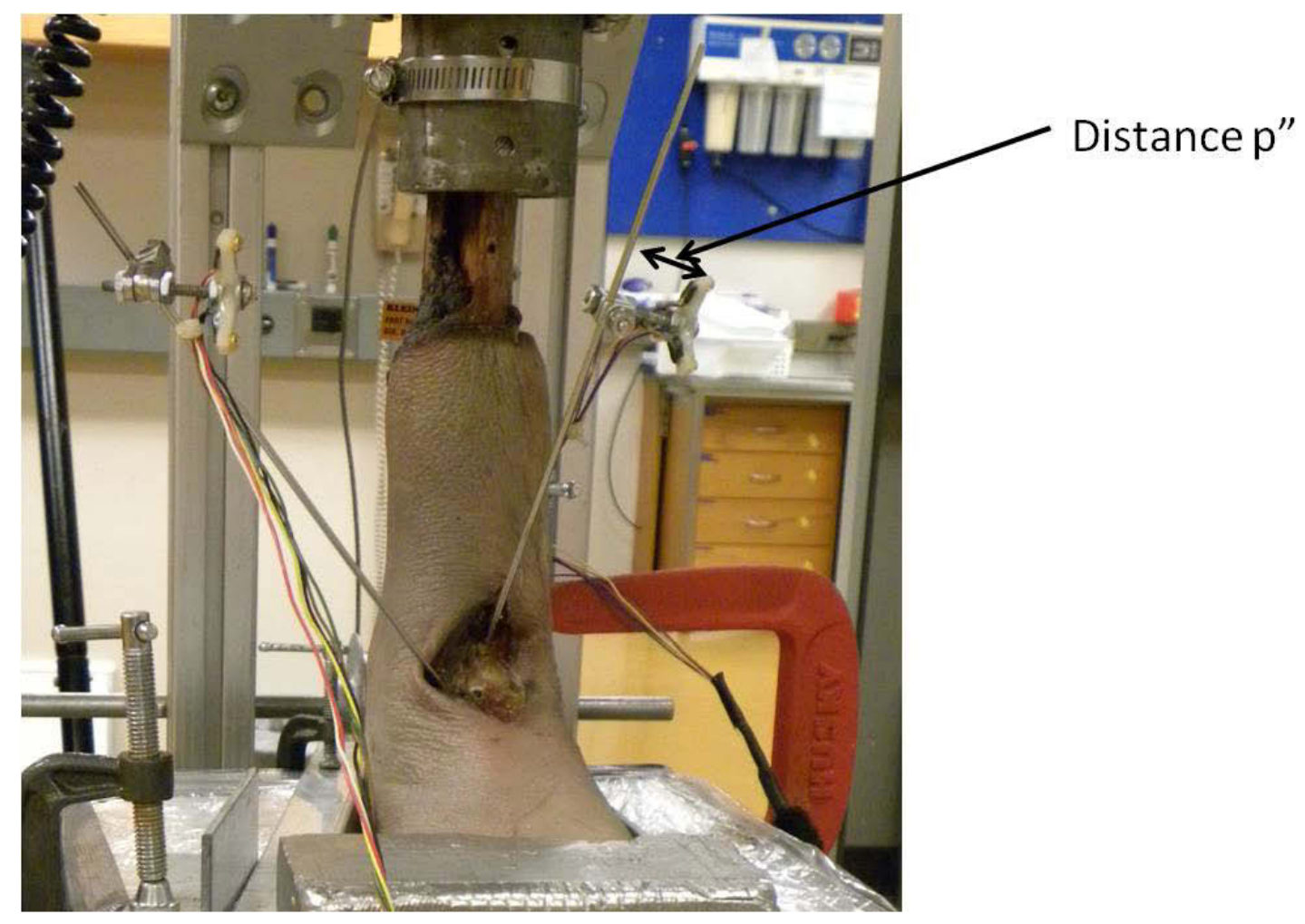

Figure 61: Target offset parameters (front view) 


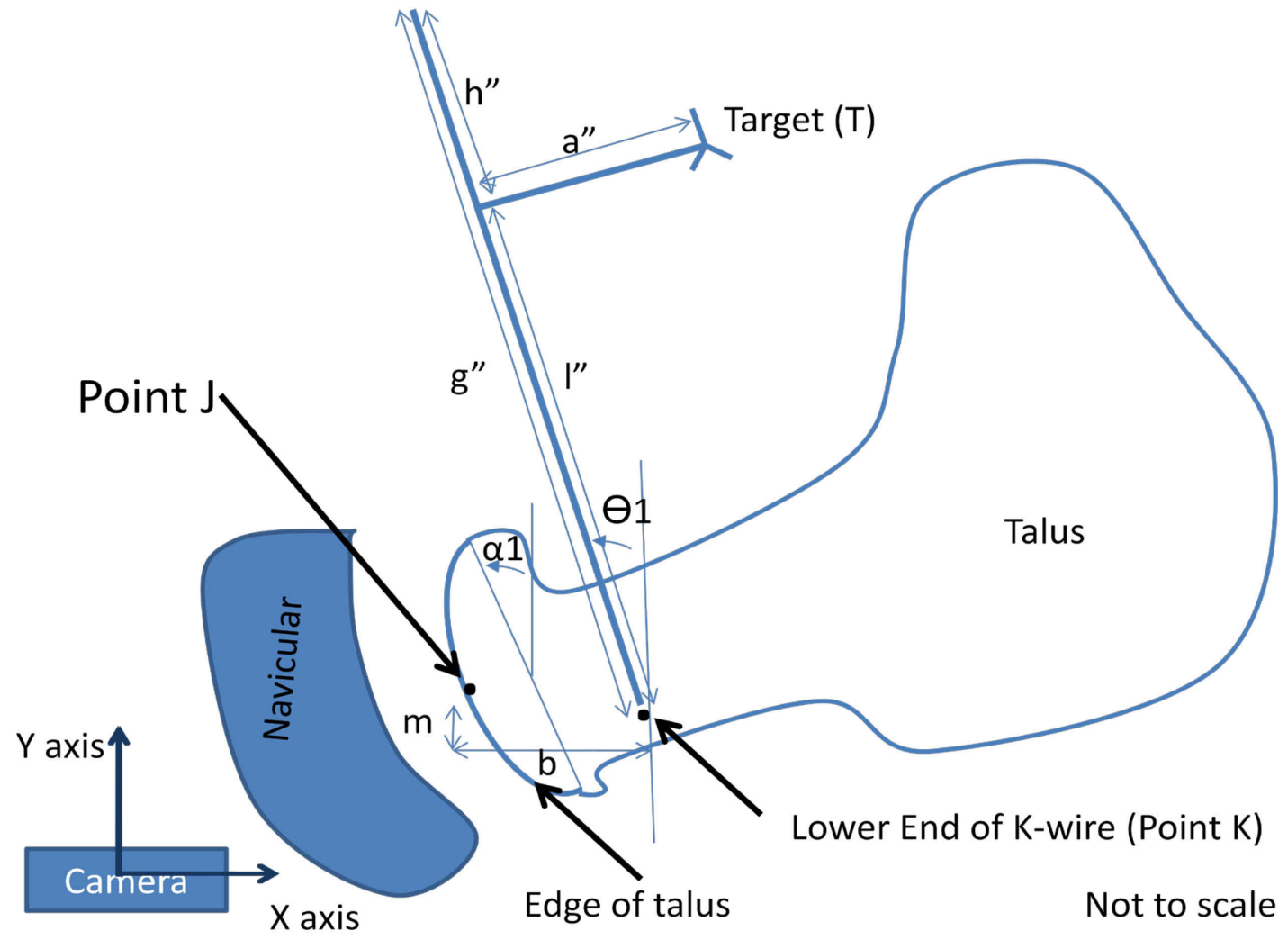

Figure 62: Diagrammatic representations of offset measurements (lateral view) 
To find the z-offset a similar procedure was followed. A top view radiograph of the foot specimen was obtained (Figure 58). A caliper set to $10 \mathrm{~mm}$ was placed in the view. This provided a reference distance. The image was imported into ImageJ. Using the caliper as a standard, the scale of the image was set.

A line was drawn which bisected the second metatarsal into two halves. The image was now rotated till this line was exactly vertical. A line was now drawn to join the widest points of the talar head. The angle of this line was the joint frame rotation angle about y axis called $\alpha_{2}$. A line was drawn perpendicular to this line and passing through its midpoint. The point where this line intersected the convex margin of the talar head was called point $\mathrm{J}$.

The perpendicular distance between this point $\mathrm{J}$ and the lower end of the $\mathrm{K}$-wire (K) was measured by drawing appropriate lines. This was the z-offset called q. This was part of the z-offset. A diagram showing all measurements in top view radiograph is provided as Figure 63. 


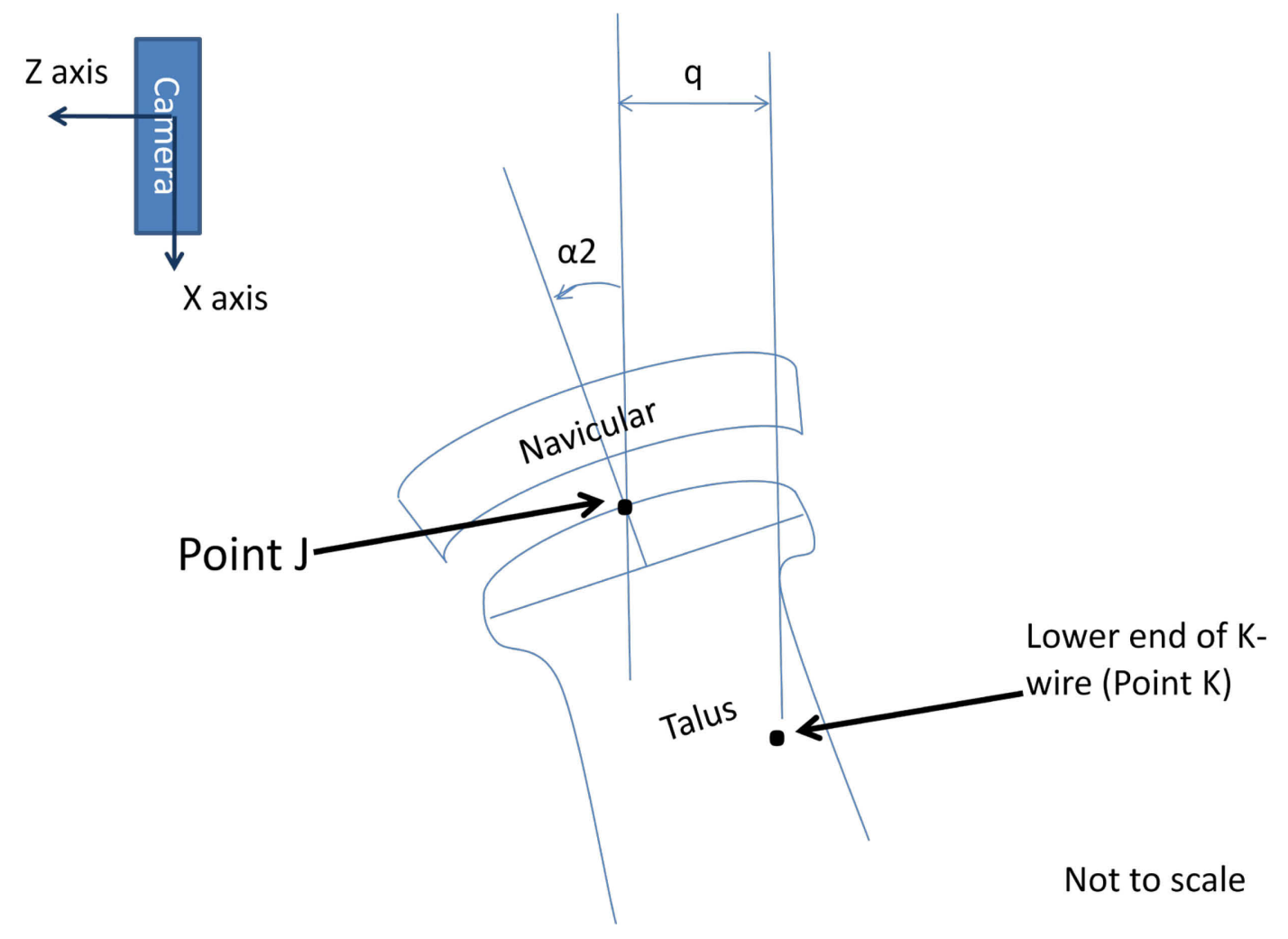

Figure 63: Diagrammatic representations of radiographic offset measurements (top view) 


\section{APPENDIX D: DERIVATION OF TRANSFORMATION MATRICES}

Consider a point $\mathrm{P}(\mathrm{x}, \mathrm{y})$ in the $\mathrm{XY}$ plane. The position of this point with respect to the origin can be represented by a vector $p$ which originates at the origin and ends at the point $\mathrm{P}$. This vector can be resolved into its components along two mutually perpendicular axes $\mathrm{X}$ and $\mathrm{Y}$. The components would be $\mathrm{x} i$ and $\mathrm{y} j$ where $i$ and $j$ are unit vectors along the $\mathrm{X}$ and $\mathrm{Y}$ axes respectively and $\mathrm{x}$ and $\mathrm{y}$ are the coordinates of point $\mathrm{P}$. This is illustrated by following diagram (Figure 64).

As shown in Figure 64, if the orientation of the XY axes i.e., the XY reference frame is changed by rotating it about the $\mathrm{Z}$ axis which is an axis passing through the origin and perpendicular to the XY plane, it can be observe that the vector $p$ remains unchanged. Thus the total magnitude of the vector remains unchanged. However, the magnitudes of the components change.

The magnitudes of the components along the new $\mathrm{X}^{\prime}$ and $\mathrm{Y}^{\prime}$ axes are given by:

$$
x^{\prime}=x^{*} \cos \Theta+y^{*} \sin \Theta
$$

and

$$
y^{\prime}=-x^{*} \sin \theta+y^{*} \cos \theta
$$

Where $\Theta$ is the angle of rotation.

The above transformation of coordinates due to rotation of the reference frame can be represented as a matrix as follows:

$$
\left[\begin{array}{l}
\mathrm{x}^{\prime} \\
\mathrm{y}^{\prime}
\end{array}\right]=\left[\begin{array}{cc}
\cos \Theta & \sin \Theta \\
-\sin \Theta & \cos \Theta
\end{array}\right]\left[\begin{array}{l}
\mathrm{x} \\
\mathrm{y}
\end{array}\right]
$$

Expanding to three dimensions - if the point $\mathrm{P}$ is a point in three dimensional space then it's coordinates $(\mathrm{x}, \mathrm{y}, \mathrm{z})$ can be transformed into new coordinates along the new $X^{\prime} Y^{\prime} Z^{\prime}$ reference frame using the matrix as follows:

$$
\left[\begin{array}{l}
x^{\prime} \\
y^{\prime} \\
z^{\prime}
\end{array}\right]=\left[\begin{array}{ccc}
\cos \Theta 1 & \sin \Theta 1 & 0 \\
-\sin \Theta 1 & \cos \Theta 1 & 0 \\
0 & 0 & 1
\end{array}\right]\left[\begin{array}{l}
\mathrm{x} \\
\mathrm{y} \\
\mathrm{z}
\end{array}\right]
$$

Where $\Theta_{1}$ is the angle of rotation about $\mathrm{Z}$ axis.

Similarly for rotation of reference frame about y axis we get:

$$
\left[\begin{array}{l}
\mathrm{x}^{\prime} \\
\mathrm{y}^{\prime} \\
\mathrm{z}^{\prime}
\end{array}\right]=\left[\begin{array}{ccc}
\cos \Theta 2 & 0 & -\sin \Theta 2 \\
0 & 1 & 0 \\
\sin \Theta 2 & 0 & \cos \Theta 2
\end{array}\right]\left[\begin{array}{l}
\mathrm{x} \\
\mathrm{y} \\
\mathrm{z}
\end{array}\right]
$$




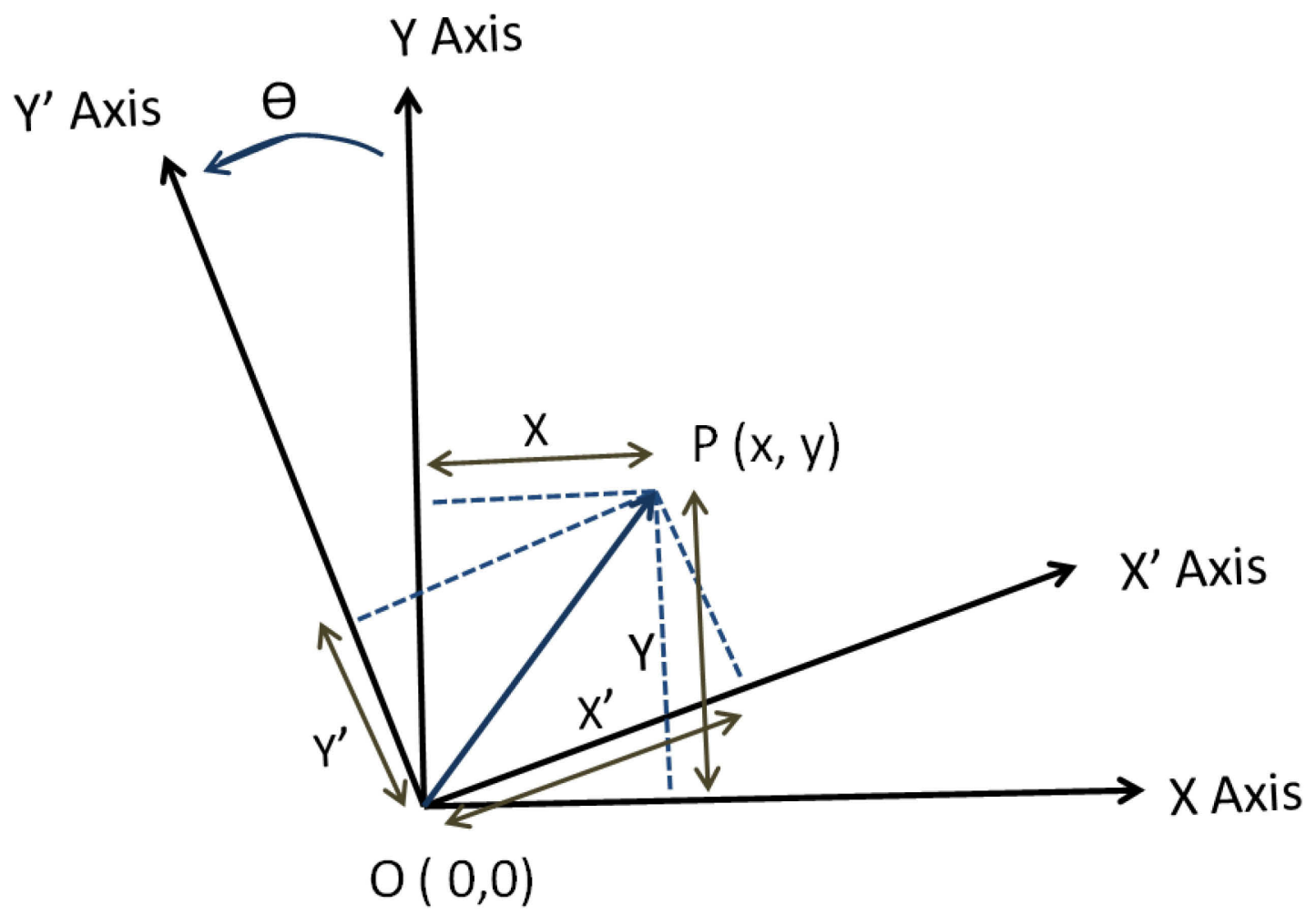

Figure 64: $\quad$ Rotation about $Z$ axis 
Where $\Theta_{2}$ is the angle of rotation about $\mathrm{Y}$ axis.

And for rotation of reference frame about $\mathrm{X}$ axis, we get:

$$
\left[\begin{array}{l}
\mathrm{x}^{\prime} \\
\mathrm{y}^{\prime} \\
\mathrm{z}^{\prime}
\end{array}\right]=\left[\begin{array}{ccc}
1 & 0 & 0 \\
0 & \cos \Theta 3 & \sin \Theta 3 \\
0 & -\sin \Theta 3 & \cos \Theta 3
\end{array}\right]\left[\begin{array}{l}
\mathrm{x} \\
\mathrm{y} \\
\mathrm{z}
\end{array}\right]
$$

Where $\Theta_{3}$ is the angle of rotation about $\mathrm{X}$ axis.

Thus the rotation matrices about $\mathrm{Z}, \mathrm{Y}$ and $\mathrm{X}$ axes are respectively:

$$
\begin{aligned}
& \mathrm{L}=\left[\begin{array}{ccc}
\cos \Theta 1 & \sin \Theta 1 & 0 \\
-\sin \Theta 1 & \cos \Theta 1 & 0 \\
0 & 0 & 1
\end{array}\right] \\
& \mathrm{M}=\left[\begin{array}{ccc}
\cos \Theta 2 & 0 & -\sin \Theta 2 \\
0 & 1 & 0 \\
\sin \Theta 2 & 0 & \cos \Theta 2
\end{array}\right] \\
& \mathrm{N}=\left[\begin{array}{ccc}
1 & 0 & 0 \\
0 & \cos \Theta 3 & \sin \Theta 3 \\
0 & -\sin \Theta 3 & \cos \Theta 3
\end{array}\right]
\end{aligned}
$$

If we perform sequential rotation of $X Y Z$ frame first about $\mathrm{Z}$ axis, followed by $\mathrm{Y}$ axis, followed by $X$ axis, the resultant transformation matrix (A) would be a multiplication of the three matrices in that sequence:

$$
\mathrm{A}=\mathrm{N} * \mathrm{M} * \mathrm{~L}
$$

Substituting the values, we get:

$$
A=\left[\begin{array}{ccc}
\mathrm{C} 1 \mathrm{C} 2 & \mathrm{~S} 1 \mathrm{C} 2 & -\mathrm{S} 2 \\
-\mathrm{S} 1 \mathrm{C} 3+\mathrm{C} 1 \mathrm{~S} 2 \mathrm{~S} 3 & \mathrm{C} 1 \mathrm{C} 3+\mathrm{S} 1 \mathrm{~S} 2 \mathrm{~S} 3 & \mathrm{C} 2 \mathrm{~S} 3 \\
\mathrm{~S} 1 \mathrm{~S} 3+\mathrm{C} 1 \mathrm{~S} 2 \mathrm{C} 3 & -\mathrm{C} 1 \mathrm{~S} 3+\mathrm{S} 1 \mathrm{~S} 2 \mathrm{C} 3 & \mathrm{C} 2 \mathrm{C} 3
\end{array}\right]
$$

Where,

$$
\begin{array}{lll}
\mathrm{C} 1 & = & \cos \Theta_{1} \\
\mathrm{C} 2 & = & \cos \Theta_{2} \\
\mathrm{C} 3 & = & \cos \Theta_{3} \\
\mathrm{~S} 1 & = & \sin \Theta_{1} \\
\mathrm{~S} 2 & = & \sin \Theta_{2}
\end{array}
$$




$$
\mathrm{S} 3=\sin \Theta_{3}
$$

This is the matrix to go from an upright/neutral reference frame into any rotated frame of reference. Thus, if we know the coordinates of a point $\mathrm{P}$ in the upright frame $(\mathrm{x}, \mathrm{y}, \mathrm{z})$, we can get the coordinates in the rotated frame using:

$$
\left[\begin{array}{l}
x^{\prime} \\
y^{\prime} \\
z^{\prime}
\end{array}\right]=\left[\begin{array}{ccc}
\mathrm{C} 1 \mathrm{C} 2 & \mathrm{~S} 1 \mathrm{C} 2 & -\mathrm{S} 2 \\
-\mathrm{S} 1 \mathrm{C} 3+\mathrm{C} 1 \mathrm{~S} 2 \mathrm{~S} 3 & \mathrm{C} 1 \mathrm{C} 3+\mathrm{S} 1 \mathrm{~S} 2 \mathrm{~S} 3 & \mathrm{C} 2 \mathrm{~S} 3 \\
\mathrm{~S} 1 \mathrm{~S} 3+\mathrm{C} 1 \mathrm{~S} 2 \mathrm{C} 3 & -\mathrm{C} 1 \mathrm{~S} 3+\mathrm{S} 1 \mathrm{~S} 2 \mathrm{C} 3 & \mathrm{C} 2 \mathrm{C} 3
\end{array}\right]\left[\begin{array}{l}
\mathrm{x} \\
\mathrm{y} \\
\mathrm{z}
\end{array}\right]
$$

Similarly, if we know the coordinates in the rotated frame of reference, we can get the coordinates in the neutral/upright frame of reference using matrix B, where $\mathrm{B}$ is given by multiplying the inverse of each rotation matrix in the reverse sequence.

$$
\begin{aligned}
& \mathrm{B}=\mathrm{L}^{-1} * \mathrm{M}^{-1} * \mathrm{~N}^{-1} \\
& B=\left[\begin{array}{ccc}
\mathrm{C} 1 \mathrm{C} 2 & \mathrm{C} 1 \mathrm{~S} 2 \mathrm{~S} 3-\mathrm{S} 1 \mathrm{C} 3 & \mathrm{C} 1 \mathrm{~S} 2 \mathrm{C} 3+\mathrm{S} 1 \mathrm{~S} 3 \\
\mathrm{~S} 1 \mathrm{C} 2 & \mathrm{~S} 1 \mathrm{~S} 2 \mathrm{~S} 3+\mathrm{C} 1 \mathrm{C} 3 & \mathrm{~S} 1 \mathrm{~S} 2 \mathrm{C} 3-\mathrm{C} 1 \mathrm{~S} 3 \\
-\mathrm{S} 2 & \mathrm{C} 2 \mathrm{~S} 3 & \mathrm{C} 2 \mathrm{C} 3
\end{array}\right]
\end{aligned}
$$

Where,

$$
\begin{array}{lll}
\mathrm{C} 1 & = & \cos \Theta_{1} \\
\mathrm{C} 2 & = & \cos \Theta_{2} \\
\mathrm{C} 3 & = & \cos \Theta_{3} \\
\mathrm{~S} 1 & = & \sin \Theta_{1} \\
\mathrm{~S} 2 & = & \sin \theta_{2} \\
\mathrm{~S} 3 & = & \sin \Theta_{3}
\end{array}
$$

If the rotation is about $\mathrm{Y}$ axis followed by $\mathrm{Z}$ axis and finally followed by $\mathrm{X}$ axis, then the transformation matrix would be:

$$
\begin{array}{ccc}
\mathrm{C}=\mathrm{N} * \mathrm{~L} * \mathrm{M} & \\
\mathrm{C}=\left[\begin{array}{ccc}
\mathrm{C} 1 \mathrm{C} 2 & \mathrm{~S} 1 & -\mathrm{C} 1 \mathrm{~S} 2 \\
-\mathrm{S} 1 \mathrm{C} 2 \mathrm{C} 3+\mathrm{S} 2 \mathrm{~S} 3 & \mathrm{C} 1 \mathrm{C} 3 & \mathrm{~S} 1 \mathrm{~S} 2 \mathrm{C} 3+\mathrm{C} 2 \mathrm{~S} 3 \\
\mathrm{~S} 1 \mathrm{C} 2 \mathrm{~S} 3+\mathrm{S} 2 \mathrm{C} 3 & -\mathrm{C} 2 \mathrm{~S} 3 & -\mathrm{S} 1 \mathrm{~S} 2 \mathrm{~S} 3+\mathrm{C} 2 \mathrm{C} 3
\end{array}\right]
\end{array}
$$




\section{APPENDIX E: DATA PROCESSING}

\section{DATA PROCESSING (TRANSLATION)}

Consider the target as a point $\mathrm{T}$ in three dimensional space. From the Labview $\mathrm{Vi}$, which processes the camera data, we get the coordinates and the rotations of the $\operatorname{target}$ as $\mathrm{x}, \mathrm{y}, \mathrm{z}$ and rotations as about $\mathrm{Z}$ axis, about $\mathrm{Y}$ axis and about $-\mathrm{X}$ axis in that sequence.

The raw data obtained from the Labview Vi was imported into Microsoft Excel ${ }^{\mathrm{TM}}$ and all further operations were done using Microsoft Excel ${ }^{\mathrm{TM}}$. The rotation rate reported by the Labview Vi follows a left hand coordinate system. To convert it into a right hand system, the about - $X$ axis angle was multiplied by "-1." This gave us the angles about $Z$ axis, about $Y$ axis and about $X$ axis in that sequence. We will call the rotations as $\Theta_{1}, \Theta_{2}$, and $\Theta_{3}$, respectively. The Labview Vi is based on Euler angles and the sequence of rotations was important.

Our objective was to transform the target data from the talus and navicular target to a point $\mathrm{J}$ and compare the results between the two results. If there is no relative motion between the two bones, then point $\mathrm{J}$ will have the same coordinates whether they are derived from talus or the navicular targets.

To achieve this, we first assume the existence of point J. Then, we use the Talus and Navicular coordinates at time zero and the offset measurements (described in Appendix C) to determine the location of point J at time zero (Step 1 to Step 4). We use this location to define vectors from each target array to point $J$ in the camera frame (Step 5) and in the target frame (Steps 6 and 7). We use these vectors from Step 7 and the instantaneous coordinates of talus and navicular targets obtained from Labview to find the instantaneous coordinates of point $J$ for each time point (Steps 8 and 9). We take the difference between the coordinates of point $\mathrm{J}$ calculated using talus and navicular target data to determine the relative translations between the two bones (Step 10). Finally this relative translation is transformed into the joint reference frame to determine the relative translation between the bones in the joint reference frame (Steps 11 and 12). Thus we will be using three frames of reference to process data obtained from each target array.

1. Camera reference frame represented by $\mathrm{X}, \mathrm{Y}$, and $\mathrm{Z}$ axes

2. Joint reference frame represented by $X^{\prime}, Y^{\prime}$ and $Z^{\prime}$ axes.

3. Target reference frames represented by $\mathrm{X}^{\prime \prime}, \mathrm{Y}^{\prime \prime}$ and $\mathrm{Zn}$ axes

We assume there exists a point $\mathrm{J}$ and we will describe all the motion occurring at point $\mathrm{J}$. The process of defining and localizing point $\mathrm{J}$ has been described in Appendix $\mathrm{C}$. 


\section{Step 1}

Now, consider the talus target. As seen in Figure 65, we assume the target midpoint $(\mathrm{T})$ to be the origin with coordinates $(0,0,0)$ and rotations $(0,0,0)$. We assume that the axes of the target are aligned with the camera axes. This target is mounted on the $\mathrm{K}$-wire using clamps and hence it is offset from the $\mathrm{K}$-wire as previously described. The lower end of the $\mathrm{K}$-wire is called point $\mathrm{K}$. With respect to target midpoint $\mathrm{T}$, the coordinates of $\mathrm{K}$ are (-a", -1", -p"). The measurements of $\mathrm{a}^{\prime \prime}, 1 "$ and $\mathrm{p}$ " has been previously described.

\section{Step 2}

As seen in Figure 66, at time zero, the target is not perfectly aligned with the camera, i.e., it has some rotations with respect to camera axes. Thus we need to transform the coordinates of $\mathrm{K}$ from the rotated frame of the target to the upright camera frame. For this we use the transformation matrix B, which has been derived in Appendix D.

$$
\left[\begin{array}{l}
a \\
l \\
p
\end{array}\right]=\left[\begin{array}{ccc}
C 1 C 2 & C 1 S 2 S 3-S 1 C 3 & C 1 S 2 C 3+S 1 S 3 \\
S 1 C 2 & S 1 S 2 S 3+C 1 C 3 & S 1 S 2 C 3-C 1 S 3 \\
-S 2 & C 2 S 3 & C 2 C 3
\end{array}\right]\left[\begin{array}{c}
-a^{\prime \prime} \\
-l^{\prime \prime} \\
-p^{\prime \prime}
\end{array}\right]
$$

Thus we get the coordinates of $\mathrm{K}$ in camera frame as $(\mathrm{a}, 1, \mathrm{p})$.

\section{Step 3}

We know from radiographic data that, $\mathrm{K}$ is offset from $\mathrm{J}$ by $\mathrm{b}, \mathrm{m}$ and $\mathrm{q}$ in the $\mathrm{X}, \mathrm{Y}$ and $\mathrm{Z}$ axis directions respectively. Thus the coordinates of $\mathrm{J}$ with respect to $\mathrm{T}$ as the origin of the target frame are (a-b, l-m, p-q). For sake of simplicity we will call these (xj"', yj"', zj"'). These are the coordinates of point $\mathrm{J}$ in the upright frame, as seen in Figure 67 with $\mathrm{T}$ as the origin.

$$
\left[\begin{array}{l}
x j^{\prime \prime \prime} \\
y j^{\prime \prime \prime} \\
z j^{\prime \prime \prime}
\end{array}\right]=\left[\begin{array}{l}
a \\
l \\
p
\end{array}\right]-\left[\begin{array}{c}
b \\
m \\
q
\end{array}\right]
$$

\section{Step 4}

As seen in Figure 68, the point $\mathrm{T}$ is not the actual origin but it is located at finite coordinates with respect to the camera origin. These coordinates are represented by the following matrix: 


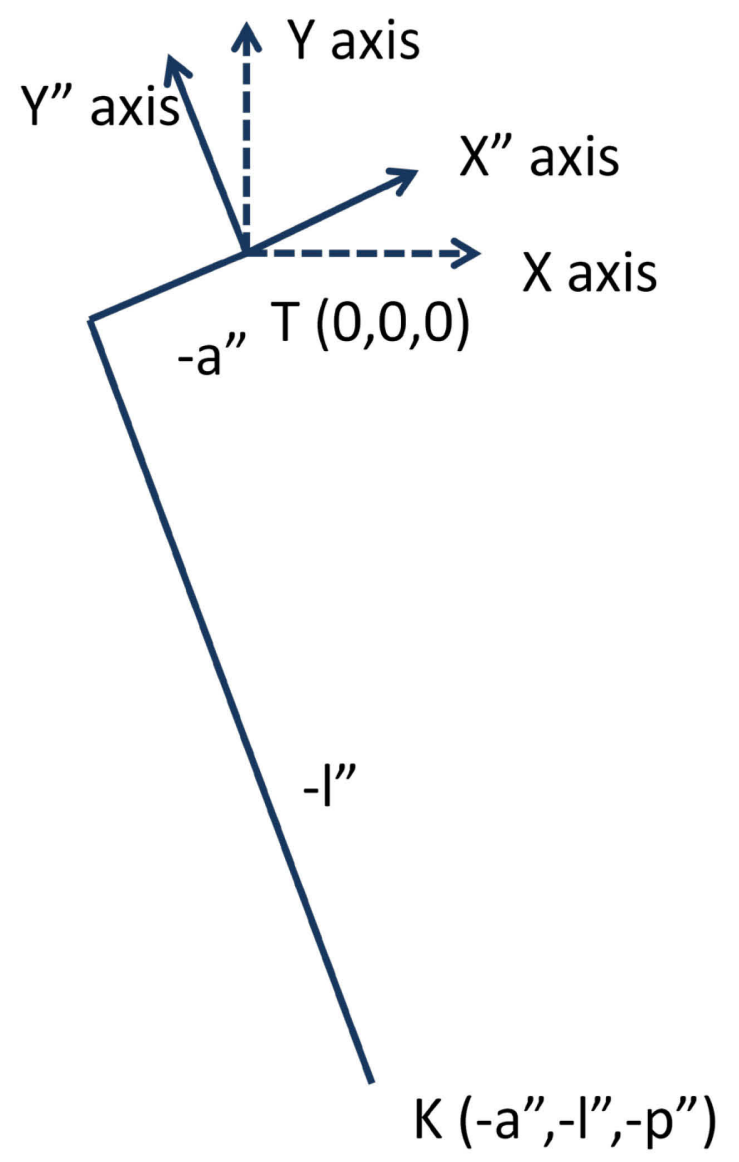

Figure 65: Point $\mathrm{K}$ is offset from point $\mathrm{T}$ by fixed distance 


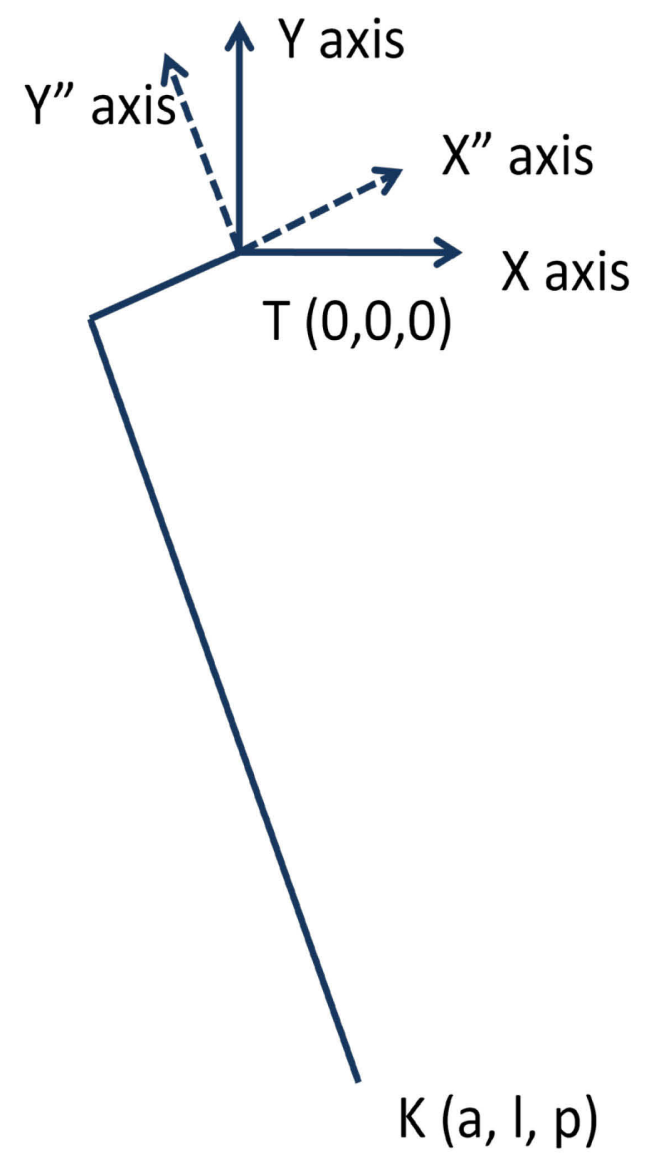

Figure 66: Coordinates of point $K$ with respect to point $T$ in camera frame 


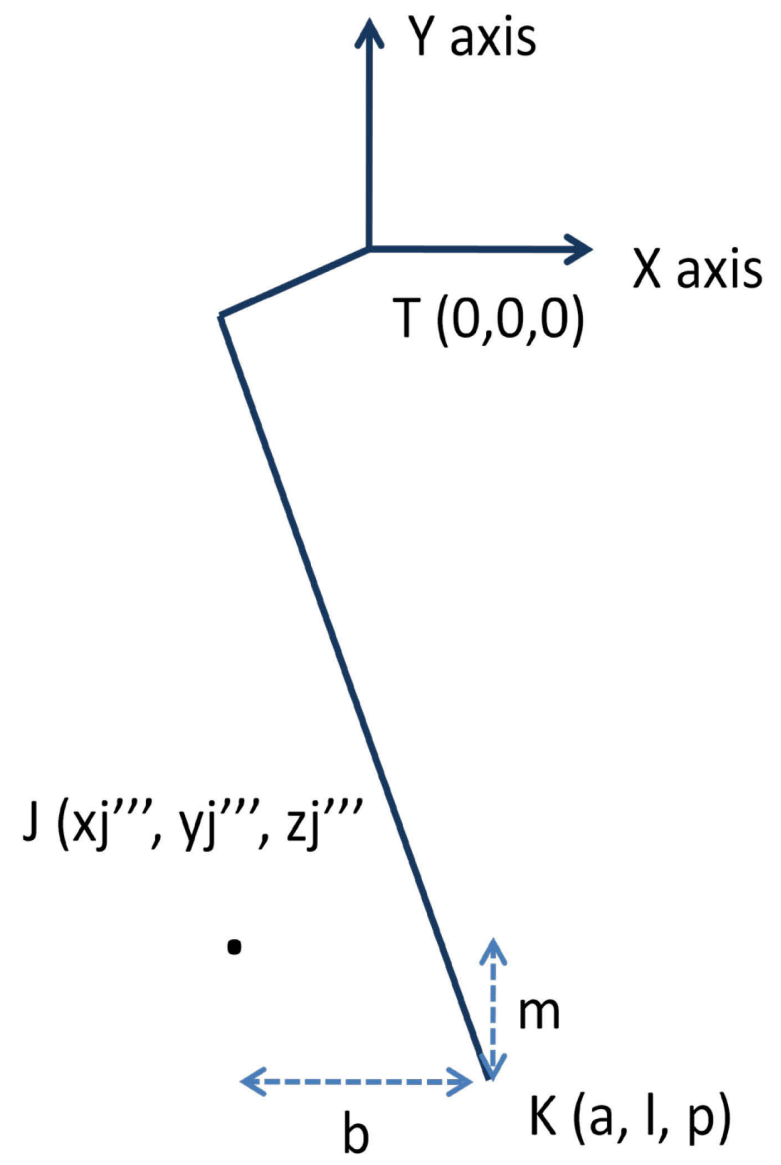

Figure 67: Coordinates of $\mathbf{J}$ using coordinates of $K$ and offset measurements 


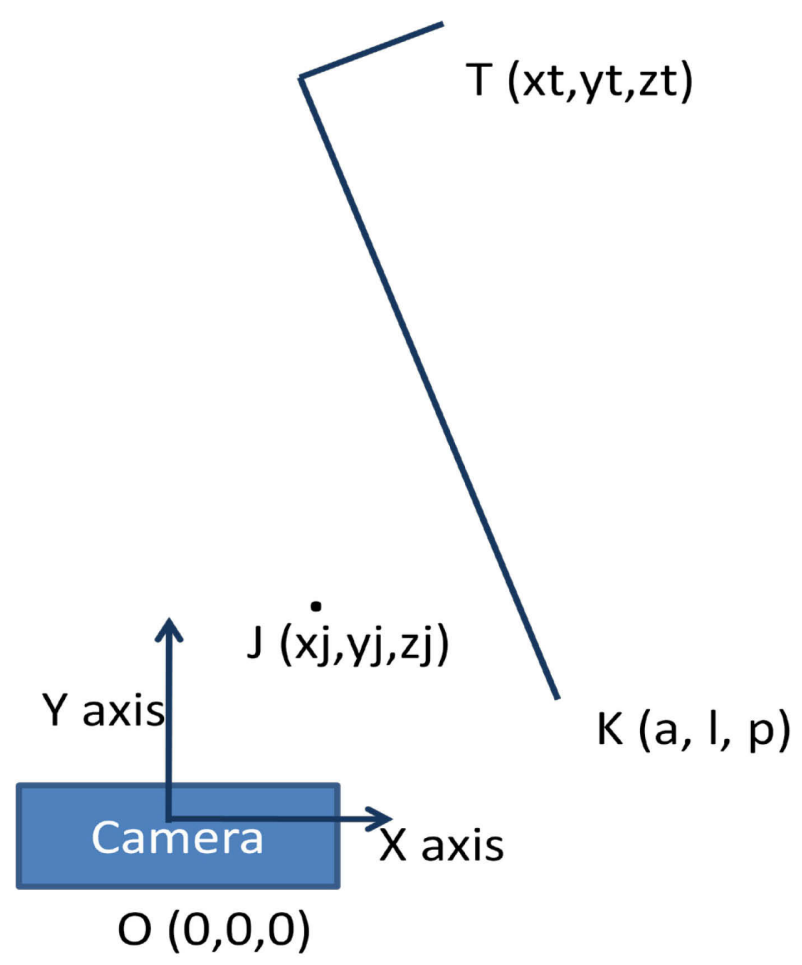

Figure 68: Global coordinates of $\mathbf{J}$ in the global camera system references from local target coordinates of talus target $T$ 
$\left[\begin{array}{l}x t \\ y t \\ z t\end{array}\right]$

Thus the actual coordinates of point $J$ with respect to camera origin $(x j, y j, z j)$ are given by:

$$
\left[\begin{array}{l}
x j \\
y j \\
z j
\end{array}\right]=\left[\begin{array}{l}
x^{\prime \prime \prime} \\
y j^{\prime \prime \prime} \\
z^{\prime \prime \prime}
\end{array}\right]+\left[\begin{array}{l}
x t \\
y t \\
z t
\end{array}\right]
$$

Thus,

$$
\left[\begin{array}{c}
x j \\
y j \\
z j
\end{array}\right]=\left[\begin{array}{l}
a \\
l \\
p
\end{array}\right]-\left[\begin{array}{c}
b \\
m \\
q
\end{array}\right]+\left[\begin{array}{c}
x t \\
y t \\
z t
\end{array}\right]
$$

\section{Step 5}

From data recorded and reported in the Labview data collection and target tracking vi. , we obtained the coordinates of both target arrays TT (xtt, ytt, ztt) and TN (xtn, ytn, ztn) which are attached to the talus and navicular bones respectively.

As seen in Figure 69, using this data from Labview and the coordinates of point $\mathrm{J}$, we get a vector (xjt, yjt, zjt), which defines the position of $J$ with respect to talus target and (xjn, yjn, zjn), which defines the position of $J$ with respect to navicular target. These vectors are obtained by subtraction of coordinates of point TT from coordinates of point $J$ and coordinates of point TN from coordinates of point $\mathrm{J}$.

$$
\begin{aligned}
& {\left[\begin{array}{l}
x j t \\
y j t \\
z j t
\end{array}\right]=\left[\begin{array}{l}
x j \\
y j \\
z j
\end{array}\right]-\left[\begin{array}{l}
x t t \\
y t t \\
z t t
\end{array}\right]} \\
& {\left[\begin{array}{l}
x j n \\
y j n \\
z j n
\end{array}\right]=\left[\begin{array}{l}
x j \\
y j \\
z j
\end{array}\right]-\left[\begin{array}{l}
x t n \\
y t n \\
z t n
\end{array}\right]}
\end{aligned}
$$

\section{Step 6}

As seen in Figure 70, joint location coordinates $(\mathrm{J})$ determined with respect to these vectors are converted into respective target frames of the talus and navicular target arrays using transformation matrix A. 


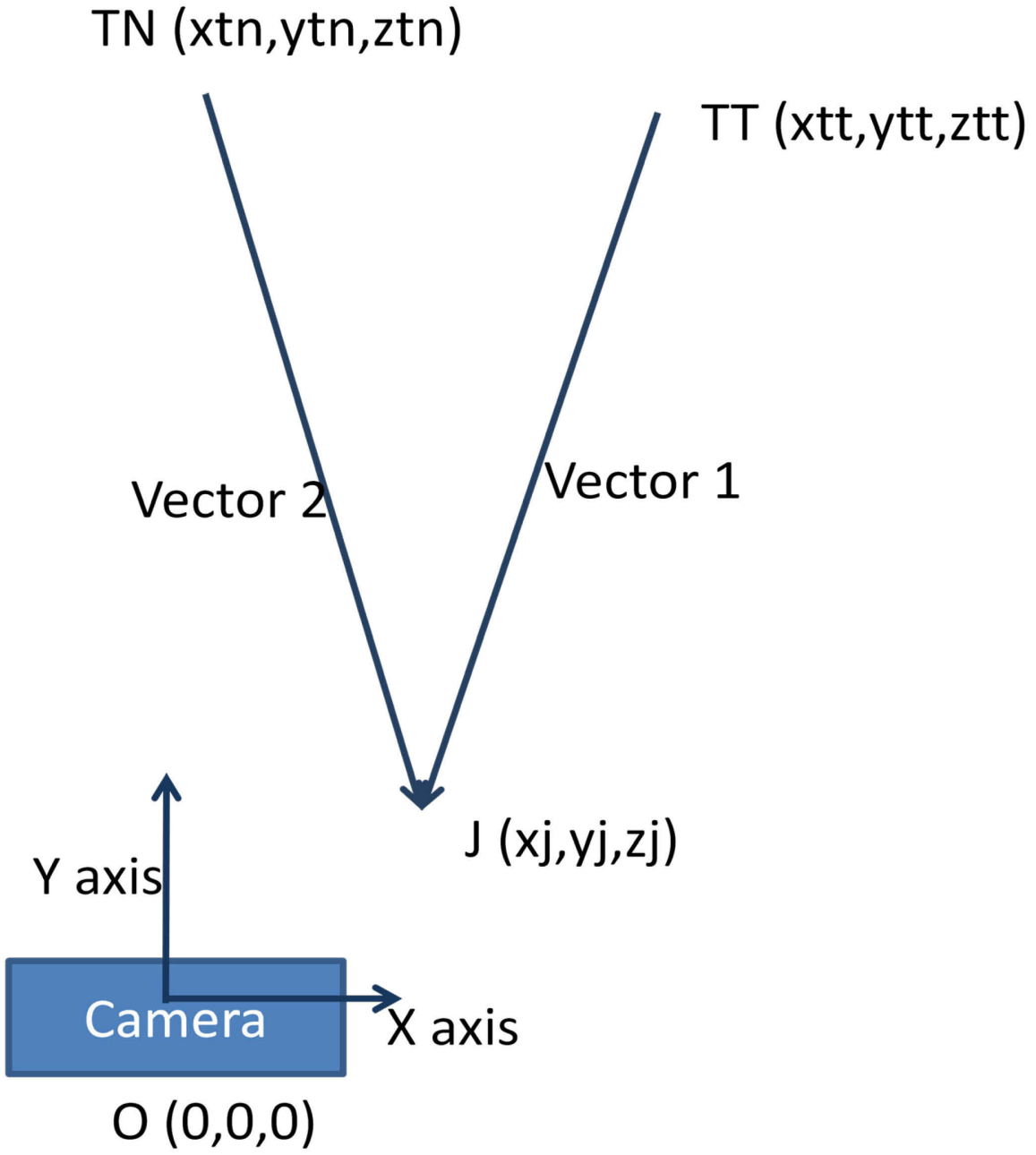

Figure 69: Vectors from each target to point $\mathbf{J}$ 


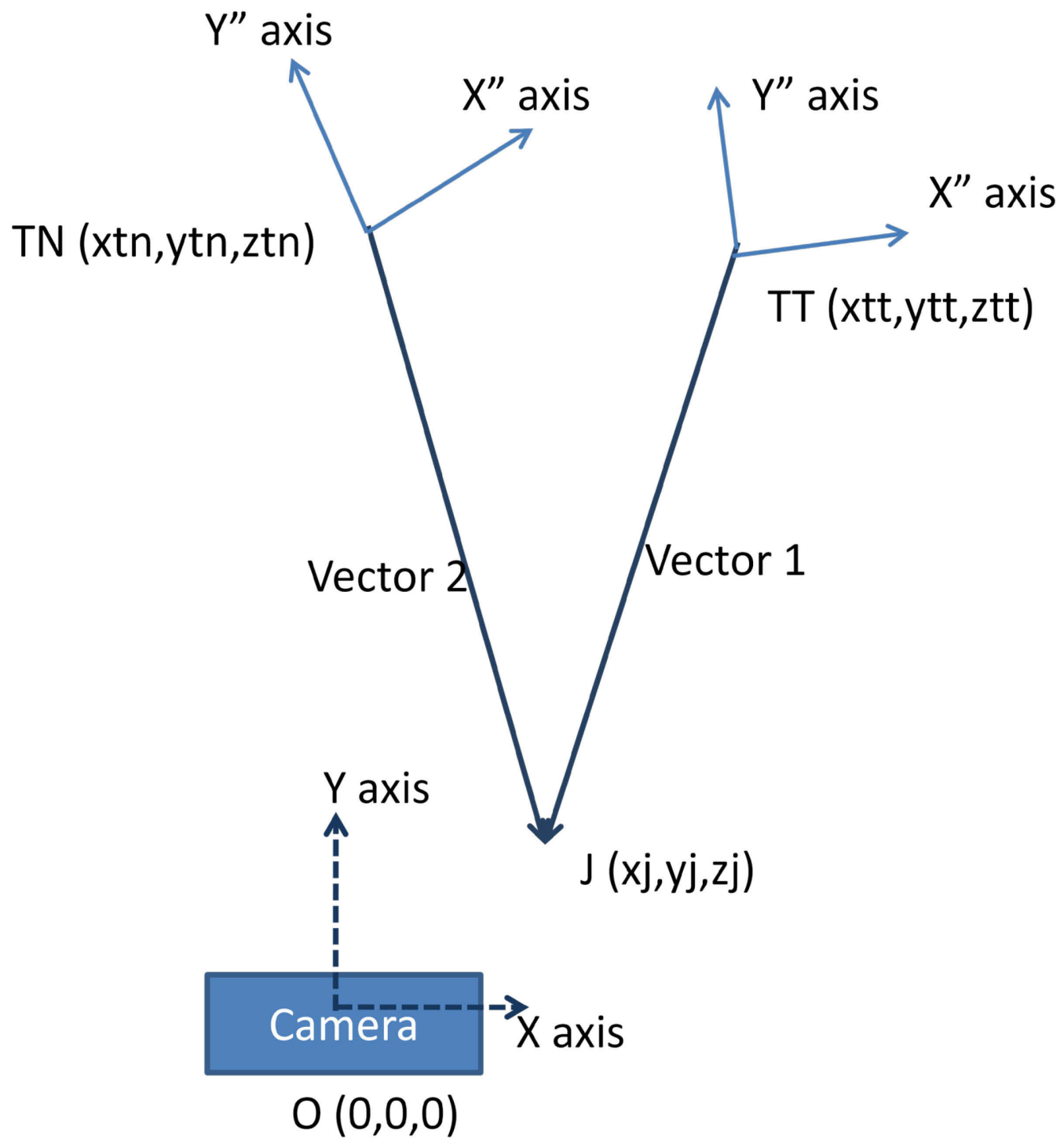

Figure 70: Conversion of vectors into individual target reference frames 


$$
\begin{gathered}
{\left[\begin{array}{l}
\text { xjt" } \\
\text { yjt" } \\
\text { zjt" }
\end{array}\right]=\left[\begin{array}{ccc}
\mathrm{C} 1 \mathrm{C} 2 & \mathrm{~S} 1 \mathrm{C} 2 & -\mathrm{S} 2 \\
-\mathrm{S} 1 \mathrm{C} 3+\mathrm{C} 1 \mathrm{~S} 2 \mathrm{~S} 3 & \mathrm{C} 1 \mathrm{C} 3+\mathrm{S} 1 \mathrm{~S} 2 \mathrm{~S} 3 & \mathrm{C} 2 \mathrm{~S} 3 \\
\mathrm{~S} 1 \mathrm{~S} 3+\mathrm{C} 1 \mathrm{~S} 2 \mathrm{C} 3 & -\mathrm{C} 1 \mathrm{~S} 3+\mathrm{S} 1 \mathrm{~S} 2 \mathrm{C} 3 & \mathrm{C} 2 \mathrm{C} 3
\end{array}\right]\left[\begin{array}{c}
\mathrm{xjt} \\
\mathrm{yjt} \\
\mathrm{zjt}
\end{array}\right]} \\
{\left[\begin{array}{l}
\mathrm{xjn} " \\
\text { yjn"} \\
\text { zjn" }
\end{array}\right]=\left[\begin{array}{ccc}
\mathrm{C} 1 \mathrm{C} 2 & \mathrm{~S} 1 \mathrm{C} 2 & -\mathrm{S} 2 \\
-\mathrm{S} 1 \mathrm{C} 3+\mathrm{C} 1 \mathrm{~S} 2 \mathrm{~S} 3 & \mathrm{C} 1 \mathrm{C} 3+\mathrm{S} 1 \mathrm{~S} 2 \mathrm{~S} 3 & \mathrm{C} 2 \mathrm{~S} 3 \\
\mathrm{~S} 1 \mathrm{~S} 3+\mathrm{C} 1 \mathrm{~S} 2 \mathrm{C} 3 & -\mathrm{C} 1 \mathrm{~S} 3+\mathrm{S} 1 \mathrm{~S} 2 \mathrm{C} 3 & \mathrm{C} 2 \mathrm{C} 3
\end{array}\right]\left[\begin{array}{c}
\mathrm{xjn} \\
\mathrm{yjn} \\
\mathrm{zjn}
\end{array}\right]}
\end{gathered}
$$

\section{Step 7}

These vectors $\left[\begin{array}{l}x j t " \\ y j t " \\ z j t "\end{array}\right]$ and $\left[\begin{array}{l}x j n " \\ y j n " \\ z j n "\end{array}\right]$ are the vectors corresponding to the location of J with respect to talus and navicular targets in the target reference frames at time zero.

\section{Steps 8 and 9}

As seen in Figure 71, at each given instance in time, using the instantaneous coordinate and rotation information of talus and navicular targets, we can find the coordinates of point $\mathrm{J}$ using following rotation and addition matrices:

$$
\begin{aligned}
& {\left[\begin{array}{c}
\mathrm{xjt} \\
\mathrm{yjt} \\
\mathrm{zjt}
\end{array}\right]=\left[\begin{array}{ccc}
\mathrm{C} 1 \mathrm{C} 2 & \mathrm{C} 1 \mathrm{~S} 2 \mathrm{~S} 3-\mathrm{S} 1 \mathrm{C} 3 & \mathrm{C} 1 \mathrm{~S} 2 \mathrm{C} 3+\mathrm{S} 1 \mathrm{~S} 3 \\
\mathrm{~S} 1 \mathrm{C} 2 & \mathrm{~S} 1 \mathrm{~S} 2 \mathrm{~S} 2+\mathrm{C} 1 \mathrm{C} 3 & \mathrm{~S} 1 \mathrm{~S} 2 \mathrm{C} 3-\mathrm{C} 1 \mathrm{~S} 3 \\
-\mathrm{S} 2 & \mathrm{C} 2 \mathrm{~S} 3 & \mathrm{C} 2 \mathrm{C} 3
\end{array}\right]\left[\begin{array}{c}
\mathrm{xjt} " \\
\mathrm{yjt} \\
\mathrm{zjt}
\end{array}\right]+\left[\begin{array}{c}
\mathrm{xtt} \\
\mathrm{ytt} \\
\mathrm{ztt}
\end{array}\right]} \\
& {\left[\begin{array}{c}
\text { xjn } \\
\text { yjn } \\
\text { zjn }
\end{array}\right]=\left[\begin{array}{ccc}
\text { C1C2 } & \text { C1S2S3 }- \text { S1C3 } & \text { C1S2C3 + S1S3 } \\
\text { S1C2 } & \text { S1S2S2 + C1C3 } & \text { S1S2C3 - C1S3 } \\
-S 2 & \text { C2S3 } & \text { C2C3 }
\end{array}\right]\left[\begin{array}{c}
\text { xjn" } \\
y j n " \\
z j n "
\end{array}\right]+\left[\begin{array}{c}
\text { xtn } \\
y t n \\
z \text { ztn }
\end{array}\right]}
\end{aligned}
$$

\section{Step 10}

If there is no relative translation between the two bones, the point $\mathrm{J}$ as defined with respect to each target will be the and hence,

$$
\mathrm{JN}-\mathrm{JT}=0
$$

i.e.,

$$
\left[\begin{array}{l}
\mathrm{xjn} \\
\mathrm{yjn} \\
\mathrm{zjn}
\end{array}\right]-\left[\begin{array}{l}
\mathrm{xjt} \\
\mathrm{yjt} \\
\mathrm{zjt}
\end{array}\right]=0
$$




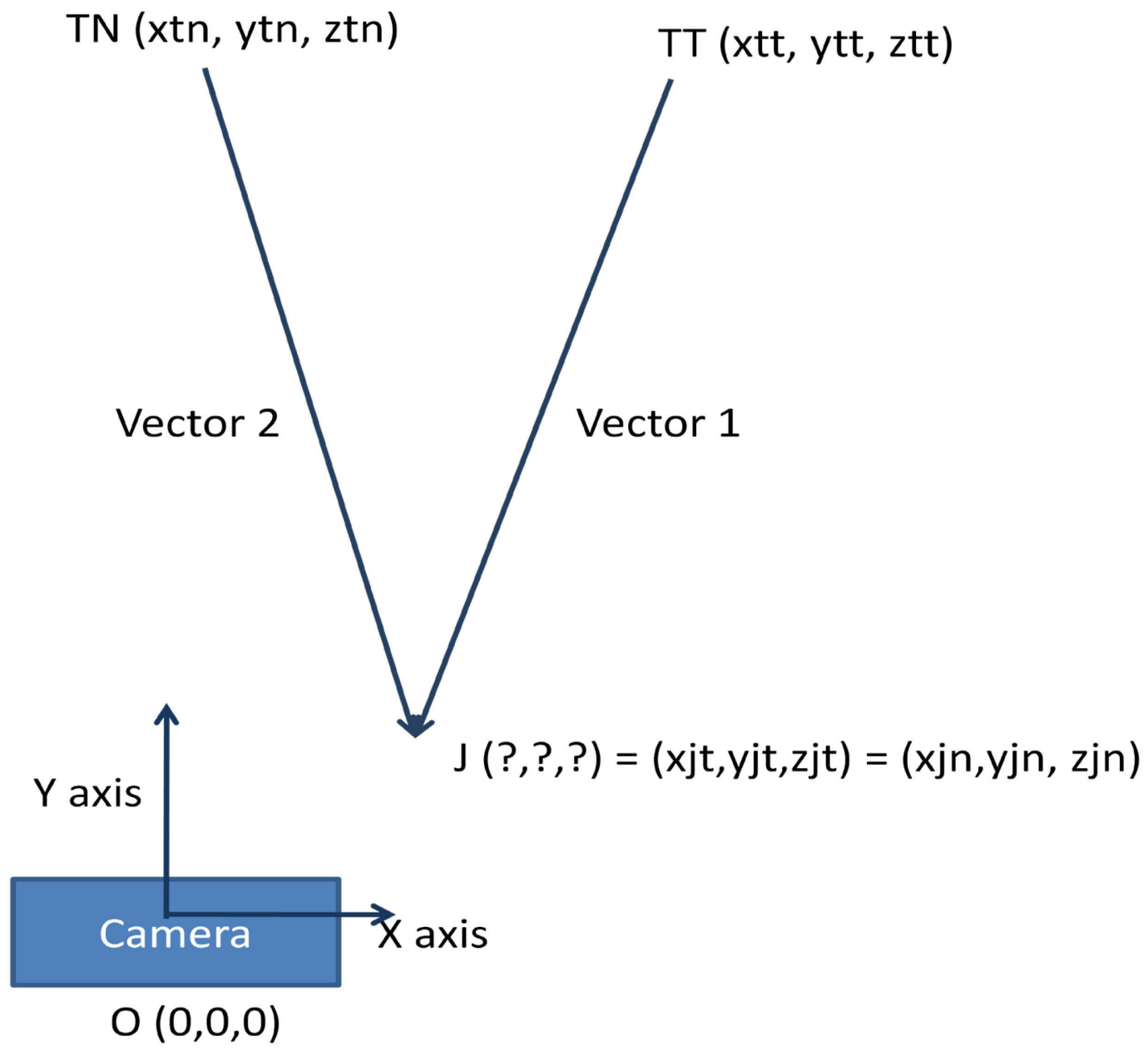

Figure 71: Transformation of target coordinates into coordinates of $\mathbf{J}$ using appropriate vector 
With relative translation between the bones, the target arrays move with respect to each other and hence point $J$ as defines relative to each target array would be different. The difference between the two results for point $\mathrm{J}$ is the relative translations along each axis of the camera frame. We will call these translations as transx, transy and transz along the $\mathrm{X}, \mathrm{Y}$ and $\mathrm{Z}$ axes of the camera reference frame.

$$
\left[\begin{array}{c}
\operatorname{transx} \\
\operatorname{transy} \\
\operatorname{transz}
\end{array}\right]=\left[\begin{array}{c}
x j n \\
y j n \\
z j n
\end{array}\right]-\left[\begin{array}{l}
x j t \\
y j t \\
z j t
\end{array}\right]
$$

\section{Step 11}

The next step was to determine the relative translations in the joint frame, which was a coordinate frame, aligned with the natural orientation of the native joint plane. It has been described earlier and depicted in the Figures 72 and 73.

As previously described, the joint frame is rotated by $\alpha 1$ (Figure 72) about $\mathrm{Z}$ axis, $\alpha 2$ about $\mathrm{Y}$ axis (Figure 73) and $\alpha 3$ about $\mathrm{X}$ axis. Of these, $\alpha 3$ was assumed to be zero, since there was no proper radiographic view to determine its magnitude.

To transform the data into joint frame, we used the transformation matrix $\mathrm{C}$ which has been previously derived.

$$
\begin{aligned}
& {\left[\begin{array}{l}
\mathrm{xjt}^{\prime} \\
\mathrm{yjt}^{\prime} \\
\mathrm{zjt}^{\prime}
\end{array}\right]=\left[\begin{array}{ccc}
\mathrm{C} 1 \mathrm{C} 2 & \mathrm{~S} 1 & -\mathrm{C} 1 \mathrm{~S} 2 \\
-\mathrm{S} 1 \mathrm{C} 2 \mathrm{C} 3+\mathrm{S} 2 \mathrm{~S} 3 & \mathrm{C} 1 \mathrm{C} 3 & \mathrm{~S} 1 \mathrm{~S} 2 \mathrm{C} 3+\mathrm{C} 2 \mathrm{~S} 3 \\
\mathrm{~S} 1 \mathrm{C} 2 \mathrm{~S} 3+\mathrm{S} 2 \mathrm{C} 3 & -\mathrm{C} 2 \mathrm{~S} 3 & -\mathrm{S} 1 \mathrm{~S} 2 \mathrm{~S} 3+\mathrm{C} 2 \mathrm{C} 3
\end{array}\right]\left[\begin{array}{c}
\text { xjt } \\
y j t \\
z j t
\end{array}\right]} \\
& {\left[\begin{array}{c}
\text { xjn' } \\
y_{j n}^{\prime} \\
\text { zjn' }
\end{array}\right]=\left[\begin{array}{ccc}
\mathrm{C} 1 \mathrm{C} 2 & \mathrm{~S} 1 & -\mathrm{C} 1 \mathrm{~S} 2 \\
-\mathrm{S} 1 \mathrm{C} 2 \mathrm{C} 3+\mathrm{S} 2 \mathrm{~S} 3 & \mathrm{C} 1 \mathrm{C} 3 & \mathrm{~S} 1 \mathrm{~S} 2 \mathrm{C} 3+\mathrm{C} 2 \mathrm{~S} 3 \\
\mathrm{~S} 1 \mathrm{C} 2 \mathrm{~S} 3+\mathrm{S} 2 \mathrm{C} 3 & -\mathrm{C} 2 \mathrm{~S} 3 & -\mathrm{S} 1 \mathrm{~S} 2 \mathrm{~S} 3+\mathrm{C} 2 \mathrm{C} 3
\end{array}\right]\left[\begin{array}{c}
\text { xjn } \\
y j n \\
z j n
\end{array}\right]}
\end{aligned}
$$

where,

$$
\begin{aligned}
& \mathrm{C} 1=\cos \alpha_{1} \\
& \mathrm{C} 2=\cos \alpha_{2} \\
& \mathrm{C} 3=\cos \alpha_{3} \\
& \mathrm{~S} 1=\sin \alpha_{1} \\
& \mathrm{~S} 2=\sin \alpha_{2}
\end{aligned}
$$




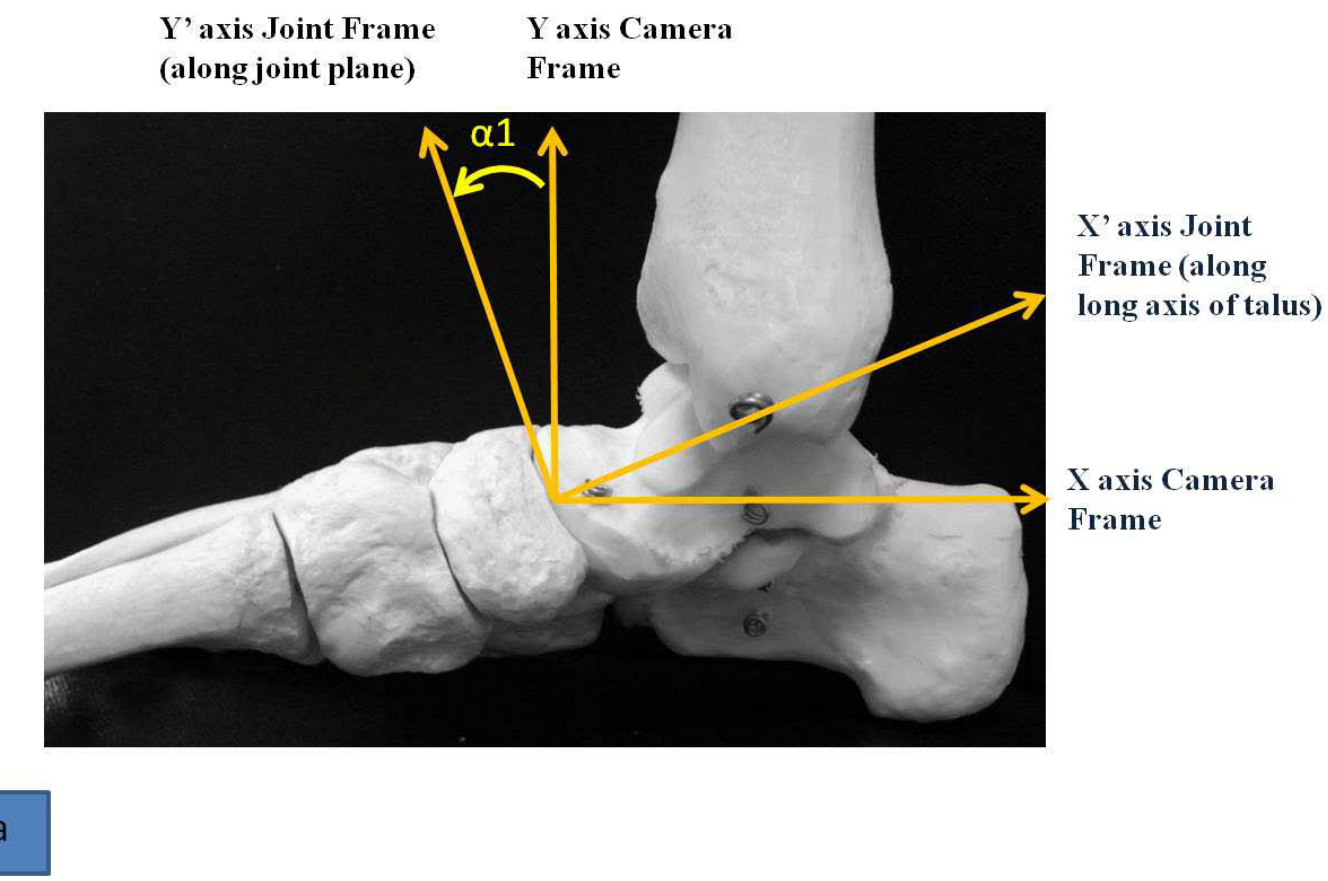

Figure 72: Joint frame orientation (medial view) 


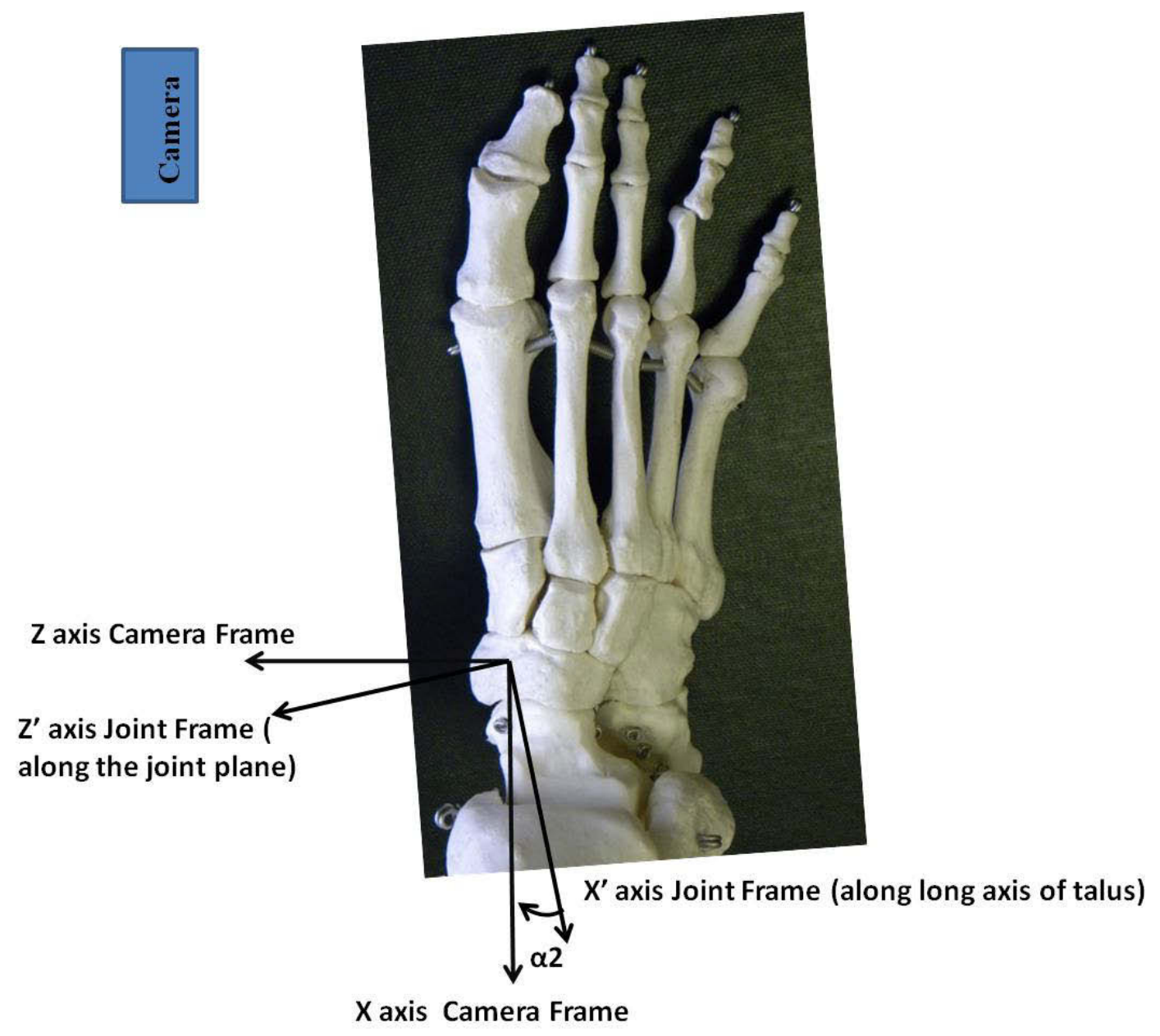

Figure 73: Joint frame orientation (top view) 
and,

$$
\mathrm{S} 3=\sin \alpha_{3}
$$

$\alpha_{1} \quad=\quad$ angle of rotation of joint frame with respect to camera frame about $\mathrm{Z}$ axis

$\alpha_{2}=$ angle of rotation of joint frame with respect to camera frame about $\mathrm{Y}$ axis

$\alpha_{3}=$ angle of rotation of joint frame with respect to camera frame about $\mathrm{X}$ axis

Thus we transform the joint coordinates JT and JN obtained from using talus and navicular data into the joint frame.

\section{Step 12}

Once again, taking the difference JN-JT will give us transx', transy' and transz', which are the separations along the $\mathrm{X}^{\prime}, \mathrm{Y}^{\prime}$ and $\mathrm{Z}^{\prime}$ axes aligned with the joint frame.

$$
\left[\begin{array}{l}
\operatorname{transx}^{\prime} \\
\text { transy' }^{\prime} \\
\text { transz' }
\end{array}\right]=\left[\begin{array}{l}
\mathrm{xjn}^{\prime} \\
\mathrm{yjn}^{\prime} \\
\mathrm{zjn}^{\prime}
\end{array}\right]-\left[\begin{array}{l}
\mathrm{xjt}^{\prime} \\
\mathrm{yjt}^{\prime} \\
\mathrm{zjt}^{\prime}
\end{array}\right]
$$

This process (Step 8 to Step 12) was repeated for each time-point to determine the relative separations between the bones in the camera reference frame as well as the joint reference frame.

\section{DATA PROCESSING (ROTATION)}

The Labview Vi reports the relative rotations between the two targets after accounting for the distance of the targets from the camera origin. Thus the relative rotations would be the same as the relative rotation between the two bones in the camera frame. Thus we have the rotations about $\mathrm{X}, \mathrm{Y}$ and $\mathrm{Z}$ axes as Rx, Ry and Rz respectively.

To get the relative rotations about $\mathrm{X}^{\prime}, \mathrm{Y}^{\prime}$ and $\mathrm{Z}^{\prime}$ axes i.e., corresponding axes of the joint frame, we use the transformation matrix A.

$$
\left[\begin{array}{l}
\mathrm{Rx}^{\prime} \\
\mathrm{Ry}^{\prime} \\
\mathrm{Rz}^{\prime}
\end{array}\right]=\left[\begin{array}{ccc}
\mathrm{C} 1 \mathrm{C} 2 & \mathrm{~S} 1 \mathrm{C} 2 & -\mathrm{S} 2 \\
-\mathrm{S} 1 \mathrm{C} 3+\mathrm{C} 1 \mathrm{~S} 2 \mathrm{~S} 3 & \mathrm{C} 1 \mathrm{C} 3+\mathrm{S} 1 \mathrm{~S} 2 \mathrm{~S} 3 & \mathrm{C} 2 \mathrm{~S} 3 \\
\mathrm{~S} 1 \mathrm{~S} 3+\mathrm{C} 1 \mathrm{~S} 2 \mathrm{C} 3 & -\mathrm{C} 1 \mathrm{~S} 3+\mathrm{S} 1 \mathrm{~S} 2 \mathrm{C} 3 & \mathrm{C} 2 \mathrm{C} 3
\end{array}\right]\left[\begin{array}{l}
\mathrm{Rx} \\
\mathrm{Ry} \\
\mathrm{Rz}
\end{array}\right]
$$

where,

$$
\begin{aligned}
& \mathrm{C} 1=\cos \alpha_{1} \\
& \mathrm{C} 2=\cos \alpha_{2} \\
& \mathrm{C} 3=\cos \alpha_{3}
\end{aligned}
$$




$$
\begin{aligned}
& \mathrm{S} 1=\sin \alpha_{1} \\
& \mathrm{~S} 2=\sin \alpha_{2} \\
& \mathrm{~S} 3=\sin \alpha_{3}
\end{aligned}
$$

and where the angle of rotation of the joint frame with respect to camera frame is $\alpha_{1}$ about $Z$ axis, $\alpha_{2}$ about $Y$ axis and $\alpha_{3}$ about $X$ axis. Thus the relative rotations between the talus and the navicular bone, $\mathrm{Rx}^{\prime}, \mathrm{Ry}^{\prime}$ and $\mathrm{Rz}$, about $\mathrm{X}^{\prime}, \mathrm{Y}^{\prime}$ and $\mathrm{Z}^{\prime}$ axes, respectively, of the joint frame are calculated. 


\section{APPENDIX F: VALIDATION OF DATA PROCESSING METHODOLOGY}

\section{OBJECTIVE}

To validate data processing equations using automated software.

\section{DESCRIPTION OF MODEL}

A model was constructed using a dynamic simulation software - Visual Nastran, which was used for validation of equations.

The coordinates of targets and the offsets of the targets from the user defined point $\mathbf{J}$ which lies in the joint space were known. The coordinates of the point $\mathbf{J}$ were to be determined using the talus and navicular target coordinates. Further, the relative separation in the camera frame and in the joint frame is to be determined.

The Visual Nastran model can be seen in Figure 74. All bodies were weightless. A sphere was created in the 3 dimensional workspace space at the talus coordinates at time zero as provided by the camera data file to represent the talus target. Three cylindrical rods were created to represent the offsets in the orientation of the target and the K-wire. Thus a rod was constructed from sphere extending in the negative $\mathrm{Z}$ direction for $35 \mathrm{~mm}$ ending in a sphere. A second rod was constructed of $7.6 \mathrm{~mm}$ extending from this second sphere in the negative $\mathrm{X}$ direction and ending in a third sphere. A third cylinder was constructed extending from this sphere extending in the negative $\mathrm{Y}$ direction of length matching the $\mathrm{K}$ wire of the specimen used for validation. This cylinder ended in a sphere which represents the lower end of the $\mathrm{K}$ wire. All this together constituted the talus target assembly. The entire structure was rotated by the angles as provided by the camera data file. The process was repeated using the specifications of the navicular target to create the navicular target assembly.

A sphere was constructed at coordinates determined by using the offset measurements from the lower end of the $\mathrm{K}$ wire as determined from radiographs. This sphere was called JCT. This sphere was linked by rigid constraints to the lower end of the talus target assembly and thus this sphere moved if the talus target was moved.

Another sphere was created at the same coordinates as JCT but this sphere was linked using rigid constraints to lower end of navicular target assembly. This sphere was called JCN.

The coordinates of JCT and JCN were recorded by inputting various coordinates for talus and navicular targets for all time points as provided by the camera data file (Figure 75). This data was stored and compared with the coordinates of JCT and JCN obtained using the transformation matrices and data processing equations to prove the validation of the data processing methodology. 


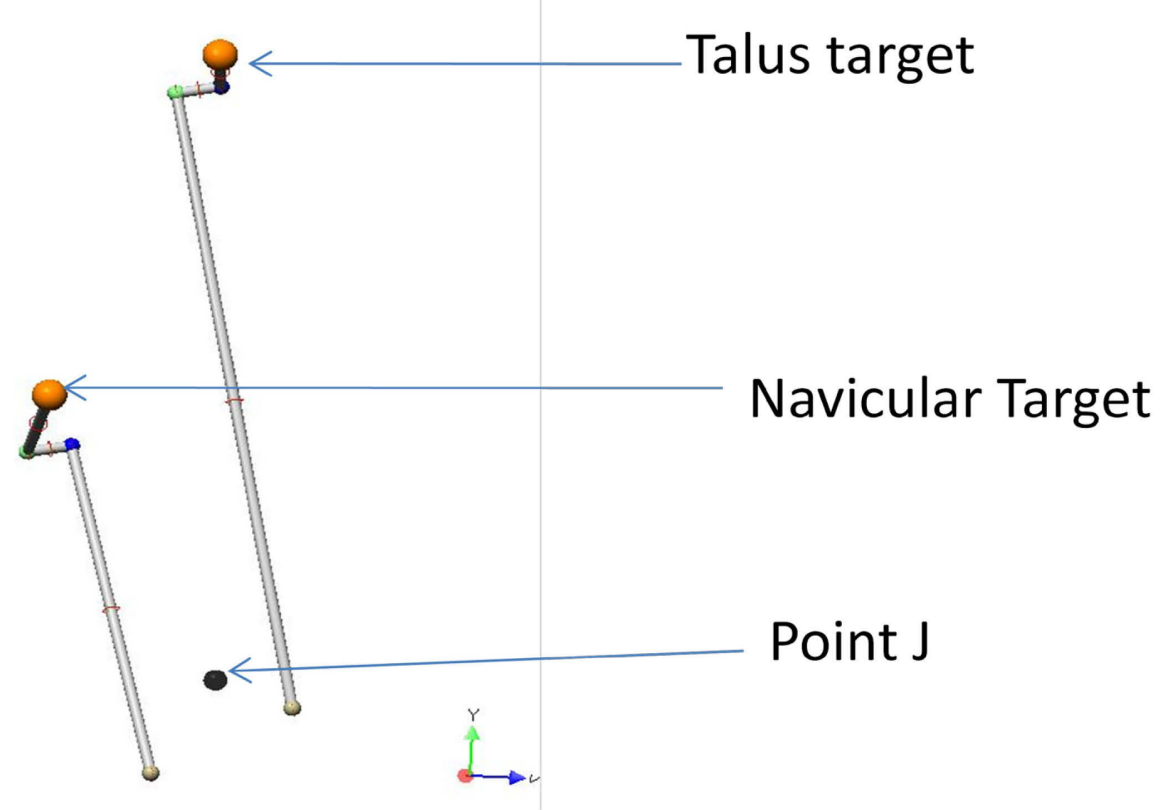

Figure 74: Visual Nastran model showing two targets and point $J$ at time zero 


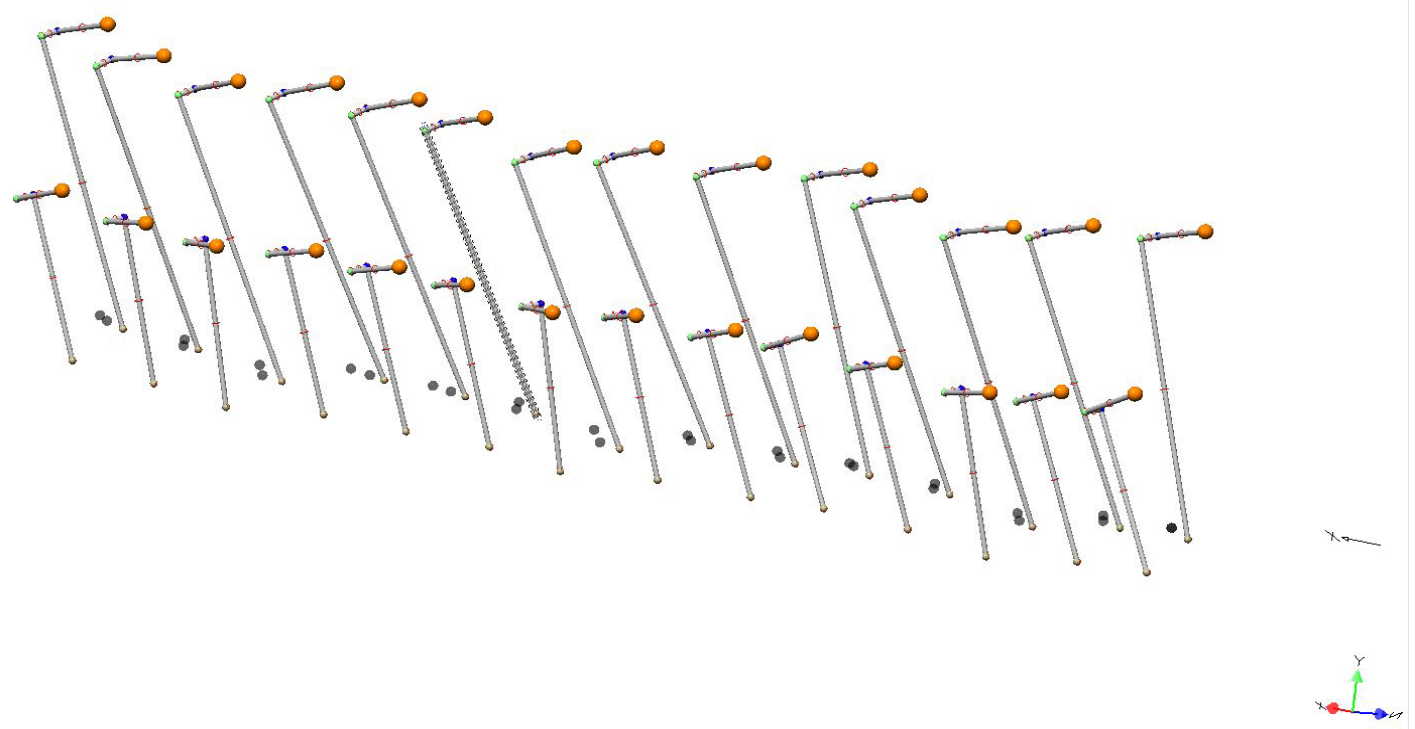

Figure 75: Visual Nastran model showing the two targets and point $\mathbf{J}$ at various time points 
To prove the transformation between the camera frame and the joint frame, another model was created using Visual Nastran (Figure 76). In this, a sphere was created at the origin and three mutually perpendicular cylinders were created such that the sphere was at the midpoint of each cylinder. These three cylinders represented the three axes X,Y,Z. The sphere was rotated by 10 degrees about $\mathrm{Y}$ axis, followed by 30 degrees about $\mathrm{X}$ axis. The axes became $\mathrm{X}^{\prime}, \mathrm{Y}^{\prime}, \mathrm{Z}^{\prime}$.

An actuator was created connecting one end of the $X^{\prime}$ rod to the center of the sphere. This was called $X^{\prime}$ actuator. The process was repeated to create similar $Y^{\prime}$ and $Z^{\prime}$ actuators.

The $X^{\prime}$ actuator was programmed to move $20 \mathrm{~mm}$ along the $X^{\prime}$ axis. Thus the new coordinates of the center of sphere in $X^{\prime} Y^{\prime} Z^{\prime}$ frame were $(20,0,0)$. The coordinates of center of sphere in the XYZ frame were reported by Visual Nastran directly. These coordinates were processed using the camera to joint frame transformation matrix using angles 30 and 10 about $Z$ and $Y$ axes respectively to find the coordinates of center of sphere in $X^{\prime} Y^{\prime} Z^{\prime}$ frame. These coordinates were compared with the expected coordinates $(20,0,0)$. The process was repeated using the $Y^{\prime}$ and $Z^{\prime}$ actuators to prove the validity of the transformation matrix. 

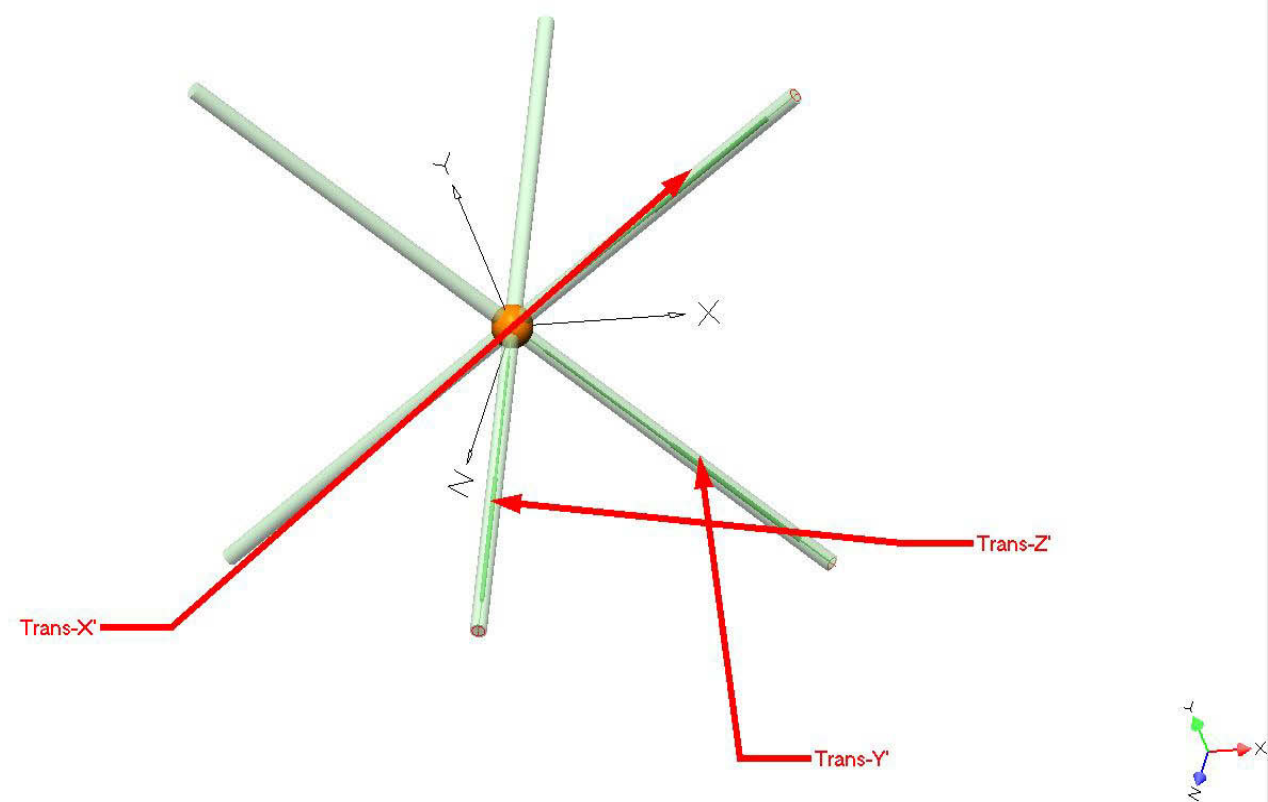

Figure 76: Visual Nastran model showing the validation of reference frame transformation 


\section{APPENDIX G: TABLES OF RESULTS}

The following tables contain the results of translations along $\mathrm{X}, \mathrm{Y}$ and $\mathrm{Z}$ axes of camera frame (Tables 1 to 6), rotations about $\mathrm{X}, \mathrm{Y}$ and $\mathrm{Z}$ axes of camera frame (Tables 7 to 12), translations along $X^{\prime}, Y^{\prime}$ and $Z^{\prime}$ axes of joint frame (Tables 13 to 18) and rotations about $\mathrm{X}^{\prime}, \mathrm{Y}^{\prime}$ and $\mathrm{Z}^{\prime}$ axes of joint frame (Tables 19 to 24).

Table 1: Relative translation along $X$ axis (harvested/native state) in camera frame

\begin{tabular}{lcccc}
\hline Harvested & $\begin{array}{c}\text { Internal } \\
\text { Rotation } \\
\text { Sequential } \\
\text { Test }\end{array}$ & $\begin{array}{c}\text { Internal } \\
\text { Rotation } \\
\text { Simultaneous }\end{array}$ & $\begin{array}{c}\text { External } \\
\text { Rotation } \\
\text { Sequential } \\
\text { Test }\end{array}$ & $\begin{array}{c}\text { External } \\
\text { Rotation } \\
\text { Simultaneous }\end{array}$ \\
\hline Spec\#1 R & 1.1 & 0.5 & 1.5 & Test \\
Spec\#2 L & 0.3 & 0.6 & 1.5 & 2.2 \\
Spec\#4 R & 0.4 & 2.6 & 2.4 & 0.2 \\
Spec\#14 R & 0.0 & 2.2 & 1.7 & 1.4 \\
Spec\#17 R & 2.8 & 0.2 & 0.3 & 4.5 \\
Spec\# 20 R & 2.5 & 0.8 & 1.6 & 0.7 \\
Sepc\#21 R & 1.1 & 0.1 & 0.2 & 0.7 \\
Mean & $\mathbf{1 . 2}$ & $\mathbf{1 . 0}$ & $\mathbf{1 . 3}$ & 0.2 \\
Std & $\mathbf{1 . 1}$ & $\mathbf{1 . 0}$ & $\mathbf{0 . 8}$ & $\mathbf{1 . 4}$ \\
& & & & $\mathbf{1 . 5}$ \\
\hline & Internal & Internal & External & External \\
Harvested & Rotation & Rotation & Rotation & Rotation \\
(Plate + Screw) & Sequential & Simultaneous & Sequential & Simultaneous \\
& Test & Test & Test & Test \\
\hline Spec\#3 L & 0.3 & 1.7 & 1.2 & 1.5 \\
Spec\#5 L & 2.5 & 1.2 & 3.7 & 4.0 \\
Spec\#6 L & 3.3 & 1.6 & 2.1 & 1.4 \\
Spec\#9 L & 6.4 & 6.0 & 2.2 & 6.0 \\
Spec\#13 R & 0.7 & 1.2 & 0.8 & 0.3 \\
Spec\#15 L & 1.2 & 1.1 & 0.3 & 0.7 \\
Mean & $\mathbf{2 . 4}$ & $\mathbf{2 . 1}$ & $\mathbf{1 . 7}$ & $\mathbf{2 . 3}$ \\
Std & $\mathbf{2 . 3}$ & $\mathbf{1 . 9}$ & $\mathbf{1 . 2}$ & $\mathbf{2 . 2}$ \\
& & & & \\
\hline
\end{tabular}


Table 2: Relative translation along $X$ axis (instrumented state) in camera frame

\begin{tabular}{lcccc}
\hline \multicolumn{1}{c}{ Screws } & $\begin{array}{c}\text { Internal } \\
\text { Rotation } \\
\text { Sequential Test }\end{array}$ & $\begin{array}{c}\text { Internal } \\
\text { Rotation } \\
\text { Simultaneous } \\
\text { Test }\end{array}$ & $\begin{array}{c}\text { External } \\
\text { Rotation } \\
\text { Sequential Test }\end{array}$ & $\begin{array}{c}\text { External } \\
\text { Rotation } \\
\text { Simultaneous } \\
\text { Test }\end{array}$ \\
\hline Spec\#1 R & 2.3 & 0.7 & 1.8 & 2.9 \\
Spec\#2 L & 1.3 & 0.4 & 0.7 & 0.2 \\
Spec\#4 R & 0.7 & 0.4 & 2.9 & 0.8 \\
Spec\#14 R & 2.0 & 1.7 & 0.2 & 0.2 \\
Spec\#17 R & 1.9 & 1.5 & 1.9 & 0.6 \\
Spec\# 20 R & 1.2 & 0.4 & 2.1 & 3.4 \\
Sepc\#21 R & 1.0 & 0.2 & 1.6 & 1.4 \\
Mean & $\mathbf{1 . 5}$ & $\mathbf{0 . 8}$ & $\mathbf{1 . 6}$ & $\mathbf{1 . 4}$ \\
Std & $\mathbf{0 . 6}$ & $\mathbf{0 . 6}$ & $\mathbf{0 . 9}$ & $\mathbf{1 . 3}$ \\
& & & & External \\
& Internal & Internal & External & Rotation \\
Plate + Screw & Rotation & Rotation & Rotation & Simultaneous \\
& Sequential Test & Simultaneous & Sequential Test & Test \\
\hline Spec\#3 L & 4.2 & 0.2 & 1.9 & 1.0 \\
Spec\#5 L & 0.2 & 2.0 & 0.6 & 0.4 \\
Spec\#6 L & 0.6 & 2.1 & 1.5 & 2.2 \\
Spec\#9 L & 1.2 & 1.6 & 0.6 & 1.2 \\
Spec\#13 R & 0.5 & 0.2 & 0.4 & 0.8 \\
Spec\#15 L & 0.1 & 0.0 & $\mathbf{1 . 3}$ & $\mathbf{1 . 3}$ \\
Mean & $\mathbf{1 . 1}$ & $\mathbf{1 . 0}$ & $\mathbf{0 . 9}$ & $\mathbf{0 . 8}$ \\
Std & $\mathbf{1 . 6}$ & & & \\
\hline
\end{tabular}


Table 3: Relative translation along $Y$ axis (harvested/native state) in camera frame

\begin{tabular}{lcccc}
\hline $\begin{array}{c}\text { Harvested } \\
\text { (Screws) }\end{array}$ & $\begin{array}{c}\text { Internal } \\
\text { Rotation } \\
\text { Sequential Test }\end{array}$ & $\begin{array}{c}\text { Internal } \\
\text { Rotation } \\
\text { Simultaneous } \\
\text { Test }\end{array}$ & $\begin{array}{c}\text { External } \\
\text { Rotation } \\
\text { Sequential Test }\end{array}$ & $\begin{array}{c}\text { External } \\
\text { Rotation } \\
\text { Simultaneous } \\
\text { Test }\end{array}$ \\
\hline Spec\#1 R & 1.7 & 2.2 & 1.0 & 1.6 \\
Spec\#2 L & 0.1 & 0.3 & 0.3 & 0.6 \\
Spec\#4 R & 0.8 & 0.5 & 2.7 & 2.1 \\
Spec\#14 R & 0.8 & 0.7 & 1.2 & 2.6 \\
Spec\#17 R & 0.5 & 1.7 & 1.6 & 2.3 \\
Spec\# 20 R & 0.7 & 2.1 & 1.5 & 0.3 \\
Sepc\#21 R & 0.3 & 0.1 & 0.8 & 0.6 \\
Mean & $\mathbf{0 . 7}$ & $\mathbf{1 . 1}$ & $\mathbf{1 . 3}$ & $\mathbf{1 . 4}$ \\
Std & $\mathbf{0 . 5}$ & $\mathbf{0 . 9}$ & $\mathbf{0 . 8}$ & $\mathbf{0 . 9}$ \\
& & & & External \\
Harvested & Internal & Internal & External & Rotation \\
(Plate+ Screw) & Rotation & Rotation & Rotation & Simultaneous \\
& Simultaneous & Sequential Test & Test \\
\hline Spec\#3 L & 0.7 & 1.5 & 0.7 & 3.2 \\
Spec\#5 L & 1.7 & 0.8 & 1.7 & 1.3 \\
Spec\#6 L & 3.1 & 1.8 & 0.1 & 0.2 \\
Spec\#9 L & 0.4 & 1.0 & 0.3 & 0.8 \\
Spec\#13 R & 0.8 & 0.9 & 1.2 & 0.4 \\
Spec\#15 L & 0.7 & 2.9 & 0.7 & 0.4 \\
Mean & $\mathbf{1 . 2}$ & $\mathbf{1 . 5}$ & $\mathbf{0 . 8}$ & $\mathbf{1 . 1}$ \\
Std & $\mathbf{1 . 0}$ & $\mathbf{0 . 8}$ & $\mathbf{0 . 6}$ & \\
\hline
\end{tabular}


Table 4: Relative translation along $Y$ axis (instrumented state) in camera frame

\begin{tabular}{|c|c|c|c|c|}
\hline Screws & $\begin{array}{c}\text { Internal } \\
\text { Rotation } \\
\text { Sequential Test }\end{array}$ & $\begin{array}{c}\text { Internal } \\
\text { Rotation } \\
\text { Simultaneous } \\
\text { Test } \\
\end{array}$ & $\begin{array}{c}\text { External } \\
\text { Rotation } \\
\text { Sequential Test }\end{array}$ & $\begin{array}{c}\text { External } \\
\text { Rotation } \\
\text { Simultaneous } \\
\text { Test } \\
\end{array}$ \\
\hline Spec\#1 R & 2.9 & 2.7 & 0.6 & 0.8 \\
\hline Spec\#2 L & 2.4 & 1.5 & 3.8 & 4.1 \\
\hline Spec\#4 R & 0.6 & 0.3 & 0.8 & 1.4 \\
\hline Spec\#14 R & 0.3 & 0.7 & 0.5 & 0.2 \\
\hline Spec\#17 R & 1.7 & 2.1 & 0.4 & 2.9 \\
\hline Spec\# 20 R & 1.3 & 0.2 & 0.2 & 0.1 \\
\hline Sepc\#21 R & 0.1 & 0.9 & 0.2 & 0.1 \\
\hline Mean & 1.3 & 1.2 & 0.9 & 1.4 \\
\hline Std & 1.1 & 0.9 & 1.3 & 1.6 \\
\hline Plate + Screw & $\begin{array}{c}\text { Internal } \\
\text { Rotation } \\
\text { Sequential Test }\end{array}$ & $\begin{array}{c}\text { Internal } \\
\text { Rotation } \\
\text { Simultaneous } \\
\text { Test } \\
\end{array}$ & $\begin{array}{c}\text { External } \\
\text { Rotation } \\
\text { Sequential Test }\end{array}$ & $\begin{array}{c}\text { External } \\
\text { Rotation } \\
\text { Simultaneous } \\
\text { Test } \\
\end{array}$ \\
\hline Spec\#3 L & 1.4 & 0.2 & 0.9 & 2.5 \\
\hline Spec\#5 L & 0.7 & 1.7 & 3.1 & 2.2 \\
\hline Spec\#6 L & 3.1 & 1.1 & 3.1 & 2.9 \\
\hline Spec\#9 L & 0.3 & 0.6 & 0.3 & 1.0 \\
\hline Spec\#13 R & 0.5 & 0.0 & 1.1 & 0.4 \\
\hline Spec\#15 L & 0.0 & 1.7 & 0.8 & 1.3 \\
\hline Mean & 1.0 & 0.9 & 1.6 & 1.7 \\
\hline Std & 1.1 & 0.7 & 1.2 & 1.0 \\
\hline
\end{tabular}


Table 5: Relative translation along $Z$ axis (harvested/native state) in camera frame

\begin{tabular}{|c|c|c|c|c|}
\hline $\begin{array}{l}\text { Harvested } \\
\text { (Screws) }\end{array}$ & $\begin{array}{c}\text { Internal } \\
\text { Rotation } \\
\text { Sequential Test }\end{array}$ & $\begin{array}{c}\text { Internal } \\
\text { Rotation } \\
\text { Simultaneous } \\
\text { Test }\end{array}$ & $\begin{array}{c}\text { External } \\
\text { Rotation } \\
\text { Sequential Test }\end{array}$ & $\begin{array}{c}\text { External } \\
\text { Rotation } \\
\text { Simultaneous } \\
\text { Test }\end{array}$ \\
\hline Spec\#1 R & 0.8 & 0.0 & 3.5 & 1.9 \\
\hline Spec\#2 L & 0.5 & 2.5 & 0.1 & 1.3 \\
\hline Spec\#4 R & 2.7 & 2.1 & 3.0 & 0.3 \\
\hline Spec\#14 R & 3.1 & 12.5 & 0.4 & 2.5 \\
\hline Spec\#17 R & 5.7 & 3.4 & 0.7 & 6.8 \\
\hline Spec\# 20 R & 0.9 & 4.0 & 4.4 & 3.1 \\
\hline Sepc\#21 R & 6.2 & 2.7 & 0.3 & 2.0 \\
\hline Mean & 2.8 & 3.9 & 1.8 & 2.6 \\
\hline Std & 2.3 & 4.0 & 1.8 & 2.1 \\
\hline $\begin{array}{c}\text { Harvested } \\
(\text { Plate }+ \text { Screw })\end{array}$ & $\begin{array}{c}\text { Internal } \\
\text { Rotation } \\
\text { Sequential Test }\end{array}$ & $\begin{array}{c}\text { Internal } \\
\text { Rotation } \\
\text { Simultaneous } \\
\text { Test }\end{array}$ & $\begin{array}{c}\text { External } \\
\text { Rotation } \\
\text { Sequential Test }\end{array}$ & $\begin{array}{c}\text { External } \\
\text { Rotation } \\
\text { Simultaneous } \\
\text { Test }\end{array}$ \\
\hline Spec\#3 L & 1.7 & 2.3 & 6.5 & 0.8 \\
\hline Spec\#5 L & 3.7 & 1.9 & 1.6 & 1.1 \\
\hline Spec\#6 L & 4.2 & 2.9 & 2.6 & 1.9 \\
\hline Spec\#9 L & 2.5 & 3.6 & 5.8 & 6.0 \\
\hline Spec\#13 R & 0.9 & 0.1 & 2.8 & 3.4 \\
\hline Spec\#15 L & 0.6 & 5.3 & 1.0 & 0.4 \\
\hline Mean & 2.3 & 2.7 & 3.4 & 2.3 \\
\hline Std & 1.5 & 1.7 & 2.3 & 2.1 \\
\hline
\end{tabular}


Table 6: Relative translation along $Z$ axis (instrumented state) in camera frame

\begin{tabular}{|c|c|c|c|c|}
\hline Screws & $\begin{array}{c}\text { Internal } \\
\text { Rotation } \\
\text { Sequential Test }\end{array}$ & $\begin{array}{c}\text { Internal } \\
\text { Rotation } \\
\text { Simultaneous } \\
\text { Test } \\
\end{array}$ & $\begin{array}{c}\text { External } \\
\text { Rotation } \\
\text { Sequential Test }\end{array}$ & $\begin{array}{c}\text { External } \\
\text { Rotation } \\
\text { Simultaneous } \\
\text { Test } \\
\end{array}$ \\
\hline Spec\#1 R & 6.4 & 5.2 & 2.1 & 1.7 \\
\hline Spec\#2 L & 1.7 & 0.8 & 5.1 & 3.3 \\
\hline Spec\#4 R & 2.1 & 0.3 & 4.7 & 1.9 \\
\hline Spec\#14 R & 3.8 & 1.1 & 3.7 & 0.8 \\
\hline Spec\#17 R & 0.8 & 3.3 & 1.7 & 7.8 \\
\hline Spec\# 20 R & 4.8 & 4.4 & 0.4 & 1.3 \\
\hline Sepc\#21 R & 2.3 & 6.7 & 1.4 & 2.9 \\
\hline Mean & 3.1 & 3.1 & 2.7 & 2.8 \\
\hline Std & 2.0 & 2.5 & 1.8 & 2.4 \\
\hline Plate + Screw & $\begin{array}{c}\text { Internal } \\
\text { Rotation } \\
\text { Sequential Test }\end{array}$ & $\begin{array}{c}\text { Internal } \\
\text { Rotation } \\
\text { Simultaneous } \\
\text { Test } \\
\end{array}$ & $\begin{array}{c}\text { External } \\
\text { Rotation } \\
\text { Sequential Test }\end{array}$ & $\begin{array}{c}\text { External } \\
\text { Rotation } \\
\text { Simultaneous } \\
\text { Test } \\
\end{array}$ \\
\hline Spec\#3 L & 6.7 & 5.3 & 3.8 & 0.8 \\
\hline Spec\#5 L & 0.6 & 0.7 & 4.6 & 2.6 \\
\hline Spec\#6 L & 2.9 & 2.0 & 2.1 & 1.7 \\
\hline Spec\#9 L & 1.2 & 0.8 & 0.6 & 8.6 \\
\hline Spec\#13 R & 1.3 & 0.2 & 6.3 & 2.6 \\
\hline Spec\#15 L & 2.0 & 0.2 & 3.0 & 4.7 \\
\hline Mean & 2.5 & 1.5 & 3.4 & 3.5 \\
\hline Std & 2.2 & 2.0 & 2.0 & 2.8 \\
\hline
\end{tabular}


Table 7: Relative rotation about $X$ axis (harvested/native state) in camera frame

\begin{tabular}{lcccc}
\hline $\begin{array}{c}\text { Harvested } \\
\text { (Screws) }\end{array}$ & $\begin{array}{c}\text { Internal } \\
\text { Rotation } \\
\text { Sequential Test }\end{array}$ & $\begin{array}{c}\text { Internal } \\
\text { Rotation } \\
\text { Simultaneous } \\
\text { Test }\end{array}$ & $\begin{array}{c}\text { External } \\
\text { Rotation } \\
\text { Sequential Test }\end{array}$ & $\begin{array}{c}\text { External } \\
\text { Rotation } \\
\text { Simultaneous } \\
\text { Test }\end{array}$ \\
\hline Spec\#1 R & 6.3 & 5.4 & 9.7 & 13.0 \\
Spec\#2 L & 1.1 & 0.4 & 4.2 & 5.1 \\
Spec\#4 R & 0.9 & 1.9 & 11.3 & 8.8 \\
Spec\#14 R & 3.3 & 8.4 & 9.2 & 13.1 \\
Spec\#17 R & 2.2 & 0.9 & 20.8 & 17.2 \\
Spec\#20 R & 1.3 & 3.6 & 7.6 & 6.8 \\
Sepc\#21 R & 0.3 & 1.9 & 3.0 & 1.1 \\
Mean & $\mathbf{2 . 2}$ & $\mathbf{3 . 2}$ & $\mathbf{9 . 4}$ & $\mathbf{9 . 3}$ \\
Std & $\mathbf{2 . 1}$ & $\mathbf{2 . 8}$ & $\mathbf{5 . 9}$ & $\mathbf{5 . 5}$ \\
& & & & \\
\hline & Internal & Internal & External & External \\
Harvested & Rotation & Rotation & Rotation & Rotation \\
(Plate + Screw) & Sequential Test & Simultaneous & Sequential Test & Test \\
\hline Spec\#3 L & 16.2 & 14.1 & 5.8 & 6.3 \\
Spec\#5 L & 2.6 & 0.9 & 1.8 & 1.3 \\
Spec\#6 L & 1.3 & 0.4 & 5.5 & 5.9 \\
Spec\#9 L & 0.8 & 1.0 & 5.0 & 5.2 \\
Spec\#13 R & 0.9 & 1.0 & 0.7 & 1.3 \\
Spec\#15 L & 2.7 & 1.4 & 2.9 & 3.1 \\
Mean & $\mathbf{4 . 1}$ & $\mathbf{3 . 1}$ & $\mathbf{3 . 6}$ & $\mathbf{3 . 9}$ \\
Std & $\mathbf{6 . 0}$ & $\mathbf{5 . 4}$ & $\mathbf{2 . 1}$ & $\mathbf{2 . 3}$ \\
\hline
\end{tabular}


Table 8: Relative rotation about $X$ axis (instrumented state) in camera frame

\begin{tabular}{lcccc}
\hline \multicolumn{1}{c}{ Screws } & $\begin{array}{c}\text { Internal } \\
\text { Rotation } \\
\text { Sequential Test }\end{array}$ & $\begin{array}{c}\text { Internal } \\
\text { Rotation } \\
\text { Simultaneous } \\
\text { Test }\end{array}$ & $\begin{array}{c}\text { External } \\
\text { Rotation } \\
\text { Sequential Test }\end{array}$ & $\begin{array}{c}\text { External } \\
\text { Rotation } \\
\text { Simultaneous } \\
\text { Test }\end{array}$ \\
\hline Spec\#1 R & 2.4 & 8.5 & 8.4 & 7.9 \\
Spec\#2 L & 0.0 & 0.5 & 5.9 & 6.4 \\
Spec\#4 R & 0.2 & 1.9 & 1.1 & 5.3 \\
Spec\#14 R & 1.6 & 0.2 & 5.4 & 2.9 \\
Spec\#17 R & 0.1 & 3.7 & 1.6 & 3.1 \\
Spec\# 20 R & 3.4 & 2.4 & 4.3 & 5.2 \\
Sepc\#21 R & 1.3 & 1.9 & 0.1 & 0.5 \\
Mean & $\mathbf{1 . 3}$ & $\mathbf{2 . 7}$ & $\mathbf{3 . 8}$ & $\mathbf{4 . 5}$ \\
Std & $\mathbf{1 . 3}$ & $\mathbf{2 . 8}$ & $\mathbf{3 . 0}$ & $\mathbf{2 . 5}$ \\
& & & & \\
\hline & Internal & Internal & External & External \\
Plate + Screw & Rotation & Rotation & Rotation & Rotation \\
& Sequential Test & Simultaneous & Sequential Test & Simultaneous \\
\hline Spec\#3 L & 3.5 & Test & 2.2 & Test \\
Spec\#5 L & 0.3 & 2.7 & 4.6 & 4.8 \\
Spec\#6 L & 3.2 & 0.2 & 5.4 & 4.2 \\
Spec\#9 L & 1.5 & 1.7 & 3.0 & 5.9 \\
Spec\#13 R & 3.4 & 0.8 & 2.5 & 1.8 \\
Spec\#15 L & 0.6 & 2.7 & $\mathbf{3 . 8}$ & 0.8 \\
Mean & $\mathbf{2 . 1}$ & 0.2 & $\mathbf{1 . 4}$ & $\mathbf{3 . 8}$ \\
Std & $\mathbf{1 . 5}$ & $\mathbf{1 . 2}$ & & $\mathbf{2 . 1}$ \\
\hline & & & & \\
\hline
\end{tabular}


Table 9: Relative rotation about $Y$ axis (harvested/native state) in camera frame

\begin{tabular}{lcccc}
\hline $\begin{array}{c}\text { Harvested } \\
\text { (Screws) }\end{array}$ & $\begin{array}{c}\text { Internal } \\
\text { Rotation } \\
\text { Sequential Test }\end{array}$ & $\begin{array}{c}\text { Internal } \\
\text { Rotation } \\
\text { Simultaneous } \\
\text { Test }\end{array}$ & $\begin{array}{c}\text { External } \\
\text { Rotation } \\
\text { Sequential Test }\end{array}$ & $\begin{array}{c}\text { External } \\
\text { Rotation } \\
\text { Simultaneous } \\
\text { Test }\end{array}$ \\
\hline Spec\#1 R & 4.9 & 3.5 & 10.9 & 10.5 \\
Spec\#2 L & 1.1 & 1.9 & 8.7 & 9.5 \\
Spec\#4 R & 3.2 & 4.4 & 10.0 & 10.2 \\
Spec\#14 R & 3.2 & 1.2 & 5.0 & 7.1 \\
Spec\#17 R & 9.0 & 4.4 & 7.6 & 3.5 \\
Spec\# 20 R & 9.0 & 4.9 & 7.9 & 4.7 \\
Sepc\#21 R & 4.8 & 4.9 & 11.7 & 9.5 \\
Mean & $\mathbf{5 . 0}$ & $\mathbf{3 . 6}$ & $\mathbf{8 . 8}$ & $\mathbf{7 . 9}$ \\
Std & $\mathbf{3 . 0}$ & $\mathbf{1 . 5}$ & $\mathbf{2 . 2}$ & $\mathbf{2 . 8}$ \\
& & & & \\
\hline & Internal & Internal & External & External \\
Harvested & Rotation & Rotation & Rotation & Rotation \\
(Plate+ Screw) & Sequential Test & Simultaneous & Sequential Test & Simultaneous \\
& & Test & 10.3 & Test \\
\hline Spec\#3 L & 0.4 & 0.6 & 8.0 & 5.2 \\
Spec\#5 L & 4.0 & 2.7 & 10.8 & 9.7 \\
Spec\#6 L & 0.2 & 1.2 & 10.8 & 9.2 \\
Spec\#9 L & 12.3 & 10.9 & 6.9 & 12.6 \\
Spec\#13 R & 1.3 & 2.4 & $\mathbf{8 . 0}$ & 5.8 \\
Spec\#15 L & 2.4 & 2.8 & $\mathbf{3 . 7}$ & $\mathbf{7 . 5}$ \\
Mean & $\mathbf{3 . 4}$ & $\mathbf{3 . 4}$ & & $\mathbf{3 . 6}$ \\
Std & $\mathbf{4 . 6}$ & $\mathbf{3 . 8}$ & & \\
\hline
\end{tabular}


Table 10: Relative rotation about $Y$ axis (instrumented state) in camera frame

\begin{tabular}{lcccc}
\hline \multicolumn{1}{c}{ Screws } & $\begin{array}{c}\text { Internal } \\
\text { Rotation } \\
\text { Sequential Test }\end{array}$ & $\begin{array}{c}\text { Internal } \\
\text { Rotation } \\
\text { Simultaneous } \\
\text { Test }\end{array}$ & $\begin{array}{c}\text { External } \\
\text { Rotation } \\
\text { Sequential Test }\end{array}$ & $\begin{array}{c}\text { External } \\
\text { Rotation } \\
\text { Simultaneous } \\
\text { Test }\end{array}$ \\
\hline Spec\#1 R & 4.1 & 1.5 & 2.9 & 1.6 \\
Spec\#2 L & 2.1 & 1.3 & 3.7 & 4.3 \\
Spec\#4 R & 3.9 & 2.8 & 4.9 & 4.8 \\
Spec\#14 R & 1.0 & 0.2 & 1.4 & 1.6 \\
Spec\#17 R & 1.8 & 3.6 & 0.0 & 0.0 \\
Spec\# 20 R & 1.2 & 3.5 & 4.5 & 6.1 \\
Sepc\#21 R & 6.5 & 4.6 & 7.3 & 6.3 \\
Mean & $\mathbf{2 . 9}$ & $\mathbf{2 . 5}$ & $\mathbf{3 . 5}$ & $\mathbf{3 . 5}$ \\
Std & $\mathbf{2 . 0}$ & $\mathbf{1 . 6}$ & $\mathbf{2 . 4}$ & $\mathbf{2 . 5}$ \\
& & & & \\
\hline & Internal & Internal & External & External \\
Plate + Screw & Rotation & Rotation & Rotation & Rotation \\
& Sequential Test & Simultaneous & Sequential Test & Simultaneous \\
\hline Spec\#3 L & 2.6 & Test & 0.9 & Test \\
Spec\#5 L & 0.1 & 3.7 & 3.2 & 0.7 \\
Spec\#6 L & 1.3 & 2.6 & 1.5 & 3.7 \\
Spec\#9 L & 1.8 & 0.7 & 0.9 & 0.4 \\
Spec\#13 R & 4.1 & 3.8 & 1.4 & 0.8 \\
Spec\#15 L & 0.1 & 4.4 & $\mathbf{1 . 7}$ & 3.2 \\
Mean & $\mathbf{1 . 6}$ & 1.4 & $\mathbf{0 . 9}$ & $\mathbf{1 . 9}$ \\
Std & $\mathbf{1 . 5}$ & $\mathbf{1 . 5}$ & & $\mathbf{1 . 5}$ \\
\hline & & & & \\
\hline
\end{tabular}


Table 11: Relative rotation about $Z$ axis (harvested/native state) in camera frame

\begin{tabular}{lcccc}
\hline $\begin{array}{c}\text { Harvested } \\
\text { (Screws) }\end{array}$ & $\begin{array}{c}\text { Internal } \\
\text { Rotation } \\
\text { Sequential Test }\end{array}$ & $\begin{array}{c}\text { Internal } \\
\text { Rotation } \\
\text { Simultaneous } \\
\text { Test }\end{array}$ & $\begin{array}{c}\text { External } \\
\text { Rotation } \\
\text { Sequential Test }\end{array}$ & $\begin{array}{c}\text { External } \\
\text { Rotation } \\
\text { Simultaneous } \\
\text { Test }\end{array}$ \\
\hline Spec\#1 R & 0.2 & 1.4 & 5.9 & 5.1 \\
Spec\#2 L & 1.6 & 2.1 & 2.2 & 1.2 \\
Spec\#4 R & 1.4 & 2.3 & 5.0 & 5.4 \\
Spec\#14 R & 0.1 & 0.2 & 3.8 & 2.6 \\
Spec\#17 R & 1.7 & 0.1 & 11.7 & 12.4 \\
Spec\# 20 R & 0.8 & 0.8 & 5.8 & 5.8 \\
Sepc\#21 R & 0.3 & 0.0 & 3.7 & 4.1 \\
Mean & $\mathbf{0 . 9}$ & $\mathbf{1 . 0}$ & $\mathbf{5 . 4}$ & $\mathbf{5 . 2}$ \\
Std & $\mathbf{0 . 7}$ & $\mathbf{0 . 9}$ & $\mathbf{3 . 1}$ & $\mathbf{3 . 6}$ \\
& & & & \\
\hline & Internal & Internal & External & External \\
Harvested & Rotation & Rotation & Rotation & Rotation \\
(Plate + Screw) & Sequential Test & Simultaneous & Sequential Test & Test \\
\hline Spec\#3 L & 7.1 & 7.6 & 0.4 & 1.2 \\
Spec\#5 L & 2.1 & 0.3 & 3.0 & 2.9 \\
Spec\#6 L & 2.1 & 3.4 & 2.5 & 3.2 \\
Spec\#9 L & 2.1 & 2.4 & 3.7 & 5.7 \\
Spec\#13 R & 0.1 & 0.5 & 4.8 & 4.7 \\
Spec\#15 L & 0.7 & 1.2 & 3.6 & 3.6 \\
Mean & $\mathbf{2 . 4}$ & $\mathbf{2 . 5}$ & $\mathbf{3 . 0}$ & $\mathbf{3 . 5}$ \\
Std & $\mathbf{2 . 5}$ & $\mathbf{2 . 7}$ & $\mathbf{1 . 5}$ & \\
\hline
\end{tabular}


Table 12: Relative rotation about $Z$ axis (instrumented state) in camera frame

\begin{tabular}{lcccc}
\hline \multicolumn{1}{c}{ Screws } & $\begin{array}{c}\text { Internal } \\
\text { Rotation } \\
\text { Sequential Test }\end{array}$ & $\begin{array}{c}\text { Internal } \\
\text { Rotation } \\
\text { Simultaneous } \\
\text { Test }\end{array}$ & $\begin{array}{c}\text { External } \\
\text { Rotation } \\
\text { Sequential Test }\end{array}$ & $\begin{array}{c}\text { External } \\
\text { Rotation } \\
\text { Simultaneous } \\
\text { Test }\end{array}$ \\
\hline Spec\#1 R & 1.0 & 1.2 & 3.7 & 3.6 \\
Spec\#2 L & 1.8 & 1.4 & 2.9 & 3.4 \\
Spec\#4 R & 2.0 & 2.2 & 1.0 & 2.4 \\
Spec\#14 R & 1.5 & 1.4 & 1.0 & 0.7 \\
Spec\#17 R & 0.6 & 2.5 & 2.4 & 4.4 \\
Spec\# 20 R & 0.6 & 0.9 & 1.9 & 2.1 \\
Sepc\#21 R & 0.9 & 1.3 & 4.7 & 4.4 \\
Mean & $\mathbf{1 . 2}$ & $\mathbf{1 . 6}$ & $\mathbf{2 . 5}$ & $\mathbf{3 . 0}$ \\
Std & $\mathbf{0 . 6}$ & $\mathbf{0 . 6}$ & $\mathbf{1 . 4}$ & $\mathbf{1 . 4}$ \\
& & & & \\
\hline & Internal & Internal & External & External \\
Plate + Screw & Rotation & Rotation & Rotation & Rotation \\
& Sequential Test & Simultaneous & Sequential Test & Simultaneous \\
\hline Spec\#3 L & 0.6 & Test & 0.1 & Test \\
Spec\#5 L & 1.1 & 0.5 & 0.0 & 0.3 \\
Spec\#6 L & 0.1 & 0.9 & 0.1 & 0.7 \\
Spec\#9 L & 0.4 & 0.6 & 1.2 & 0.5 \\
Spec\#13 R & 2.8 & 0.6 & 0.1 & 0.8 \\
Spec\#15 L & 0.6 & 2.8 & 0.7 & 0.9 \\
Mean & $\mathbf{0 . 9}$ & 0.2 & $\mathbf{0 . 5}$ & 0.8 \\
Std & $\mathbf{1 . 0}$ & $\mathbf{0 . 9}$ & & $\mathbf{0 . 7}$ \\
\hline & & & & $\mathbf{0 . 2}$ \\
\hline
\end{tabular}


Table 13: Relative translation along $X^{\prime}$ axis (harvested/native state) in joint frame

\begin{tabular}{|c|c|c|c|c|}
\hline $\begin{array}{l}\text { Harvested } \\
\text { (Screws) }\end{array}$ & $\begin{array}{c}\text { Internal } \\
\text { Rotation } \\
\text { Sequential Test }\end{array}$ & $\begin{array}{c}\text { Internal } \\
\text { Rotation } \\
\text { Simultaneous } \\
\text { Test }\end{array}$ & $\begin{array}{c}\text { External } \\
\text { Rotation } \\
\text { Sequential Test }\end{array}$ & $\begin{array}{c}\text { External } \\
\text { Rotation } \\
\text { Simultaneous } \\
\text { Test }\end{array}$ \\
\hline Spec\#1 R & 1.8 & 1.7 & 1.2 & 0.4 \\
\hline Spec\#2 L & 0.4 & 1.0 & 1.5 & 0.7 \\
\hline Spec\#4 R & 0.2 & 2.0 & 3.3 & 2.3 \\
\hline Spec\#14 R & 0.5 & 2.7 & 2.0 & 5.1 \\
\hline Spec\#17 R & 2.2 & 1.0 & 1.1 & 1.6 \\
\hline Spec\# 20 R & 2.4 & 1.7 & 2.4 & 0.2 \\
\hline Sepc\#21 R & 1.0 & 0.1 & 0.1 & 0.1 \\
\hline Mean & 1.2 & 1.5 & 1.7 & 1.5 \\
\hline Std & 0.9 & 0.8 & 1.0 & 1.8 \\
\hline $\begin{array}{c}\text { Harvested } \\
(\text { Plate }+ \text { Screw })\end{array}$ & $\begin{array}{c}\text { Internal } \\
\text { Rotation } \\
\text { Sequential Test }\end{array}$ & $\begin{array}{c}\text { Internal } \\
\text { Rotation } \\
\text { Simultaneous } \\
\text { Test } \\
\end{array}$ & $\begin{array}{c}\text { External } \\
\text { Rotation } \\
\text { Sequential Test }\end{array}$ & $\begin{array}{c}\text { External } \\
\text { Rotation } \\
\text { Simultaneous } \\
\text { Test } \\
\end{array}$ \\
\hline Spec\#3 L & 0.1 & 2.9 & 0.9 & 3.3 \\
\hline Spec\#5 L & 3.8 & 1.8 & 1.9 & 2.4 \\
\hline Spec\#6 L & 4.8 & 2.5 & 2.0 & 1.2 \\
\hline Spec\#9 L & 5.9 & 5.8 & 2.1 & 5.7 \\
\hline Spec\#13 R & 0.3 & 0.8 & 1.3 & 0.5 \\
\hline Spec\#15 L & 1.4 & 3.7 & 0.4 & 0.3 \\
\hline Mean & 1.8 & 2.0 & 1.5 & 1.9 \\
\hline Std & 1.8 & 1.5 & 0.8 & 1.8 \\
\hline
\end{tabular}


Table 14: Relative translation along $X^{\prime}$ axis (instrumented state) in joint frame

\begin{tabular}{|c|c|c|c|c|}
\hline Screws & $\begin{array}{c}\text { Internal } \\
\text { Rotation } \\
\text { Sequential Test }\end{array}$ & $\begin{array}{c}\text { Internal } \\
\text { Rotation } \\
\text { Simultaneous } \\
\text { Test } \\
\end{array}$ & $\begin{array}{c}\text { External } \\
\text { Rotation } \\
\text { Sequential Test }\end{array}$ & $\begin{array}{c}\text { External } \\
\text { Rotation } \\
\text { Simultaneous } \\
\text { Test } \\
\end{array}$ \\
\hline Spec\#1 R & 4.6 & 1.3 & 2.1 & 2.4 \\
\hline Spec\#2 L & 2.5 & 1.1 & 2.0 & 2.3 \\
\hline Spec\#4 R & 0.8 & 0.1 & 3.1 & 1.3 \\
\hline Spec\#14 R & 2.1 & 1.8 & 0.1 & 0.1 \\
\hline Spec\#17 R & 2.5 & 2.3 & 1.8 & 1.8 \\
\hline Spec\# 20 R & 1.5 & 0.2 & 1.5 & 2.8 \\
\hline Sepc\#21 R & 1.0 & 0.5 & 1.6 & 1.4 \\
\hline Mean & 2.1 & 1.0 & 1.7 & 1.7 \\
\hline Std & 1.3 & 0.8 & 0.9 & 0.9 \\
\hline Plate + Screw & $\begin{array}{c}\text { Internal } \\
\text { Rotation } \\
\text { Sequential Test }\end{array}$ & $\begin{array}{c}\text { Internal } \\
\text { Rotation } \\
\text { Simultaneous } \\
\text { Test } \\
\end{array}$ & $\begin{array}{c}\text { External } \\
\text { Rotation } \\
\text { Sequential Test }\end{array}$ & $\begin{array}{c}\text { External } \\
\text { Rotation } \\
\text { Simultaneous } \\
\text { Test } \\
\end{array}$ \\
\hline Spec\#3 L & 1.6 & 1.7 & 0.6 & 2.5 \\
\hline Spec\#5 L & 0.4 & 2.6 & 3.1 & 1.3 \\
\hline Spec\#6 L & 1.4 & 1.0 & 0.6 & 0.1 \\
\hline Spec\#9 L & 1.2 & 1.6 & 0.7 & 0.6 \\
\hline Spec\#13 R & 0.4 & 0.2 & 0.6 & 0.9 \\
\hline Spec\#15 L & 0.2 & 1.2 & 0.7 & 0.1 \\
\hline Mean & 0.9 & 1.4 & 1.1 & 0.9 \\
\hline Std & 0.6 & 0.8 & 1.0 & 0.9 \\
\hline
\end{tabular}


Table 15: Relative translation along $Y^{\prime}$ axis (harvested/native state) in joint frame

\begin{tabular}{|c|c|c|c|c|}
\hline $\begin{array}{l}\text { Harvested } \\
\text { (Screws) }\end{array}$ & $\begin{array}{c}\text { Internal } \\
\text { Rotation } \\
\text { Sequential Test }\end{array}$ & $\begin{array}{c}\text { Internal } \\
\text { Rotation } \\
\text { Simultaneous } \\
\text { Test } \\
\end{array}$ & $\begin{array}{c}\text { External } \\
\text { Rotation } \\
\text { Sequential Test }\end{array}$ & $\begin{array}{c}\text { External } \\
\text { Rotation } \\
\text { Simultaneous } \\
\text { Test } \\
\end{array}$ \\
\hline Spec\#1 R & 0.8 & 1.5 & 0.4 & 2.3 \\
\hline Spec\#2 L & 0.1 & 0.2 & 0.4 & 0.3 \\
\hline Spec\#4 R & 0.8 & 1.9 & 1.0 & 1.0 \\
\hline Spec\#14 R & 0.7 & 0.4 & 0.4 & 0.6 \\
\hline Spec\#17 R & 2.0 & 1.4 & 1.3 & 1.8 \\
\hline Spec\# 20 R & 0.9 & 1.4 & 0.1 & 0.6 \\
\hline Sepc\#21 R & 0.8 & 0.0 & 0.8 & 0.7 \\
\hline Mean & 0.9 & 1.0 & 0.6 & 1.0 \\
\hline Std & 0.6 & 0.7 & 0.4 & 0.7 \\
\hline $\begin{array}{c}\text { Harvested } \\
(\text { Plate }+ \text { Screw })\end{array}$ & $\begin{array}{c}\text { Internal } \\
\text { Rotation } \\
\text { Sequential Test }\end{array}$ & $\begin{array}{c}\text { Internal } \\
\text { Rotation } \\
\text { Simultaneous } \\
\text { Test }\end{array}$ & $\begin{array}{c}\text { External } \\
\text { Rotation } \\
\text { Sequential Test }\end{array}$ & $\begin{array}{c}\text { External } \\
\text { Rotation } \\
\text { Simultaneous } \\
\text { Test }\end{array}$ \\
\hline Spec\#3 L & 0.8 & 0.2 & 1.6 & 1.6 \\
\hline Spec\#5 L & 0.1 & 0.1 & 2.9 & 2.9 \\
\hline Spec\#6 L & 0.5 & 0.4 & 1.2 & 1.1 \\
\hline Spec\#9 L & 2.7 & 1.9 & 0.8 & 2.1 \\
\hline Spec\#13 R & 1.0 & 1.3 & 0.7 & 0.2 \\
\hline Spec\#15 L & 0.5 & 0.5 & 0.5 & 0.8 \\
\hline Mean & 0.9 & 0.8 & 0.9 & 1.1 \\
\hline Std & 0.7 & 0.7 & 0.7 & 0.8 \\
\hline
\end{tabular}


Table 16: Relative translation along $Y^{\prime}$ axis (instrumented state) in joint frame

\begin{tabular}{lcccc}
\hline \multicolumn{1}{c}{ Screws } & $\begin{array}{c}\text { Internal } \\
\text { Rotation } \\
\text { Sequential Test }\end{array}$ & $\begin{array}{c}\text { Internal } \\
\text { Rotation } \\
\text { Simultaneous } \\
\text { Test }\end{array}$ & $\begin{array}{c}\text { External } \\
\text { Rotation } \\
\text { Sequential Test }\end{array}$ & $\begin{array}{c}\text { External } \\
\text { Rotation } \\
\text { Simultaneous } \\
\text { Test }\end{array}$ \\
\hline Spec\#1 R & 0.2 & 2.4 & 0.8 & 0.8 \\
Spec\#2 L & 1.4 & 1.1 & 3.2 & 3.4 \\
Spec\#4 R & 0.2 & 0.5 & 1.2 & 0.8 \\
Spec\#14 R & 0.6 & 0.1 & 0.6 & 0.3 \\
Spec\#17 R & 0.4 & 1.1 & 0.6 & 2.3 \\
Spec\# 20 R & 0.5 & 0.1 & 1.3 & 1.8 \\
Sepc\#21 R & 0.3 & 0.8 & 0.4 & 0.4 \\
Mean & $\mathbf{0 . 5}$ & $\mathbf{0 . 9}$ & $\mathbf{1 . 2}$ & $\mathbf{1 . 4}$ \\
Std & $\mathbf{0 . 4}$ & $\mathbf{0 . 8}$ & $\mathbf{1 . 0}$ & $\mathbf{1 . 1}$ \\
& & & & External \\
Plate + Screw & Rotation & Rotation & External & Rotation \\
& Sequential Test & Simultaneous & Rotation & Simultaneous \\
\hline Spec\#3 L & 0.6 & 1.0 & 0.6 & Test \\
Spec\#5 L & 0.6 & 0.5 & 1.8 & 1.3 \\
Spec\#6 L & 2.7 & 2.0 & 3.3 & 1.8 \\
Spec\#9 L & 0.3 & 0.2 & 0.0 & 3.6 \\
Spec\#13 R & 0.7 & 0.1 & 1.0 & 1.4 \\
Spec\#15 L & 0.2 & 1.2 & 1.7 & 0.1 \\
Mean & $\mathbf{0 . 9}$ & $\mathbf{0 . 8}$ & $\mathbf{1 . 4}$ & 1.7 \\
Std & $\mathbf{0 . 9}$ & $\mathbf{0 . 7}$ & & $\mathbf{1 . 1}$ \\
\hline
\end{tabular}


Table 17: Relative translation along $Z^{\prime}$ axis (harvested/native state) in joint frame

\begin{tabular}{|c|c|c|c|c|}
\hline $\begin{array}{l}\text { Harvested } \\
\text { (Screws) }\end{array}$ & $\begin{array}{c}\text { Internal } \\
\text { Rotation } \\
\text { Sequential Test }\end{array}$ & $\begin{array}{c}\text { Internal } \\
\text { Rotation } \\
\text { Simultaneous } \\
\text { Test }\end{array}$ & $\begin{array}{c}\text { External } \\
\text { Rotation } \\
\text { Sequential Test }\end{array}$ & $\begin{array}{c}\text { External } \\
\text { Rotation } \\
\text { Simultaneous } \\
\text { Test }\end{array}$ \\
\hline Spec\#1 R & 1.0 & 0.1 & 3.7 & 2.3 \\
\hline Spec\#2 L & 0.4 & 2.3 & 0.1 & 1.2 \\
\hline Spec\#4 R & 2.7 & 2.0 & 3.2 & 0.2 \\
\hline Spec\#14 R & 3.1 & 12.5 & 0.4 & 2.6 \\
\hline Spec\#17 R & 5.6 & 3.4 & 0.7 & 6.8 \\
\hline Spec\# 20 R & 1.0 & 4.0 & 4.3 & 3.1 \\
\hline Sepc\#21 R & 6.1 & 2.7 & 0.2 & 2.0 \\
\hline Mean & 2.8 & 3.9 & 1.8 & 2.6 \\
\hline Std & 2.3 & 4.0 & 1.8 & 2.1 \\
\hline $\begin{array}{c}\text { Harvested } \\
(\text { Plate }+ \text { Screw })\end{array}$ & $\begin{array}{c}\text { Internal } \\
\text { Rotation } \\
\text { Sequential Test }\end{array}$ & $\begin{array}{c}\text { Internal } \\
\text { Rotation } \\
\text { Simultaneous } \\
\text { Test } \\
\end{array}$ & $\begin{array}{c}\text { External } \\
\text { Rotation } \\
\text { Sequential Test }\end{array}$ & $\begin{array}{c}\text { External } \\
\text { Rotation } \\
\text { Simultaneous } \\
\text { Test }\end{array}$ \\
\hline Spec\#3 L & 1.7 & 1.4 & 6.4 & 0.1 \\
\hline Spec\#5 L & 2.9 & 1.5 & 2.5 & 2.2 \\
\hline Spec\#6 L & 3.9 & 2.7 & 2.4 & 1.8 \\
\hline Spec\#9 L & 2.5 & 3.6 & 5.8 & 6.0 \\
\hline Spec\#13 R & 0.9 & 0.1 & 2.8 & 3.4 \\
\hline Spec\#15 L & 0.3 & 4.9 & 1.0 & 0.2 \\
\hline Mean & 2.6 & 3.5 & 2.4 & 2.4 \\
\hline Std & 1.8 & 2.9 & 2.0 & 2.0 \\
\hline
\end{tabular}


Table 18: Relative translation along $Z^{\prime}$ axis (instrumented state) in joint frame

\begin{tabular}{|c|c|c|c|c|}
\hline Screws & $\begin{array}{c}\text { Internal } \\
\text { Rotation } \\
\text { Sequential Test }\end{array}$ & $\begin{array}{c}\text { Internal } \\
\text { Rotation } \\
\text { Simultaneous } \\
\text { Test } \\
\end{array}$ & $\begin{array}{c}\text { External } \\
\text { Rotation } \\
\text { Sequential Test }\end{array}$ & $\begin{array}{c}\text { External } \\
\text { Rotation } \\
\text { Simultaneous } \\
\text { Test } \\
\end{array}$ \\
\hline Spec\#1 R & 5.8 & 5.3 & 1.7 & 2.3 \\
\hline Spec\#2 L & 1.5 & 0.7 & 5.1 & 3.3 \\
\hline Spec\#4 R & 2.1 & 0.3 & 4.5 & 2.0 \\
\hline Spec\#14 R & 3.7 & 1.0 & 3.7 & 0.8 \\
\hline Spec\#17 R & 0.7 & 3.3 & 1.8 & 7.8 \\
\hline Spec\# 20 R & 4.9 & 4.4 & 0.6 & 1.5 \\
\hline Sepc\#21 R & 2.2 & 6.7 & 1.4 & 2.9 \\
\hline Mean & 3.0 & 3.1 & 2.7 & 2.9 \\
\hline Std & 1.9 & 2.5 & 1.7 & 2.3 \\
\hline Plate + Screw & $\begin{array}{c}\text { Internal } \\
\text { Rotation } \\
\text { Sequential Test }\end{array}$ & $\begin{array}{c}\text { Internal } \\
\text { Rotation } \\
\text { Simultaneous } \\
\text { Test } \\
\end{array}$ & $\begin{array}{c}\text { External } \\
\text { Rotation } \\
\text { Sequential Test }\end{array}$ & $\begin{array}{c}\text { External } \\
\text { Rotation } \\
\text { Simultaneous } \\
\text { Test } \\
\end{array}$ \\
\hline Spec\#3 L & 7.8 & 4.9 & 4.2 & 0.3 \\
\hline Spec\#5 L & 0.7 & 0.1 & 4.2 & 2.6 \\
\hline Spec\#6 L & 2.9 & 2.2 & 2.2 & 1.9 \\
\hline Spec\#9 L & 1.2 & 0.8 & 0.6 & 8.6 \\
\hline Spec\#13 R & 1.3 & 0.2 & 6.3 & 2.6 \\
\hline Spec\#15 L & 2.0 & 0.2 & 3.5 & 5.1 \\
\hline Mean & 2.7 & 1.4 & 3.5 & 3.5 \\
\hline Std & 2.6 & 1.9 & 1.9 & 2.9 \\
\hline
\end{tabular}


Table 19: Relative rotation about $X^{\prime}$ axis (harvested/native state) in joint frame

\begin{tabular}{|c|c|c|c|c|}
\hline $\begin{array}{l}\text { Harvested } \\
\text { (Screws) }\end{array}$ & $\begin{array}{c}\text { Internal } \\
\text { Rotation } \\
\text { Sequential Test }\end{array}$ & $\begin{array}{c}\text { Internal } \\
\text { Rotation } \\
\text { Simultaneous } \\
\text { Test } \\
\end{array}$ & $\begin{array}{c}\text { External } \\
\text { Rotation } \\
\text { Sequential Test }\end{array}$ & $\begin{array}{c}\text { External } \\
\text { Rotation } \\
\text { Simultaneous } \\
\text { Test } \\
\end{array}$ \\
\hline Spec\#1 R & 2.2 & 2.6 & 0.2 & 3.2 \\
\hline Spec\#2 L & 1.1 & 0.8 & 0.0 & 0.3 \\
\hline Spec\#4 R & 2.4 & 0.7 & 4.1 & 1.9 \\
\hline Spec\#14 R & 4.4 & 8.2 & 6.5 & 9.1 \\
\hline Spec\#17 R & 2.9 & 1.6 & 21.3 & 16.0 \\
\hline Spec\#20 R & 4.3 & 0.1 & 1.1 & 2.3 \\
\hline Sepc\#21 R & 1.6 & 0.2 & 1.8 & 2.6 \\
\hline Mean & 2.7 & 2.0 & 5.0 & 5.1 \\
\hline Std & 1.2 & 2.8 & 7.5 & 5.6 \\
\hline $\begin{array}{c}\text { Harvested } \\
(\text { Plate }+ \text { Screw })\end{array}$ & $\begin{array}{c}\text { Internal } \\
\text { Rotation } \\
\text { Sequential Test }\end{array}$ & $\begin{array}{c}\text { Internal } \\
\text { Rotation } \\
\text { Simultaneous } \\
\text { Test } \\
\end{array}$ & $\begin{array}{c}\text { External } \\
\text { Rotation } \\
\text { Sequential Test }\end{array}$ & $\begin{array}{c}\text { External } \\
\text { Rotation } \\
\text { Simultaneous } \\
\text { Test } \\
\end{array}$ \\
\hline Spec\#3 L & 15.1 & 13.2 & 1.4 & 1.3 \\
\hline Spec\#5 L & 0.9 & 0.4 & 4.4 & 4.7 \\
\hline Spec\#6 L & 0.7 & 0.6 & 1.2 & 0.1 \\
\hline Spec\#9 L & 6.5 & 6.0 & 0.7 & 1.3 \\
\hline Spec\#13 R & 1.3 & 1.8 & 3.4 & 3.6 \\
\hline Spec\#15 L & 3.7 & 1.2 & 2.1 & 1.2 \\
\hline Mean & 4.7 & 3.9 & 2.2 & 2.0 \\
\hline Std & 5.5 & 5.0 & 1.4 & 1.7 \\
\hline
\end{tabular}


Table 20: Relative rotation about $X^{\prime}$ axis (instrumented state) in joint frame

\begin{tabular}{|c|c|c|c|c|}
\hline Screws & $\begin{array}{c}\text { Internal } \\
\text { Rotation } \\
\text { Sequential Test }\end{array}$ & $\begin{array}{c}\text { Internal } \\
\text { Rotation } \\
\text { Simultaneous } \\
\text { Test } \\
\end{array}$ & $\begin{array}{c}\text { External } \\
\text { Rotation } \\
\text { Sequential Test }\end{array}$ & $\begin{array}{c}\text { External } \\
\text { Rotation } \\
\text { Simultaneous } \\
\text { Test } \\
\end{array}$ \\
\hline Spec\#1 R & 0.2 & 6.1 & 4.2 & 4.6 \\
\hline Spec\#2 L & 0.6 & 0.8 & 3.9 & 4.2 \\
\hline Spec\#4 R & 2.2 & 3.0 & 1.7 & 1.8 \\
\hline Spec\#14 R & 1.1 & 0.3 & 4.4 & 2.0 \\
\hline Spec\#17 R & 1.1 & 1.2 & 1.4 & 2.5 \\
\hline Spec\# 20 R & 3.4 & 3.9 & 0.7 & 0.5 \\
\hline Sepc\#21 R & 1.4 & 3.6 & 2.6 & 2.9 \\
\hline Mean & 1.4 & 2.7 & 2.7 & 2.6 \\
\hline Std & 1.1 & 2.1 & 1.5 & 1.4 \\
\hline Plate + Screw & $\begin{array}{c}\text { Internal } \\
\text { Rotation } \\
\text { Sequential Test }\end{array}$ & $\begin{array}{c}\text { Internal } \\
\text { Rotation } \\
\text { Simultaneous } \\
\text { Test } \\
\end{array}$ & $\begin{array}{c}\text { External } \\
\text { Rotation } \\
\text { Sequential Test }\end{array}$ & $\begin{array}{c}\text { External } \\
\text { Rotation } \\
\text { Simultaneous } \\
\text { Test } \\
\end{array}$ \\
\hline Spec\#3 L & 0.9 & 0.3 & 1.1 & 3.0 \\
\hline Spec\#5 L & 0.1 & 0.7 & 2.4 & 1.6 \\
\hline Spec\#6 L & 1.9 & 1.9 & 3.6 & 4.8 \\
\hline Spec\#9 L & 0.5 & 1.1 & 3.1 & 2.0 \\
\hline Spec\#13 R & 4.6 & 4.0 & 2.8 & 0.4 \\
\hline Spec\#15 L & 0.4 & 0.8 & 1.6 & 1.6 \\
\hline Mean & 1.4 & 1.5 & 2.4 & 2.2 \\
\hline Std & 1.7 & 1.4 & 0.9 & 1.5 \\
\hline
\end{tabular}


Table 21: Relative rotation about $Y^{\prime}$ axis (harvested/native state) in joint frame

\begin{tabular}{lcccc}
\hline $\begin{array}{c}\text { Harvested } \\
\text { (Screws) }\end{array}$ & $\begin{array}{c}\text { Internal } \\
\text { Rotation } \\
\text { Sequential Test }\end{array}$ & $\begin{array}{c}\text { Internal } \\
\text { Rotation } \\
\text { Simultaneous } \\
\text { Test }\end{array}$ & $\begin{array}{c}\text { External } \\
\text { Rotation } \\
\text { Sequential Test }\end{array}$ & $\begin{array}{c}\text { External } \\
\text { Rotation } \\
\text { Simultaneous } \\
\text { Test }\end{array}$ \\
\hline Spec\#1 R & 7.7 & 6.0 & 14.5 & 16.2 \\
Spec\#2 L & 0.4 & 1.5 & 9.7 & 10.8 \\
Spec\#4 R & 2.2 & 4.7 & 14.6 & 13.4 \\
Spec\#14 R & 1.6 & 2.3 & 8.3 & 11.8 \\
Spec\#17 R & 8.8 & 4.2 & 4.6 & 6.1 \\
Spec\# 20 R & 8.0 & 6.1 & 10.9 & 7.7 \\
Sepc\#21 R & 4.5 & 5.3 & 11.9 & 9.2 \\
Mean & $\mathbf{4 . 7}$ & $\mathbf{4 . 3}$ & $\mathbf{1 0 . 6}$ & $\mathbf{1 0 . 7}$ \\
Std & $\mathbf{3 . 4}$ & $\mathbf{1 . 8}$ & $\mathbf{3 . 5}$ & $\mathbf{3 . 4}$ \\
& & & & \\
\hline & Internal & Internal & External & External \\
Harvested & Rotation & Rotation & Rotation & Rotation \\
(Plate+ Screw) & Sequential Test & Simultaneous & Sequential Test & Simultaneous \\
& 9.2 & Test & 11.7 & Test \\
\hline Spec\#3 L & 4.8 & 6.2 & 7.9 \\
Spec\#5 L & 4.8 & 2.8 & 12.0 & 7.9 \\
Spec\#6 L & 0.9 & 0.8 & 11.9 & 10.9 \\
Spec\#9 L & 10.5 & 9.2 & 6.1 & 13.5 \\
Spec\#13 R & 0.9 & 1.8 & $\mathbf{8 . 5}$ & 4.8 \\
Spec\#15 L & 0.3 & 2.9 & $\mathbf{3 . 9}$ & $\mathbf{8 . 2}$ \\
Mean & $\mathbf{4 . 4}$ & $\mathbf{4 . 4}$ & $\mathbf{3 . 6}$ \\
Std & $\mathbf{4 . 5}$ & & & \\
\hline
\end{tabular}


Table 22: Relative rotation about $Y^{\prime}$ axis (instrumented state) in joint frame

\begin{tabular}{|c|c|c|c|c|}
\hline Screws & $\begin{array}{c}\text { Internal } \\
\text { Rotation } \\
\text { Sequential Test }\end{array}$ & $\begin{array}{c}\text { Internal } \\
\text { Rotation } \\
\text { Simultaneous } \\
\text { Test } \\
\end{array}$ & $\begin{array}{c}\text { External } \\
\text { Rotation } \\
\text { Sequential Test }\end{array}$ & $\begin{array}{c}\text { External } \\
\text { Rotation } \\
\text { Simultaneous } \\
\text { Test } \\
\end{array}$ \\
\hline Spec\#1 R & 4.8 & 6.3 & 7.3 & 5.9 \\
\hline Spec\#2 L & 1.9 & 0.9 & 6.0 & 6.8 \\
\hline Spec\#4 R & 3.1 & 1.3 & 4.7 & 6.9 \\
\hline Spec\#14 R & 1.6 & 0.1 & 3.4 & 2.6 \\
\hline Spec\#17 R & 1.5 & 5.1 & 0.9 & 1.7 \\
\hline Spec\# 20 R & 1.1 & 1.5 & 6.2 & 8.0 \\
\hline Sepc\#21 R & 6.5 & 3.4 & 6.8 & 5.6 \\
\hline Mean & 2.9 & 2.6 & 5.0 & 5.4 \\
\hline Std & 2.0 & 2.3 & 2.2 & 2.3 \\
\hline Plate + Screw & $\begin{array}{c}\text { Internal } \\
\text { Rotation } \\
\text { Sequential Test }\end{array}$ & $\begin{array}{c}\text { Internal } \\
\text { Rotation } \\
\text { Simultaneous } \\
\text { Test } \\
\end{array}$ & $\begin{array}{c}\text { External } \\
\text { Rotation } \\
\text { Sequential Test }\end{array}$ & $\begin{array}{c}\text { External } \\
\text { Rotation } \\
\text { Simultaneous } \\
\text { Test } \\
\end{array}$ \\
\hline Spec\#3 L & 4.1 & 4.6 & 2.0 & 3.3 \\
\hline Spec\#5 L & 0.2 & 2.4 & 5.0 & 5.3 \\
\hline Spec\#6 L & 2.8 & 0.4 & 4.3 & 3.6 \\
\hline Spec\#9 L & 2.2 & 3.7 & 0.6 & 0.1 \\
\hline Spec\#13 R & 2.5 & 3.1 & 0.4 & 3.2 \\
\hline Spec\#15 L & 0.5 & 1.2 & 5.2 & 5.9 \\
\hline Mean & 2.1 & 2.6 & 2.9 & 3.6 \\
\hline Std & 1.5 & 1.6 & 2.2 & 2.0 \\
\hline
\end{tabular}


Table 23: Relative rotation about $Z^{\prime}$ axis (harvested/native state) in joint frame

\begin{tabular}{lcccc}
\hline $\begin{array}{c}\text { Harvested } \\
\text { (Screws) }\end{array}$ & $\begin{array}{c}\text { Internal } \\
\text { Rotation } \\
\text { Sequential Test }\end{array}$ & $\begin{array}{c}\text { Internal } \\
\text { Rotation } \\
\text { Simultaneous } \\
\text { Test }\end{array}$ & $\begin{array}{c}\text { External } \\
\text { Rotation } \\
\text { Sequential Test }\end{array}$ & $\begin{array}{c}\text { External } \\
\text { Rotation } \\
\text { Simultaneous } \\
\text { Test }\end{array}$ \\
\hline Spec\#1 R & 0.3 & 0.9 & 6.1 & 5.9 \\
Spec\#2 L & 1.8 & 2.2 & 2.2 & 1.2 \\
Spec\#4 R & 1.5 & 2.3 & 4.8 & 5.2 \\
Spec\#14 R & 0.3 & 0.1 & 3.6 & 2.3 \\
Spec\#17 R & 1.6 & 0.1 & 12.5 & 12.9 \\
Spec\# 20 R & 0.5 & 0.8 & 5.9 & 6.0 \\
Sepc\#21 R & 0.3 & 0.0 & 3.7 & 4.1 \\
Mean & $\mathbf{0 . 9}$ & $\mathbf{0 . 9}$ & $\mathbf{5 . 5}$ & $\mathbf{5 . 4}$ \\
Std & $\mathbf{0 . 7}$ & $\mathbf{1 . 0}$ & $\mathbf{3 . 4}$ & $\mathbf{3 . 8}$ \\
& & & & External \\
Harvested & Internal & Internal & External & Rotation \\
(Plate + Screw) & Sequential Test & Simultaneous & Rotation & Simultaneous \\
& Tequential Test & Test \\
\hline Spec\#3 L & 0.8 & 2.2 & 0.3 & 2.0 \\
Spec\#5 L & 1.9 & 0.4 & 4.4 & 4.3 \\
Spec\#6 L & 2.2 & 3.5 & 2.6 & 3.2 \\
Spec\#9 L & 2.1 & 2.4 & 3.7 & 5.7 \\
Spec\#13 R & 0.1 & 0.4 & 4.7 & 4.5 \\
Spec\#15 L & 0.1 & 0.9 & 3.2 & 3.4 \\
Mean & $\mathbf{1 . 2}$ & $\mathbf{1 . 6}$ & $\mathbf{3 . 2}$ & $\mathbf{1 . 3}$ \\
Std & $\mathbf{1 . 0}$ & $\mathbf{1 . 2}$ & $\mathbf{1 . 6}$ & \\
\hline
\end{tabular}


Table 24: Relative rotation about $Z^{\prime}$ axis (instrumented state) in joint frame

\begin{tabular}{lcccc}
\hline \multicolumn{1}{c}{ Screws } & $\begin{array}{c}\text { Internal } \\
\text { Rotation } \\
\text { Sequential Test }\end{array}$ & $\begin{array}{c}\text { Internal } \\
\text { Rotation } \\
\text { Simultaneous } \\
\text { Test }\end{array}$ & $\begin{array}{c}\text { External } \\
\text { Rotation } \\
\text { Sequential Test }\end{array}$ & $\begin{array}{c}\text { External } \\
\text { Rotation } \\
\text { Simultaneous } \\
\text { Test }\end{array}$ \\
\hline Spec\#1 R & 1.1 & 0.1 & 4.6 & 4.6 \\
Spec\#2 L & 1.9 & 1.6 & 2.2 & 2.6 \\
Spec\#4 R & 2.2 & 2.4 & 1.2 & 2.2 \\
Spec\#14 R & 1.4 & 1.4 & 0.8 & 0.6 \\
Spec\#17 R & 0.6 & 2.5 & 2.3 & 4.5 \\
Spec\# 20 R & 0.9 & 1.2 & 1.9 & 2.1 \\
Sepc\#21 R & 0.9 & 1.2 & 4.8 & 4.5 \\
Mean & $\mathbf{1 . 3}$ & $\mathbf{1 . 5}$ & $\mathbf{2 . 5}$ & $\mathbf{3 . 0}$ \\
Std & $\mathbf{0 . 6}$ & $\mathbf{0 . 8}$ & $\mathbf{1 . 6}$ & $\mathbf{1 . 5}$ \\
& & & & \\
\hline & Internal & Internal & External & External \\
Plate + Screw & Rotation & Rotation & Rotation & Rotation \\
& Sequential Test & Simultaneous & Sequential Test & Simultaneous \\
\hline Spec\#3 L & 1.1 & Test & 0.5 & Test \\
Spec\#5 L & 1.1 & 1.2 & 0.7 & 1.8 \\
Spec\#6 L & 0.1 & 0.4 & 0.4 & 1.2 \\
Spec\#9 L & 0.4 & 0.6 & 1.2 & 0.0 \\
Spec\#13 R & 2.9 & 2.9 & 0.0 & 0.8 \\
Spec\#15 L & 0.5 & 0.4 & 1.1 & 1.0 \\
Mean & $\mathbf{1 . 0}$ & $\mathbf{1 . 0}$ & $\mathbf{0 . 6}$ & 1.2 \\
Std & $\mathbf{1 . 0}$ & $\mathbf{1 . 0}$ & $\mathbf{0 . 4}$ & $\mathbf{1 . 0}$ \\
& & & & $\mathbf{0 . 6}$ \\
\hline
\end{tabular}




\section{APPENDIX H: DEMOGRAPHIC DATA}

Table 25: Demographic details of specimens

\begin{tabular}{lllllll}
\hline $\begin{array}{l}\text { Pair } \\
\text { number }\end{array}$ & $\begin{array}{l}\text { Specimen } \\
\text { number }\end{array}$ & Side & Instrumentation & $\begin{array}{l}\text { Original } \\
\text { specimen } \\
\text { number }\end{array}$ & Age & Sex \\
\hline 1 & 15 & Left & Plate + screw & RLU0202103 & 52 & Female \\
2 & 14 & Right & Two screws & RLU0202104 & 52 & Female \\
& 5 & Left & Plate + screw & RLU1118091 & 75 & Female \\
3 & 21 & Right & Two screws & RLU1118092 & 75 & Female \\
& 2 & Left & Two screws & RLU1115091 & 73 & Female \\
4 & 13 & Right & Plate + screw & RLU1115092 & 73 & Female \\
& 3 & Left & Plate + screw & RLU1125091 & 54 & Male \\
5 & 1 & Right & Two screws & RLU1125092 & 54 & Male \\
& 6 & Left & Plate + screw & RLU1125093 & 75 & Female \\
6 & 17 & Right & Two screws & RLU1125094 & 75 & Female \\
& 9 & Left & Plate + screw & RLU0119101 & 56 & Female \\
7 & 20 & Right & Two screws & RLU0119102 & 56 & Female \\
\hline
\end{tabular}

Age range: 52 to 75 years

Mean age: 65 years

Median age: 73 years

No of females: 10 out of 13 specimens

Percentage of females: $71.43 \%$ 


\section{VITA}

Rahul Sudheer Ghotge was born in 1981 in Pune, India. Having completed initial education at Muktangan English School, Pune and Sir Parshurambhau College, Pune, he began his medical education at Byramjee Jeejeebhoy Medical College, Pune in 1999. He successfully completed final MBBS examination (equivalent to doctor of medicine in United States of America) in December 2003. Following this, he joined Sassoon General Hospitals, Pune in 2004 and later went to United Kingdom in 2005 to begin his training to become Member of Royal College of Surgeons. In May 2006, he returned to India and worked in the field of orthopedics. While working in orthopedics, he began to identify numerous shortcomings in the currently available orthopedic devices. Therefore in August of 2008, he joined the Master of Science program in Biomedical Engineering and Imaging at the University of Tennessee Health Science Center, Memphis with an intention to study human biomechanics and orthopedic implants. While at University of Tennessee, he worked as Graduate Research Assistant at the biomechanics laboratory under the guidance of Denis DiAngelo, Ph.D. He worked on adaptation of a multi-axis programmable test frame, popularly known as the spine robot, to study biomechanics in the human cadaveric foot. He created a human cadaveric foot model and used it to study the biomechanics of the talonavicular joint. In 2010, he completed his Master's degree. 